\title{
WestVirginiaUniversity
}

THE RESEARCH REPOSITORY @ WVU

Graduate Theses, Dissertations, and Problem Reports

2018

\section{Pulsars as Calibration Tools and X-Ray Observations of Spider Pulsars}

\author{
Peter Anthony Gentile
}

Follow this and additional works at: https://researchrepository.wvu.edu/etd

\section{Recommended Citation}

Gentile, Peter Anthony, "Pulsars as Calibration Tools and X-Ray Observations of Spider Pulsars" (2018). Graduate Theses, Dissertations, and Problem Reports. 5661.

https://researchrepository.wvu.edu/etd/5661

This Dissertation is protected by copyright and/or related rights. It has been brought to you by the The Research Repository @ WVU with permission from the rights-holder(s). You are free to use this Dissertation in any way that is permitted by the copyright and related rights legislation that applies to your use. For other uses you must obtain permission from the rights-holder(s) directly, unless additional rights are indicated by a Creative Commons license in the record and/ or on the work itself. This Dissertation has been accepted for inclusion in WVU Graduate Theses, Dissertations, and Problem Reports collection by an authorized administrator of The Research Repository @ WVU.

For more information, please contact researchrepository@mail.wvu.edu. 


\title{
Pulsars as Calibration Tools and X-Ray Observations of Spider Pulsars
}

\author{
Peter Anthony Gentile \\ Dissertation Submitted to \\ The Eberly College of Arts and Sciences \\ at West Virginia University \\ in partial fulfillment of the requirements for the degree of \\ Doctor of Philosophy in \\ Physics \\ Maura McLaughlin, Ph.D., Chair \\ Duncan Lorimer, Ph.D. \\ Loren Anderson, Ph.D. \\ Paul Miller, Ph.D. \\ Zachariah Etienne, Ph.D. \\ Department of Physics and Astronomy \\ Morgantown, WV \\ 2018
}

Keywords: Pulsars; Neutron Stars; Radio Polarimetry

Copyright 2018 Peter Gentile 


\section{ABSTRACT \\ Pulsars as Calibration Tools \\ and X-Ray Observations of Spider Pulsars}

\section{Peter Anthony Gentile}

We present the polarization pulse profiles for 29 pulsars observed with the Arecibo Observatory by the North American Nanohertz Observatory for Gravitational Waves (NANOGrav) timing project at $2.1 \mathrm{GHz}, 1.4 \mathrm{GHz}$, and $430 \mathrm{MHz}$. These profiles represent the most sensitive polarimetric millisecond pulsar profiles to date, revealing the existence of microcomponents (that is, pulse components with peak intensities much lower than the total pulse peak intensity). Although microcomponents have been detected in some pulsars previously, we are able to detect new microcomponents for PSRs B1937+21, J1713+0747, and J2234+0944. We also present rotation measures for 28 of these pulsars, determined independently at different observation frequencies and epochs, and find the Galactic magnetic fields derived from these rotation measures to be consistent with current models. These polarization profiles were made using measurement equation template matching, which allows us to generate the polarimetric response of the Arecibo Observatory on an epoch-by-epoch basis. We use this method to describe its time variability, and find that the polarimetric responses of the Arecibo Observatorys 1.4 and 2.1 $\mathrm{GHz}$ receivers varies significantly with time.

We then describe the first X-ray observations of five short orbital period $\left(P_{B}<\right.$ 1 day), $\gamma$-ray emitting, binary millisecond pulsars. Four of these- PSRs J0023+0923, J1124-3653, J1810+1744, and J2256-1024- are "black-widow" pulsars, with degenerate companions of mass $\ll 0.1 M_{\odot}$, three of which exhibit radio eclipses. The fifth source, PSR J2215+5135, is an eclipsing "redback" with a near Roche-lobe filling $0.2 M_{\odot}$ non-degenerate companion. Data were taken using the Chandra $X$-Ray Observatory and covered a full binary orbit for each pulsar. Two pulsars, PSRs J2215+5135 and J2256-1024, show significant orbital variability while PSR J1124-3653 shows marginal orbital variability. The lightcurves for these three pulsars have X-ray flux minima coinciding with the phases of the radio eclipses. This phenomenon is consistent with an intrabinary shock emission interpretation for the X-rays. The other two pulsars, PSRs J0023+0923 and J1810+1744, are fainter and do not demonstrate variability at a level we can detect in these data. All five spectra are fit with three separate models: a power-law model, a blackbody model, and a combined model with both power-law and blackbody components. The preferred spectral fits yield power-law indices that range from 1.3 to 3.2 and blackbody temperatures in the hundreds of eV. The spectrum for PSR J2215+5135 shows a significant hard X-ray component, with a large number of counts above $2 \mathrm{keV}$, which 
is additional evidence for the presence of intrabinary shock emission. This is similar to what has been detected in the low-mass X-ray binary to MSP transition object PSR J1023+0038.

We also describe X-Ray observations of three "redback" pulsars taken with the $X M M-N e w t o n$ X-Ray telescope, and cover at least one orbit for each source. We had previously analyzed data for one of these sources, PSR J2215+5135, taken with the Chandra $X$-Ray Observatory. These new observations also show orbital variability in PSR J2215+5135's X-Ray lightcurve, including an X-Ray minimum near superior conjunction, and the increased sensitivity allows us to see two clear features away from superior conjunction. For the other two sources, PSRs J1622-0315 and J1908+2105, we do not detect enough counts to constrain the X-Ray orbital variability. The spectra for each of these sources showed significant hard X-Ray emission, and were therefore not well described by thermal models. We report power-law indices from these fits in the range of 1.28 to 2.0. These spectral properties are consistent with intrabinary shock emission. 


\section{Dedication}

To my father, Joseph Paul Gentile Jr. 


\section{Acknowledgements}

My thesis, as I would presume is true of every thesis, should in all good conscience have an author list that is much longer than one person. As West Virginia University would surely frown on this, I hope this small expression of my gratitude will suffice.

Remembering everyone that should be thanked is going to be impossible, especially for me, but any list must start with Maura McLaughlin. I very simply would not have been able to do this without her help. Along with her indispensable help with research, she has also provided me with every opportunity to travel, and in doing so, meet new people, see new places, and of course spread the coolness of pulsar astronomy. I would also like to thank the members of my committee: Duncan Lorimer, Loren Anderson, Paul Miller, Amy Hessl, and especially Zachariah Etienne, who jumped in at the last minute without hesitation. Thanks also to Mallory Roberts, not only for his guiding hand in the world of X-Ray astronomy, but also for the way that conversations always seemed to devolve into a combination of research strategy, incredulity of other people's methods, and a fanciful love of X-Ray astronomy. I left every conversation a better researcher.

This endeavor would also not have been possible without the love and support of my family. Especially from my mother, Rose, who is an embodiment of the strength and determination needed for an undertaking such as this and has therefore been an inspiration to me. To my siblings, Mark and Julie, for being a huge source of support; to my nieces, Olivia and Josie, for serving the pivotal role of being the adults in the room whenever we would hang out; and to Bill, Ruth, Candice, and Jared, for being such an integral part of my family experience when I return home. In the interest of saving the poor trees upon which this would be printed, I will not list every member of my extended family, but I will say that each and every one of them deserves it for the unique contribution they all add to make me me.

I also had the enormous fortune of meeting and becoming friends with some truly incredible people in my time in Morgantown. To Joe Swiggum, Sarah Halls, Lucas Hunt, Johanna Hunt, Fernando Cardoso, and the ever-suave Mehran Mohebbi: I cannot imagine grad school without you all. To Maria Grazia Blasi for opening her home and family to me; to Dr. Brook and Uthpala Herath for being so lit; to Andrew Seymour for being the most interesting roommate I have ever had; to Josh Miller and Mitch Mickaliger for helping me keep my sanity in that dungeonous office; to Amy Sardone and Nick Pingel for taking my eerie silence in the office in stride; to Dr. Lam for being the only person who is more frustrated than me with file headers; to Golnoosh Golpayegani, Pariya Pourmohammadi, Faezeh Sedighi, and Pedram Tavadze for adding immeasurably to my life in Morgantown; to Alyse May and Alivia Margaret Bartifay for teaching me what bolus is; to Elisa Legno for introducing me to the world of ginsengs; to Veronica Ghezzi, Beatrice Malvisi, Annastella Vester and the rest of the Italian Studies department for dealing with my pestering questions; to everyone at the Elk's Lodge \#411; and to all of those who played such an important role in my formative undergrad years, including Bill Sheers, Zac Talmadge, Christian Miedel, Conor Demers, and Mike Graysay.

I cannot thank you all enough. 


\section{Table of Contents}

List of Tables $\quad x$

List of Figures $\quad$ xi

1 Introduction 1

1.1 The History of Pulsars . . . . . . . . . . . . . . . . . . . . . . . . 1

1.2 Emission Mechanisms . . . . . . . . . . . . . . . . . . . 3

1.2.1 The Pulsar Magnetosphere . . . . . . . . . . . . . . . 7

1.2.2 Acceleration Gaps . . . . . . . . . . . . . . . . . . . . . 14

1.2.2.1 Polar Gap . . . . . . . . . . . . . . . . . . . . . 14

1.2 .2 .2 Outer Gap . . . . . . . . . . . . . . . . . . . . . . . . . . 17

1.3 Pulsar Types . . . . . . . . . . . . . . . . . . . . . . . . . . 18

1.3.1 Canonical Pulsars . . . . . . . . . . . . . . . . 23

1.3.2 Millisecond Pulsars . . . . . . . . . . . . . . . . . . 24

1.3.3 Black Widow and Redback Pulsars . . . . . . . . . . . . . 26

1.4 Thesis Outline . . . . . . . . . . . . . . . . . . . . 30

2 Pulsar Polarimetry 33

2.1 Radio Polarimetry . . . . . . . . . . . . . . . . . 33

2.1.1 Toy Receiver and Stokes Parameters . . . . . . . . . . . . . 33

2.1.2 Mueller Matrices ... . . . . . . . . . . . . . . . . . 42

2.1.3 Ideal Feed Assumption . . . . . . . . . . . . . . . . . 49

2.1.4 Full Calibration . . . . . . . . . . . . . . . 52

2.2 Rotating Vector Model . . . . . . . . . . . . . . . . . . . . 53

2.3 Faraday Rotation . . . . . . . . . . . . . . 56

3 The NANOGrav Nine-Year Data Set and Beyond: Polarimetry and Pulse $\begin{array}{ll}\text { Microcomponents } & 61\end{array}$

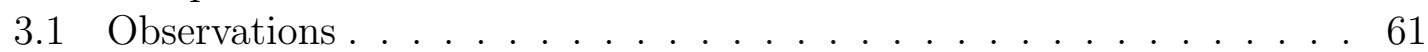

3.2 Data Reduction . . . . . . . . . . . . . . . . 62

3.2.1 Polarimetric Calibration . . . . . . . . . . . . . 62

3.2.1.1 Measurement Equation Modeling . . . . . . . . . . . 62

3.2.2 Faraday Rotation Correction ................ 65

3.3 Results . . . . . . . . . . . . . . . . . . 65

3.3.1 PSR J0023+0923 . . . . . . . . . . . . . . . . . 68

3.3.1.1 1.4 GHz .................. . . 68

3.3.1.2 $430 \mathrm{MHz}$................... . . 69

3.3.2 PSR J0030+0451 . . . . . . . . . . . . . . . . . . 70

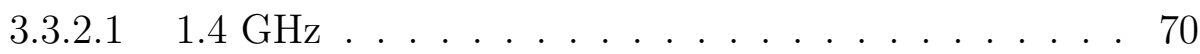

3.3.2.2 $430 \mathrm{MHz} \ldots \ldots . \ldots . \ldots 71$

3.3.3 PSR J1022+1001 . . . . . . . . . . . . . . . . . 71

3.3.3.1 $430 \mathrm{MHz}$.................... . . 71

3.3.4 PSR J1453+1902 . . . . . . . . . . . . . . . . . . . 72 
3.3.4.1 $2.1 \mathrm{GHz} \ldots \ldots \ldots \ldots . \ldots . \ldots 72$

3.3.4.2 1.4 GHz ..................... 72

3.3.4.3 $430 \mathrm{MHz}$. . . . . . . . . . . . . . . 73

3.3.5 PSR J1640+2224 . . . . . . . . . . . . . . . . . . 73

3.3.5.1 $1.4 \mathrm{GHz} \ldots \ldots \ldots \ldots . \ldots . \ldots . \ldots 73$

3.3.5.2 $430 \mathrm{MHz} \ldots \ldots \ldots \ldots . \ldots . \ldots 74$

3.3.6 PSR J1709+2313 . . . . . . . . . . . . . . . . . . . 74

3.3.6.1 1.4 GHz .................. . . . 74

3.3.7 PSR J1713+0747 . . . . . . . . . . . . . . . . . . 74

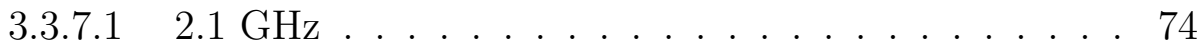

3.3.7.2 1.4 GHz .................. . . 75

3.3.8 PSR J1738+0333 . . . . . . . . . . . . . . . . . 76

3.3.8.1 2.1 GHz ................... 76

3.3.8.2 1.4 GHz . . . . . . . . . . . . . . 76

3.3 .9 PSR J1741+1351 . . . . . . . . . . . . . . . . . . . . . . . . . . . . . . . . . 77

3.3.9.1 2.1 GHz ................... . . 77

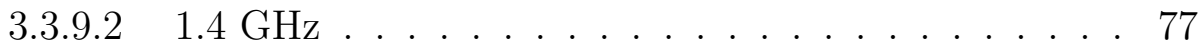

3.3.9.3 $430 \mathrm{MHz}$.................. . . 80

3.3 .10 PSR J1853+1303 . . . . . . . . . . . . . . . . . 80

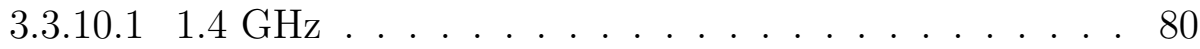

3.3.10.2 $430 \mathrm{MHz}$................ . . 81

3.3.11 PSR B1855+09 . . . . . . . . . . . . . . . . 81

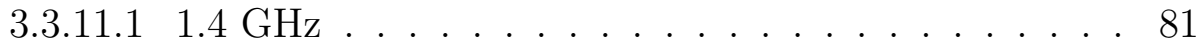

3.3.11.2 $430 \mathrm{MHz}$.................. . . . 82

3.3.12 PSR J1903+0327 . . . . . . . . . . . . . . . . . . . . . . . . . . . . . . . . . 83

3.3.12.1 2.1 GHz ................... . . 83

3.3.12.2 $1.4 \mathrm{GHz}$... . . . . . . . . . . . . . . 83

3.3.13 PSR J1910+1256 . . . . . . . . . . . . . . . . 84

3.3.13.1 2.1 GHz .................. . . . 84

3.3.13.2 1.4 GHz . . . . . . . . . . . . . . . . . 84

3.3.14 PSR J1911+1347 . . . . . . . . . . . . . . . . . . 85

3.3.14.1 2.1 GHz .................. . . 85

$3.3 .14 .21 .4 \mathrm{GHz} \ldots \ldots \ldots \ldots 6$

3.3.14.3 $430 \mathrm{MHz}$................. . . 86

3.3 .15 PSR J1923+2515 . . . . . . . . . . . . . . . . 86

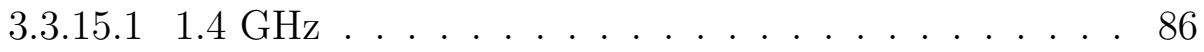

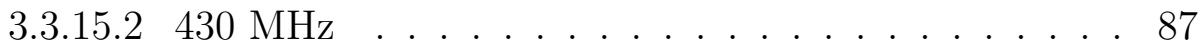

3.3.16 PSR B1937+21 . . . . . . . . . . . . . . . . . 88

$3.3 .16 .12 .1 \mathrm{GHz} \ldots \ldots \ldots \ldots$

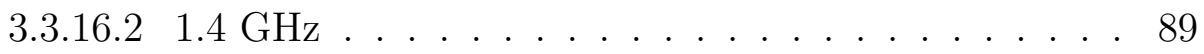

3.3.17 PSR J1944+0907 . . . . . . . . . . . . . . . . . . . 90

3.3.17.1 2.1 GHz . . . . . . . . . . . . . . . . 90

3.3.17.2 1.4 GHz .................. . . 90

3.3.17.3 $430 \mathrm{MHz}$. . . . . . . . . . . . . . . 91

3.3.18 PSR J1949+3106 . . . . . . . . . . . . . . . . . 92 
3.3.18.1 2.1 GHz . . . . . . . . . . . . . . . . 92

3.3.18.2 1.4 GHz ................... . . . . . . . . . . . . . . 92

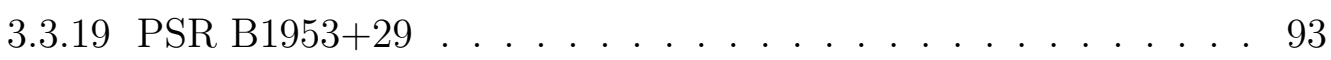

3.3.19.1 1.4 GHz . . . . . . . . . . . . . . . 93

3.3.20 PSR J1955+2527 . . . . . . . . . . . . . . . . . 93

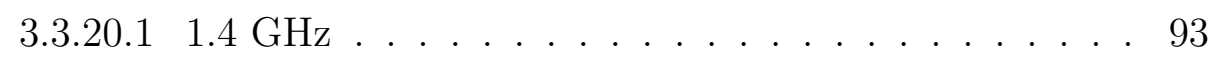

3.3.21 PSR J2017+0603 . . . . . . . . . . . . . . . . . . . . 94

3.3.21.1 2.1 GHz .................. . . . 94

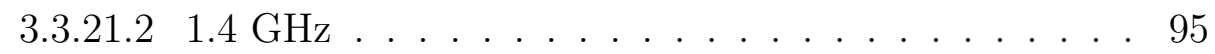

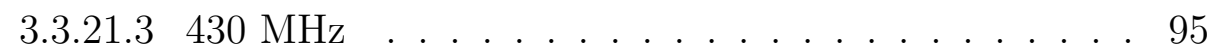

3.3 .22 PSR J2019+2425 . . . . . . . . . . . . . . . . 96

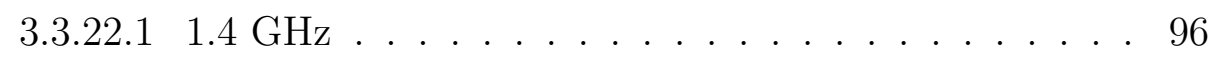

3.3 .23 PSR J2033+1734 . . . . . . . . . . . . . . . . . . . . . . . . . . . . . . . . . . . . 96

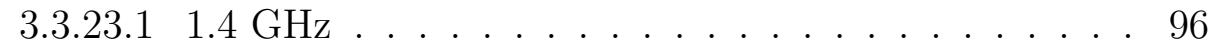

3.3.23.2 $430 \mathrm{MHz}$. . . . . . . . . . . . . . . . 97

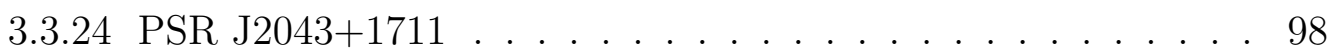

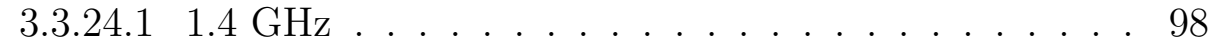

3.3.24.2 $430 \mathrm{MHz}$.................... . . . 98

3.3 .25 PSR J2214+3000 . . . . . . . . . . . . . . . . . . . . 99

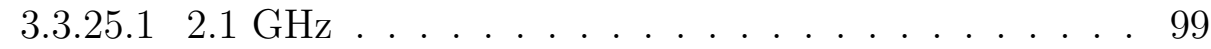

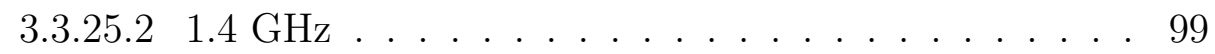

3.3 .26 PSR J2229+2643 . . . . . . . . . . . . . . . . . . . . . . . . . . . . . . . . . . . . . . . . . . .

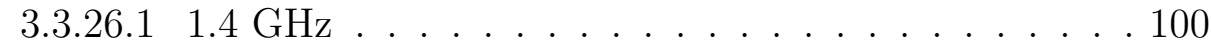

3.3.26.2 $430 \mathrm{MHz}$.................... . . . 101

3.3 .27 PSR J2234+0611 . . . . . . . . . . . . . . . . . 101

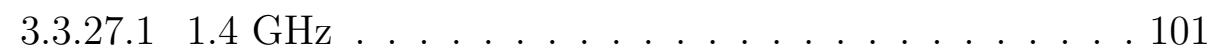

3.3 .28 PSR J2234+0944 . . . . . . . . . . . . . . . . . . . 102

3.3.28.1 2.1 GHz ........................ 102

3.3.28.2 $1.4 \mathrm{GHz}$. . . . . . . . . . . . . . . . 102

3.3.28.3 $430 \mathrm{MHz}$. . . . . . . . . . . . . . . 103

3.3 .29 PSR J2317+1439 . . . . . . . . . . . . . . . . . 103

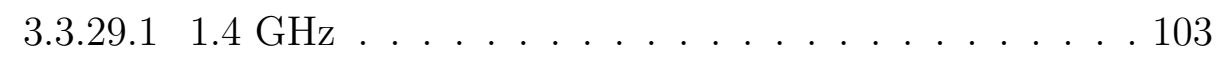

3.3.29.2 $430 \mathrm{MHz}$. . . . . . . . . . . . . . . 104

3.3.30 Resulting Polarimetric Responses . . . . . . . . . . . . . 105

3.4 Discussion . . . . . . . . . . . . . . . . . 107

4 X-Ray Observations of Black Widow Pulsars 113

4.1 Introduction . . . . . . . . . . . . . . . . . . . . . . 113

4.2 Observations and Analysis . . . . . . . . . . . . . . . . . . . . . . . . . . . . . . . . . . . .

4.3 Results . . . . . . . . . . . . . . . . . . 121

4.4 Discussion and Conclusions . . . . . . . . . . . . . . . 130 
$5 \quad$ X-Ray Observations of Redback Pulsars 136

5.1 Introduction . . . . . . . . . . . . . . . . . . 136

5.2 Observations and Analysis . . . . . . . . . . . . . . . . 139

5.3 Results . . . . . . . . . . . . . . . . . . 146

5.3 .1 PSR J1622-0315 . . . . . . . . . . . . . . . . . 146

5.3 .2 PSR J1908+2105 . . . . . . . . . . . . . . . . . 146

5.3 .3 PSR J2215+5135 . . . . . . . . . . . . . . . . . . . . . . . . 148

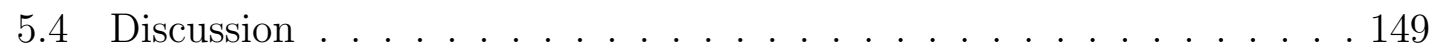

5 Conclusions 152

5.1 Pulsar Polarization and Receiver Modeling . . . . . . . . . . . . . 152

5.2 Spider Pulsars . . . . . . . . . . . . . . . . . . . 155

A Polarization Profiles of the NANOGrav Arecibo Dataset 159 


\section{List of Tables}

4.1 Timing and X-Ray Properties of Black Widow Sources . . . . . . . . 118

4.2 Chandra Spectral Fit Parameters . . . . . . . . . . . . . . . 119

5.1 Timing and X-ray Properties of Redback Sources . . . . . . . . . . . 144

$5.2 X M M-N e w t o n$ Spectral Fit Parameters . . . . . . . . . . . . . . 145

5.3 PSR J2215+5135 X-Ray Lightcurve Model Parameters . . . . . . . . 149 


\section{List of Figures}

1.1 Charge Distribution in the Pulsar Magnetosphere . . . . . . . . . . . 9

1.2 Pulsar Magnetosphere . . . . . . . . . . . . . . . . . . 11

1.3 Acceleration Gap locations . . . . . . . . . . . . . . . . . . . . . 13

1.4 High-energy Emission from the Crab Pulsar . . . . . . . . . . . . . . 19

$1.5 P-\dot{P}$ Diagram . . . . . . . . . . . . . . . . 21

2.1 Toy Receiver . . . . . . . . . . . . . . . . . . . . . . . 35

2.2 Poincaré Sphere . . . . . . . . . . . . . . . . . . . . . . . . . . . . . . . . . . . .

2.3 Stokes Parameters of a Noise Diode . . . . . . . . . . . . . . . 50

2.4 Stokes Parameters vs Parallactic Angle . . . . . . . . . . . . . . . 51

2.5 Pulsar Beam Geometry and Rotating Vector Model . . . . . . . . . . 54

3.1 Arecibo S-Wide Polarimetric Responses . . . . . . . . . . . . . . . . . 110

3.2 Arecibo L-Wide Polarimetric Responses . . . . . . . . . . . . . . . . . 111

3.3 RM-Derived Galactic Magnetic Field Strengths . . . . . . . . . . . . 112

4.1 Chandra X-Ray Lightcurve and Spectrum of PSR J0023+0923 . . . . 122

4.2 Chandra X-Ray Lightcurve and Spectrum of PSR J1124-3653 . . . . 124

4.3 Chandra X-Ray Lightcurve and Spectrum of PSR J1810+1744 . . . . 126

4.4 Chandra X-Ray Lightcurve and Spectrum of PSR J2215+5135 . . . . 127

4.5 Chandra X-Ray Lightcurve and Spectrum of PSR J2256-1024 . . . . 129

4.6 MSP X-Ray vs Spin-Down Luminosities . . . . . . . . . . . . . . 131

5.1 XMM-Newton Lightcurve and Spectrum of PSR J1622-0315 . . . . . 141

5.2 XMM-Newton Lightcurve and Spectrum of PSR J1908+2105 . . . . . 143

5.3 XMM-Newton Lightcurve and Spectrum of PSR J2215+5135 . . . . . 147

5.4 Spatial View of PSR J2215+5135's X-ray Lightcurve . . . . . . . . . 151

A.1 Polarization profile for PSR J0023+0923 at $1.4 \mathrm{GHz}$. . . . . . . . . . 160

A.2 Polarization profile for PSR J0023+0923 at $430 \mathrm{MHz}$. . . . . . . . . 161

A.3 Polarization profile for PSR J0030+0451 at $1.4 \mathrm{GHz}$. . . . . . . . . . 162

A.4 Polarization profile for PSR J0030+0451 at $430 \mathrm{MHz}$. . . . . . . . . 163

A.5 Polarization profile for PSR J1022+1001 at $430 \mathrm{MHz}$. . . . . . . . . 164

A.6 Polarization profile for PSR J1453+1902 at $2.1 \mathrm{GHz}$. . . . . . . . . . 165

A.7 Polarization profile for PSR J1453+1902 at $1.4 \mathrm{GHz}$. . . . . . . . . . 166

A.8 Polarization profile for PSR J1453+1902 at $430 \mathrm{MHz}$. . . . . . . . . 167

A.9 Polarization profile for PSR J1640+2224 at $1.4 \mathrm{GHz}$. . . . . . . . . . 168

A.10 Polarization profile for PSR J1640+2224 at $430 \mathrm{MHz}$. . . . . . . . . 169

A.11 Polarization profile for PSR J1709+2313 at $1.4 \mathrm{GHz}$. . . . . . . . . . 170

A.12 Polarization profile for PSR J1713+0747 at $2.1 \mathrm{GHz}$. . . . . . . . . . 171

A.13 Polarization profile for PSR J1713+0747 at $1.4 \mathrm{GHz}$. . . . . . . . . . 172

A.14 Polarization profile for PSR J1738+0333 at $2.1 \mathrm{GHz}$. . . . . . . . . . 173

A.15 Polarization profile for PSR J1738+0333 at $1.4 \mathrm{GHz}$. . . . . . . . . . 174

A.16 Polarization profile for PSR J1741+1351 at $2.1 \mathrm{GHz}$. . . . . . . . . . 175 
A.17 Polarization profile for PSR J1741+1351 at $1.4 \mathrm{GHz}$. . . . . . . . . . 176

A.18 Polarization profile for PSR J1741+1351 at $430 \mathrm{MHz}$. . . . . . . . . 177

A.19 Polarization profile for PSR J1853+1303 at $1.4 \mathrm{GHz}$. . . . . . . . . . 178

A.20 Polarization profile for PSR J1853+1303 at $430 \mathrm{MHz}$. . . . . . . . . 179

A.21 Polarization profile for PSR B1855+09 at $1.4 \mathrm{GHz}$. . . . . . . . . . . 180

A.22 Polarization profile for PSR B1855+09 at $430 \mathrm{MHz}$. . . . . . . . . . 181

A.23 Polarization profile for PSR J1903+0327 at $2.1 \mathrm{GHz}$. . . . . . . . . . 182

A.24 Polarization profile for PSR J1903+0327 at $1.4 \mathrm{GHz}$. . . . . . . . . . 183

A.25 Polarization profile for PSR J1910+1256 at $2.1 \mathrm{GHz}$. . . . . . . . . . 184

A.26 Polarization profile for PSR J1910+1256 at $1.4 \mathrm{GHz}$. . . . . . . . . . 185

A.27 Polarization profile for PSR J1911+1347 at $2.1 \mathrm{GHz}$. . . . . . . . . . 186

A.28 Polarization profile for PSR J1911+1347 at $1.4 \mathrm{GHz}$. . . . . . . . . . 187

A.29 Polarization profile for PSR J1911+1347 at $430 \mathrm{MHz}$. . . . . . . . . 188

A.30 Polarization profile for PSR J1923+2515 at $1.4 \mathrm{GHz}$. . . . . . . . . . 189

A.31 Polarization profile for PSR J1923+2515 at $430 \mathrm{MHz}$. . . . . . . . . 190

A.32 Polarization profile for PSR B1937+21 at $2.1 \mathrm{GHz}$. . . . . . . . . . . 191

A.33 Polarization profile for PSR B1937+21 at $1.4 \mathrm{GHz}$. . . . . . . . . . . 192

A.34 Polarization profile for PSR J1944+0907 at $2.1 \mathrm{GHz}$. . . . . . . . . . 193

A.35 Polarization profile for PSR J1944+0907 at $1.4 \mathrm{GHz}$. . . . . . . . . . 194

A.36 Polarization profile for PSR J1944+0907 at $430 \mathrm{MHz}$. . . . . . . . 195

A.37 Polarization profile for PSR J1949+3106 at $2.1 \mathrm{GHz}$. . . . . . . . . . 196

A.38 Polarization profile for PSR J1949+3106 at $1.4 \mathrm{GHz}$. . . . . . . . . . 197

A.39 Polarization profile for PSR B1953+29 at $1.4 \mathrm{GHz}$. . . . . . . . . . . 198

A.40 Polarization profile for PSR J1955+2527 at $1.4 \mathrm{GHz}$. . . . . . . . . . 199

A.41 Polarization profile for PSR J2017+0603 at $2.1 \mathrm{GHz}$. . . . . . . . . . 200

A.42 Polarization profile for PSR J2017+0603 at $1.4 \mathrm{GHz}$. . . . . . . . . . 201

A.43 Polarization profile for PSR J2017+0603 at $430 \mathrm{MHz}$. . . . . . . . . 202

A.44 Polarization profile for PSR J2019+2425 at $1.4 \mathrm{GHz}$. . . . . . . . . . 203

A.45 Polarization profile for PSR J2033+1734 at $1.4 \mathrm{GHz}$. . . . . . . . . . 204

A.46 Polarization profile for PSR J2033+1734 at $430 \mathrm{MHz}$. . . . . . . . . 205

A.47 Polarization profile for PSR J2043+1711 at $1.4 \mathrm{GHz}$. . . . . . . . . . 206

A.48 Polarization profile for PSR J2043+1711 at $430 \mathrm{MHz}$. . . . . . . . . 207

A.49 Polarization profile for PSR J2214+3000 at $2.1 \mathrm{GHz}$. . . . . . . . . . 208

A.50 Polarization profile for PSR J2214+3000 at $1.4 \mathrm{GHz}$. . . . . . . . . . 209

A.51 Polarization profile for PSR J2229+2643 at $1.4 \mathrm{GHz}$. . . . . . . . . . 210

A.52 Polarization profile for PSR J2229+2643 at $430 \mathrm{MHz}$. . . . . . . . . 211

A.53 Polarization profile for PSR J2234+0611 at $1.4 \mathrm{GHz}$. . . . . . . . . . 212

A.54 Polarization profile for PSR J2234+0944 at $2.1 \mathrm{GHz}$. . . . . . . . . . 213

A.55 Polarization profile for PSR J2234+0944 at $1.4 \mathrm{GHz}$. . . . . . . . . . 214

A.56 Polarization profile for PSR J2234+0944 at $430 \mathrm{MHz}$. . . . . . . . . 215

A.57 Polarization profile for PSR J2317+1439 at $1.4 \mathrm{GHz}$. . . . . . . . . . 216

A.58 Polarization profile for PSR J2317+1439 at $430 \mathrm{MHz}$. . . . . . . . . 217 


\section{Chapter 1}

\section{Introduction}

\subsection{The History of Pulsars}

In 1967, a graduate student named Jocelyn Bell was working at the University of Cambridge, studying how the solar wind affected the apparent brightness of bright, compact radio sources called quasars. Just as scintillation due to the atmospheric turbulence makes stars appear to twinkle to terrestrial stargazers, so does turbulent, ionized plasma make radio sources appear to fluctuate in intensity.

To study this phenomenon, she, along with her supervisor, Dr. Anthony Hewish, used an early radio telescope ${ }^{1}$ attached to a pen chart recorder to map the entire sky once every four days. She had no idea at the time, but she was helping to perform the very first pulsar survey.

Every day, this experiment generated 96 feet of pen chart data, and as this was analog data, they needed to be analyzed by hand. In keeping with tradition, this work was performed by the graduate student, which meant that Bell spent night after night poring over it. In that process, she discovered a signal she did not understand.

This in and of itself was not completely unexpected. Terrestrial radio frequency interference $(\mathrm{RFI})$ can be very insidious and is a fact of life for radio astronomers

\footnotetext{
${ }^{1}$ Just wires connected to posts in the ground, really.
} 
(past and present), so it was not really a surprise when Dr. Hewish put it forward as a possible explanation for the signal. Still, Bell kept seeing the source night after night, and it always appeared to be coming from the same position on the sky. This was particularly problematic to the RFI theory, since the time a given position in the sky is overhead changes day by day, which meant that either this was a source of RFI that was perfectly masquerading as an astronomical source, or, more simply, this was an astronomical source.

High time-resolution follow-up observations showed the source to be emitting 0.016 s-wide pulses at a period of 1.33 seconds. Observations of this source from another telescope ruled out the possibility of an instrumental effect causing the signal, and frequency-dependent delays of the pulses proved the source to be outside our solar system. Having convincingly determined the source to be real and astrophysical, the phenomenon was published in Hewish et al. (1968), however the origin of the emission proved to be elusive, and the strangeness of its regularity was compounded by the physical implication of the short duration of each pulse: light travel time constrains the emission of a 0.016 s-wide pulse to be coming from a source less than 4,800 km across. For a short time, the source was nicknamed LGM-1, "LGM" standing for "Little Green Men", as one possible theory was that the source may be an extraterrestrial beacon.

At this point, it is worth taking a step back. When considering the discovery story of pulsars, we often view it through the lens of hindsight, which tends to lend undue clarity to the story. We know what these signals are, so we may think of the name LGM-1 as a cute, perhaps silly, moniker for pulsars, thereby ascribing an 
attractive quaintness to the line of thought that led to it. A more illuminating vision of the name LGM-1, though, is as a barometer for the magnitude of the discovery. It was so momentous, so wonderfully unique, that the idea that it was the first evidence for intelligent, extraterrestrial life was not dismissed out of hand.

Thus, pulsar astronomy was born. Soon after, the Crab Pulsar was discovered (Staelin \& Reifenstein, 1968; Comella et al., 1969), discrediting the extraterrestrial beacon theory, and providing evidence that these objects were rotating beams of light emanating from dense stars called "neutron stars", thereby commencing the endeavor to explain the various characteristic of these pulsars and the environments they inhabit. We now turn to a small representation of the progress made in that endeavor. No current description of pulsars is complete, therefore what follows is an excitingly incomplete review of pulsar fundamentals as we understand them now.

\subsection{Emission Mechanisms}

A complete characterization of the pulsar magnetosphere (and thereby emission mechanism) has proven to be a notoriously difficult problem. As yet, no model exists to describe the rich nature of pulsar emission as seen from radio light to gamma ray light. Although there are characteristics it does not describe well, a simple model of the pulsar magnetosphere can provide explanations for a number of the characteristics of pulsar emission surprisingly well. This simple model, described by Goldreich \& Julian (1969), is that of a rotating dipolar magnetic field extending from a conducting sphere. 
Although even such a simple model includes much more complex characteristics, interesting deductions can be made from even from this simple foundation. For example, the model includes a time-varying magnetic field and will therefore produce electromagnetic radiation. This radiation will be emitted at the pulsar's spin frequency $(<1 \mathrm{kHz})$, and will therefore not be detectable through terrestrial observations, although it will produce other observable effects.

These effects stem from the fact that the production of electromagnetic radiation by the rotating dipole will serve to carry energy away from the system. The energy produced by a rotating dipole is

$$
\dot{E}_{\text {dipole }}=\frac{2}{3 c^{3}}|\mathbf{m}|^{2} \Omega^{4} \sin ^{2}(\alpha)
$$

where $c$ is the speed of light, $\mathbf{m}$ is the magnetic dipole moment, $\Omega$ is the rotational angular frequency, and $\alpha$ is the angle between the magnetic moment and spin axis. This energy is produced because the dipole's axis is offset from the spin axis. Therefore, the energy carried away by the radiation will be taken from the pulsar's rotational kinetic energy. Thus, the change in the pulsar's rotational kinetic energy

$$
\dot{E} \equiv-\frac{d E_{r o t}}{d t}=-\frac{d\left(I \Omega^{2} / 2\right)}{d t}=-I \Omega \dot{\Omega}
$$

where $I$ is the moment of inertia of the pulsar. Since the rotational angular frequency is related to the pulsar spin period $\mathrm{P}$ by

$$
\Omega=\frac{2 \pi}{P}
$$


the spin down luminosity is

$$
E_{\text {rot }}=4 \pi^{2} I \dot{P} P^{-3}
$$

Assuming the loss of rotational kinetic energy is due entirely to the magnetic dipole radiation, we can write

$$
\begin{gathered}
\dot{E}_{\text {dipole }}=\dot{E}_{\text {rot }} \\
\frac{8 \pi^{2}}{3 c^{3}}|\mathbf{m}|^{2} P^{-4} \sin ^{2}(\alpha)=I \dot{P} P^{-3} .
\end{gathered}
$$

Therefore,

$$
\frac{\dot{P}}{P}=\frac{8 \pi^{2}}{3 c^{3} I}|\mathbf{m}|^{2} \sin ^{2}(\alpha)
$$

Taking all terms other than $P$ and $\dot{P}$ and grouping them together in a constant $C$, we can write equation 1.6 as

$$
\begin{gathered}
P \dot{P}=C \\
P d P=C d t .
\end{gathered}
$$

Integrating both sides gives

$$
\frac{1}{2}\left(P^{2}-P_{0}^{2}\right)=C\left(t_{\text {now }}-t_{0}\right)
$$




$$
\frac{P^{2}}{2}\left(1-\left(\frac{P_{0}}{P}\right)^{2}\right)=C \tau_{c}=P \dot{P} \tau_{c}
$$

where $P_{0}$ is the birth period of the pulsar, $t_{\text {now }}$ is the current epoch, $t_{0}$ is the epoch of the pulsar's birth, and $\tau_{c}$ is the characteristic age of the pulsar (that is, the age of the pulsar given this simple model). Finally, since we know the pulsar will be spinning down throughout its life, which serves to increase the pulsar's spin period, we can ignore the $\left(\frac{P_{0}}{P}\right)^{2}$ term $^{2}$ and solve for $\tau_{c}$ to get

$$
\tau_{c}=\frac{P}{2 \dot{P}}
$$

Further, if we note that $|\mathbf{m}| \approx B r^{3}$, then we can rewrite equation 1.1 as

$$
\begin{gathered}
\frac{8 \pi^{2}}{3 c^{3}} B^{2} r^{6} P^{-4} \sin ^{2}(\alpha)=I \dot{P} P^{-3} \\
B^{2}=\frac{3 c^{3} I \sin ^{2}(\alpha)}{8 \pi^{2} r^{6}} \dot{P} P \\
B_{S}=D \sqrt{\dot{P} P}
\end{gathered}
$$

where, taking $\sin (\alpha)=1, r=10 \mathrm{~km}$, and $I=10^{45} \mathrm{~g} \mathrm{~cm}^{2}$, we find:

$$
B_{S}=3.2 \times 10^{19} \sqrt{\dot{P} P} \mathrm{G}
$$

Taking a step back, we have already gained an incredible amount of insight into pulsars from a simple model. Even with such a model, we are able to grasp

\footnotetext{
${ }^{2}$ Note that this is not true in general, as exceptions exist (see, for example, the sources discussed in Section 1.3.2). However, this exercise is not meant to be a rigorous determination of a pulsar's age, but rather rather a way to get a rough estimate, so we therefore proceed knowing that the utility of this estimate may vary from pulsar to pulsar.
} 
the pulsar's spin down behavior, and take guesses as to the pulsar's age and surface magnetic field strength. Still, since we are unable to see this dipolar radiation, we have not yet touched upon one of the most fundamental and enigmatic questions surrounding pulsars: what is producing the light that we detect with our telescopes? To begin talking about that, we must add a layer of complexity to our model.

\subsubsection{The Pulsar Magnetosphere}

One aspect of the model posited at the beginning of this section that we have yet to discuss is the superconductivity of the pulsar itself. This property is important because moving electromagnetic fields will induce additional fields, and this will have an effect on the charges within the pulsar.

Inside the neutron star, the rotation of the magnetic field will create an induced electric field

$$
\mathbf{E}_{\text {ind }}=(\boldsymbol{\Omega} \times \mathbf{r}) \times \mathbf{B}
$$

This surface charge density will induce a potential outside the neutron star of

$$
\Phi=\frac{B_{S} \Omega R^{5}}{6 c r^{3}}\left(3 \cos ^{2} \theta-1\right)
$$

where $B_{S}$ is the magnitude of the magnetic field at the surface of the neutron star, $R$ is the radius of the neutron star, and $r$ and $\theta$ are polar coordinates centered on the neutron star's center. We then find the component of the electric field at the surface of the neutron star parallel to the magnetic field to be 


$$
E_{\|}=\mathbf{E} \cdot \hat{B}=\left.\frac{\mathbf{E} \cdot \mathbf{B}}{B}\right|_{r=R}=-\frac{B_{S} \Omega R}{c} \cos ^{3} \theta .
$$

This electric field induces an electric force that is enormous and far exceeds the gravitational force acting to bind the charged particles to the neutron star. These charges then flow out from the neutron star and into the magnetosphere and continue to do so until the electric field due to the magentospheric charge density negates the electric field responsible for filling the magnetosphere with charged particles, making the magnetosphere a force-free region. The resulting charge distribution is

$$
\rho_{\text {mag }, f f}=-\frac{B_{S} \Omega R^{3}}{4 \pi c r^{3}}\left(3 \cos ^{2} \theta-1\right) \mathrm{C} .
$$

This charge distribution is pictured in Figure 1.1, from which (or alternatively from Equation 1.15) a number of characteristics may be gleaned. First, we see that the highest charge density occurs at the poles, at the surface of the neutron star. The number density here is called the "Goldreich-Julian" density, and from Equation 1.15 we can see that it is

$$
n_{G J}=-\frac{B_{S} \Omega}{2 \pi c e} .
$$

Conversely, we also note that there are regions of the magnetosphere that have a charge density of zero. These regions occur when $\left(3 \cos ^{2} \theta-1\right)=0$. That is, when $\theta=\cos ^{-1}(1 / \sqrt{3})$

The charged particles that fill the magnetosphere will stream out from the 


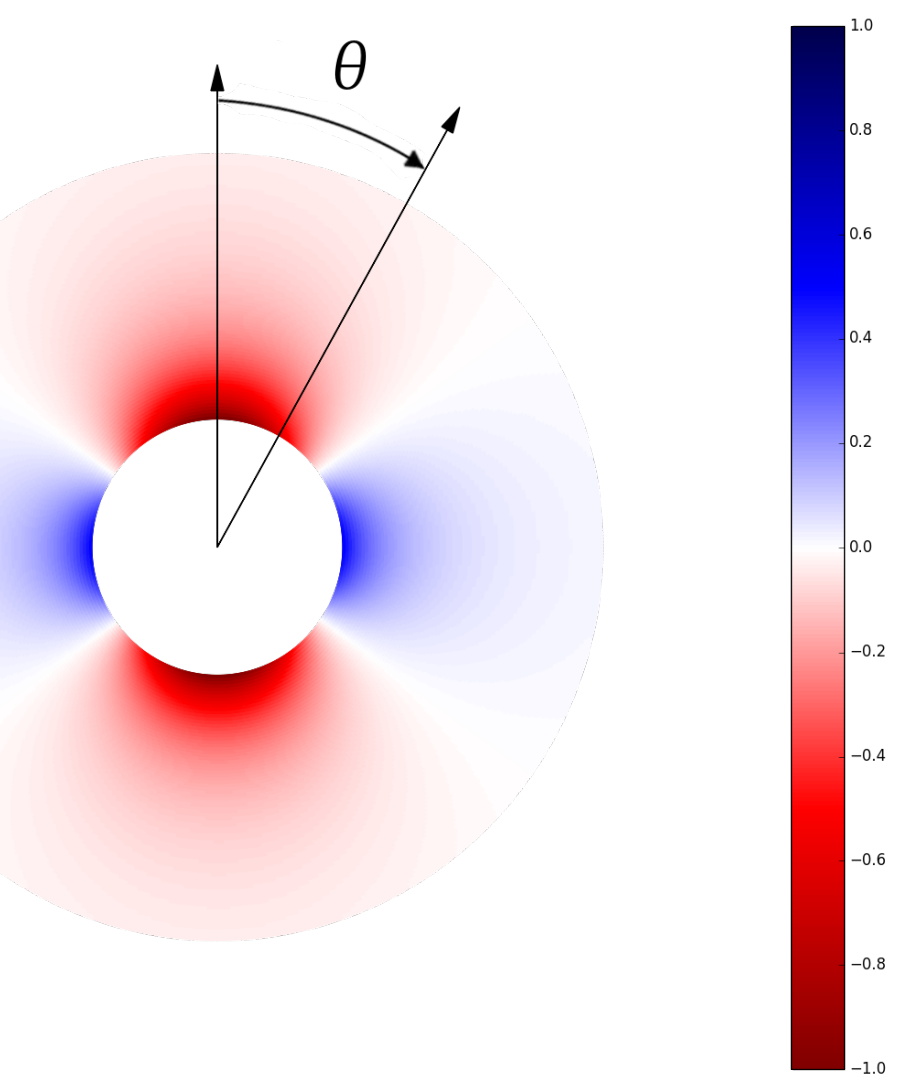

Figure 1.1: The normalized charge distribution in the pulsar magnetosphere, as given by Equation 1.15. Indicated angle represents the angle with respect to the aligned spin and magnetic field axes. Note the existence of the "null line" at $\theta=$ $\cos ^{-1}(1 / \sqrt{3})$, where the charge density drops to 0 . 
surface of the neutron star along its magnetic field lines. As there is a component of the electric field perpendicular to the magnetic field, these charged particles will gain a drift velocity

$$
v_{d}=\frac{\mathbf{E} \times \mathbf{B}}{B^{2}}
$$

which causes them to co-rotate with the neutron star. We note, however, that charged particles co-rotating above the neutron star can only maintain that corotation up to a certain distance from the neutron star. The surface beyond which co-rotation would require superluminal velocities is called the light cylinder, the radius of which depends only on the neutron star's spin period $P$ :

$$
R_{L C}=\frac{c P}{2 \pi}
$$

Note that for the fastest-spinning pulsars, this can be $<100 \mathrm{~km}$ !

Clearly, then, magnetic field lines (and charged particles moving along them) that do not cross the light cylinder can maintain co-rotation whereas magnetic field lines that do cross the light cylinder cannot. Therefore, this allows us to describe the neutron star's magnetosphere as being comprised of regions where the magnetic field lines do not cross the light cylinder and regions where the magnetic field lines do cross the light cylinder. The former are called closed field line regions while the latter are called open field line regions. As is shown in Figure 1.2, the open field line regions are centered on the two magnetic poles while the closed field line region resides above the magnetic equator. The eventual fate of the charged particles leaving the 


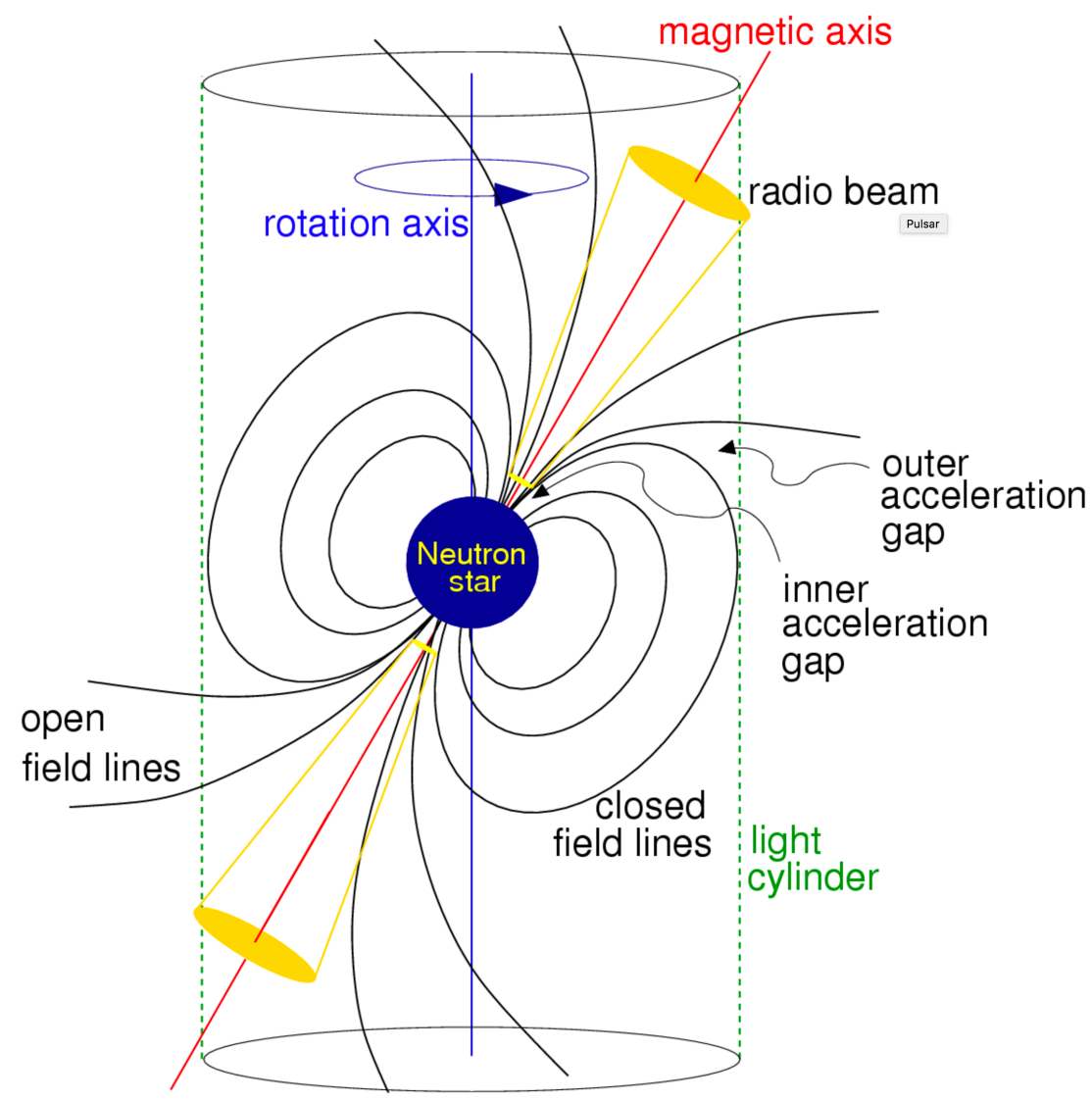

Figure 1.2: Model of a pulsar magnetosphere. A dipole magnetic field centered on the magnetic axis (shown in red) is offset from the neutron star spin axis (shown in blue). Shown in green is the light cylinder, or the surface past which co-rotation with the pulsar requires superluminal velocities and therefore cannot be maintained. Field lines which lie wholly inside this surface are called "closed field lines", while field lines which cross this surface are called "closed field lines". The closed field line region defines the extent of the acceleration gaps which produce most of the observed emission from the pulsar. (Image courtesy of Lorimer \& Kramer, 2005) 
neutron star surface depends greatly upon which region of the magnetosphere they enter upon leaving the neutron star surface.

Charges that enter the closed field line region flow from the neutron star and fill the magnetosphere until the resulting charge density reaches the density required for the region to become a force-free region (shown in Figure 1.1). Once this is achieved, the charges are only subject to the drift velocity that allows them to co-rotate with the neutron star (given in Equation 1.17).

Charged particles that enter open field line regions, however, do not undergo as simple a process as their closed field line region counterparts. As with those charged particles, the charged particles that enter open field line regions flow from the neutron star along magnetic field lines into the magnetosphere, and as before, they co-rotate with the neutron star. Eventually, though, these charged particles reach the light cylinder, are unable to maintain co-rotation, and leave the magnetosphere.

The fact that these charged particles can flow out from the magnetosphere means that the charge density in this part of the magnetosphere drops below the charge density required to maintain a force-free state (see Equation 1.17). The charged particles in this region, therefore, are subject not only to the drift velocity allowing them to co-rotate with the neutron star, but also to the force caused by the component of the electric field parallel to the magnetic field given in Equation 1.14. These regions of depleted charge density are areas wherein charged particles are accelerated, either from the neutron star surface into the plasma that makes up the bulk of the magnetosphere in the open field line region, or along the boundary of the open field line region and out of the magnetosphere though the light cylinder. 


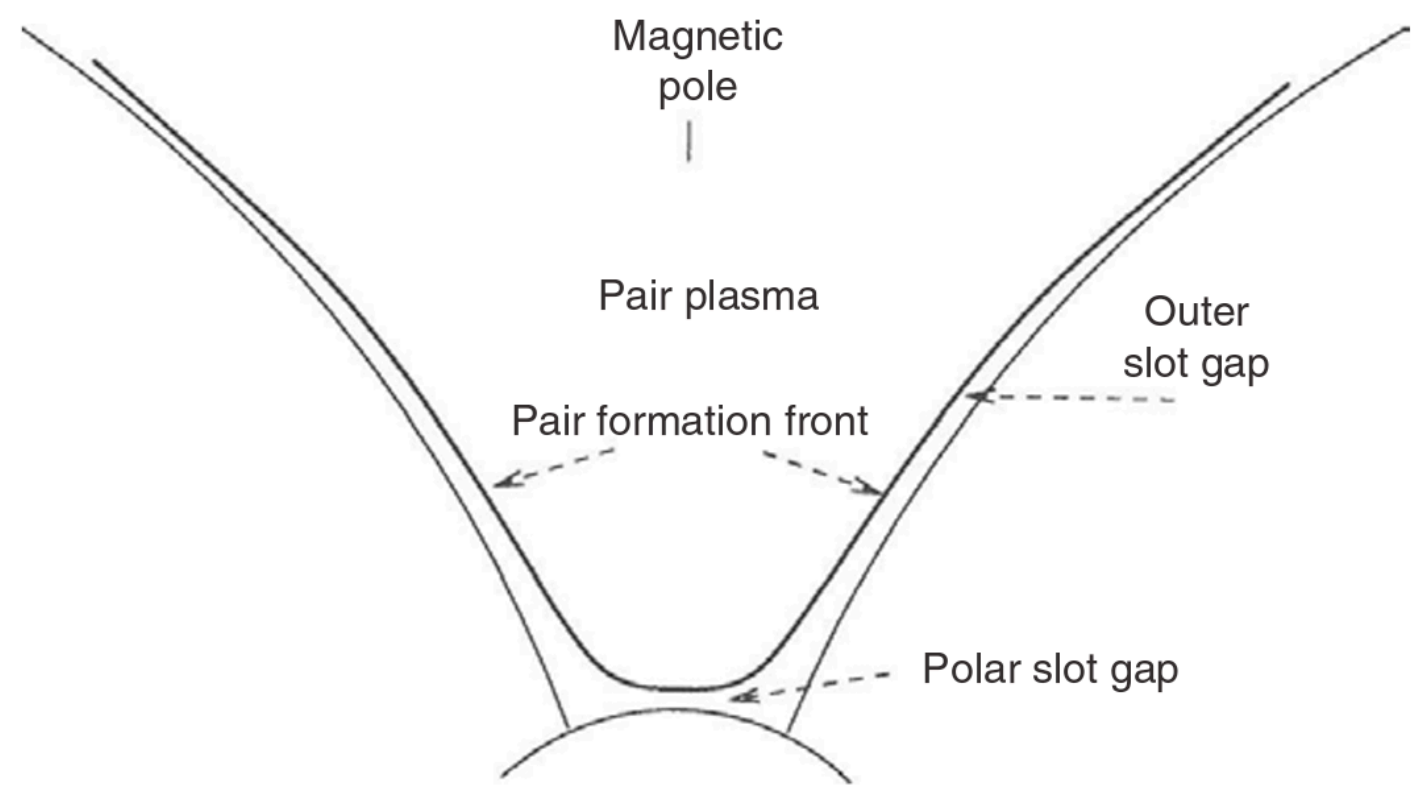

Figure 1.3: Location of the acceleration gaps responsible for most of the pulsar's observed emission. The outermost curved lines show the extent of the open field line region, while the pair formation front is the region inside of which pair formation cancels the charge depletion which causes the acceleration of charged particles in the gap, forming a force free region containing a pair plasma. (Image courtesy of Lyne \& Graham-Smith, 2012)

These gaps are called the "outer slot gap" and the "polar slot gap" (or simply the "outer gap" and "polar gap"), are shown in Figure 1.3, and are thought to be the seat of much of a pulsar's electromagnetic emission. 


\subsubsection{Acceleration Gaps}

\subsubsection{Polar Gap}

As the charged particles are flowing out from the surface of the neutron star into the magnetosphere, there will be a region where the charge density of the magnetosphere falls below the density required to maintain a force-free condition close to the surface of the neutron star and bounded by the last open magnetic field lines that defines the open field line regions. This region is known as the polar gap, and it is especially important in part because it is located in the area of the magnetosphere where the magnetic field strength is the highest.

In addition to one of the strongest magnetic fields we know of, the particles emanating from the neutron star also are accelerated by an enormous electric field. An expression for this electric field at the surface of the neutron star (i.e. at $r=R$ is shown in Equation 1.14, however these charges are not only subject to this electric field at the surface of the stellar surface, but rather along the entirety of their trajectory into the force-free region of the magnetosphere. As a function of the height above the polar cap, $z$, the strength of this electric field is

$$
E_{\|}(z)=\frac{2 \Omega B_{S}\left(h_{g a p}-z\right)}{c}
$$

where $h_{\text {gap }}$ is the height of the polar gap.

We can find the potential $V$ due to this electric field, and determine the total potential drop across the gap to be 


$$
\Delta V_{\text {gap }}=\frac{\Omega B_{S} h_{\text {gap }}^{2}}{c} .
$$

Particles traveling through this electric field easily gain relativistic energies, as for typical pulsar parameters $\left(\Omega=2 \pi / P=2 \pi \mathrm{rad} / \mathrm{s}, B_{S}=10^{12} \mathrm{G}\right.$, and $h_{\text {gap }}=10^{3}$ $\mathrm{cm})$, the total energy gained, $e \Delta V \sim 10^{11} \mathrm{eV}$. This corresponds to a Lorentz factor of

$$
\begin{gathered}
E_{\text {particle }}=e \Delta V \\
\gamma_{\text {max }} m_{e} c^{2}=\frac{e \Omega B_{S} h_{\text {gap }}^{2}}{c}
\end{gathered}
$$

so

$$
\gamma_{\max }=\frac{e \Omega B_{S} h_{g a p}^{2}}{m_{e} c^{3}} .
$$

Again inputting typical pulsar parameters means that a charged particle crossing the whole of the polar gap will reach a speed of $\gamma_{\max } \sim 10^{7} !^{3}$

The magnetic field lines along which these particles travel will have some curvature described by a radius of curvature $R_{c}$, and will therefore emit curvature radiation. The frequency of this radiation will be

$$
E_{C R}=\hbar \omega=\frac{3 \gamma^{3} \hbar c}{2 R_{c}}
$$

If the energy of this photon exceeds the rest mass energy of an electron-positron pair $\left(E_{e p}=2 m_{e} c \approx 1 \mathrm{MeV}\right)$, it can produce such a pair. For a typical curvature

\footnotetext{
${ }^{3}$ This corresponds to a speed of $0.999999999999995 c$ !
} 
radius $R_{c}=10^{6} \mathrm{~cm}$, photons produced by the charged particles originating from the neutron star have energies of $E_{C R} \approx 800 \mathrm{MeV}$, thus $E_{C R} \gg E_{e p}$, and therefore electron-positron pairs are produced.

These pairs, called "secondary particles" (as opposed to "primary particles", which are the charged particles originating from the neutron star itself) are produced in the polar gap and are therefore accelerated by the same electric field as the primary particles. Thus, these particles can emit their own curvature radiation, which can in turn create further electron-positron pairs. This process is called a "pair cascade", and serves to significantly increase the particle density in the open field line region of the magnetosphere.

These secondary particles do not traverse the entire polar gap, and are therefore not accelerated to energies comparable to the primary particles, but will rather reach more modest speeds of $\gamma \approx 800$. This is intriguing, as (given a curvature radius of $R_{c}=10^{9} \mathrm{~cm}$ ) charged particles traveling at these speeds will emit curvature radiation with a frequency of $\sim 1 \mathrm{GHz}$, and is therefore a source of radio emission.

This, however, cannot entirely explain the radio emission of pulsars, as the high brightness temperatures seen in pulsars is inconsistent with the inefficiency of single-particle curvature radiation (Ruderman \& Sutherland (1975) estimated the fraction of the total energy radiated by a particle as it travels along a curved path to be $\left.\sim 10^{-13}\right)$. These particles must therefore emit coherently, though a physical model to explain how these particles gain coherence does not yet exist. Thus, a complete explanation of pulsar radio emission remains, for the time being, out of reach. 


\subsubsection{Outer Gap}

Away from the magnetic pole, the strength of the electric field that is responsible both for extracting primary particles from the neutron star surface and for accelerating those particles to high velocities is greatly reduced (see Equation 1.14), meaning primary particles will be able to travel farther into the magnetosphere before they are able to emit photons with energies high enough to undergo pair production. As a result, when sufficiently high energy photons are emitted from primary particles traveling along open magnetic field lines away from the pole, they will then be emitted in a region of the magnetosphere in which the magnetic field strength is also dramatically smaller than that which would be found in the polar gap (as both the angle with respect to the magnetic pole and height above the neutron star surface will both be non-negligible in this region). Since the pair production mean free path of an energetic photon depends strongly on the strength of the magnetic field in the region through which it propagates, the curvature radiation produced from primary particles in the outer edges of the open field line regions is much more likely to be able to leave the magnetosphere and therefore be detected by observers. Indeed, this phenomenology is reflected in multi-frequency analyses of pulsars, where pulsar $\gamma$-ray profiles are typically much broader than their radio counterparts, consistent with an emission region located farther away from the magnetic axis than the radio emission region.

Still, curvature radiation will be emitted across the outer gap, including in the region close to the neutron star surface. There is therefore a higher probability 
that pair production will occur in this region. Because this region is farther from the magnetic axis, however, the curvature radiation photons (which are originally emitted tangent to the magnetic field lines) can obtain non-negligible pitch angles with respect to the magnetic field lines. Therefore, when pairs are produced from this emission, they spiral around their local magnetic field lines, producing softer high-energy emission.

Observations of pulsars spanning a wide range of energies show evidence for the existence of multiple high-energy emission mechanisms. For example, observations of the Crab pulsar from keV to GeV energy ranges (Figure 1.4) show a clear distinction between hard and soft high-energy emission, consistent with the two-phenomena model outlined above.

Despite the simplicity of this model of the high-energy emission in pulsars, it explains the observed emission surprisingly well in that not only does it explain the wider pulsar profile seen at high energies, but also curvature radiation is bright enough on its own to explain the brightness of the emission we see at high energies, meaning we do not need to find some amplification mechanism, as we do for the radio emission.

\subsection{Pulsar Types}

Perhaps the most essential property of pulsars is the stability of their rotation. This stability allows us to predict when we will detect the next pulse from a pulsar and to model any discrepancy between our prediction and the reality of when we 


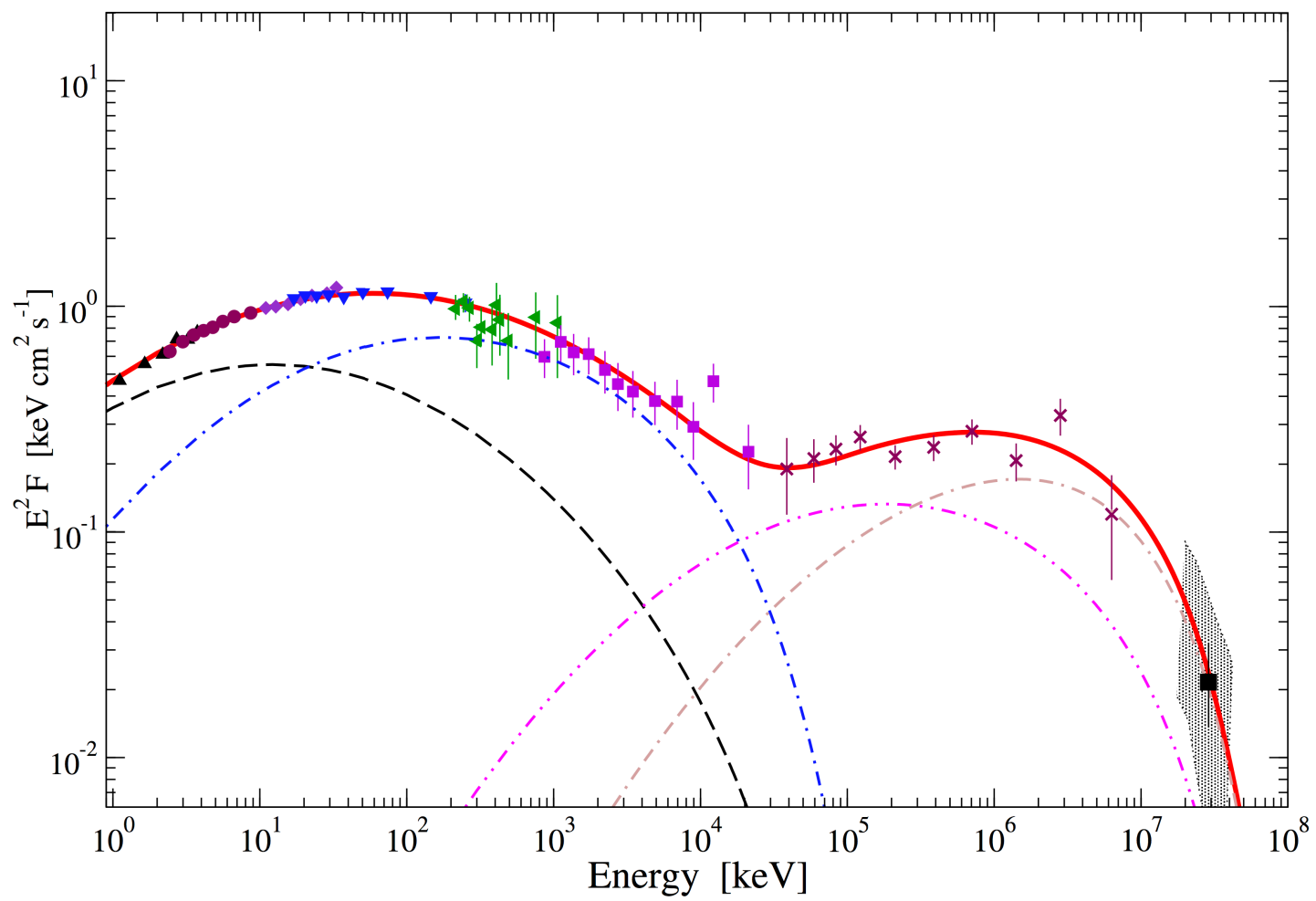

Figure 1.4: High-energy emission from the Crab pulsar. A clear break in the emission at energies of $\sim 2 \times 10^{4} \mathrm{keV}$ motivates a two-component model of the high-energy emission. Figure from Kuiper et al. (2003) 
observe the pulses. In practice, a myriad of effects can change the time of arrival of a pulse. Consider a pulsar in a binary system, for example. The Doppler effect will cause the observed pulse period to shift, depending on where the pulsar is in its orbit at the time of observation.

What may seem like an annoyance is in fact an enormous advantage, as modeling these discrepancies provides insight into the effects causing them. Keeping with the previous example, a simpler modeling of the orbit provides constraints on the binary system's orbital period, eccentricity, projected semi-major axis (which are products of the orbital radius and inclination angle), minimum companion mass, and epoch of periastron. This is but one of many examples of the power of the process called "pulsar timing".

As rich a subject as pulsar timing is, we can gain a considerable amount of understanding from a simple consideration of the most basic parameters to come from pulsar timing: the spin period and period derivative. The fact that characteristic values for fundamental pulsar parameters can be calculated from only the pulsar's spin period and spin period derivative (see Section 1.2, especially Equations 1.4, 1.9, and 1.11) is evidence of these parameters' importance, yet the efficacy of the $P-\dot{P}$ diagram (Figure 1.5) in providing a snapshot of the pulsar population is nonetheless remarkable, and the dependence of these fundamental characteristic values on $P$ and $\dot{P}$ allows us to view at a glance how the pulsar population is distributed with respect to them. Further, since one of these parameters is the characteristic age $\tau_{c}$ (see Equation 1.9) of a pulsar, we can compare old pulsars to young pulsars, giving us insight into how pulsars evolve with time. 


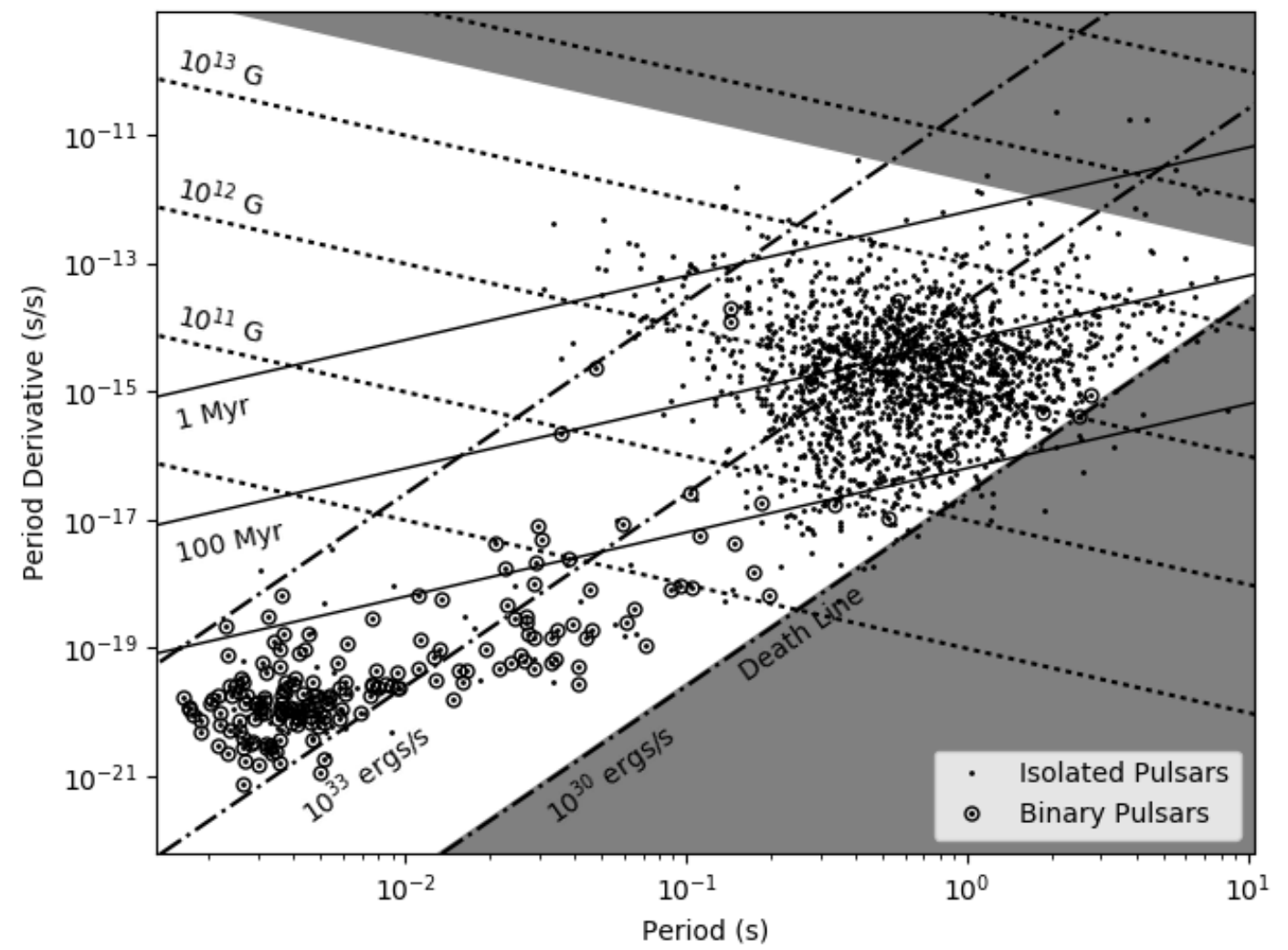

Figure 1.5: Spin period $P$ vs period derivative $\dot{P}$ for the current pulsar population. Lines of constant spindown luminosity, characteristic age, and magnetic field strength are plotted using Equations 1.4, 1.9, and 1.11 (respectively) and assuming typical pulsar parameters. Also plotted is the theoretical "death line", given by Equation 1.23. Note the clear distinction between pulsars with longer spin periods and higher period derivatives (canonical pulsars) and those with shorter spin periods and lower period derivatives (millisecond pulsars). 
Indeed, we see that the youngest pulsars (with $\tau_{c} \sim 10^{4} \mathrm{y}$ ), many of which are associated with supernova remnants, are born with relatively short spin periods $(P \lesssim 0.1 \mathrm{~s})$, high period derivatives $\left(\dot{P} \gtrsim 10^{-13}\right)$, and correspondingly high spin down luminosities $\left(\dot{E} \gtrsim 10^{36} \mathrm{ergs} / \mathrm{s}\right)$. As these pulsars continue to emit electromagnetic radiation via magnetic dipole radiation (see Equation 1.1), their spin periods increase, their spin period derivatives decrease, and they travel along lines of constant magnetic field strength and increasing $\tau_{c}$.

Pulsars continue this evolution until they reach a point whereby radio emission is no longer possible. This point is not well understood, but it is thought that this happens when the mean free path of a curvature radiation photon in a magnetic field exceeds some critical value, reducing the efficiency of the pair cascade process described in Section 1.2.2.1 and making radio emission effectively impossible. A comprehensive model describing this process is elusive, as an accurate model would be dependent on parameters that are extremely difficult to probe (such as the magnetic field structure near the surface of the pulsar in the polar gap, where higher-order moments of the magnetic field are thought to play a more significant role, see Chen \& Ruderman, 1993; Ruderman \& Sutherland, 1975). Still, a reasonable estimate of this death line is

$$
\dot{P} \geq 2.9 \times 10^{13} P
$$

(see Bhattacharya et al., 1992). The existence of pulsars across this "death line" highlights the fact that this line is an approximation, and that a complete theory of 
the pulsar death line is yet to be formulated.

Another way in which the efficiency of pair production can be greatly reduced (therefore effectively quenching a pulsar's radio emission) is if the magnetic fields through which the curvature radiation photons are traveling are too strong. In this regime, the photons can undergo magnetic photon splitting, whereby the photon splits into two lower-energy photons. In strong enough magnetic fields, this process can be extremely effective at keeping photons below the energy threshold required for pair creation. The magnetic field strength at which this process is believed to dominate is (Baring \& Harding, 1997; Baring \& Harding, 1998):

$$
B_{p s}=4.4 \times 10^{13} \mathrm{G} \text {. }
$$

Taking a more static view of the $P-\dot{P}$ diagram, we see that pulsars generally fall into one of two "clumps" on the $P-\dot{P}$ diagram: those with spin periods $P \sim 1 \mathrm{~s}$ and spin period derivatives $\dot{P} \sim 10^{-15}$ (called "normal" or "canonical" pulsars), and those with spin periods $P<0.03$ s and spin period derivatives $\dot{P} \sim 10^{-20}$ (called "millisecond" pulsars). We now turn our attention to these groups.

\subsubsection{Canonical Pulsars}

Canonical pulsars make up the bulk of the pulsar population. As of this writing, $\sim 86 \%(2261 \text { out of } 2613)^{4}$ of the pulsars currently discovered have spin periods $P>0.03$ s. Of these, 47 (just $2 \%$ ) are in binary systems, and 59 are

\footnotetext{
${ }^{4}$ Numbers from the ATNF pulsar database. See http://www . atnf .csiro.au/research/pulsar/psrcat/
} 
associated with supernova remnants, with these associations being biased towards young (i.e., relatively low $P$, high $\dot{P}$, and therefore low $\tau_{c}$ ) pulsars, as the supernova remnant dissipates with time. Canonical pulsars have high $\left(\sim 10^{12} \mathrm{G}\right)$ magnetic field strengths, and are much more likely to be found near the Galactic plane.

Since the radio emission is thought to originate above the polar cap (see Section 1.2.2.1) and since size of the polar cap is determined by the light cylinder radius (see Equation 1.18), we expect canonical pulsars, with their longer spin periods, to have much smaller polar cap regions and correspondingly narrower pulse profiles. Indeed, measured pulse widths are seen to decrease with increasing spin periods Lyne \& Manchester (1988), with the average fraction of the pulse profile where the pulsar is "on" being $\delta \sim 0.06$ for canonical pulsars (Lorimer et al., 2006).

\subsubsection{Millisecond Pulsars}

The fast-spinning cousins to canonical pulsars are millisecond pulsars (MSPs), which make up the remaining 347 known pulsars. In addition to the small spin periods that give MSPs their namesake, MSPs also have very small spindown rates, and as a result, the characteristic ages for MSPs can approach a Hubble time. This suggests that not only are MSPs distinct from canonical pulsars in a more fundamental way than only having different physical properties than canonical pulsars (such as magnetic field strengths and space velocities). Rather, this suggests that MSPs differ in the process of their formation.

One clue as the the difference in the formation scenario of MSPs with respect 
to canonical pulsars is the high fraction of MSPs in binary systems. We saw in the previous section that $\sim 2 \%$ of canonical pulsars are in binary systems, whereas $\sim 65 \%$ of MSPs (225 of 347) are found in binary systems. This suggests that a binary companion may play an integral role in the formation of MSPs, and is the primary motivation of the "recycling" formation scenario of MSPs (Alpar et al., 1982).

This scenario starts with a stellar binary system where the more massive star evolves, goes supernova, and forms a canonical pulsar. Assuming the binary system survives the explosion, the pulsar and the companion star continue to evolve normally, with the pulsar losing energy due to magnetic dipole radiation, spinning down, and perhaps even crossing the death line. The companion star, meanwhile, continues to burn its stellar fuel until it evolves into a red giant, at which point its outer layers expand to fill (and eventually overfill) its Roche lobe.

The matter that expands outside of the companion's Roche lobe is then able to be accreted by the pulsar and begins to inspiral onto the pulsar's surface, where it transfers its angular momentum to the pulsar, decreasing the pulsar's spin period (i.e., "spinning up" the pulsar) and weakening the pulsar's magnetic field (Bisnovatyi-Kogan \& Komberg, 1974; Shibazaki et al., 1989). Since this process can revive a canonical pulsar that has crossed the death line, it is referred to as recycling, and can last as long as the companion star remains a giant. For larger mass companions, this can be a relatively small amount of time, leaving an MSP with a (relatively) long spin period. In this instance, since the companion is a high mass star, the companion itself may undergo a supernova explosion, either disrupting the 
system (leading to long period, isolated MSPs), or transforming it into a double neutron star system.

For smaller mass companions, this recycling stage can be much more lengthy. In this circumstance, the pulsar has the opportunity to accrete more matter, spinning up to the smallest spin periods known $(P<1.4 \mathrm{~ms})$. The companion star, meanwhile continues to evolve normally, leaving behind a neutron star-white dwarf system.

\subsubsection{Black Widow and Redback Pulsars}

There remains a major unanswered question regarding the previous MSP formation scenario: how are isolated, fast-spinning MSPs formed? The very fact that they are MSPs indicates that they started out in binary systems, and the fact that they have been spun up to such extremely low spin periods indicates that their companion must be a long-lived (i.e. low-mass) star, which precludes a supernova disrupting the binary system. Barring a gravitational interaction with another ob-

ject, there is no other obvious mechanism to disrupt the binary, and yet we see a number of fast-spinning MSPs that do not have binary companions.

An intriguing possibility was realized with the discovery of PSR B1957+20

(Fruchter et al., 1988b), a fast-spinning $(P=1.6 \mathrm{~ms}) \mathrm{MSP}$ in a tight (projected semi-major axis of $\sim 0.09 \mathrm{lt} \mathrm{s}$ ), nearly circular (orbital eccentricity $<4 \times 10^{-5}$, (Arzoumanian et al., 1994)) orbit, with a very low mass $\left(M_{c} \sim 0.02 M_{\odot}\right)$ companion. This system showed radio eclipses when the pulsar was at superior conjunction (i.e., 
on the far side of the companion) that lasted $\sim 10 \%$ of the duration of the orbit. The length of this eclipse shows that it is not due to simple occultation by the companion star itself, and the fact that pulse delays are observed just before and after the eclipse implies that it is likely due to an abundance of ionized gas in the binary system.

Further observations of the companion star at optical wavelengths showed significant variation of the total intensity of the companion at the orbital period of the system (Fruchter et al., 1988a), that the side of the companion facing the pulsar was 2-3 times hotter than the side facing away from the companion, and that the companion was nearly filling its Roche lobe (Reynolds et al., 2007).

The obvious next step was X-ray observations. Pulsars are X-ray sources if for no other reason than they are hot enough to emit significant blackbody emission in the X-rays. Yet their X-ray emission can be much more rich: heated polar caps can cause pulsed thermal emission, and synchrotron emission from the outer gap can cause pulsed magnetospheric emission (see Section 1.2.2.2). X-ray observations of PSR B1957+20 (Stappers et al., 2003), however, found that its X-ray spectrum included a hard, non-thermal component that was modulated at the orbital period of the system. This modulation included an X-ray eclipse occurring when the pulsar was at superior conjunction (therefore coinciding with the radio eclipse).

The totality of these characteristics suggests that the companion star is being shocked and heated by the pulsar. Since the companion star is nearly filling its Roche lobe, the material interacting with the pulsar wind (i.e. the outer layers of the companion) are relatively weakly gravitationally bound to the companion, 
making it possible for the pulsar's energetic wind to ablate the companion, leaving a highly recycled, isolated MSP. For this reason, they are called "black widow" pulsars, after the spiders that cannibalize their mates.

Since the discovery of PSR B1957+20, many more systems with similar characteristics (highly recycled MSPs in tight, circular orbits around very low mass companions) have been discovered. These systems tend to possess similar orbital characteristics to the original black widow pulsar, and have therefore proven to be an important probe into MSP evolution, although it is still unclear whether the mass loss rate from these systems is sufficient for the companion stars to be completely ablated in a reasonable amount of time.

Another important piece of the MSP evolutionary timeline comes in the form of systems that are similar to, yet distinct from their black widow cousins. These systems are named "redbacks", after the Australian cousin to the black widow spider, and like black widows, these systems exhibit radio eclipses and are found in tight binary systems. Unlike black widows, however, their companions are considerably more massive $\left(M_{c}=0.2-0.4 M_{\odot}\right)$, and tend to be non-degenerate (Roberts et al., 2014). As with black widow companions, redback companions also tend to be nearly Roche lobe filling, although the fact that redback companions are larger means that they have a larger cross-section with which to be shocked by the pulsar's wind, meaning the shock luminosities of redback systems tend to be higher than those seen in black widow systems.

These systems, therefore, are thought to represent MSPs in the stages immediately following their accretion, before the pulsar has had the opportunity to 
ablate the companion to any considerable degree. This idea is not without support. For example, PSR J1740-5340 is in an orbit around a nearly Roche lobe filling $M_{c}=0.22-0.32 M_{\odot}$ companion (Sabbi et al., 2003) showing radio eclipses during around $40 \%$ of the orbit (D'Amico et al., 2001) and a reduction of X-ray flux near superior conjunction (Grindlay et al., 2002), making it a typical redback system. Further optical analysis (Mucciarelli et al., 2013), however, showed that the companion contained levels of carbon and nitrogen that are incompatible with the local main sequence population, and therefore is likely an evolved, more massive main sequence star whose outer layers have been peeled by accretion, leaving exposed the inner, more metal-rich layers of the star.

Another interesting system is the system first discovered as FIRST J102347.6+003841. This system showed variability in the intensity of its optical emission coupled with Hydrogen and Helium emission lines, causing it to first be classified as a cataclysmic variable (Bond et al., 2002). Subsequent observations, however, showed that these emission lines disappeared completely, and the remaining emission was more characteristic of a G-type star (Thorstensen \& Armstrong, 2005). Further analysis revealed that this system was a $\sim 4.8$ hour binary system, and radial velocity measurements implied that the more massive star was a neutron star orbiting a companion with $M_{c} \sim 0.2 M_{\odot}$. All of this pointed to FIRST J102347.6+003841 being a low-mass X-ray binary, that is, a neutron star in the process of accreting matter from its companion. This scenario was confirmed when Archibald et al. (2009) detected pulsed radio emission with a period of $P \sim 1.69 \mathrm{~ms}$. This emission showed frequency-dependent orbital eclipses and X-ray studies of the 
system (Archibald et al., 2010) showed orbitally modulated X-ray emission with a hard, non-thermal component. Evidently, PSR J1023+0038 was the first pulsar ever to be detected transitioning from an accretion stage to a redback stage, giving credence to the idea that redbacks represent MSPs in the stages immediately following their accretion.

\subsection{Thesis Outline}

Pulsar polarization and the precise calibration thereof is an important component in many facets of radio pulsar astronomy. A pulsar's polarimetric characteristics can tell us about the structure of its magnetic field and the location of the emission regions within the pulsar magnetosphere. In some cases, it is also possible to determine the spin geometry of the pulsar using its polarimetric characteristics. The polarimetric calibration of radio telescopes, then, is incredibly important, not only to facilitate the aforementioned analyses, but also because the lack of precise polarimetric calibration can artificially change the shape of the pulse profiles, the accurate determination of which lies at the heart of pulsar timing.

In light of its importance to pulsar astronomy, I present an overview of the basics of radio polarimetry in Chapter 2. I start with a toy receiver, describe how it would detect different orientations of incoming light, and use those examples to describe the Stokes Parameters. I then introduce the Poincaré sphere, and describe its utility as a visualization tool for radio polarimetry. Next, I describe in detail the Mueller Matrix, discussing the possible origins and effects of its components. This 
leads to a discussion of the different methods of polarimetric calibration, including the Ideal Feed Assumption and the determination of the full Mueller Matrix. Lastly, I describe some of the uses for radio polarimetry, namely, the Rotating Vector Model and Faraday Rotation.

Chapter 3 uses this background in radio polarimetry to describe the polarimetric dataset of the North American Nanohertz Observatory for Gravitations waves (NANOGrav) as taken with the Arecibo Observatory. The product of this work is the most sensitive polarimetric dataset to date. The sensitivity of the dataset allows for the first detection of "microcomponents", or pulse profile components that have intensities hundreds of times lower than the main pulse peak. I also determine Faraday rotation measures and use them to describe the magnetic field of the Galaxy. I also describe the implementation of a new scheme to calibrate the data. This scheme allows the polarimetric response of Arecibo's receivers to be characterized using short pulsar observations, which makes it possible to characterize the receiver on an epoch-by-epoch basis. I then analyze these polarimetric responses and find evidence that the receiver responses are changing with time.

Next, I move on to analyses of spider pulsars, which are recently recycled pulsars believed to be ablating their companions. These systems are thought to be the evolutionary link between canonical pulsars and isolated millisecond pulsars. The ablation process is likely to be caused by the pulsar wind interacting with the companion star, an interaction which is expected to generate X-ray emission which is distinct from the pulsar's blackbody emission. Characterizing this emission is essential to understanding the pulsar-companion star interaction, and thereby the 
process through which MSPs become isolated.

The first of these analyses are presented in Chapter 4, where I describe the first X-ray observations of five spider pulsars with the Chandra X-Ray Observatory. I analyze spectral data from these observations to search for a non-thermal component to the X-ray spectra, and present the X-ray emission versus orbital phase for these sources to search for orbital modulation characteristic of an intrabinary shock.

Chapter 5 discusses the X-Ray observations of three spider pulsars, this time taken with XMM-Newton. Two of these sources are new, while the other was the most promising candidate for intrabinary shock emission from the work in Chapter 4. I present spectral fits for these sources, including fits using different models of the neutron star blackbody emission. I also analyzed the X-ray emission versus orbital phase for these sources, and for one source am able to fit Gaussian components to the X-ray emission. 


\section{Chapter 2}

\section{Pulsar Polarimetry}

The progress that has been made thus far, and progress to be made in the future, towards understanding the fundamental nature of pulsars will certainly be contingent upon the ability to observe them with a high degree of precision. Such precision makes polarimetric calibration necessary. We therefore turn our attention to calibration, and start the discussion with an overview of the basics of radio polarimetry.

\subsection{Radio Polarimetry}

\subsubsection{Toy Receiver and Stokes Parameters}

Let us assume that we would like to observe some radio source with unknown polarization properties. A simple line feed would allow us to sample the electric field emitted from the source, since an electric field incident on such a feed would induce a current (and therefore a voltage) in the feed. The problem arises, though, if we imagine the light emitted from our source to be completely polarized and perpendicular to our line feed. Since our simple detector will only be sensitive to the component of the electric field that is aligned with the feed, we would not be able to detect light from this source at all. The simplest solution to this problem is to include another line feed that is orthogonal to the first (see Figure 2.1). Such 
a setup would allow us to completely sample the incoming electric field, since any component of the polarized light that is perpendicular to (and therefore undetectable to) one of the feeds would be aligned with (and therefore completely detected by) the other. Further, since the incoming electric field (and consequently the measured voltages) is complex, we also retain the phase information of the incoming light.

This setup is sensitive to all incoming light. Unsurprisingly, then, modern radio telescope receivers contain two orthogonal arms that sample the electric field in orthogonal directions (see Carl Heiles' description (Heiles, 2002) for more detail). These are able to detect all of the light at frequencies which they are sensitive to, and from the electric fields measured by the telescope, we can determine the polarization properties of that light. These properties are described by the four Stokes Parameters, which we can use the toy receiver to define.

If we define the two orthogonal directions over which the telescope samples the electric field as $X$ and $Y$, and the time-averaged field being detected in those directions as $E_{X}$ and $E_{Y}$ with their complex conjugates denoted as $E_{X}^{*}$ and $E_{Y}^{*}$ respectively, the Stokes Parameters are:

$$
\begin{gathered}
I=E_{X} E_{X}^{*}+E_{Y} E_{Y}^{*} \\
Q=E_{X} E_{X}^{*}-E_{Y} E_{Y}^{*} \\
U=E_{X} E_{Y}^{*}+E_{X}^{*} E_{Y} \\
i V=E_{X} E_{Y}^{*}-E_{X}^{*} E_{Y} .
\end{gathered}
$$

In principle, these parameters completely describe the incoming light. Completely polarized, completely unpolarized, or somewhere in between, it accounts for 


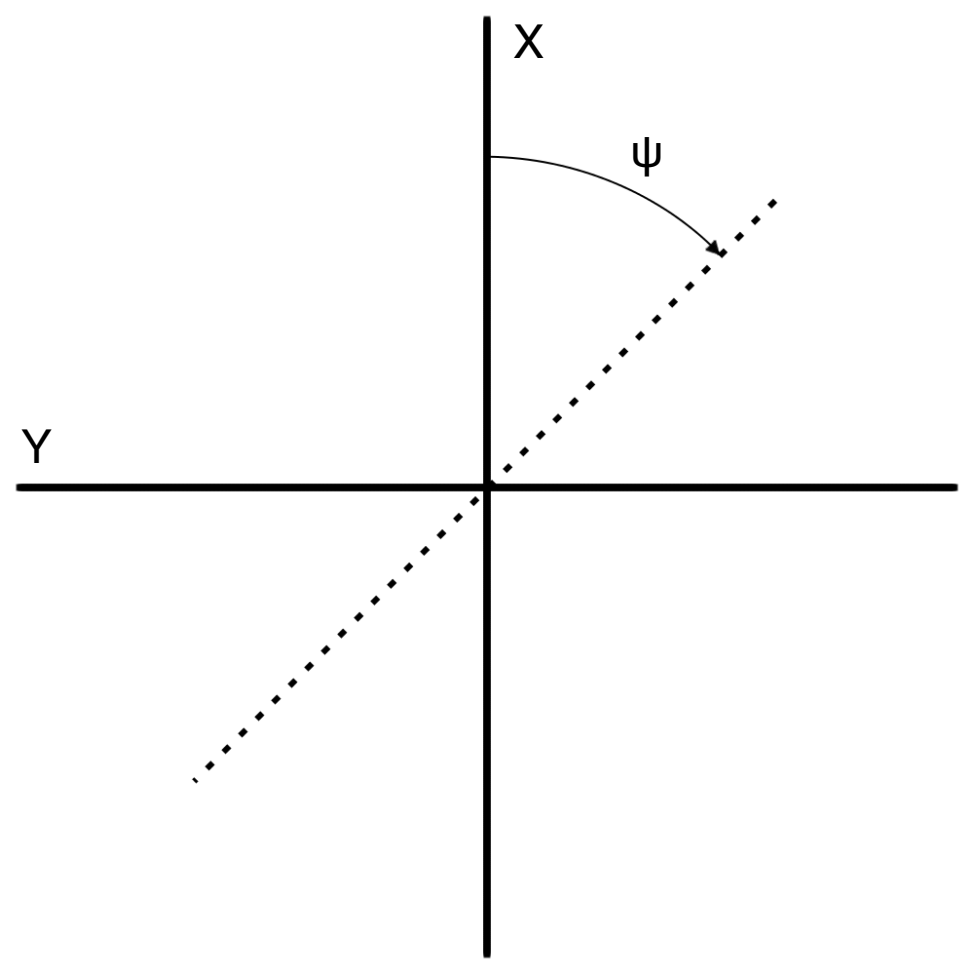

Figure 2.1: Toy radio receiver. As described in Section 2.1.1, this receiver, which has two orthogonal feeds $(X$ and $Y$ ), is sensitive to all incoming light, regardless of the angle of polarization, $\Psi$. 
all incoming light as well as characteristics such as the direction of linear and circular polarization.

Though mathematically convenient, the Stokes Parameters may seem overly cumbersome in that they seem to transform something that is relatively easy to visualize (an oscillating electric field traveling through space) into something that is nearly impossible to visualize (sums and differences of complex auto- and crosscorrelations). Indeed, the considerable advantages of the Stokes Parameters (their completeness, the ability to build receiver components that automatically generate them, their use in polarimetric calibration) do come at the cost of familiarity.

Still, the advantages are considerable, so it is worth taking the time to try to gain some familiarity with the Stokes Parameters. In that spirit, let us consider some examples:

First, recall our toy receiver: two orthogonal line feeds, one oriented in the $X$-direction, and one in the $Y$-direction. Next, consider how our receiver would behave if an monochromatic radio wave were incident on the receiver such that the electric field was perfectly aligned with our $X$ receiver arm: we would detect the electric field in the $X$ feed (i.e. $E_{X}=E$ ) and would detect nothing in the $Y$ feed (i.e. $E_{Y}=0$ ). From Equation 2.1, it is easy to see that in this example, $U=V=0$, while $I=Q=2 E_{X}^{2}=|E|^{2}$. Note that if we had decided that the incident electric field was aligned with the $Y$ feed rather than the $X$ feed, we would still see that $U=V=0$, but now $I=Q=-2 E_{Y}^{2}=-|E|^{2}$. This seems to imply a general rule: Stokes Q describes the light polarized in a direction that is aligned with the receiver feeds, and the sign of Stokes Q describes which feed the polarized light is aligned 
with.

It is important not to get carried away, however. It is tempting to then think of Stokes Q as describing the linearly polarized light incident on the receiver, when this is not the case, as we see from our next example.

Consider how our receiver would behave if an monochromatic radio wave were incident on the receiver such that the electric field was perfectly misaligned with both the $X$ feed and the $Y$ feed (that is, the electric field is oriented at a $45^{\circ}$ angle with respect to both feeds). In this example, we would detect the electric field equally in both feeds. Therefore, $E_{Y}=E_{X}=|E| / \sqrt{2}$. Since $E_{Y}=E_{X}$, we see from Eq 2.1 that $Q=0$. By the same equation, though, we see that (since $E_{Y}$ and $E_{X}$ are in phase for linearly polarized light), $U=2 E_{X} E_{Y}=|E|^{2}$. Note that this is the same as Stokes Q in our first example, as the two examples are equivalent up to a rotation of the receiver.

It is then apparent that total linear polarization is neither Stokes Q nor Stokes $\mathrm{U}$, but rather the sum of the two. That is, the total linear polarization

$$
L=\sqrt{Q^{2}+U^{2}}
$$

Further, we are usually interested in the direction of the linear polarization. Since the magnitude of Stokes Q and U changed in our examples as the orientation of the incoming light changed with respect to the receiver feeds, we expect the orientation of the incoming light to be dependent on Stokes Q and U, and indeed, 


$$
\Psi=0.5 \tan ^{-1} \frac{U}{Q}
$$

where $\Psi$ is the position angle (PA) of the linearly polarized emission, which describes the orientation of the linear polarization. Here, the PA is actually the angle with respect to some nominal position, conventionally with respect to celestial north.

Finally, let us consider an electric field whose $X$ component lags its $Y$ component by $90^{\circ}$ in phase. In this instance, $E_{X}=-E_{X}^{*}=|E| / \sqrt{2}$, while $E_{Y}=E_{Y}^{*}=$ $|E| / \sqrt{2}$. Again, using Eq 2.1, we see that $Q=U=0$, whereas $I=V=2 E_{Y}^{2}=|E|^{2}$. Note that we could have stipulated that the $\hat{y}$ component lags its $\hat{x}$, and if this were the case, we would have $E_{X}=E_{X}^{*}=|E| / \sqrt{2}$, while $E_{Y}=-E_{Y}^{*}=|E| / \sqrt{2}$. We would then again find that $Q=U=0$, whereas now $I=-V=2 E_{Y}^{2}=|E|^{2}$. Clearly then, the magnitude of Stokes V describes the magnitude of the circular polarization, while its sign describes the handedness of the circular polarization ${ }^{1}$.

Having described both the linear and circular polarizations in terms of the Stokes Parameters, we can then describe the total polarization

$$
P=\sqrt{L^{2}+V^{2}}=\sqrt{Q^{2}+U^{2}+V^{2}},
$$

and the degree of polarization

$$
\frac{P}{I}=\frac{\sqrt{Q^{2}+U^{2}+V^{2}}}{I}
$$

\footnotetext{
${ }^{1}$ We are intentionally ambiguous here regarding what one calls "right-handed" and what one calls "left-handed" of the circular polarization since these labels do not have any bearing on the fundamental understanding of the Stokes Parameters. For a description of the convention used in the analyses presented in this work, see Chapter 3.
} 
A useful way of representing the polarization state is by way of a column vector. That is,

$$
S=\left[\begin{array}{l}
I \\
Q \\
U \\
V
\end{array}\right] .
$$

Normalizing this vector by the total intensity I would allow us to view the fractional polarization $\mathrm{P}$ as a vector in 3-dimensional space. Then the polarized emission from a source can be described as a point on the so-called Poincaré sphere.

The Poincaré sphere is a useful visualization tool for a few reasons: firstly, it can make the sometimes challenging problem of visualizing the polarization state of light resulting from the superposition of two polarized waves more readily graspable. This situation may arise when observing sources of non-negligible depth whose polarization properties change with respect to the line of sight, or when dealing with effects like Faraday depolarization (see Section 2.3). For examples like these, one can find the polarization state of the composite light by simply summing the polarization vectors of the components being superimposed. Note that this is not fundamentally different from simply summing the Stokes vectors of the superimposed light, however it provides a more natural framework for understanding the results.

Another reason that the Poincaré sphere is a useful visualization tool comes from the realm of polarimetric calibration. There are a number of ways to character- 


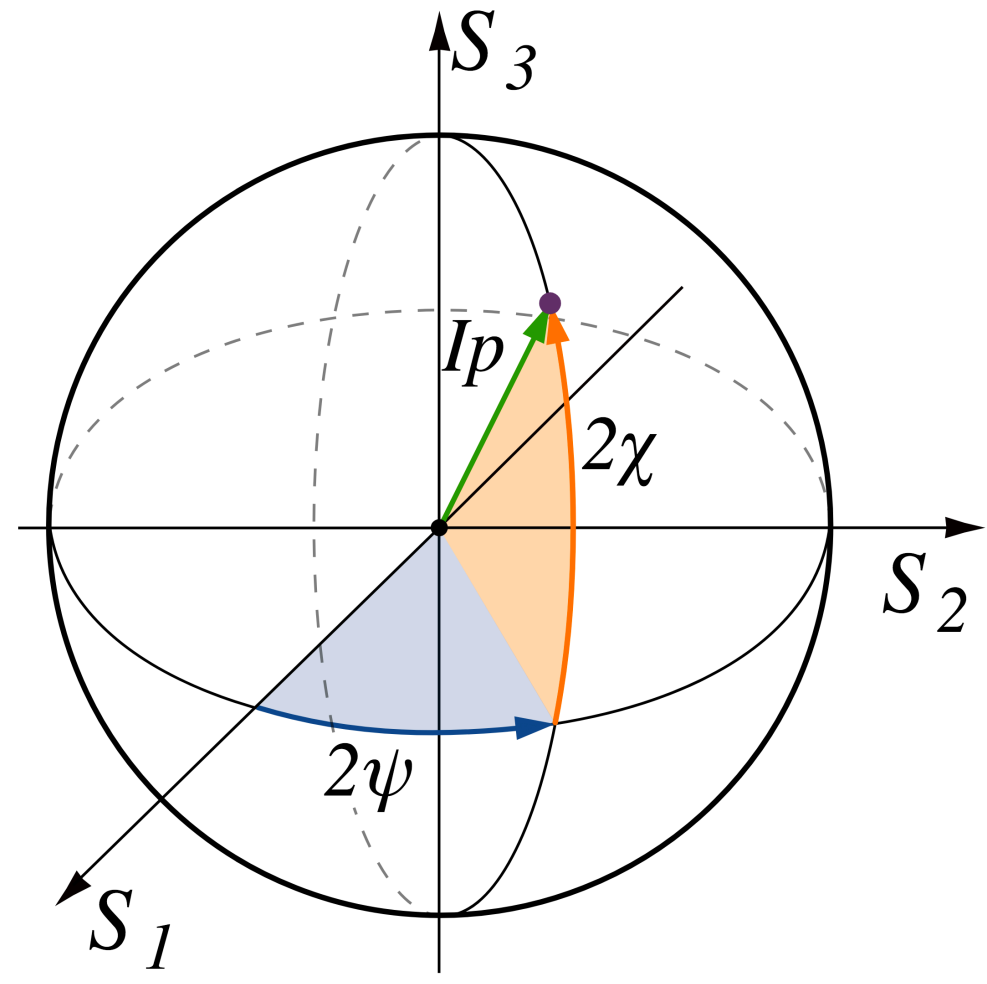

Figure 2.2: The Poincaré sphere: a visualization of the polarized light from a source. Three stokes parameters, $Q, U$, and $V$ (labeled here as $S_{1}, S_{2}$, and $S_{3}$, respectively, are plotted on orthogonal axes. Also shown are the total polarized intensity (labeled here as $I_{p}$ ), polarized position angle $\Psi$ (see Equation 2.3), and polarization ellipticity $\chi$.

ize the ways that a telescope will change incoming radiation, but some of the most useful (both in terms of talking about polarimetric calibration itself and as it relates to other pulsar observation considerations) stem from talking about polarimetric calibration as it relates to the Poincaré sphere. Indeed, the effects of polarimetric miscalibration can be thought of as spurious boosts and rotations along and about the three axes of the Poincaré sphere.

To illustrate this, we discussed earlier how linearly polarized light that was aligned and misaligned would each be described by the Stokes parameters (the former with Stokes Q and the latter with Stokes U). We then mentioned that this 
example was performed in the guise of a fixed receiver viewing two differently oriented polarizations of light, however we could equivalently view this example as a receiver that was rotated with respect to some fixed source of polarized light. Evidently, if we were to make some error in calibration whereby the absolute angle of the receiver were mistakenly calculated, it would serve to turn inherent Stokes Q into Stokes U (and back to Stokes Q, if the miscalculation were large enough). This specific miscalibration could then be described as a rotation of the Poincaré polarization vector about the Stokes $\mathrm{V}$ axis.

Another example can be gleaned from considering the effects of uncorrected differential gain (whereby one of the receiver feeds has a larger gain with respect to the other). A receiver suffering this particular affliction will tend to add Stokes Q to whatever it detects. This translates to a boost of the Poincaré polarization vector along the Stokes Q axis.

Note an inherent difference in these types of miscalibration: one (the rotation about the Stokes $\mathrm{V}$ axis) does not change the length of the Poincaré polarization vector, whereas the other (the boost along the Stokes Q axis) does change the length of the Poincaré polarization vector. This is important, as radio pulsar astronomers may find themselves in a situation where they are only concerned with the total intensity (Stokes I) from their observations, yet would still like to quantify the effect of insufficient polarimetric calibration on those observations. These astronomers will then be most interested in spurious receiver effects that will tend to boost the polarization vector. 


\subsubsection{Mueller Matrices}

Although we have spent some time talking about the Poincaré sphere and the polarization vector that lies within it, the more crucial quantity is certainly the Stokes vector, since, again, it provides a complete characterization of the light seen by the telescope. We are, of course, unconcerned with the light seen by the telescope, and are only concerned with the light emitted by the source. This distinction may seem pedantic, but in reality, those two quantities may be drastically different. We therefore turn our attention to polarimetric calibration.

The mathematical framework of polarimetric calibration is simplified by the Stokes vector, as the effects the telescope visits upon the incoming light can be represented as a $4 \times 4$ matrix $M$ (called the Mueller Matrix) acting upon the intrinsic Stokes vector emitted by the source. That is,

$$
S_{\text {meas }}=M S_{\text {int }}
$$

Generally, the Mueller Matrix takes the form,

$$
M=\left[\begin{array}{cccc}
m_{I I} & m_{Q I} & m_{U I} & m_{V I} \\
m_{I Q} & m_{Q Q} & m_{U Q} & m_{V Q} \\
m_{I U} & m_{Q U} & m_{U U} & m_{V U} \\
m_{I V} & m_{Q V} & m_{U V} & m_{V V}
\end{array}\right],
$$

where each element $m_{j k}$ represents the coupling the of the $j^{\text {th }}$ Stokes parameter to the $k^{\text {th }}$ Stokes parameter. 
In order to gain a more intuitive understanding of the Mueller Matrix, and to explain the parameters that might be found in a typical parameterization of the Mueller Matrix, we now take some examples. It is important to say at the onset, though, that because we have been talking about light incident on a toy receiver, it is natural to continue with this model when talking about polarimetric calibration and in the process, we will refer to various physical imperfections to understand how they will affect the Mueller Matrix and thereby the detected light. We stress at this point, though, that the physical imperfections discussed here are sufficient, but not necessary, explanations of any terms present in the Mueller Matrix. In fact, we usually consider the telescope receiver to be largely a black box, eschewing any specific physical interpretation of the telescope's effect on incoming light.

With that in mind, let us call to mind an example we have already talked about: that of the receiver being rotated with respect to the incoming light. In the previous section, we saw that the result of this effect is to transform Stokes $Q$ to Stokes $U$ (and vice versa). We also saw that because of the way Stokes $Q$ and $U$ are defined, we need only to rotate the receiver by $45^{\circ}$ in order to completely convert incoming Stokes Q to Stokes U. It should therefore be unsurprising that the matrix that describes this is a rotation matrix:

$$
M_{F O}=\left[\begin{array}{cccc}
1 & 0 & 0 & 0 \\
0 & \cos (2 P A) & \sin (2 P A) & 0 \\
0 & -\sin (2 P A) & \cos (2 P A) & 0 \\
0 & 0 & 0 & 1
\end{array}\right] \text {, }
$$


and this this matrix, when applied to the Stokes vector, will indeed transform Stokes Q to Stokes U (and vice versa). Here, we use the subscript "FO" to emphasize that this matrix describes a "Feed Orientation"-like transformation of the Stokes vector. Further, since the angle through which the feed rotates with respect to the source is called the "parallactic angle", we denote this angle as "PA" in the Mueller Matrix. In the previous section, we mentioned that although such a rotation changes the Stokes vector, it does not change the total intensity detected by the telescope. What we discussed in the context of the Poincaré sphere we can now see in the context of matrices: $M_{F O}$ will have no effect on the total intensity (i.e., Stokes I) of the incident light.

The other example we discussed involved an effect called differential gain, which can describe a situation in which one receiver feed has a higher gain with respect to the other. Since Stokes Q is detected from the difference of the powers in the telescope feeds (see Equation 2.1), the existence of nonzero differential gain means the telescope will erroneously detect Stokes Q. This translates to:

$$
M_{D G}=\left[\begin{array}{cccc}
1 & \frac{\gamma}{2} & 0 & 0 \\
\frac{\gamma}{2} & 1 & 0 & 0 \\
0 & 0 & 1 & 0 \\
0 & 0 & 0 & 1
\end{array}\right] .
$$

Here, we have used the subscript $D G$ to denote the matrix corresponding to the differential gain. This clearly serves to couple Stokes I to Stokes Q, and, as discussed previously, serves to change the total intensity of the detected light and is therefore 
an important consideration even if the observer is not particularly concerned with the polarimetric properties of the source they are observing.

As the incoming signals travel through the amplifier chains typically present in telescope receivers, it is possible for the signal from one of the feeds to be delayed with respect to the other. Although it is possible to cause such a delay in a multitude of ways, the most basic example would be a difference in the physical path length through which the signals must travel ${ }^{2}$. Such an effect would cause intrinsic Stokes $\mathrm{U}$ (i.e., light detected by both feeds in phase with each other) to be detected as Stokes $\mathrm{V}$ (i.e., light detected by both feeds $90^{\circ}$ out of phase with each other). If the signal is delayed by a phase of $\Psi$, the corresponding matrix will look like:

$$
M_{D P}=\left[\begin{array}{cccc}
1 & 0 & 0 & 0 \\
0 & 1 & 0 & 0 \\
0 & 0 & \cos (\Psi) & -\sin (\Psi) \\
0 & 0 & \sin (\Psi) & \cos (\Psi)
\end{array}\right],
$$

where the subscript $D P$ is to signify that the matrix corresponds to the differential phase. This matrix is a simple rotation matrix that, as expected, rotates Stokes U into Stokes V (and vice versa).

Since Equations 2.10 and 2.11 both deal with the amplifier chain, they're typically written together as:

\footnotetext{
${ }^{2}$ Caused for example by using cables of different length. We mention this to stress the sensitivity of the receiver to various effects, and therefore the appeal of the "black box" view of the receiver.
} 


$$
M_{A}=\left[\begin{array}{cccc}
1 & \frac{\gamma}{2} & 0 & 0 \\
\frac{\gamma}{2} & 1 & 0 & 0 \\
0 & 0 & \cos (\Psi) & -\sin (\Psi) \\
0 & 0 & \sin (\Psi) & \cos (\Psi)
\end{array}\right] .
$$

So far, the effects we have been considering have been those in which the telescope feeds have been sampling the correct component of the incident electric field, however, components in the receiver changed the signal so that the correctly detected emission was either delayed or amplified by some factor. The last effect we discuss is called cross-coupling, and it describes the situation where one feed spuriously detects emission that should only be detected in the other.

To illustrate a circumstance that could lead to such a situation, consider again our toy receiver, with the incident light oriented such that the electric field aligns perfectly with one of the orthogonal feeds (the $X$ feed, for example). We know that with this setup, our toy receiver would detect only Stokes Q and will not detect any Stokes $\mathrm{U}$ because the electric field projected onto the $Y$ receiver is zero. Now imagine that the receiver is defective such that the feeds are not perfectly orthogonal, and therefore the $Y$ feed is at some nonzero angle to a line orthogonal to the $X$ feed. If this were the case, the component of the electric field projected on to the $Y$ receiver would no longer be zero. As a result, the receiver would still detect the Stokes Q, but would now also detect Stokes U.

An added layer of complexity is necessary to describe cross-coupling, because we have also been assuming that the toy receiver is flat in the direction of the electric 
field's propagation (the $Z$ direction). If this were not true, it would also introduce a delay to the spurious, coupled emission detected in the $Y$ receiver $^{3}$, and, whereas before the receiver would detect spurious Stokes U, the phase delay would make the receiver detect spurious Stokes V instead.

This means that in order to account for this cross-coupling, we need to know both the magnitude and phase at which the cross-coupled emission is detected. The matrix describing cross-coupling is:

$$
M_{C C}=\left[\begin{array}{cccc}
1 & 0 & A & B \\
0 & 1 & C & D \\
A & -C & 1 & 1 \\
B & -D & 0 & 0
\end{array}\right],
$$

where

$$
\begin{aligned}
& A=\epsilon_{1} \cos \left(\theta_{1}\right)+\epsilon_{2} \cos \left(\theta_{2}\right) \\
& B=\epsilon_{1} \sin \left(\theta_{1}\right)+\epsilon_{2} \sin \left(\theta_{2}\right) \\
& C=\epsilon_{1} \cos \left(\theta_{1}\right)-\epsilon_{2} \cos \left(\theta_{2}\right) \\
& D=\epsilon_{1} \sin \left(\theta_{1}\right)-\epsilon_{2} \sin \left(\theta_{2}\right)
\end{aligned}
$$

Now that we've described the ways in which the receiver can change the incoming light, we need to make sure we combine these effects in the right way. Since we're dealing with matrices and matrix multiplication is not commutative, we must be careful about the order in which we combine the matrices defined above, and

\footnotetext{
${ }^{3}$ Note that this is different from the phase delay we described earlier where the correctly detected emission was delayed by some phase!
} 
the order in which the incoming light encounters these effects defines the order in which they are to be combined. First, the light encounters a rotated feed, then imperfections in the feed itself will cross-couple the emission, then this cross-coupled emission is sent through the amplifier chain. Therefore, we have:

For completeness, we show the full Mueller Matrix here:

$$
M=\left[\begin{array}{cccc}
1 & E H-J(A+E C) & J E+H(A+E C) & B+E D \\
E & H-J(A+E C) & J+H(A+E C) & D+E B \\
A F-G B & H(G D-F C)-J F & J(G D-F C)+H F & -G \\
A G+B F & -H(G C+F D)-J G & -J(G C+F D)+H G & F
\end{array}\right]
$$

where A, B, C, and D are as defined in equation 2.14 and

$$
\begin{gathered}
E=\frac{\gamma}{2} \\
F=\cos (\phi) \\
G=\sin (\phi) \\
H=\cos 2 P A \\
J=\sin 2 P A .
\end{gathered}
$$

It is easy to see why we chose to present the underlying components of the Mueller Matrix first!

Now that we are well-versed in the mathematics of polarimetric calibration, we are ready to discuss actual procedures used to calibrate data. 


\subsubsection{Ideal Feed Assumption}

For reasons that will become clear in the next section, the polarimetric calibration process can be greatly simplified by assuming our feed is perfect. Under this assumption, called the Ideal Feed Assumption (IFA), we can then ignore all the terms in equation 2.13. We note at this point that cross-coupling introduces spurious emission to the observation, so even if one is uninterested in the polarimetric properties of their source, the IFA should only be used if 1) the telescope has been fully calibrated before, and the cross-coupling of the feed has been found to be negligible and stable over time, or 2) the observer does not care about collecting precision data.

Calibrating under the IFA makes use of a signal injected into the receiver with a device called a noise diode. This ideally injects a pulsed square wave into both feeds equally and simultaneously. The output from such a signal should be only Stokes U, thus any detected Stokes Q or V can be used to determine the differential gain and phase of the amplifier chain. Also, as the noise diode ideally injects the signal at the same strength over the entire usable bandwidth of the receiver, the bandpass (i.e., total gain versus observing frequency) can be determined. Once these parameters have been determined, they can be used to calibrate a subsequent observation.

Inspecting the raw noise diode observation shown in Figure 2.3, we are able to see what the effects described above look like in real data. Bearing in mind that the injected signal is pure Stokes U, we see that we detect Stokes Q across the 

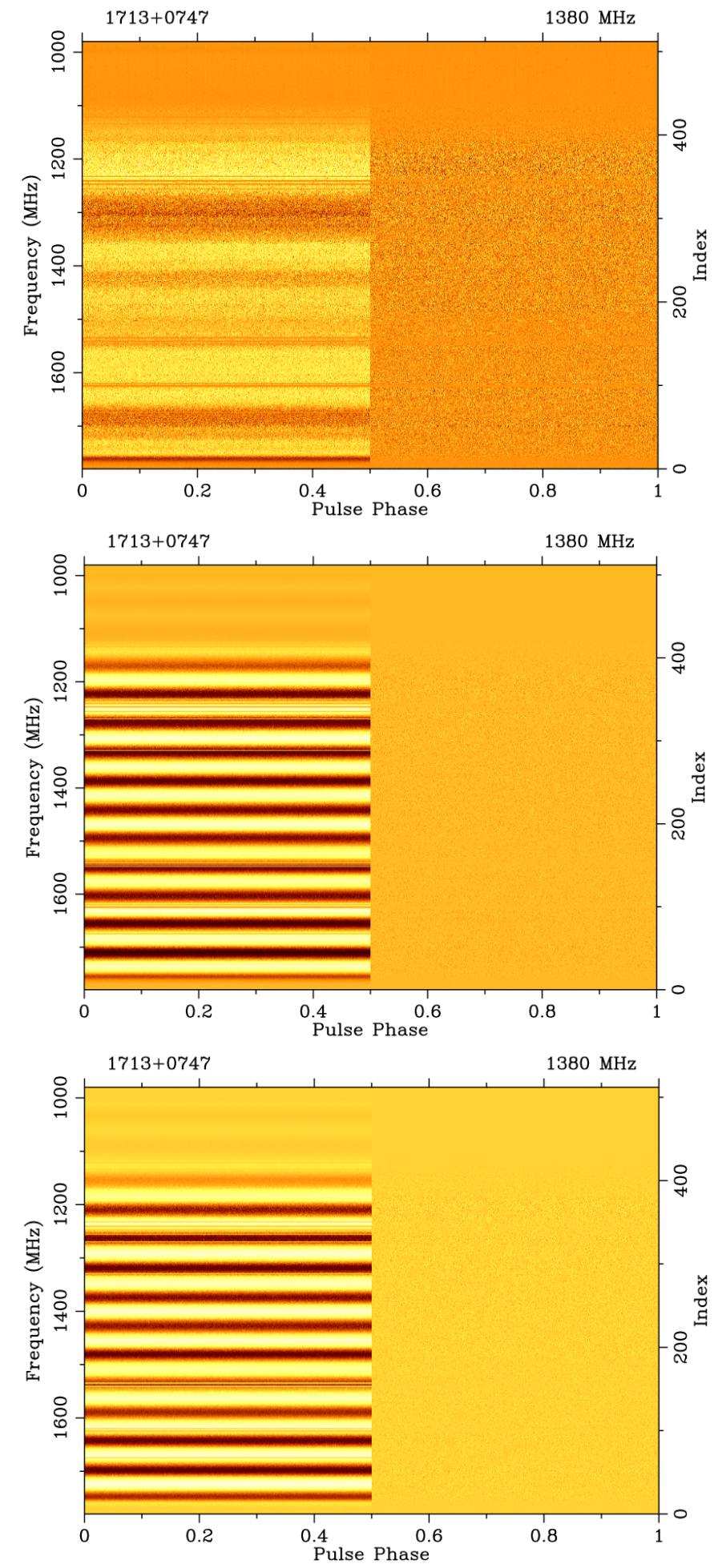

Figure 2.3: Pulse phase vs frequency for a noise diode as seen by the Arecibo Observatory's L-wide receiver. Plotted from top to bottom are the observed Stokes $Q, U$, and $V$ from a 90-second integration of the noise diode signal. Lighter colors correspond to positive intensities, and vice versa. Notice the rotation of the stokes vector about the $Q$ axis on the Poincaré sphere, shown in the Stokes $U$ and $V$ images. 


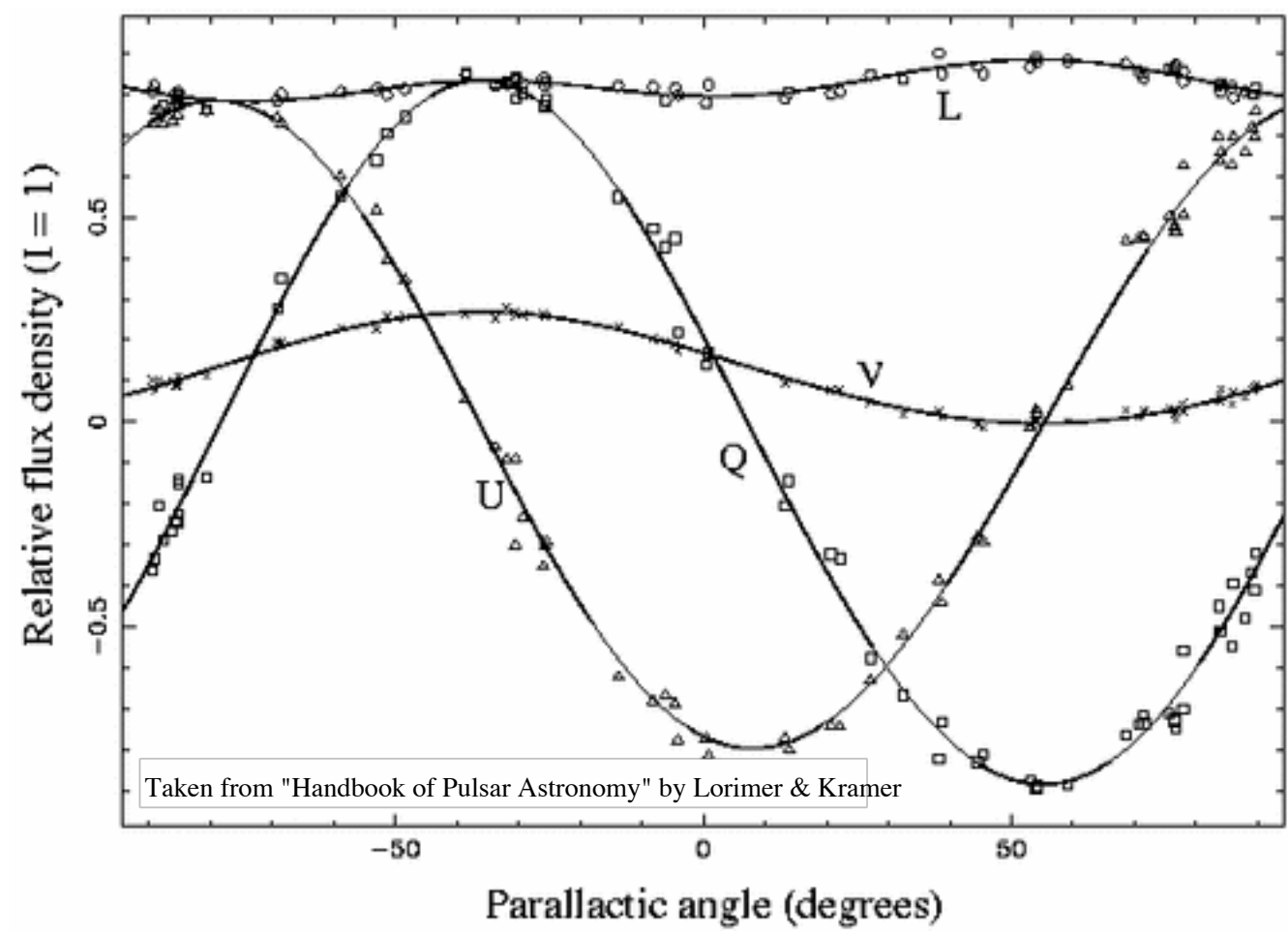

Figure 2.4: The Stokes vectors resulting from observing a polarized source over a wide range of parallactic angles. This data can then be fit to obtain a full Mueller Matrix (see Equation 2.15 and Section 2.1.4). 
band, showing that this receiver (Arecibo's L-Wide receiver) has nonzero differential gain across the band. Further, a side-by-side comparison of the Stokes U and V parameters shows the Stokes $\mathrm{U}$ being converted to Stokes $\mathrm{V}$ and back. This is indicative of a time delay between the two feeds, as a constant time delay translates to a phase delay that varies with frequency. Also important to note is that Stokes U and V oscillate back and forth between positive and negative values. Consequently, if one were to sum the signal over a frequency range that is non-negligible with respect to the bandwidth over which the Stokes Parameters oscillate, the summation will result in a signal that has less polarized emission than was actually emitted.

This effect is known as "bandwidth depolarization", and is a concern not only for perfectly calibrated observations of any source that has intrinsic frequency evolution of its Stokes Parameters, but also for the calibration process itself. The latter is especially true since uncalibrated Stokes Parameters can vary wildly over a given frequency range (as can be seen in Figure 2.3).

\subsubsection{Full Calibration}

To create a receiver solution (that is, a Mueller Matrix that fully describes the receiver), we note that we cannot simply observe a polarized source, determine the measured Stokes Parameters, and solve for the parameters in the Mueller matrix, as combining Equations 2.7 and 2.15 will yield four equations with which we would need to solve for six parameters. We do see, however, that it is possible to track a source for a long time (and therefore over a wide range of parallactic angles, which 
can be precisely determined), and therefore to solve for all parameters in the Mueller matrix.

An example is shown in Figure 2.4, which shows the Stokes Parameters resulting from observing a source over a wide range of parallactic angles, as well as the Stokes Parameters predicted by the best fit receiver parameters. Importantly, since the signals can be affected by the aforementioned bandwidth depolarization, and since the parameters that constitute the Mueller Matrix change with observing frequency (for an example, see the top plot of Figure 2.3, and recall that Stokes $\mathrm{Q}$ is indicative of differential gain, $\gamma$ ), it is important to retain as much frequency resolution as is practicable.

\subsection{Rotating Vector Model}

Now that we have discussed what the Stokes Parameters are and how to accurately measure them by accounting for the spurious effects the receiver causes, we would now like to know what we can actually do with the polarized light we have detected.

One of the most interesting uses for pulsar polarization measurements comes from the source of radio emission outlined in Section 1.2.2.1: namely, that the radio emission is produced by charged particles that originate from the polar cap and flow out from the pulsar along its open magnetic field lines. The curvature radiation produced by this process is emitted tangent to the magnetic field line and polarized parallel to the magnetic field line. The position angle of the linear polarization (see 

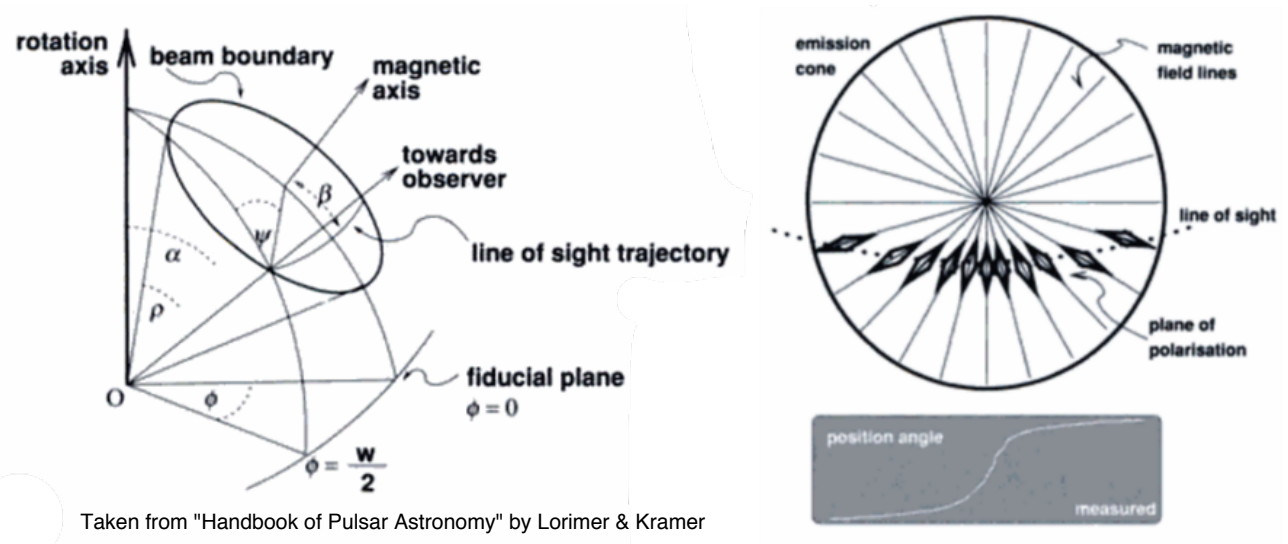

Figure 2.5: Left: Pulsar beam geometry and the pictoral definition of the angles found in Equation 2.17. Right: Variation of the angles of the magnetic field lines (and therefore the angle of polarization due to the curvature radiation produced by them) as the line of sight sweeps through the pulsar's beam. This produces the characteristic "S-shaped" curve to the polarization position angle $\Psi$, and is what is fit by the Rotating Vector Model.

Equation 2.3) is then the same as the angle of the magnetic field lines in the region from which the emission originates.

Therefore, just as the pulse profile represents the pulsar's emission in the part of the magnetosphere being intersected by the line of sight for a given pulse longitude, the "position angle sweep" (that is, the variation of polarization position angle over pulse longitude) represents the direction of the magnetic field intersected by the line of sight for a given pulse longitude. Assuming a perfect dipole, this sweep will vary for different pulsar orientations (see Figure 2.5), allowing an analysis of the position angle sweep to probe the geometry of the pulsar.

This model is known as the Rotating Vector Model (RVM, see Radhakrishnan \& Cooke (1969)) and the equation relating this sweep to the geometrical properties of the pulsar was first proposed by Komesaroff (1970) to be: 


$$
-\tan \left(\Psi-\Psi_{0}\right)=\frac{\sin \alpha \sin \left(\phi-\phi_{0}\right)}{\sin (\alpha+\beta) \cos \alpha+\cos (\alpha+\beta) \sin \alpha \cos \left(\phi-\phi_{0}\right)},
$$

where, just as in Equation 2.3, $\Psi$ is the polarization position angle, $\alpha$ is the angle between the rotation and magnetic axes, $\beta$ is the angle between the magnetic axis and the line of sight, and $\Psi_{0}$ and $\phi_{0}$ are the PA and pulse phase of the inflection point of the PA swing, respectively (see Figure 2.5, left).

While this model proves to be useful in many circumstances, its utility has its limits. First, for pulsars with narrow pulses (such as canonical pulsars), the magnetic field direction is only sampled near the fiducial pulse longitude $\phi_{0}$, yet $\alpha$ and $\beta$ are most easily constrained by determining the magnetic field direction away from $\phi_{0}$. In these circumstances, it can be very difficult (or impossible, practically speaking), to precisely determine $\alpha$ and $\beta$.

Further, some position angle sweeps simply do not follow the phenomenology of the RVM. In some cases, a sudden $90^{\circ}$ jump can be seen in the position angle sweep (see Manchester et al., 1975). This is thought to be due to orthogonally polarized modes present in the emission, and when the dominant mode of emission changes, it produces a sudden jump in the position angle of the linear polarization (see Backer et al., 1975; McKinnon \& Stinebring, 2000). In other cases, non-orthogonal jumps in the position angle sweeps are observed. In still other cases, the position angle sweeps simply do not have shapes that can be fit by the RVM (see PSR B1946+35 in Mitra \& Rankin (2017) or many sources in Johnston \& Weisberg (2006)). 


\subsection{Faraday Rotation}

Setting aside the complexities of the pulsar emission mechanism for a moment, polarimetric observations of pulsars can be used to probe phenomena that are wholly divorced from the pulsar itself. Perhaps the most interesting example of this is through the phenomenon called Faraday rotation.

Before we can detect the polarized light from a pulsar, it must first travel through both the ionized plasma in the interstellar medium, and the Galaxy's magnetic field. To see what effect this will have, let us consider an individual electron as the electromagnetic wave travels past it. First, let us assume the electric field of the pulsar's emission has a typical time dependence of $E(t) \propto e^{-i \omega t}$, and travels in the $\hat{z}$ direction. Further, assume the magnetic field is oriented in the same direction. That is, $\vec{B}=B_{o} \hat{z}$. Then, from the Lorentz force

$$
m_{e} \frac{d \vec{v}}{d t}=-e(\vec{E}+\vec{v} \times \vec{B})
$$

Since $E(t) \propto e^{-i \omega t}, v(t) \propto e^{-i \omega t}$, so

$$
\begin{aligned}
& m_{e} \omega v_{x}=m_{e} \omega^{2} s_{x}=-e\left(E_{x}-i \omega B_{o} s_{y}\right) \\
& m_{e} \omega v_{y}=m_{e} \omega^{2} s_{y}=-e\left(E_{y}+i \omega B_{o} s_{x}\right) .
\end{aligned}
$$

where $s_{x}$ and $s_{y}$ represent displacements in the $x$ and $y$ directions, respectively. Anticipating the desire to talk about right and left circularly polarized waves, we define the electric fields and displacements 


$$
\begin{gathered}
s_{ \pm}=s_{x} \pm i s_{y} \\
E_{ \pm}=E_{x} \pm i E_{y} .
\end{gathered}
$$

Solving Equation 2.19 for $s_{x}$ and $s_{y}$ and substituting into Equation 2.20, we find

$$
s_{ \pm}=\frac{e E_{ \pm}}{m_{e}\left(\omega^{2} \pm \frac{\omega e B_{o}}{m_{e}}\right)} .
$$

We note the cyclotron frequency $\Omega=e B_{o} / m_{e}$, and will henceforth use $\Omega$.

The effect of the light passing through an ionized plasma with a magnetic field in the direction of propagation therefore has the effect of displacing the plasma, creating a total induced dipole moment

$$
P_{ \pm}=n_{e} p_{ \pm}=n_{e} e s_{ \pm}
$$

So

$$
P_{ \pm}=\frac{n_{e} e^{2} E_{ \pm}}{m_{e}\left(\omega^{2} \pm \omega \Omega\right)}
$$

which means the index of refraction

$$
n_{ \pm}=\sqrt{1+4 \pi \frac{P_{ \pm}}{E_{ \pm}}}=\sqrt{1-\frac{4 \pi n_{e} e^{2}}{m_{e} \omega^{2} \pm \omega \Omega}}
$$

Evidently, the right and left circularly polarized waves have different indices of refraction (and therefore different group velocities) as they travel through the plasma. Since the wave number $k_{ \pm}=\frac{n_{ \pm} \omega}{c}$, 


$$
E_{ \pm}=E_{o} e^{\left(\frac{\omega}{c} n_{ \pm} z-\omega t\right)}
$$

We can then use Euler's formula to split these expressions into their real and imaginary components, and combine this with Equation 2.20 to ascribe these components to the contributions of each of these to the $x$ and $y$ components of the total $E$ field. The algebra is left as an exercise for the reader, however we find

$$
\begin{aligned}
& E_{x}=2 E_{o} \cos \left(\frac{\omega}{2 c}\left(n_{+}+n_{-}\right) z-\omega t\right) \cos \left(\frac{\omega}{2 c}\left(n_{+}-n_{-}\right) z\right) \\
& E_{y}=2 E_{o} \cos \left(\frac{\omega}{2 c}\left(n_{+}+n_{-}\right) z-\omega t\right) \sin \left(\frac{\omega}{2 c}\left(n_{+}-n_{-}\right) z\right) .
\end{aligned}
$$

Therefore, the electric field is oriented at an angle

$$
\beta=\tan ^{-1}\left(\frac{E_{x}}{E_{y}}\right)=\frac{\omega}{2 c}\left(n_{+}-n_{-}\right) z .
$$

In reality, the implicit assumption of a constant magnetic field and electron density along the line of sight are not true, however, we can consider them to be functions of $z$ and perform the same analysis for an infinitesimally small distance $d z$, for which we will find the electric field to be rotated by an angle $d \beta$.

Assuming the cyclotron frequency is small compared to the frequency of the emitted light (a reasonable assumption since radio observations are typically performed above $100 \mathrm{MHz}$, while the cyclotron frequency of an electron in a $10 \mu \mathrm{G}$ magnetic field is $<100 \mathrm{~Hz}$ ), we find that

$$
n_{+}-n_{-}=\frac{4 \pi n_{e} e^{2} \Omega}{m_{e} c \omega^{3}},
$$


thus,

$$
d \beta=\frac{2 \pi e^{3}}{m_{e}^{2} c^{2} \omega^{2}} n_{e}(z) B_{\|}(z) d z
$$

where we use $B_{\|}$to stress that the magnetic field is parallel to the line of sight. Using $\omega=\frac{2 \pi c}{\lambda}$ and integrating, we finally see that

$$
\beta=\frac{e^{3} \lambda^{2}}{2 \pi m^{2} c^{4}} \int n_{e}(z) B_{\|}(z) d z
$$

Often times, Equation 2.27 is written in terms of the "Rotation Measure"

$$
\mathrm{RM}=\frac{e^{3}}{2 \pi m^{2} c^{4}} \int n_{e}(z) B_{\|}(z) d z
$$

SO

$$
\beta=\operatorname{RM} \lambda^{2}
$$

Faraday rotation is especially important for pulsars, because basic observations allow for the independent determination of the free electron density along the line of sight, which is called the "dispersion measure"

$$
\mathrm{DM}=\int n_{e} d z
$$

Therefore, if one assumes the magnetic field along the line of sight is constant, it can be pulled out of the integral in Equation 2.28, leaving, 


$$
\mathrm{RM}=B_{\|} \frac{e^{3}}{2 \pi m^{2} c^{4}} \int n_{e}(z) d z
$$

Plugging in values for constants, and using Equation 2.30, we find that a reasonable estimate of the average magnetic field strength along the line of sight to a pulsar is given by

$$
\left\langle B_{\|}\right\rangle=1.23 \frac{\mathrm{RM}}{\mathrm{DM}} \mu \mathrm{G}
$$

All that remains is to measure the RM of a given source! The most apparent way would be to measure the angle of the linear polarization of a pulsar across as wide a range of observing frequencies as possible, and in so doing, measure $\beta$ with respect to $\lambda$. This would then allow a simple fit to be performed to Equation 2.29.

Another method exists to take advantage of bandwidth depolarization. As discussed in Section 2.1.3, if two Stokes vectors are added together, they will partially cancel each other out unless they point in the same direction on the Poincaré sphere. Since uncorrected Faraday rotation will cause such a situation, uncorrected (or miscorrected!) Faraday rotation will serve to reduce the total polarized intensity of a pulsar signal when summed over the observing bandwidth. This effect (bandwidth depolarization due to Faraday rotation) is referred to as "Faraday depolarization". The correct rotation measure, then is that which maximizes the total polarized light when summed over the observing band. 


\section{Chapter 3}

\section{The NANOGrav Nine-Year Data Set and Beyond: Polarimetry and}

\section{Pulse Microcomponents}

Submitted to ApJ with co-authors Maura A. McLaughlin, Paul B. Demorest, Ingrid H. Stairs, Zaven Arzoumanian, Kathryn Crowter, Timothy Dolch, Megan E. DeCesar, Justin A. Ellis, Robert D. Ferdman, Elizabeth C. Ferrara, Emmanuel Fonseca, Marjorie E. Gonzalez, Glenn Jones, Megan L. Jones, Michael T. Lam, Lina Levin, Duncan R. Lorimer, Ryan S. Lynch, Cherry Ng, David J. Nice, Timothy T. Pennucci, Scott M. Ransom, Paul S. Ray, Ren'ee Spiewak, Kevin Stovall, Joseph K. Swiggum, and Weiwei Zhu

In this chapter, we present the polarization profiles of the pulsars observed at the Arecibo Observatory (AO) as part of NANOGrav's pulsar timing campaign. We also present the implementation of a calibration technique described in van Straten (2013), whereby PRs are created quickly, allowing them to be made on an epoch-by-epoch basis. In section 3.2 and 3.2.1, we describe this technique.

\subsection{Observations}

The NANOGrav Collaboration et al. (2015) describes the full details of NANOGrav's data collection process, and we summarize the relevant parts of that process here. Note that we only used data collected at the Arecibo Observatory with the wideband 
$\mathrm{PUPPI}^{1}$ instrument.

Sources were observed spanning MJDs 56989 to 56874, with a quasi-monthly cadence with AO's S-Wide (center frequency 2.1 GHz), L-Wide (center frequency $1.4 \mathrm{GHz}$ ) and $430 \mathrm{MHz}$ receivers (or some combination of the three that was chosen to most efficiently capitalize on each individual pulsar's multi-frequency characteristics). The typical integration time of a single observation was approximately 20 minutes. At 2.1 and $1.4 \mathrm{GHz}$ (both dual linear feeds), the available bandwidth is 800 $\mathrm{MHz}$, which is split into 512 frequency channels, while at $430 \mathrm{MHz}$ (a dual circular feed), the available bandwidth is $100 \mathrm{MHz}$ and is split into 64 channels.

At all observing frequencies, the data were folded and coherently dedispersed in real time. Then, frequency channels affected by RFI were excised and flux calibrator observations were used to convert telescope intensity into flux. Reported fluxes were determined by averaging fluxes over all epochs.

\subsection{Data Reduction}

\subsubsection{Polarimetric Calibration}

\subsubsection{Measurement Equation Modeling}

To estimate the absolute gain, differential gain, and the phase offset of the receiver feeds, a broadband, pulsed signal was injected at $45^{\circ}$ to both receiver feeds. This signal was observed before each observation for $\sim 90$ seconds and RFI was then excised from these observations as well. This procedure is part of the standard

\footnotetext{
${ }^{1}$ Puerto Rican Ultimate Pulsar Processing Instrument
} 
NANOGrav data reduction process.

To estimate the magnitude of the cross-coupling between the receiver feeds, observations of PSR J0528+2200 (PSR B0525+21), a bright, strongly polarized source, were taken over a wide range of parallactic angles. To maximize the efficiency of the observing time, the observing frequency was switched between $430 \mathrm{MHz}, 1.4$ $\mathrm{GHz}$, and 2.1 GHz. Each time the observing frequency was changed, we injected a pulsed noise signal. This process yielded polarimetric data for each of the observing frequencies for which we have corresponding NANOGrav pulsar data, allowing us to extract three full polarimetric responses (PRs) (as described in van Straten, 2004) with one observation.

These responses were then used to correct for the cross-coupling of the receiver feeds. After using these responses to calibrate our data, we then compared the resulting profiles of PSR B1937+21 and PSR J1713+0474 to previously published profiles. These sources were chosen for comparison because they had a high signalto-noise ratio (SNR) and many consistent published polarization profiles.

Since the polarization profiles generated by this method were not consistent epoch-to-epoch, we used PSRs J1713+0747 and B1937+21 as "standard sources" in the Measurement Equation Template Matching (METM) method, described in sections 3.1 and 5 of van Straten (2013) by choosing profiles for these sources that appeared to be consistent with previously published profiles. This allowed us to use subsequent observations of these sources to generate a new PR. In reality, since the observations being used to generate the METM PRs had already been calibrated with the MEM-generated PR, the METM-generated PRs should be thought of as 
Table 1: Total Observation Lengths and Derived Rotation Measures ${ }^{\text {a }}$

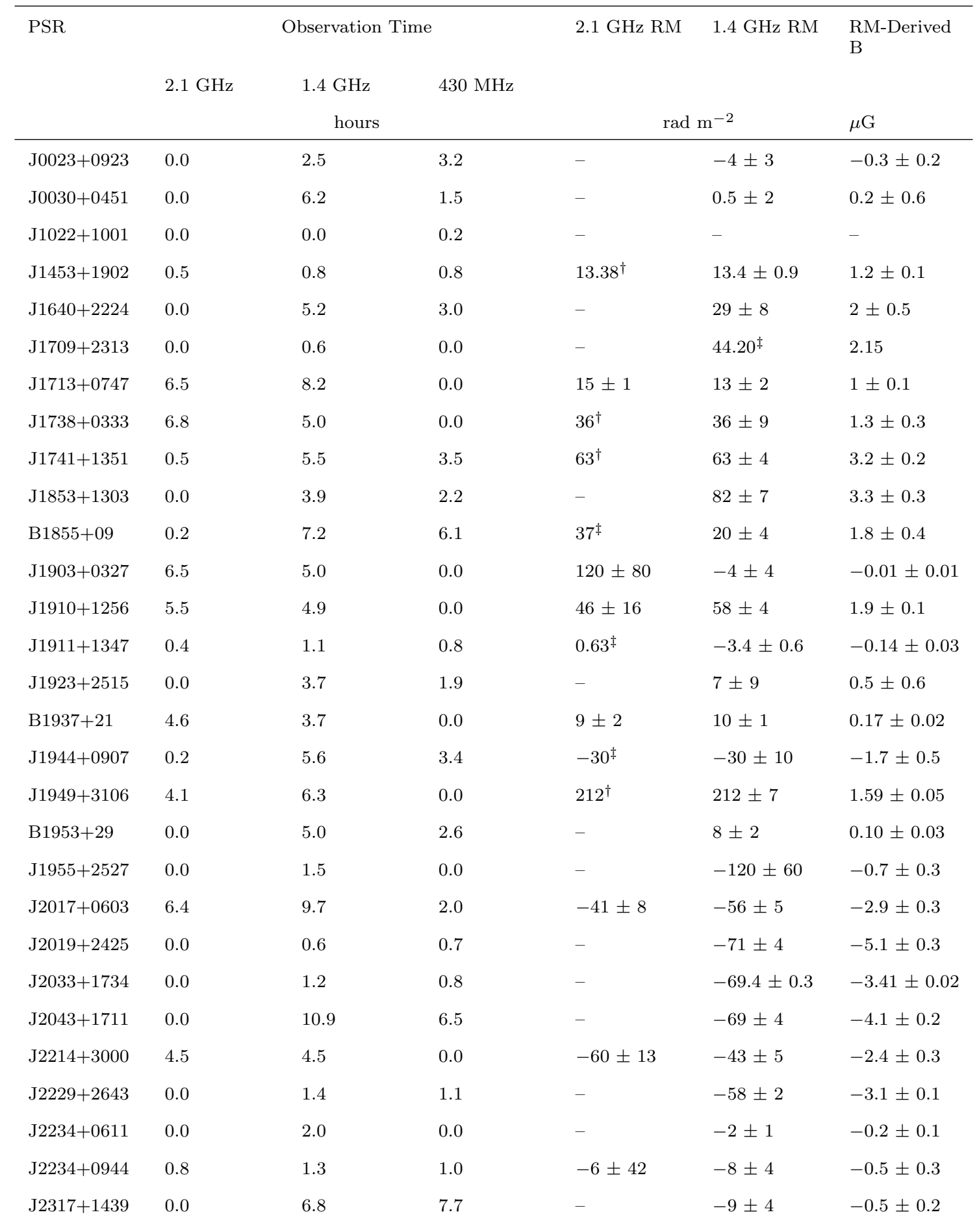

${ }^{a}$ Errors on RM represent the standard epoch to epoch deviation of RM. Values with a ${ }^{\dagger}$ have no error because the data were too low SNR, so the average $1.4 \mathrm{GHz}$ RM was used. Values with a $\ddagger$ have no reported error because there was only one observation at this frequency. 
per-epoch corrections to the MEM-generated PR.

Next, having already calibrated all of the observations in NANOGrav's AO data set with the MEM-generated PR, we were able to apply these corrections to these observations by selecting the PR correction whose epoch is closest to the epoch of the observation that is to be calibrated.

\subsubsection{Faraday Rotation Correction}

As described above, one of the consequences of the propagation of polarized light through the interstellar medium is the rotation of the angle of the linearly polarized emission, known as Faraday rotation. To correct for this effect, we follow Han et al. (2006) and others and find the RM at which the total observed linear polarization is maximized.

We found that we could reliably fit an RM value to data from most $1.4 \mathrm{GHz}$ observations and some $2.1 \mathrm{GHz}$ observations. For data in which RM could not be

fit (430 MHz observations and some 2.1 GHz observations), we used the RM from 1.4 GHz to correct for Faraday rotation.

\subsection{Results}

Here, we present the profiles resulting from the calibration scheme described in Section 3.2. For each source at each observing frequency, we describe the time and frequency-averaged polarization profile and compare it to both our own results at other frequencies and to previously published results, if any exist. Profiles are 
Table 2: Total Intensity Emission Parameters

\begin{tabular}{|c|c|c|c|c|c|c|c|}
\hline \multirow[t]{2}{*}{ PSR } & \multicolumn{3}{|c|}{ Flux Density } & \multicolumn{3}{|c|}{ Duty Cycle } & \multirow{2}{*}{$\begin{array}{l}\text { Spectral } \\
\text { Index }\end{array}$} \\
\hline & $\begin{array}{l}2.1 \mathrm{GHz} \\
\mathrm{mJy}\end{array}$ & $\begin{array}{l}1.4 \mathrm{GHz} \\
\mathrm{mJy}\end{array}$ & $\begin{array}{l}430 \mathrm{MHz} \\
\mathrm{mJy}\end{array}$ & $2.1 \mathrm{GHz}$ & $1.4 \mathrm{GHz}$ & $430 \mathrm{MHz}$ & \\
\hline J0023+0923 & - & 0.4 & 6.0 & - & 0.30 & 0.30 & -2.2 \\
\hline J0030+0451 & - & 1.2 & 15.0 & - & 0.54 & 0.60 & -2.1 \\
\hline $\mathrm{J} 1022+1001$ & - & - & 11.0 & - & - & 0.17 & - \\
\hline $\mathrm{J} 1453+1902$ & 0.05 & 0.1 & 1.8 & 0.15 & 0.29 & 0.50 & -2.1 \\
\hline $\mathrm{J} 1640+2224$ & - & 0.7 & 18.9 & - & 0.25 & 0.28 & -2.8 \\
\hline $\mathrm{J} 1709+2313$ & - & 0.1 & - & - & 0.34 & - & - \\
\hline $\mathrm{J} 1713+0747$ & 6.2 & 11.6 & - & 0.77 & 0.89 & - & -1.2 \\
\hline $\mathrm{J} 1738+0333$ & 0.6 & 0.7 & - & 0.36 & 0.37 & - & -0.3 \\
\hline $\mathrm{J} 1741+1351$ & 0.03 & 0.5 & 4.3 & 0.04 & 0.38 & 0.41 & -2.7 \\
\hline $\mathrm{J} 1853+1303$ & - & 0.5 & 7.3 & - & 0.55 & 0.38 & -2.2 \\
\hline $\mathrm{B} 1855+09$ & 3.5 & 3.8 & 18.4 & 0.38 & 0.75 & 0.71 & -1.0 \\
\hline $\mathrm{J} 1903+0327$ & 0.7 & 0.8 & - & 0.22 & 0.63 & - & -0.1 \\
\hline $\mathrm{J} 1910+1256$ & 0.3 & 0.5 & - & 0.18 & 0.31 & - & -1.0 \\
\hline $\mathrm{J} 1911+1347$ & 1.0 & 1.0 & 2.2 & 0.54 & 0.53 & 0.46 & -0.5 \\
\hline $\mathrm{J} 1923+2515$ & - & 0.3 & 4.2 & - & 0.59 & 0.32 & -2.4 \\
\hline B1937+21 & 7.1 & 13.4 & - & 0.82 & 0.84 & - & -1.2 \\
\hline $\mathrm{J} 1944+0907$ & 0.7 & 2.8 & 33.0 & 0.59 & 0.75 & 0.74 & -2.3 \\
\hline $\mathrm{J} 1949+3106$ & 0.07 & 0.1 & - & 0.07 & 0.10 & - & -1.2 \\
\hline $\mathrm{B} 1953+29$ & - & 0.9 & 27.2 & - & 0.78 & 0.50 & -2.9 \\
\hline $\mathrm{J} 1955+2527$ & - & 0.4 & - & - & 0.26 & - & - \\
\hline $\mathrm{J} 2017+0603$ & 0.3 & 0.4 & 2.2 & 0.35 & 0.62 & 0.54 & -1.2 \\
\hline $\mathrm{J} 2019+2425$ & - & 0.3 & 6.3 & - & 0.48 & 0.35 & -2.6 \\
\hline $\mathrm{J} 2033+1734$ & - & 0.4 & 2.9 & - & 0.45 & 0.45 & -1.6 \\
\hline $\mathrm{J} 2043+1711$ & - & 0.2 & 4.4 & - & 0.82 & 0.41 & -2.6 \\
\hline $\mathrm{J} 2214+3000$ & 0.9 & 0.6 & - & 0.47 & 0.60 & - & 0.6 \\
\hline $\mathrm{J} 2229+2643$ & - & 0.7 & 5.7 & - & 0.27 & 0.28 & -1.8 \\
\hline $\mathrm{J} 2234+0611$ & - & 1.3 & - & - & 0.55 & - & - \\
\hline $\mathrm{J} 2234+0944$ & 1.2 & 2.1 & 3.4 & 0.50 & 0.82 & 0.45 & -0.6 \\
\hline $\mathrm{J} 2317+1439$ & - & 0.8 & 46.4 & - & 0.45 & 0.23 & -3.5 \\
\hline
\end{tabular}


shown in Appendix A. We note that epoch to epoch DM variations will introduce some dispersive smearing, for all sources, the timescale for this smearing is $<1 \%$ of the pulsar's spin period given the level of variation shown in Jones et al. (2017), and is therefore negligible. The parameters describing the observations themselves (such as observation times and frequencies) and derived RMs are summarized in Table 3.1. Parameters describing the total intensity emission from each of the sources in this data set are shown in Table 3.2, where we have defined "duty cycle" as the ratio of bins where the total intensity emission was above the baseline (as determined by eye) to the total number of bins, rather than the definition found in Lorimer \& Kramer (2005), and the spectral index was found by averaging the total flux over all epochs for each frequency band. Polarization emission fractions for each source in this data set are shown in Table 3.3 where angle brackets denote a phase-averaged quantity, and bars denote an absolute value.

Also, we note the presence of instrumental effects in the data. For example, the linearly polarized emission for some sources exceeds the total intensity emission in some phase bins. In some instances, this is caused by the linear polarization noise being biased by virtue of it being a quantity derived from a quadrature sum, while in other cases, it is due to baselining effects. In these latter cases, the linearly polarized emission in said phase bins should be viewed as an aberration. Additionally, for certain bright sources, sampling effects cause the pulsar signal to be aliased back into the band. Therefore, some of the low-intensity emission seen in the profiles presented here is not intrinsic, but rather due to instrumental effects (see Pennucci, 2015, for more details on this issue). We stress, though, that while this effect 
accounts for some of the low-intensity emission, we do see additional low-intensity emission that is not due to this effect and is therefore intrinsic to the source itself.

\subsubsection{PSR J0023+0923}

\subsubsection{1.4 GHz}

Not much polarization data have been published for PSR J0023+0923, however what has been published (Craig, 2014), shows many similarities to our results in that the total intensity profile shows a leading component that has a relatively low intensity followed by two relatively high intensity components. The linear intensity profile also shows three components as well as evidence for a bridge of linearly polarized emission connecting them all. Although the leading component again has a lower intensity than the trailing two components, the disparity is not as pronounced as in the total intensity profile. Also of note is that the final component in the linear polarization profile is noticeably narrower than the component immediately preceding it, whereas the widths of their total intensity counterparts are not as disparate.

For all the similarities our results show, there are some differences. First, the circular polarization profile shows a left circularly polarized (LCP) peak that coincides with the final total intensity component, as well as evidence for a right circularly polarized (RCP) peak immediately preceding it. There is also evidence for an LCP peak coinciding roughly with the middle total intensity component, and a RCP peak coinciding with the leading total intensity component. It appears as 
though this would be consistent with Craig (2014) if they had also shown negative circular polarization intensities, modulo an overall negative sign. This suggests that they are using the IAU convention for circular polarization, although the circular polarization sign convention used in the paper is not stated. In addition, the total intensity profile they present shows that the final component has a significantly higher intensity than the component immediately preceding it. As the relative intensity of these components changes substantially with frequency, it is likely that the pulsar was detected much more strongly in the lower part of the observing bandwidth (where the final component is strongest) during the observations taken for the analysis presented in Craig (2014).

\subsubsection{2 $430 \mathrm{MHz}$}

No polarization profiles have been published for PSR J0023+0923 at $430 \mathrm{MHz}$, however Bangale et al. (in prep) presents the total intensity profile as seen by the $350 \mathrm{MHz}$ receiver on the Green Bank Telescope, whose frequency range overlaps with the AO $430 \mathrm{MHz}$ receiver. Both profiles show a very narrow pulse, however what appears to be a single component profile in the published Green Bank Telescope profile is shown to be a multi-component main pulse consisting of an extremely bright component preceded by a low-intensity peak and followed by a moderateintensity ridge. This main pulse is preceded by a low-intensity pulse that does not appear to be connected to the main pulse and is aligned with the precursor seen at $1.4 \mathrm{GHz}$. 
The profile is not strongly polarized, with the only significant features being a peak in both the linear polarization and the right-circular polarization coincident with the total intensity peak.

\subsubsection{PSR J0030+0451}

\subsubsection{1 $1.4 \mathrm{GHz}$}

PSR J0030+0451 has a complex profile at $1.4 \mathrm{GHz}$, drawing interest even upon discovery (Lommen et al., 2000). Their analysis found a total intensity profile consisting of at least six components. Our results largely agree with this analysis, however the significant increase in signal-to-noise provided by this work reveals that the interpulse may be more complex than the two component model described in Lommen et al. (2000). We also do not see the "bump" situated between the main pulse and the interpulse (corresponding to a rotational phase of $\sim 0.8$ in our analysis), although both components appear to be wider than originally reported.

The linear polarization profile of PSR J0030+0451 is similar in shape to that of the total intensity profile, although the main pulse shows two low-intensity linear polarization peaks at the leading edge of the main pulse and another low-intensity linear polarization peak on the trailing edge of the main pulse. The interpulse shows three distinct components that coincide with three components of the total intensity profile.

The circular polarization profile shows a strong LCP peak coinciding with the total intensity peak, along with lower-intensity peaks in RCP and LCP following it, 
The interpulse shows almost no circular polarization, although there is evidence for a wide, RCP pulse spanning almost the entire interpulse width.

\subsubsection{2 $430 \mathrm{MHz}$}

The polarization profile for PSR J0030+0451 at $430 \mathrm{MHz}$ is in good agreement with that presented in Lommen et al. (2000), however there are small differences. As previously reported, the linear polarization profile of the main pulse has many components, with the overall peak coinciding with the total intensity peak, however our data do not show the linear polarization intensity dropping off as steeply afterwards. The circular polarization profile is also largely in agreement with that shown in Lommen et al. (2000), with the only significant difference being the RCP peak presented in this work is more significant.

\subsubsection{PSR J1022+1001}

\subsubsection{1 $430 \mathrm{MHz}$}

PSR J1022+1001 profile has proven to be variable, most notably with the respective intensities of the two components that make up the total intensity profile. In this work, we see the leading component have a higher intensity than the trailing component, consistent with some later observations (Stairs et al. (1999), for example), but not with its discovery Camilo et al. (1996a). The linearly polarized emission also shows two primary components, the first of which slightly precedes its total intensity counterpart, while the other is aligned with its total intensity 
counterpart. The circularly polarized emission is entirely LCP and also shows two components, although unlike the linearly polarized emission, these components are aligned with their total intensity counterparts.

\subsubsection{PSR J1453+1902}

\subsubsection{2.1 GHz}

The SNR of the $2.1 \mathrm{GHz}$ profile for PSR J1453+1902 is not very high, but one component is clearly detected. That component shows moderate linear polarization, which also consists of one component, the center of which precedes the main total intensity component. We do not detect any circularly polarized emission.

\subsubsection{2 $1.4 \mathrm{GHz}$}

Much of PSR J1453+1902's total intensity profile at 1.4 GHz resembles its 2.1 $\mathrm{GHz}$ counterpart, as the main part of the profile consists of one bright component. However, the higher SNR at $1.4 \mathrm{GHz}$ reveals an additional component leading the brightest component. As with the $2.1 \mathrm{GHz}$ profile, there is a bright linearly polarized peak of emission that slightly precedes the total intensity peak, although it appears as though there is another, very weak component trailing that. The new total intensity component is $100 \%$ polarized. As with the $2.1 \mathrm{GHz}$ profile, we do not detect significant circular polarization. 


\subsubsection{3 $430 \mathrm{MHz}$}

The only published profile for PSR J1453+1902 is a $430 \mathrm{MHz}$ total intensity profile that agrees well with our $430 \mathrm{MHz}$ profile (Lorimer et al., 2007), showing a leading, low intensity component, followed by much stronger component that has a faint, trailing pulse attached to it. The flux density of the total intensity profile is also in agreement with the analysis done in Lorimer et al. (2007). The linear polarization profile has changed markedly from the 2.4 and $1.4 \mathrm{GHz}$ linear intensity profiles, showing one, low intensity component that appears to be aligned with the total intensity peak. The circularly polarized profile is also very different at 430 $\mathrm{MHz}$, where there is a clear LCP peak aligned with the total intensity peak, whereas at higher frequencies, there is no detectable circular polarization.

\subsubsection{PSR J1640+2224}

\subsubsection{1 $1.4 \mathrm{GHz}$}

PSR J1640+2224's pulse profile at $1.4 \mathrm{GHz}$ is a single pulse comprised of at least three components (Kramer et al., 1998). The linear polarization profile shows four distinct components, the brightest of which coincides with the total intensity peak. PSR J1640+2224 also shows a strong LCP peak (with a higher intensity than the linear polarization peak) coincident with the total intensity peak, and flanked by low-intensity RCP emission. 


\subsubsection{2 $430 \mathrm{MHz}$}

PSR J1640+2224's pulse profile at $430 \mathrm{MHz}$ is similar to the profile at $1.4 \mathrm{GHz}$, as the components on the $1.4 \mathrm{GHz}$ profile are all still present and the total profile widths are very similar (Foster et al., 1995). The linear polarization profile shows two main components that coincide with two components of the total intensity profile, and a trailing linear polarization tail. The circular polarization is wholly RCP and is nearly identical to the linear polarization profile, although slightly of slightly lower intensity.

\subsubsection{PSR J1709+2313}

\subsubsection{1 $1.4 \mathrm{GHz}$}

The $1.4 \mathrm{GHz}$ profile of PSR J1709+2313 presented here is in agreement with the previously published profile (Lewandowski et al., 2004), and includes a main pulse with at least 3 components in addition to an interpulse. The interpulse is almost entirely linearly polarized, as is the leading component of the main pulse. There is no detectable circular polarization.

\subsubsection{PSR J1713+0747}

\subsubsection{1 $2.1 \mathrm{GHz}$}

The pulse profile for PSR J1713+0747 at $2.1 \mathrm{GHz}$ has been studied before (Dai et al., 2015), and our observations confirm that analysis, however, the increased sen- 
sitivity of our data set allows us to report previously undetected microcomponents, as we are able to detect small components preceding and trailing the previously published components. These microcomponents are 50 to 100 times less intense than the total intensity peak, respectively, yet almost doubles the width of the pulse. The leading component shows slight linear polarization, yet because of its relatively low total intensity, this mall level of polarization means that the leading component is significantly polarized. The trailing component, however, does not show much polarization.

\subsubsection{2 $1.4 \mathrm{GHz}$}

PSR J1713+0747 has been studied extensively at $1.4 \mathrm{GHz}$, and in terms of the most intense components, we again find agreement with the existing literature (see, for example Dai et al. (2015); Ord et al. (2004); Yan et al. (2011), and more). Yet as with the $2.1 \mathrm{GHz}$ data, we find substantial structure with intensities $\sim 100$ times smaller than the peak total intensity. Indeed, we see the same microcomponents that were evident in the $2.1 \mathrm{GHz}$ emission, although they are much higher SNR at 1.4 GHz. Also, what appeared to be a short tail preceding the first total intensity microcomponent appears here to be a much longer tail, spanning nearly the entire rotation of the pulsar. 


\subsubsection{PSR J1738+0333}

\subsubsection{1 $2.1 \mathrm{GHz}$}

The $2.1 \mathrm{GHz}$ total intensity profile for PSR J1738+0333 consists of one main pulse made up of two strong components preceded by two low-intensity components. The linear polarization profile shows many similarities to the total intensity profile, having 3 distinct components that correspond to components of the total intensity profile. The circular polarization profile has one strong component, and one weakly detected component. Both of these components are RCP and correspond to the strong total intensity components.

\subsubsection{2 $1.4 \mathrm{GHz}$}

The 1.4 GHz profile for PSR J1738+0333 presented in this work looks very similar not only to previously published profiles (Jacoby, 2005; Freire et al., 2012), but also to its $2.1 \mathrm{GHz}$ profile. The linear polarization profile has 3 distinct components that have corresponding components in the total intensity profile and the circular polarization profile consists of two components of all RCP emission that coincide to the strongest components of the total intensity profile. Indeed, the only new feature that was not seen in the $2.1 \mathrm{GHz}$ data is a very weak component on the trailing edge of the main pulse. 


\subsubsection{PSR J1741+1351}

\subsubsection{2.1 GHz}

The $2.1 \mathrm{GHz}$ profile for PSR J1741+1351 is relatively simple: one bright, single-component pulse. There does seem to be another component preceding the main pulse by 0.3 rotations, but this component is very weak, and therefore cannot be characterized further. The polarization profile for PSR J1741+1351 at $2.1 \mathrm{GHz}$ contains no detectable polarized emission.

\subsubsection{2 $1.4 \mathrm{GHz}$}

The $1.4 \mathrm{GHz}$ profile for PSR J1741+1351 shows the low-intensity component seen in the $2.1 \mathrm{GHz}$ in much greater detail. In addition, the bright, single-component pulse seen at $2.1 \mathrm{GHz}$ is not seen to consist of one bright component preceded by a low-intensity "bump" and followed by a long, low-intensity tail. This tail was not reported in previously published studies (Jacoby et al., 2007; Espinoza et al., 2013), The linearly polarized intensity profile shows that the leading part of the profile's linearly polarized emission also has two components that coincide with the two components of the total intensity emission. The trailing part of the linearly polarized intensity profile has three clear components, two of which coincide with components of the total intensity profile. There is no detectable linear polarization that coincides with the low-intensity tail seen in the total intensity profile. The circular polarization profile shows a LCP pulse corresponding to the leading part of the total intensity profile, followed by a multi-component section with many changes 
in senses of circular polarization. 
Table 3: Polarized Intensity Parameters

\begin{tabular}{|c|c|c|c|c|c|c|c|c|c|c|c|c|}
\hline \multirow[t]{2}{*}{ PSR } & \multicolumn{3}{|c|}{$\langle P\rangle / I$} & \multicolumn{3}{|c|}{$\langle L\rangle / I$} & \multicolumn{3}{|c|}{$\langle V\rangle / I$} & \multicolumn{3}{|c|}{$\langle|V|\rangle / I$} \\
\hline & $2.1 \mathrm{GHz}$ & $1.4 \mathrm{GHz}$ & $430 \mathrm{MHz}$ & $2.1 \mathrm{GHz}$ & $1.4 \mathrm{GHz}$ & $\begin{array}{c}430 \mathrm{MHz} \\
\text { Percent }\end{array}$ & $2.1 \mathrm{GHz}$ & $1.4 \mathrm{GHz}$ & $430 \mathrm{MHz}$ & $2.1 \mathrm{GHz}$ & $1.4 \mathrm{GHz}$ & $430 \mathrm{MHz}$ \\
\hline J0023+0923 & - & 0.30 & 0.34 & - & 0.29 & 0.27 & - & 0.04 & -0.05 & - & 0.06 & 0.17 \\
\hline J0030+0451 & - & 0.33 & 0.25 & - & 0.33 & 0.22 & - & 0.01 & -0.04 & - & 0.04 & 0.08 \\
\hline $\mathrm{J} 1022+1001$ & - & - & 0.47 & - & - & 0.35 & - & - & 0.22 & - & - & 0.26 \\
\hline $\mathrm{J} 1453+1902$ & 1.12 & 0.81 & 0.74 & 0.94 & 0.72 & 0.55 & 0.04 & -0.04 & 0.24 & 0.49 & 0.26 & 0.40 \\
\hline $\mathrm{J} 1640+2224$ & - & 0.15 & 0.23 & - & 0.13 & 0.19 & - & 0.02 & -0.10 & - & 0.08 & 0.11 \\
\hline $\mathrm{J} 1709+2313$ & - & 0.57 & - & - & 0.49 & - & - & 0.03 & - & - & 0.23 & - \\
\hline $\mathrm{J} 1713+0747$ & 0.32 & 0.33 & - & 0.31 & 0.32 & - & -0.02 & -0.01 & - & 0.03 & 0.03 & - \\
\hline $\mathrm{J} 1738+0333$ & 0.29 & 0.22 & - & 0.25 & 0.22 & - & -0.05 & -0.02 & - & 0.12 & 0.04 & - \\
\hline $\mathrm{J} 1741+1351$ & 0.55 & 0.22 & 0.22 & 0.47 & 0.21 & 0.15 & -0.06 & 0.01 & -0.06 & 0.23 & 0.05 & 0.13 \\
\hline $\mathrm{J} 1853+1303$ & - & 0.33 & 0.33 & - & 0.24 & 0.25 & - & 0.03 & -0.10 & - & 0.18 & 0.18 \\
\hline B1855+09 & 0.22 & 0.15 & 0.16 & 0.19 & 0.13 & 0.13 & -0.03 & -0.01 & -0.03 & 0.09 & 0.05 & 0.08 \\
\hline $\mathrm{J} 1903+0327$ & 0.22 & 0.21 & - & 0.16 & 0.18 & - & -0.08 & -0.06 & - & 0.13 & 0.08 & - \\
\hline $\mathrm{J} 1910+1256$ & 0.26 & 0.24 & - & 0.20 & 0.19 & - & 0.02 & -0.00 & - & 0.15 & 0.14 & - \\
\hline $\mathrm{J} 1911+1347$ & 0.52 & 0.43 & 0.80 & 0.42 & 0.34 & 0.62 & 0.14 & 0.18 & -0.02 & 0.26 & 0.23 & 0.41 \\
\hline $\mathrm{J} 1923+2515$ & - & 0.33 & 0.29 & - & 0.29 & 0.21 & - & -0.03 & -0.13 & - & 0.11 & 0.18 \\
\hline $\mathrm{B} 1937+21$ & 0.30 & 0.32 & - & 0.29 & 0.31 & - & -0.01 & 0.00 & - & 0.04 & 0.02 & - \\
\hline $\mathrm{J} 1944+0907$ & 1.29 & 0.16 & 0.20 & 1.04 & 0.12 & 0.11 & 0.02 & 0.07 & -0.12 & 0.61 & 0.08 & 0.14 \\
\hline $\mathrm{J} 1949+3106$ & 0.30 & 0.17 & - & 0.26 & 0.15 & - & -0.02 & 0.01 & - & 0.12 & 0.06 & - \\
\hline B1953+29 & - & 0.34 & 0.19 & - & 0.23 & 0.15 & - & -0.08 & -0.03 & - & 0.23 & 0.10 \\
\hline $\mathrm{J} 1955+2527$ & - & 0.15 & - & - & 0.10 & - & - & -0.07 & - & - & 0.10 & - \\
\hline $\mathrm{J} 2017+0603$ & 0.59 & 0.38 & 0.69 & 0.49 & 0.37 & 0.54 & 0.00 & -0.02 & -0.03 & 0.25 & 0.06 & 0.35 \\
\hline $\mathrm{J} 2019+2425$ & - & 0.49 & 0.39 & - & 0.43 & 0.29 & - & 0.00 & 0.09 & - & 0.17 & 0.22 \\
\hline J2033+1734 & - & 0.42 & 0.38 & - & 0.37 & 0.28 & - & -0.02 & -0.06 & - & 0.14 & 0.21 \\
\hline $\mathrm{J} 2043+1711$ & - & 0.61 & 0.46 & - & 0.57 & 0.30 & - & 0.03 & -0.22 & - & 0.12 & 0.31 \\
\hline $\mathrm{J} 2214+3000$ & 0.36 & 0.38 & - & 0.32 & 0.38 & - & -0.02 & 0.00 & - & 0.11 & 0.03 & - \\
\hline $\mathrm{J} 2229+2643$ & - & 0.24 & 0.40 & - & 0.22 & 0.30 & - & 0.03 & 0.09 & - & 0.07 & 0.21 \\
\hline $\mathrm{J} 2234+0611$ & - & 0.32 & - & - & 0.31 & - & - & 0.03 & - & - & 0.05 & - \\
\hline $\mathrm{J} 2234+0944$ & 0.24 & 0.20 & 0.71 & 0.19 & 0.17 & 0.57 & 0.07 & 0.07 & -0.01 & 0.13 & 0.08 & 0.34 \\
\hline $\mathrm{J} 2317+1439$ & - & 0.29 & 0.14 & - & 0.26 & 0.10 & - & 0.06 & -0.04 & - & 0.07 & 0.08 \\
\hline
\end{tabular}




\subsubsection{3 $430 \mathrm{MHz}$}

As expected (Espinoza et al., 2013), PSR J1741+1351's 430 MHz total intensity profile profile looks similar to the $1.4 \mathrm{GHz}$ profile, however, the components immediately leading and following the strongest component are different. The former is much stronger relative to the total intensity peak, whereas the latter (seen at $1.4 \mathrm{GHz}$ as a long tail) is seen as two distinct components. The linear polarization profile has changed significantly. Indeed, there is almost no detectable linearly polarized emission, save a small peak of emission coinciding with the total intensity peak. The circular polarization profile shows two RCP components, one coinciding with the total intensity peak, and one coinciding with the total intensity component preceding it.

\subsubsection{PSR J1853+1303}

\subsubsection{1 $1.4 \mathrm{GHz}$}

The overall shape of PSR J1853+1303 is quite complex, and spans more than half of the rotation of the pulsar (Gonzalez et al., 2011; Stairs et al., 2005). This work shows a clear bridge of emission spanning the two major components of the profile. In light of this complexity, it is no surprise that the linear polarization profile shows significant complexity as well, containing six distinct components. Interestingly, only two of them seem to coincide with components found in the total intensity profile. Further, the two components that do have corresponding components of the total intensity profile are of relatively low intensity. The circular polarization 
profile, on the other hand, contains a strong LCP peak flanked by two strong RCP peaks, and they all appear to coincide with total intensity components.

\subsubsection{2 $430 \mathrm{MHz}$}

The $430 \mathrm{MHz}$ profile for PSR J1853+1303 shows similar complexity to the 1.4 GHz profile, however some differences are apparent. For example, the relative brightnesses of the three brightest components have changed, and the bridge of emission connecting the two main parts of the pulse profile is no longer detectable above the noise. For as much as the total intensity profile has changed, though, the linear polarization profile has changed wholly. One broad pulse spans the first part of the profile, and there is no detectable linear intensity emission coincident with the second park of the pulse profile. The circular polarization profile has undergone a similar transformation: the strong LCP pulse seen at $1.4 \mathrm{GHz}$ has turned into a strong RCP pulse, and the RCP pulse following it has turned into a weak LCP

pulse. In addition, some weak circularly polarized emission is coincident with the trailing part of the profile.

\subsubsection{PSR B1855+09}

\subsubsection{1 $1.4 \mathrm{GHz}$}

The $1.4 \mathrm{GHz}$ pulse profile has been well studied (Kramer et al. (1998), Yan et al. (2011), Dai et al. (2015), and many more) and shows a total intensity profile that is consistent with our work, however, we clearly detect a bridge of emission 
connecting the two pulses that make up the profile. This is evidence that PSR B1855+09 is an aligned rotator, a question which has generated much discussion and disagreement (see, for example, Segelstein et al. (1986), Thorsett \& Stinebring (1990), and Rankin (1990)).

The linearly polarized emission presented here is in agreement with previously published profiles (see Yan et al. (2011) and Dai et al. (2015)), which shows relatively low levels of linear polarization with components that coincide with all of the components in the total intensity profile, however, we are able to resolve multiple additional components. The circularly polarized emission we present is also largely in agreement with published profiles, although we note that we detect slightly less circularly polarized intensity.

\subsubsection{2 $430 \mathrm{MHz}$}

Just as at $1.4 \mathrm{GHz}$, the total intensity profile for PSR B1855+09 at $430 \mathrm{MHz}$ (Thorsett \& Stinebring, 1990) also shows two main pulses. With the improved sensitivity of this data set, we are able to detect every component in the $1.4 \mathrm{GHz}$ total intensity profile at $430 \mathrm{MHz}$, with the exception of the bridge of emission. The polarized emission, on the other hand, looks very different, as there's much less of it in general. The linearly polarized emission does seem to share some of the same properties as the $1.4 \mathrm{GHz}$ emission, as there are some distinguishable components that have corresponding total intensity components, but the circularly polarized emission looks very different. The only detectable phenomenology is weak RCP 
emission over much of both pulses.

\subsubsection{PSR J1903+0327}

\subsubsection{1 $2.1 \mathrm{GHz}$}

PSR J1903+0327's 2.1 GHz total intensity profile shows three components: a bright main component flanked by two weaker components. The linearly polarized emission shows that the weak, leading component is almost fully polarized, while the main component is coincident with the strongest linearly polarized component. There is also a moderate, RCP component coincident with the main pulse peak, which is the only detectable circularly polarized emission.

\subsubsection{2 $1.4 \mathrm{GHz}$}

PSR J1903+0327's 1.4 GHz total intensity profile is very similar to the 2.1 $\mathrm{GHz}$ total intensity profile, showing the same three components. The linearly polarized emission is not strikingly different either, although at $1.4 \mathrm{GHz}$, the leading component shows a very low polarization fraction. Also, the brightest linearly polarized component, while still coincident with the total intensity peak, appears to be much wider that at $2.1 \mathrm{GHz}$. The circularly polarized emission appears to be nearly identical at $1.4 \mathrm{GHz}$, showing a RCP component coincident with the total intensity peak. 


\subsubsection{PSR J1910+1256}

\subsubsection{1 $2.1 \mathrm{GHz}$}

The $2.1 \mathrm{GHz}$ profile for PSR J1910+1256 consists of one pulse with one bright, primary component preceded by a low-intensity tail and followed by a small bump leading into another long tail. The longer, trailing tail is almost completely polarized, while the leading trail does not show evidence for polarized emission. The remainder of the linear intensity profile consists of two components that are roughly, though not exactly, coincident with the bright, primary total intensity component. The circularly polarized profile contains one bright LCP peak that is coincident with the brightest linear polarization peak, followed by a reversal in the sense of circularly polarized emission, leading to a RCP peak which trails off into a long tail.

\subsubsection{2 $1.4 \mathrm{GHz}$}

PSR J1910+1256's 1.4 GHz profile (Stairs et al., 2005; Lorimer et al., 2006; Gonzalez et al., 2011) looks very similar to its $2.1 \mathrm{GHz}$ profile in that there is still a one bright, primary component preceded by a low-intensity tail and followed by a small bump leading into another long tail, and indeed both the linear and circular polarization profiles share many of the same overall characteristics of the $2.1 \mathrm{GHz}$ polarized emission. For example, the linearly polarized emission still shows two main components roughly, yet not exactly coinciding with the brightest total intensity component and emission that extends into the trailing tail, and the circularly polarized emission still contains one bright LCP peak that is coincident with the 
brightest linear polarization peak, followed by a reversal in the sense of circularly polarized emission, leading to a RCP peak which trails of into a long tail, yet there are some small, yet nonetheless interesting differences. For example, the linear intensity profile now shows a very small component preceding the two main components, and the long linearly polarized tail of the $2.1 \mathrm{GHz}$ emission is seen at $1.4 \mathrm{GHz}$ to be two components, the first of which roughly aligns with the bump seen in the total intensity profile. Also of note is that the ratio of peak linearly polarized intensity to peak circularly polarized intensity has reversed: at $2.1 \mathrm{GHz}$, the circularly polarized peak was more intense than the linearly polarized peak, whereas the opposite is true at $1.4 \mathrm{GHz}$.

\subsubsection{PSR J1911+1347}

\subsubsection{1 $2.1 \mathrm{GHz}$}

The $2.1 \mathrm{GHz}$ total intensity profile of PSR J1911+1347 consists of a very bright pulse neighbored on either side by lower intensity components. Inspection of these lower-intensity components reveals that the final component of the total intensity profile appears to be connected to the rest of the profile. The linear polarization profile shows that this component is nearly $100 \%$ polarized, and that there is a strong linearly polarized component coincident with the total intensity peak. Also coincident with the total intensity peak is a strong LCP peak, followed by a sense reversal and a very weak RCP component. There also appears to be another, very weak RCP peak coincident with the final total intensity component. 


\subsubsection{2 $1.4 \mathrm{GHz}$}

PSR J1911+1347's 1.4 GHz total intensity profile (Lorimer et al., 2006) is strikingly similar to its $2.1 \mathrm{GHz}$ counterpart, and we show that bridge of emission seen connecting the final component to the rest of the total intensity profile seen at 2.1 GHz is even more evident at $1.4 \mathrm{GHz}$. The linear intensity profile at $1.4 \mathrm{GHz}$ is nearly identical to the $2.1 \mathrm{GHz}$ linear intensity profile, save the preceding components that lead the linearly polarized peak. Unsurprisingly, the circularly polarized emission has also remained largely unchanged, with the weak RCP components being even more evident.

\subsubsection{3 $430 \mathrm{MHz}$}

PSR J1911+1347's 430 MHz profile does not look wholly unlike its $1.4 \mathrm{GHz}$ profile as the primary component appears to be mostly unchanged, however what was seen as a long precursor tail appears as two separate components at $430 \mathrm{MHz}$. We do not detect linear polarization, but we do detect circular polarization, which shows a LCP peak coinciding with the main pulse and is followed by a possible sense reversal and RCP peak, although these features are extremely weak.

\subsubsection{PSR J1923+2515}

\subsubsection{1 $1.4 \mathrm{GHz}$}

The 1.4 GHz profile for PSR J1923+2515 that was reported upon its discovery (Lynch et al., 2013) showed a fairly simple, two-component pulse, however the 
detection of an additional component preceding the $2.1 \mathrm{GHz}$ total intensity peak by $\sim 0.35$ rotations coupled with the detection of that same additional component at $820 \mathrm{MHz}$ suggests that there may be more to PSR J1923+2515's $1.4 \mathrm{GHz}$ emission than was originally reported. The improved sensitivity afforded by AO reveals that and other lower-intensity components. In fact, there is evidence for another component preceding the aforementioned feature, as well as yet another component following the aforementioned feature (the latter of which is also seen in the $2.1 \mathrm{GHz}$ profile).

The linear polarization profile shows many components that correspond to components in the total intensity profile, including three major components that roughly align with the two major components of the total intensity profile. These components are connected with a bridge of linearly polarized emission. PSR J1923+2515 does not show very much circularly polarized emission, however, there are two RCP components that align with two corresponding linearly polarized components.

\subsubsection{2 $430 \mathrm{MHz}$}

The $430 \mathrm{MHz}$ profile for PSR J1923+2515 shows a similar morphology to the $1.4 \mathrm{GHz}$ profile in that there are two main components preceded by a weak component (the same component seen at 2.1 and $820 \mathrm{MHz}$ in Lynch et al. (2013)), meaning that this component is seen consistently from $430 \mathrm{MHz}$ to $2.1 \mathrm{GHz}$. At $2.1 \mathrm{GHz}$, the first of the two main components is much brighter than the trailing component, and as the frequency of observation decreases, so does the intensity 
of the first main component with respect to the second. We show that this trend continues at $430 \mathrm{MHz}$.

The linear polarization profile shows one component with a long, leading tail and a peak that corresponds to the trailing component of the total intensity profile. The circular polarization profile appears to mirror the linear polarization profile, although in place of a leading tail, there are three RCP components whose intensities increase with pulse phase.

\subsubsection{PSR B1937+21}

\subsubsection{1 $2.1 \mathrm{GHz}$}

The most sensitive 2.1 GHz polarization profile for PSR B1937+21 was presented in Dai et al. (2015), however, the profiles were significantly affected by dispersive smearing, an issue which is not shared by the profiles presented here. For example, we see the component following the total intensity peak that is commonly seen in coherently dedispersed PSR B1937+21 profiles at multiple frequencies. We also see the very complex microcomponents in the total intensity profiles, except we show another, very faint component after the main pulse that does not appear in previously published profiles. We also see that the microcomponent preceding the total intensity peak by $\sim 0.75-0.8$ is in fact two components, and are able to resolve the microcomponent centered at a pulse phase of $\sim 0.25$ enough to see that it is in fact three components.

The linearly polarized emission at $2.1 \mathrm{GHz}$ shows that the main pulse is com- 
prised of three components which, like the corresponding total intensity pulse, is preceded by a long tail. The lower-intensity pulse has two linearly polarized components, however the second of those components is extremely weak. The microcomponents show significant polarization fractions, especially the microcomponents spanning pulse phases of $\sim 0.2-0.4$. The microcomponents do not show any detectable circularly polarized emission, while the lower-intensity pulse has a coincident RCP peak, and the main pulse has a RCP peak, followed by a reversal in the sense of circular polarization coincident with the total intensity peak, then a LCP peak.

\subsubsection{2 $1.4 \mathrm{GHz}$}

The polarization profile of PSR B1937+21 has been studied extensively at 1.4 GHz (see, for example Dai et al. (2015); Ord et al. (2004); Yan et al. (2011), and more), and our results are broadly in good agreement with that consensus, although we are able to resolve the rich microcomponents with coherently dedispersed data for the first time. We see the same microcomponents detected at $1.4 \mathrm{GHz}$, although with much higher SNR. In fact, we see that the microcomponent occurring at a pulse phase of $\sim 0.4$ appears to be two components. The increased SNR at $1.4 \mathrm{GHz}$ compared to $2.1 \mathrm{GHz}$ allows us to more readily see the widths of these microcomponents, which reveals that PSR B1937+21 is "on" for at least a large majority of its rotation, a conclusion that would be contrary to that drawn from data that is not sensitive enough to resolve these microcomponents.

The polarization properties of our observations again reflect those previously 
published. The linearly polarized emission shows the same basic structure as at 2.1 $\mathrm{GHz}$, however we can much more clearly see just how linearly polarized many of the microcomponents are. In contrast, none of the microcomponents show any circular polarization.

\subsubsection{PSR J1944+0907}

\subsubsection{1 $2.1 \mathrm{GHz}$}

The 2.1 GHz PSR J1944+0907 data have comparatively low SNR, making us unable to report a detailed description of its $2.1 \mathrm{GHz}$ pulse profile. Still, we can see that the $2.1 \mathrm{GHz}$ pulse profile is relatively broad, and includes two components of roughly equal peak intensities. We cannot report any significant detection of linear or circular polarization.

\subsubsection{2 $1.4 \mathrm{GHz}$}

PSR J1944+0907's 1.4 GHz profile is incredibly complex, showing at least eight distinguishable components, including a low-intensity trailing component. The width of the $1.4 \mathrm{GHz}$ total intensity profile is similar to that of the $2.1 \mathrm{GHz}$ profile, however the leading pulse component is seen to be significantly brighter than any other component in the profile.

The linear polarization profile is similarly complex; perhaps more so. Indeed, there are eleven distinct components. Although many of the components are apparently coincident with corresponding components in the total intensity profile, the 
density of profile components makes it difficult to ascribe one component to another (or rather, makes it difficult not to), which would call into question the physical relevance of any such ascription.

Unsurprisingly, the circular polarization profile is also very complex, with at least three reversals in the sense of circularly polarized emission. The two brightest components are LCP peaks, although there are many other lower-intensity LCP and RCP components.

\subsubsection{3 $430 \mathrm{MHz}$}

Champion et al. (2005) shows that PSR J1944+0907's 430 MHz total intensity profile consists of two main components that, together, form a profile that looks very similar to the $1.4 \mathrm{GHz}$ profile, albeit with far less complexity. Our data agrees with this, however, we are able to barely detect a tail on the trailing edge of the profile.

As with the $1.4 \mathrm{GHz}$ data, PSR J1944+0907 shows linearly polarized emission throughout virtually the entire total intensity pulse at $430 \mathrm{MHz}$, although again there is far less structure apparent to the linear polarization profile. We see at least 3 components, one of which aligns with the brightest total intensity component.

The circularly polarization profile looks very different at $430 \mathrm{MHz}$ than it does at $1.4 \mathrm{GHz}$ in that the emission is almost entirely RCP with one change in the sense of circularly polarized emission. 


\subsubsection{PSR J1949+3106}

\subsubsection{1 $2.1 \mathrm{GHz}$}

Multi-frequency polarimetry for PSR J1949+3106 was taken upon its discovery (Deneva et al., 2012), and what was found was that PSR J1949+3106 does not show much polarization at all at both $820 \mathrm{MHz}$ and $1.4 \mathrm{GHz}$. This is also true for PSR J1949+3106 at $2.1 \mathrm{GHz}$, although there is some linearly polarized emission spanning the duration of the total intensity emission. There is no detectable circularly polarized emission.

Deneva et al. (2012) reported a total intensity profile with two bright peaks, and that the first component has a steeper spectral index than the last. They report that these characteristics are consistent over both observation frequencies $(820 \mathrm{MHz}$ and $1.4 \mathrm{GHz}$ ), and we find that these characteristics are consistent with the $2.1 \mathrm{GHz}$ emission.

\subsubsection{2 $1.4 \mathrm{GHz}$}

Our 1.4 GHz data are consistent with the profile reported in Deneva et al. (2012), although the improved sensitivity shows that the linear polarization profile consists of at least 3 components, the brightest on the leading edge and another bright component on the trailing edge. The circularly polarized emission is weak, but shows a RCP peak coinciding with the trailing total intensity peak followed by a reversal of the sense of circular polarization, then a LCP peak coinciding with the trailing linear polarization peak. 


\subsubsection{PSR B1953+29}

\subsubsection{1 $1.4 \mathrm{GHz}$}

The 1.4 GHz profile for PSR B1953+29 has been well studied (Boriakoff et al. (1983), Kramer et al. (1998), and Gonzalez et al. (2011)), and includes a bright, multi-component pulse preceded by a low-intensity component. The linear polarization profile shares much of the same shape as the total intensity profile as every total intensity component has a corresponding linearly polarized component. The same can be said of the circularly polarized emission, with the exception of the first two total intensity components. The circular polarization profile starts as LCP, then reverses sense and finished the profile as RCP. Interesting to note is the fact that even though the linearly and circularly polarized emission share very similar shapes, their components appear to be slightly offset from each other.

\subsubsection{PSR J1955+2527}

\subsubsection{1 $1.4 \mathrm{GHz}$}

As with PSR J1949+3106, polarimetry data for PSR J1955+2527 was taken upon its discovery (Deneva et al., 2012), although the data did not have a high enough SNR to detect any linear or circular polarization. Our data are sensitive enough to detect polarized emission, and it is indeed at a low enough level to be consistent with no detection at a lower SNR. Both the linear and circular polarization profiles show one broad pulse spanning nearly the entire duration of the total 
intensity pulse. As for the total intensity profile, it is relatively simple, though there does appear to be a bump on the leading edge. This is interesting because Deneva et al. (2012) proffered the possibility of an "unresolved bump on the leading edge of the main pulse and/or a slight bump at the very top of the pulse" as a possible explanation for non-gaussian characteristics of the pulse profile.

\subsubsection{PSR J2017+0603}

\subsubsection{1 $2.1 \mathrm{GHz}$}

The 2.1 GHz total intensity profile for PSR J2017+0603 is broadly comprised of two parts, both consisting of multiple components themselves. The first of these two parts is preceded by a tail of emission and is followed by a bridge of emission that connects the two major parts of the total intensity emission. The linear polarization profile is similar in many ways to the total intensity profile, as the components of the linear polarization profile appear to be able to be mapped to components of the total intensity profile bijectively. The relative intensities of these components, however, are not related to the relative intensities of their total intensity counterparts in general. The circular polarization profile is considerably simpler and weaker than the total intensity and linear polarization profiles, as there are only two resolvable RCP components, each coincident with brightest two components of the total intensity profile. 


\subsubsection{2 $1.4 \mathrm{GHz}$}

The $1.4 \mathrm{GHz}$ total intensity profile looks very similar to the $2.1 \mathrm{GHz}$ total intensity profile: we see two main parts, connected by a bridge of emission and preceded by a tail of emission. The exceptional SNR of PSR J2017+0603's 1.4 GHz profile reveals that as complex as the $2.1 \mathrm{GHz}$ emission is, the $1.4 \mathrm{GHz}$ emission shows even more complexity. The linearly polarized emission varies from almost perfectly tracing out the total intensity profile with $100 \%$ of the emission being linearly polarized, to straying significantly from the total intensity profile, showing almost no polarized emission. There appears to be no detectable circularly polarized emission, save for two very weak RCP pulses aligning with the most intense components of each of the main parts of the total intensity profile.

\subsubsection{3 $430 \mathrm{MHz}$}

As with the 2.1 and $1.4 \mathrm{GHz}$ emission, we again see two parts to the total intensity emission at $430 \mathrm{MHz}$, however (perhaps due to the low SNR), we see neither a leading tail nor a bridge of emission. Moreover, and again perhaps due in part to the low SNR, the total intensity profile shows significantly less structure at $430 \mathrm{MHz}$ than at higher frequencies. Further differences between the $430 \mathrm{MHz}$ profile and the higher frequency profiles, and ones that are not due to a low SNR, are seen in the linear polarization profile. Linearly polarized emission, which at times constituted nearly $100 \%$ of the total 2.1 and $1.4 \mathrm{GHz}$ emission, is not significantly detected in any part of the pulse profile. We do, however, detect a RCP component 
of emission that spans much of the latter part of the total intensity profile.

\subsubsection{PSR J2019+2425}

\subsubsection{1 $1.4 \mathrm{GHz}$}

The 1.4 GHz profile for PSR J2019+2425 consists of 3 parts: a main, dualcomponent part flanked by two lower-intensity components (Nice et al., 2001). The two flanking components, which are very similar in total intensity, yet are polar opposites in linear polarization. The component preceding the main pulse shows no detectable linear polarization, whereas the component following the main pulse appears to be $100 \%$ linearly polarized. This morphology persists at low frequencies (Nice et al., 1993). The main pulse itself shows two linear polarization components, one on the leading edge of the pulse, and one on the trailing edge of the pulse. Interestingly, the local minimum of the linear intensity profile between these two components is coincident with one of the peaks of total intensity emission. There is only one weak LCP component to the circularly polarized emission. This component is coincident with the aforementioned linear polarization local minimum.

\subsubsection{PSR J2033+1734}

\subsubsection{1 $1.4 \mathrm{GHz}$}

PSR J2033+1734 was discovered with the AO at $430 \mathrm{MHz}$, and its profile was reported to be a bright pulse followed by a long tail (Ray et al., 1996). This phe- 
nomenology also accurately describes PSR J2033+1734's 1.4 GHz profile, although the "tail" appears to be more accurately described as a flat, shelf-like feature, followed by a more conventional, gaussian component. The profile shows significant linear polarization, which mostly occurs on the leading edge of the pulse, leaving the trailing components unpolarized. There is a moderate LCP leak, followed by a sense reversal coinciding with the total intensity peak, leading to a more intense RCP peak. As with the linear polarization, there is no detectable circular polarization associated with the trailing components of the total intensity emission.

\subsubsection{2 $430 \mathrm{MHz}$}

As described above, PSR J2033+1734 was reported upon its discovered with the $\mathrm{AO}$ at $430 \mathrm{MHz}$ to have a profile that consists of a bright pulse followed by a long tail (Ray et al., 1996). We report this same general structure, but our coherently dedispersed data show the pulse is much narrower than initially reported, and that the long tail is inherent to the pulsar's emission, rather than being caused by external processes caused by things such as propagation through the interstellar medium. The linear polarization profile is simple, showing one component roughly aligning with, yet slightly preceding the total intensity main pulse. The circular polarization profile is similarly simple, as there is again only one detectable component: a RCP pulse roughly aligned with, yet slightly following the main pulse. 


\subsubsection{PSR J2043+1711}

\subsubsection{1 $1.4 \mathrm{GHz}$}

Upon its discovery, PSR J2043+1711's $1.4 \mathrm{GHz}$ profile was reported to be "complex, with several pulsed components" (Guillemot et al., 2012a). Improved sensitivity shows a profile that, to say the least, confirms that statement. What appeared to be the brightest single component is revealed to be two components, and the trailing component is shown not only to have a steep drop in the emission that was previously unresolved, but also to not, in fact, be the trailing component after all, as a very weak pulse is detected following it. The linear polarization profile shows similar complexity, with the brightest components being almost $100 \%$ polarized, while other components in the total intensity profile show no corresponding linear polarization at all. For as intense and complex as the linear polarization profile appears to be, the circular polarization profile is much more simple: one RCP component aligned with the brightest total intensity component and two weak LCP components aligning with other total intensity components.

\subsubsection{2 $430 \mathrm{MHz}$}

PSR J2043+1711's profile looks very different at $430 \mathrm{MHz}$ than it does at 1.4 GHz. Where at higher frequencies, there was a trailing component connected to the main pulse profile by a weak bridge of emission, both the bridge and the trailing component are seen at all at $430 \mathrm{MHz}$. Instead, we see 5 relatively sharp components. The linear polarization profile resembles the total intensity profile, with every total 
intensity component except the last one showing a corresponding linearly polarized component. The circular polarization profile almost perfectly mirrors the linearly polarized emission in RCP, although it is slightly less intense.

\subsubsection{PSR J2214+3000}

\subsubsection{1 $2.1 \mathrm{GHz}$}

PSR J2214+3000's 2.1 GHz profile consists primarily of two pulses, the peaks of which are separated by almost exactly half a rotation. The brightest of these is made up of one bright component preceded by a much dimmer component. The other pulse is comprised of three components. Following the brighter pulse, however, we are able to detect another, very weak feature. This feature is very narrow and approximately as bright as the component leading the brightest component in the total intensity profile. The linear polarization profile shows a similar structure to the total intensity profile. The leading component of the brightest pulse is nearly $100 \%$ polarized, whereas the following component is much less polarized. The dimmer of the two main pulses in the total intensity profile also shows significant linear polarization, although we are unable to detect linear polarization from its trailing component. There is no detectable circular polarization throughout the pulse profile.

\subsubsection{2 $1.4 \mathrm{GHz}$}

PSR J2214+3000's $1.4 \mathrm{GHz}$ profile looks very similar to the $2.1 \mathrm{GHz}$ profile (unsurprising, as this general shape appears to persist down to frequencies as low 
as $820 \mathrm{MHz}$ (Ransom et al., 2011)). Again, we see two bright pulses separated by about half a rotation, and again, we are able to detect a very faint pulse trailing the brightest of the total intensity pulses. At $1.4 \mathrm{GHz}$, however, we are also able to detect a tail leading this very faint pulse. The linear polarization profile still shows a similar structure to the total intensity profile, and the leading components of both pulses are very highly polarized, however at $1.4 \mathrm{GHz}$ we find that the brightest component in the total intensity profile is much more highly polarized. We also find that the trailing component in the dimmer total intensity pulse does have corresponding linear polarization. We are also able to see that the faint, solitary

pulse is very highly polarized. There is not much circular polarization to speak of, although there does seem to be a broad RCP pulse and LCP pulse coincident with the brighter and dimmer total intensity pulses, respectively.

\subsubsection{PSR J2229+2643}

\subsubsection{1 $1.4 \mathrm{GHz}$}

The first published profiles of PSR J2229+2643 at $1.4 \mathrm{GHz}$ (Camilo et al., 1996b) had significantly lower SNR than the profile published in this work, however, since PSR J2229+2643's 1.4 GHz profile is relatively smooth and broad, the increase in sensitivity serves more of a confirmation of previously published profiles than a revelation of previously hidden details. The linear polarization profile contains two components: one relatively sharp component aligned with the total intensity peak, and one smaller component on the leading edge of the profile. The circular 
polarization profile shows a RCP peak, followed by a sense reversal, a LCP peak, another sense reversal, and an final RCP peak. The LCP peak appears to be aligned with the total intensity peak.

\subsubsection{2 $430 \mathrm{MHz}$}

Since the 1.4 GHz profile was relatively smooth and broad, and therefore served as more as a confirmation of previously published profiles, it is perhaps not a surprise that the same is true at $430 \mathrm{MHz}$. Indeed, the total intensity profile shows the same general shape at $430 \mathrm{MHz}$ as it did at $1.4 \mathrm{GHz}$, a phenomenology that has already been established (Camilo et al., 1996b). For as little as the total intensity profile changes from $1.4 \mathrm{GHz}$ to $430 \mathrm{MHz}$, the polarization profiles at $430 \mathrm{MHz}$ are starkly different. We detect no linear polarization, and the only circular polarization we detect is a weak LCP peak that slightly trails the total intensity peak.

\subsubsection{PSR J2234+0611}

\subsubsection{1 $1.4 \mathrm{GHz}$}

PSR J2234+0611's $1.4 \mathrm{GHz}$ profile appears to be relatively narrow, however closer inspection reveals that it radiates over a substantial fraction of its rotation. The profile shows many components, however most of the components have peak fluxes that are an order of magnitude or two lower than the peak flux of the profile as a whole. These low-intensity components show a high degree of linear polarization in general, although the trailing component appears to be unpolarized. The circular 
polarization is weak, and shows two LCP peaks that are coincident with the two strongest peaks in the linear polarization profile.

\subsubsection{PSR J2234+0944}

\subsubsection{1 $2.1 \mathrm{GHz}$}

The total intensity profile for PSR J2234+0944 at 2.1 GHz appears to be made up primarily of to two separate pulses, however, the high SNR of the data show that the pulses that comprise the profile are in fact connected by a bridge of emission. Interestingly, a tail of emission leads into this bridge from both sides. The profile shows moderate linear polarization, with a dual-component pulse coincident with the larger of the total intensity pulses and a very low-intensity component coincident with the smaller of the total intensity pulses. The circularly polarized emission is simple, with two components aligning roughly with the total intensity pulses. These pulses are both LCP, and therefore PSR J2234+0944 does not display a detectable reversal in the sense of circularly polarized emission at $2.1 \mathrm{GHz}$.

\subsubsection{2 $1.4 \mathrm{GHz}$}

The total intensity profile for PSR J2234+0944 at $1.4 \mathrm{GHz}$ is very similar to its $2.1 \mathrm{GHz}$ counterpart in that it also appears to be made up primarily of to two separate pulses that are connected by a bridge of emission, however, we are able to detect microcomponents preceding the total intensity peak. The first of these microcomponents does not appear to be polarized, while the second appears to be 
100\% linearly polarized. The polarized emission coincident with the high-intensity pulses in the total intensity profile are also largely similar to the corresponding 2.1 $\mathrm{GHz}$ components, however we note that a tail emission precedes the final linearly polarized emission, and the higher SNR of the $1.4 \mathrm{GHz}$ profile reveals a reversal in the sense of circularly polarized emission.

\subsubsection{3 $430 \mathrm{MHz}$}

Although the $430 \mathrm{MHz}$ profile for PSR J2234+0944 resembles the high-frequency profiles in that it is broadly made up of two pulses, we show that the trailing pulse is stronger at $430 \mathrm{MHz}$ whereas the preceding pulse was stronger at higher frequencies, indicating that the spectrum of the trailing pulse is steeper than the leading pulse. We detect a linearly polarized component coincident with the first total intensity peak as well as very weak RCP and LCP pulses corresponding to the two respective total intensity pulses, with a sense reversal in between.

\subsubsection{PSR J2317+1439}

\subsubsection{1 $1.4 \mathrm{GHz}$}

PSR J2317+1439's $1.4 \mathrm{GHz}$ profile shows a bright, extremely complex main pulse and a low-intensity postcursor. Kramer et al. (1998) reported that PSR J2317+1439's total intensity pulse consisted of 4 components (not counting the postcursor), but we show that is considerably more complex. They also report the presence of a precursor preceding the main pulse by about 0.4 rotations. This pre- 
cursor was reported to be about half as bright as the postcursor. As the postcursor is easily detectable in our data, we would expect such a precursor to also be easily detectable. As we do not detect it, we conclude that the precursor reported in Kramer et al. (1998) cannot be attributed to the pulsar's intrinsic emission.

The linear polarization profile also shows significant complexity, with the leading components being much more polarized than the trailing components, with the exception of the postcursor (which is nearly totally polarized). The circular polarization profile shows many components, but interestingly, they are all LCP, meaning that despite the complexity, there is no detectable change in the sense of circular polarization.

\subsubsection{2 $430 \mathrm{MHz}$}

Our total intensity profile for PSR J2317+1439 agrees well with previously reported $430 \mathrm{MHz}$ profiles (Camilo et al., 1993). The linear polarization profile is significantly simpler at $430 \mathrm{MHz}$ than at $1.4 \mathrm{GHz}$, as there are only two components: one broad component aligned with the first bright total intensity component, followed by a much weaker, much narrower component. The circular polarization profile, on the other hand, is much more complex at $430 \mathrm{MHz}$. While there was not one detectable reversal of sense of circular polarization at $1.4 \mathrm{GHz}$, there are many at $430 \mathrm{MHz}$. 


\subsubsection{Resulting Polarimetric Responses}

Recalling that the METM method was performed on profiles that had already been calibrated with the nominal PR created through the MEM procedure, the PRs shown in Figures 3.1 and 3.2 can be viewed as "residual PRs" (that is, corrections to the nominal, MEM-generated PR). Since new PRs were produced spanning a range of epochs, we can use them to describe the stability of the PR of AO's 1.4 and 2.1 $\mathrm{GHz}$ receivers.

First, though, it is important to be clear about the limitations of the analysis presented in this chapter. The ideal standard profiles to use would be ones that were produced using the MEM procedure. As such profiles were not available to us in this data set, we chose profiles that were consistent with previously published profiles. Inevitably, the standard profiles we chose will not be in perfect agreement with the true profiles of the sources we used to make the PRs. Any difference between the two will cause corresponding deviations that will propagate through the PRs made with the imperfect standard profiles, and since these imperfections will be caused by errors in the $\mathrm{PRs}^{2}$, we expect the errors introduced by using the METM procedure to be frequency-dependent.

Crucially, though, it is important to recall that using only the PR produced by the MEM procedure yielded profiles for PSRs J1713+0747 and B1937+21 that were not consistent epoch to epoch, whereas using the PRs created by the METM procedure produce profiles that are consistent with a profile that varies slightly from

\footnotetext{
${ }^{2}$ We assert this due to the stability that PSRs J1713+0747 and B1937+21's polarization profiles display in a number of published analyses over a wide range of epochs.
} 
the ideal standard profile. As the latter is undoubtedly preferable to the former, and as the (albeit slightly incorrect) standard profile does indeed appear to agree with published profiles, we can assume that errors caused by these considerations are small, especially compared to the errors that would have arisen if the METM procedure had not been implemented.

With these considerations in mind, we turn to the residual PRs themselves. In light of the previous discussion, and becuase the METM procedure treats the entire receiver chain as a black box, we do not aim to determine what specific components of the telescope receiver could provide an explanation for any behavior shown in the residual PRs. We also note that any errors in the residual PRs brought about by possible standard profile errors will be visited upon the PRs equally. Therefore, while those errors will affect the residual PRs in an absolute sense, the time-variability of the PRs will be largely unaffected.

We quantify the variability of the parameters that make up the residual PRs in two ways. First, for each parameter, we calculate the reduced $\chi^{2}$ for that parameter across all frequency channels on a given epoch. Assuming the standard profile errors are small, this describes how much the given parameter from the residual PR on that epoch varies from the nominal parameter from the residual PR produced by the MEM procedure. Second, for each parameter, we calculate the reduced $\chi^{2}$ for that parameter across all epochs in a given frequency channel. This describes how much the given parameter varies in that frequency channel on an epoch-by-epoch basis. Again, this variability will be largely unaffected by standard profile errors.

We find that the resulting reduced $\chi^{2}$ values, both while considering the varia- 
tion of parameters on a specific epoch, as well as in a specific frequency channel, are extremely large. While high reduced $\chi^{2}$ can be interpreted as an indication that the errors on parameters are underestimated, it also can serve as quantitative evidence for what can readily be seen by eye: the residual PR (and thereforethe PR) of AO is highly variable. We find this description of AO's PR to be consistent with both the $1.4 \mathrm{GHz}$ PRs and the $2.1 \mathrm{GHz}$ PRs.

\subsection{Discussion}

The profiles presented are the most sensitive pulsar polarization profiles to date, and this sensitivity reveals that the profiles of many millisecond pulsars have much more structure than may appear without careful inspection. That profile components can be hidden by insufficient SNR is not a new revelation, however, the detection of microcomponents in pulse profiles, particularly the microcomponents detected in PSRs B1937+21, J1713+0747, and J2234+0944 detected for the first time, challenges the very notion of "sufficient" SNR.

These microcomponents also complicate processes that aim to rigorously define "on-pulse" and "off-pulse" regions, as many such routines implicitly or explicitly assume that profile bins that have intensities which are "small" compared to the brightest components in the profile must be noise, an assumption which is refuted by the existence of microcomponents. This has implications for any process which requires the specification of "on-pulse" and "off-pulse" regions. Observers who would like to flux calibrate their observations by calculating radiometer noise, for example, 
may derive a substantially overestimated value for the radiometer noise present in an observation if an "off-pulse region" is not selected with great care with regard to the possible presence of microcomponents. It should be noted that since NANOGrav does not use the radiometer equation to calibrate their fluxes (Demorest et al., 2013), the reported fluxes of the NANOGrav pulsars are not affected by this effect.

This also reduces the utility of using the pulse width taken at some fraction of the total intensity peak as a proxy for pulsar beam width, especially when it is used in the context of a discussion about a pulsar's geometry, as the existence of microcomponents would have no effect on such a metric, and yet can have significant implications regarding a pulsar's geometry (as seen with PSR B1855+09).

We also present RM measurements for all sources at both 1.4 and $2.1 \mathrm{GHz}$ (where applicable) derived independently for each epoch. We note that for the sources where a RM could be determined independently from the $1.4 \mathrm{GHz}$ observations, the RM values are largely in agreement with each other. The source with the largest RM discrepancy versus receiver is PSR J1903+0327, whose RMs at 2.1 and at $1.4 \mathrm{GHz}$ differ by $1.5 \sigma$. While this discrepancy may be caused by statistical fluctuation, it is interesting to note that PSR J1903+0327 has the highest DM of any pulsar in the data set, and by far the highest DM of any pulsar for which we are able to measure RMs for 1.4 and $2.1 \mathrm{GHz}$ independently, which means that there is more interstellar material between Earth and the pulsar. As interstellar scattering is a frequency-dependent phenomenon, the radio waves scattered by the ISM will sample a different region of the ISM at different observing frequencies (Cordes et al., 2016). It is possible that the region sampled at $1.4 \mathrm{GHz}$ includes interstellar compo- 
nents and magnetic fields not sampled at $2.1 \mathrm{GHz}$, thereby changing the properties that govern Faraday rotation, and thus, the observed RM. We stress, though, that we put forth this explanation as another possibility rather than an assertion that it is indeed the cause of the variation in PSR J1903+0327's RM with respect to observing frequency. More observations would be necessary to determine the cause of any such variation.

Using equation ??, we can calculate the component of the Galactic magnetic field parallel to the line of sight to the source and use it to probe the structure of the Galaxy's magnetic field. We see that the magnetic field strengths are antisymmetric about the Galactic plane, with nearly all the magnetic field strengths above the plane being positive (towards Earth), and nearly all the magnetic field strengths below the plane being negative (away from Earth, see Figure 3.3). This structure suggests that the magnetic field in the Galactic halo is broadly dipolar, a phenomenology consistent with previous studies (Han et al., 1997; Xu \& Han, 2014). 

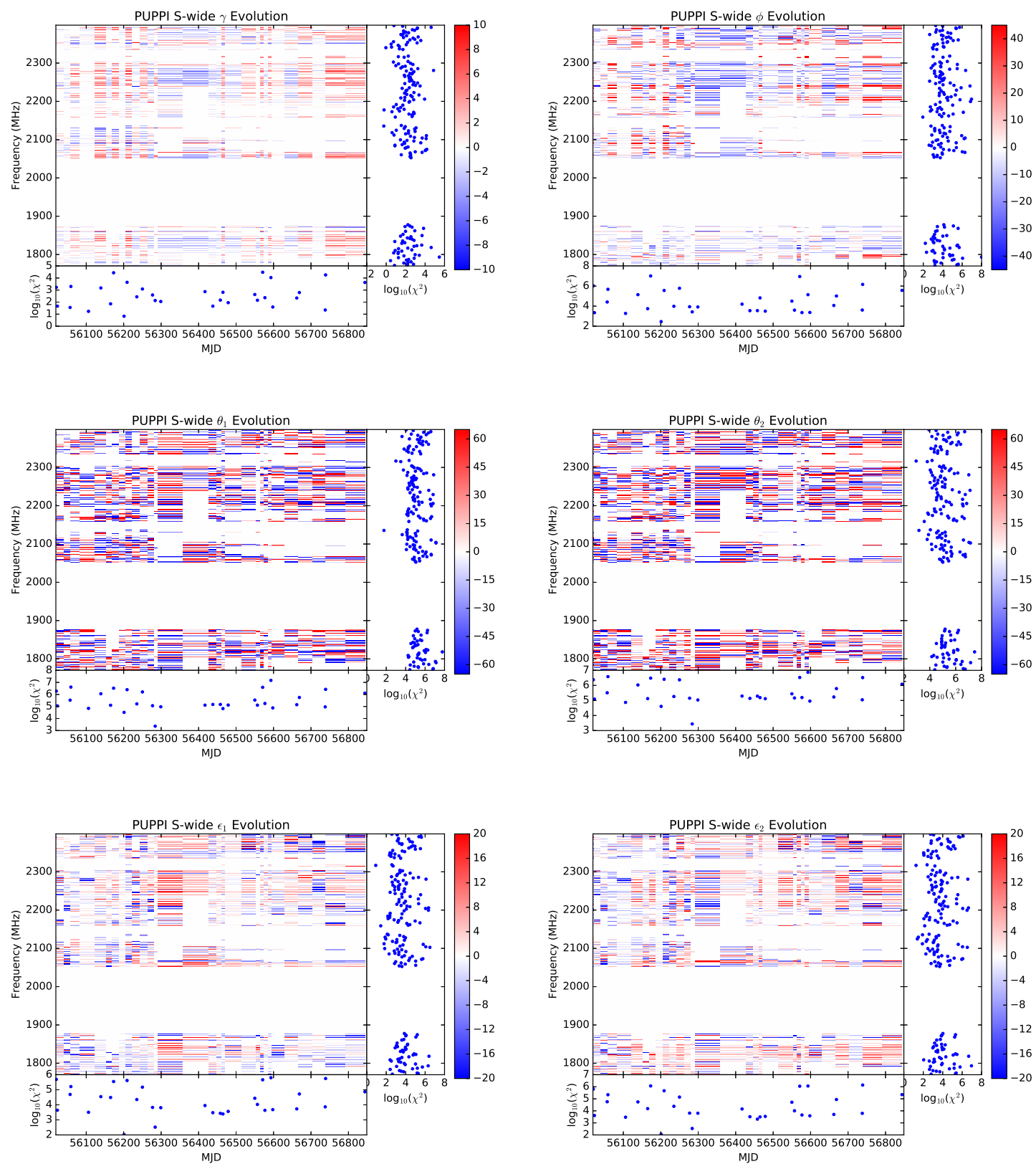

Figure 3.1: PRs of the S-wide receiver for both standard sources. For each color plot, the $\mathrm{x}$-axis is MJD and the $\mathrm{y}$-axis is observing frequency. To further highlight the differences, the color scales have been restricted. The lowest subplot of each plot shows the logarithm of the reduced $\chi^{2}$ of the given receiver parameter holding MJD constant, while the right-post subplot of each plot shows the logarithm of the reduced $\chi^{2}$ of the given receiver parameter in a specific frequency channel. Recall that the PRs are derived from observations that have already been calibrated with the MEM solution, and therefore the above plots do not show the absolute numerical values for each parameter versus frequency and MJD, but rather the difference between each parameter and its nominal MEM value over frequency and MJD. All angles are expressed in degrees. 

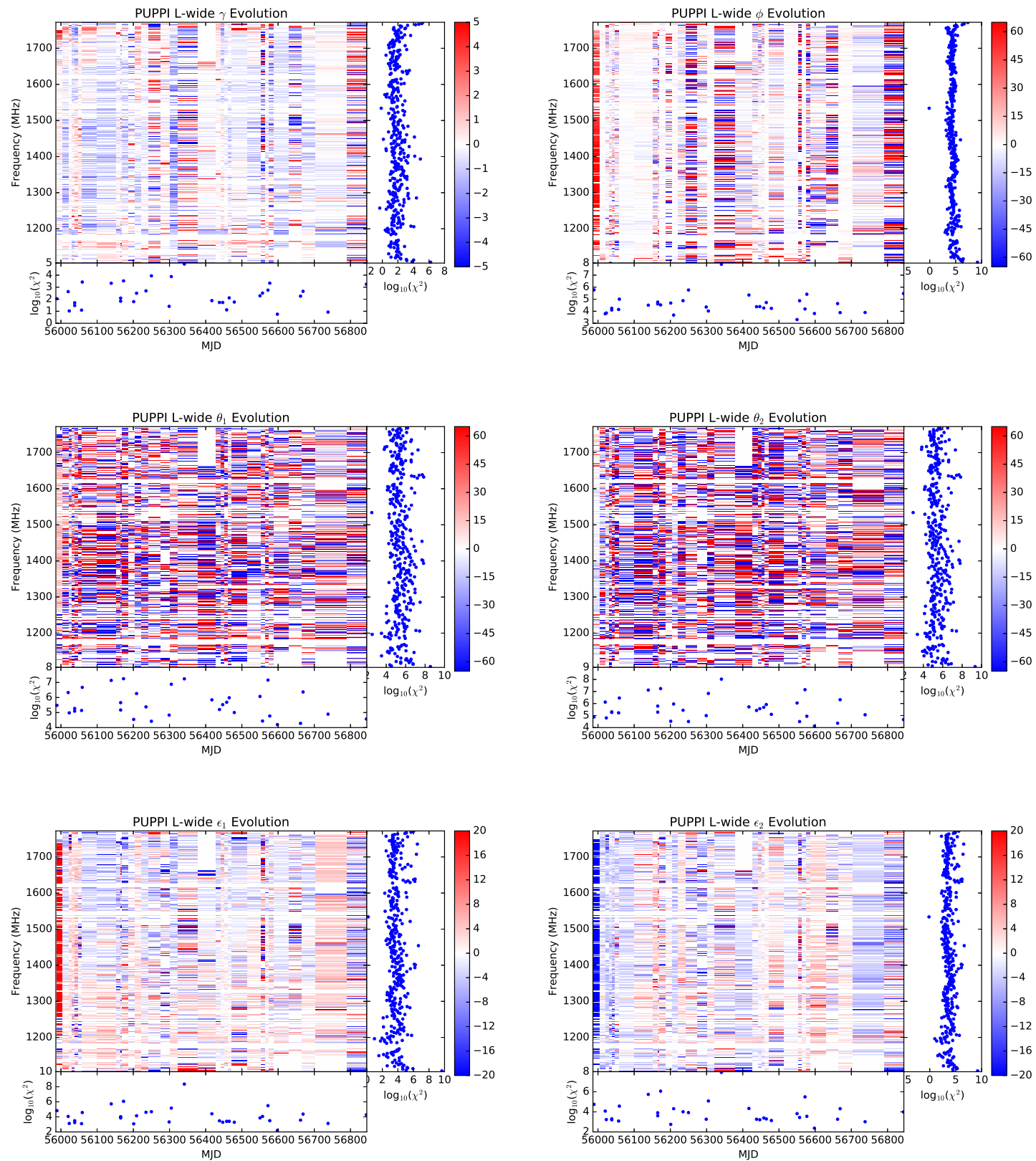

Figure 3.2: PRs of the L-wide receiver for both standard sources. For each color plot, the $\mathrm{x}$-axis is MJD and the $\mathrm{y}$-axis is observing frequency. To further highlight the differences, the color scales have been restricted. The lowest subplot of each plot shows the logarithm of the reduced $\chi^{2}$ of the given receiver parameter holding MJD constant, while the right-post subplot of each plot shows the logarithm of the reduced $\chi^{2}$ of the given receiver parameter in a specific frequency channel. Recall that the PRs are derived from observations that have already been calibrated with the MEM solution, and therefore the above plots do not show the absolute numerical values for each parameter versus frequency and MJD, but rather the difference between each parameter and its nominal MEM value over frequency and MJD. All angles are expressed in degrees. 


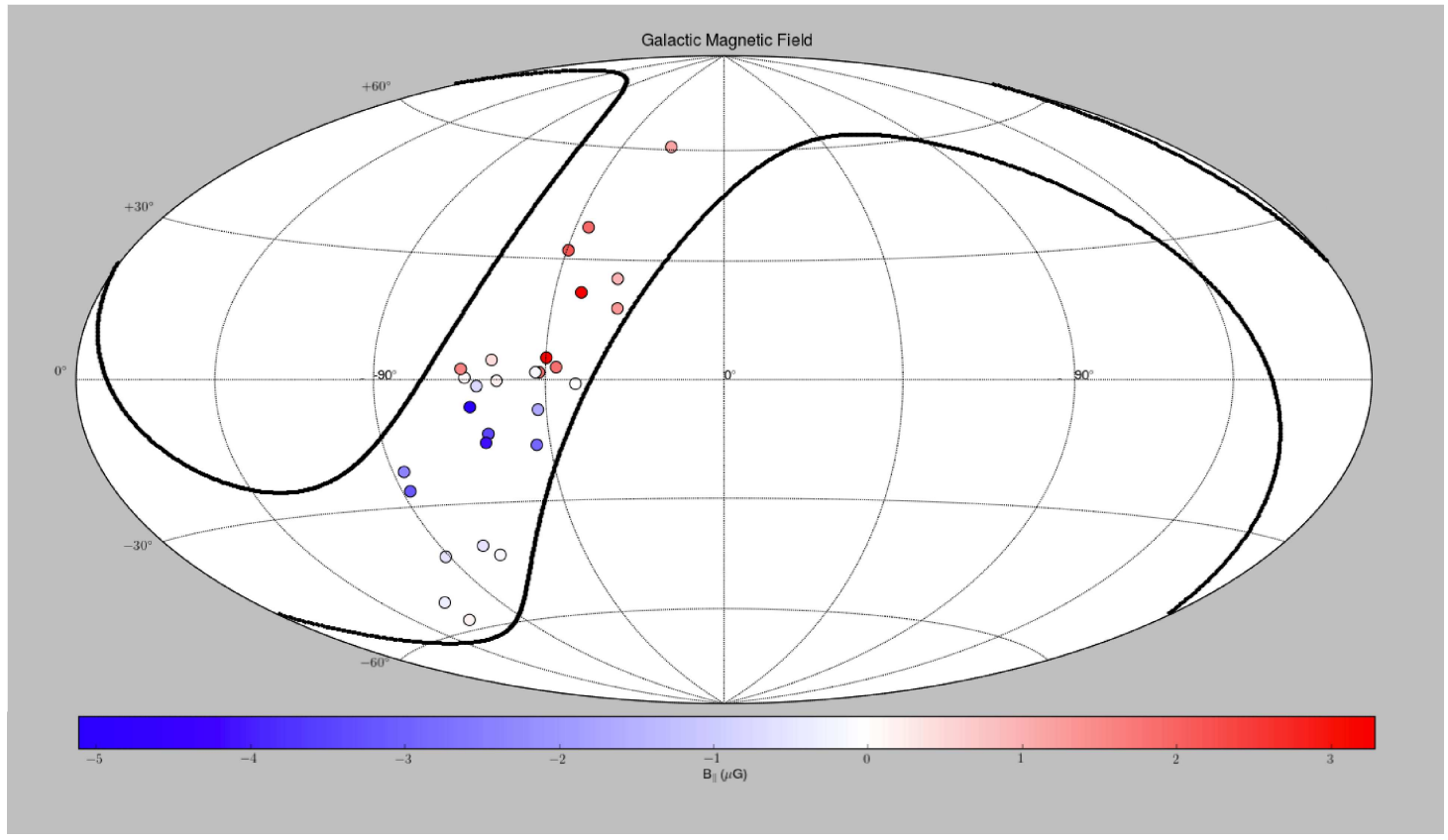

Figure 3.3: RM-derived values for the Galactic magnetic field parallel to the line of sight for each source as it appears on the sky in Galactic coordinates. Black curved lines show AO's visibility. Positive values denote a magnetic field pointing towards the Earth whereas negative values denote a magnetic field pointing away from the Earth. The asymmetry about a Galactic latitude of $0^{\circ}$ agrees with Galactic magnetic field models. 


\section{Chapter 4}

\section{X-Ray Observations of Black Widow Pulsars}

Published in ApJ as Gentile et al. 2014, ApJ, 783, 69 with co-authors Roberts, M. S. E., McLaughlin, M. A., Camilo, F., Hessels, J. W. T., Kerr, M., Ransom, S. M., Ray, P. S., and Stairs, I. H.

\subsection{Introduction}

Of the roughly 2000 radio pulsars known, about $10 \%$ are millisecond pulsars (MSPs) (Manchester et al., 2005) ${ }^{1}$, old neutron stars which have been spun-up, or 'recycled', through accretion of material from a companion (Alpar et al., 1982). Many details of this recycling process remain unknown, but it is clear that most known MSPs have degenerate white dwarf companions with masses between 0.2 and $1 M_{\odot}$. However, $\sim 1 / 6$ of the known MSPs in the Galactic field are isolated ${ }^{2}$. The process through which these MSPs were formed is unclear. One potentially important method is the ablation of the pulsar companion after the end of the recycling process by energetic particles and/or $\gamma$-rays produced in the pulsar magnetosphere (Ruderman et al., 1989).

The identification of MSPs as strong $\gamma$-ray sources (Abdo et al., 2010; Kuiper et al., 2000) motivates searches for radio pulsations in unidentified Fermi sources

\footnotetext{
${ }^{1}$ http://www.atnf.csiro.au/research/pulsar/psrcat

${ }^{2}$ See http://astro.phys.wvu.edu/GalacticMSPs/
} 
with spectral and temporal properties matching those of known $\gamma$-ray MSPs. Bangale et al. (2013) observed 49 sources at $350 \mathrm{MHz}$ with the Green Bank Telescope (GBT) and detected 17 MSPs, 10 of which were new discoveries and 16 of which are in binary systems, with seven of them having short orbital periods $\left(\mathrm{P}_{b}<1\right.$ day). Three of these pulsars (PSRs J0023+0923, J1124-3653, J1810+1744) and one (PSR J2256-1024) found in a 350-MHz GBT drift-scan survey (Boyles et al. (2013); Stairs et al., in prep) and re-detected in the Bangale et al. survey have very small companion masses $\left(\mathrm{M}_{\min }^{c} \ll 0.1 M_{\odot}\right)$ and three have pronounced radio eclipses, classifying them as black-widow pulsars (Stappers et al., 2003). One other pulsar discovered in this survey (PSR J2215+5135) has a short orbital period and eclipses, but a larger companion mass $\left(\mathrm{M}_{\min }^{c}=0.208 M_{\odot}\right)$ (Table 1). Optical observations of the companion suggest it is non-degenerate and nearly Roche-lobe filling and hence may be in an only temporary non-accreting, radio-emitting phase (Breton et al., 2013).

The first pulsar showing evidence for the ablation process was the original black-widow pulsar PSR B1957+20, which shows radio eclipses due to absorption in the wind of the companion and dramatic pulse delays around the time of eclipse due to propagation through the wind (Fruchter et al., 1990). XMM-Newton (Huang \& Becker, 2007) and Chandra observations (Stappers et al., 2003; Huang et al., 2012) revealed unresolved synchrotron emission that is variable throughout the orbit. On average, the orbital modulation is broadly sinusoidal, peaking near superior conjunction when the companion is between the pulsar and observer, but with a narrow dip over $\sim 0.1$ of the orbit at superior conjunction. This emission is interpreted as 
coming from an intrabinary shock of the pulsar's wind close to the nearly Roche-lobe filling companion's surface (van Kerkwijk et al., 2011). In addition, the Chandra observations resolved an extended tail of X-ray emission arising from the pulsar outflow shocking the interstellar medium, the first demonstration that millisecond pulsars can produce pulsar wind nebulae. Furthermore, magnetospheric pulsations in $\gamma$-rays and X-rays have been detected from the point source (Guillemot et al., 2012b).

An important link in the MSP formation scenario was made with the discovery of a radio pulsar (PSR J1023+0038) that showed evidence for having an accretion disk in the recent past (Archibald et al., 2009). This very fast $\left(P_{\text {spin }}=1.69 \mathrm{~ms}\right)$ eclipsing radio pulsar is in a 4.8-hr orbit around a nearly Roche-lobe filling, nondegenerate companion, and is the prototype of the 'redback' class of binary MSPs (Roberts, 2011). XMM-Newton (Archibald et al., 2010) and Chandra (Bogdanov et al., 2011) observations of this system revealed significant orbital variability over multiple consecutive orbits, with a pronounced dip in the X-ray flux at superior conjunction (orbital phases of $\sim 0.1$ to 0.4 ), when the pulsar is behind the companion and the intrabinary shock produced through the interaction of stellar outflows is obscured (Bogdanov et al., 2011). Because the angular extent of the pulsar as seen from the companion star is small, the width of this dip suggests that the X-ray emission region is much closer to the companion star than to the MSP. This evidence is strengthened further when considering the inclination of the binary system $\left(i \sim 46^{\circ}\right.$, constrained through optical radial velocity measurements (Archibald et al., 2009)). The X-ray spectrum consists of a dominant non-thermal component from the shock 
and at least one thermal component, likely originating from the heated pulsar polar caps. X-ray pulsations were also observed in the XMM-Newton data, indicating that some of the non-thermal point source emission is magnetospheric. For this source, no evidence for extended X-ray emission has been seen in the Chandra data (Bogdanov et al., 2011).

In general, the shock X-ray luminosity for a binary pulsar system will depend on the fraction of the wind intercepted by the companion, the spin-down energy loss rate $(\dot{E})$ of the pulsar, and both the post-shock magnetic field strength and the ratio of electromagnetic flux to kinetic energy flux, $\sigma$ (Arons \& Tavani, 1993; Kennel \& Coroniti, 1984). For PSR B1957+20, measurements of the X-ray orbital variability show that the efficiency of X-ray production at the shock is similar to that of pulsar wind nebulae around young pulsars, but it is not clear if this is true in all cases.

The body of knowledge regarding black-widow pulsars is still lacking. For example, intrabinary shocks can produce significant mass loss from black-widow companions by accelerating shocked particles out of the companion's Roche-lobe (Bogdanov et al., 2005), yet it remains to be shown whether this mass loss can be produced only from companions which are nearly filling their Roche-lobe. It also is not clear whether or not the winds from these pulsars are dominated by kinetic or magnetic energy.

Until very recently, studies were limited by the rarity of these systems. In the last few years however, many nearby systems have been discovered, more than tripling the known population (Ray et al., 2012). In Section 4.2, we summarize the observations and analysis procedures. In Section 4.3, we present the results of the 
spectral and light curve analyses. In Section 4.4, we offer conclusions. For each of these sources, we compare the X-ray properties to those of PSR B1957+20 and PSR J1023+0038, currently the two best-studied systems.

\subsection{Observations and Analysis}

We observed PSRs J0023+0923 (OBSID 14363), J1124-3653 (OBSID 13722), J1810+1744 (OBSID 12465), J2215+5135 (OBSID 12466), and J2256-1024 (OBSID 12467) for $15 \mathrm{ks}, 22 \mathrm{ks}, 22 \mathrm{ks}, 19 \mathrm{ks}$, and $22 \mathrm{ks}$ respectively, i.e., at least a full orbit in each case. The data were taken using Chandra's ACIS-S detector and analyzed using Chandra's data analysis suite, CIAO (version 4.2). Source regions were selected by first determining the locations of each source using radio timing positions. Background regions were chosen so that the regions were located on the same chip as the source and did not enclose any point sources.

Once source and background regions were selected, point-spread functions (PSFs) were then created for each source using CIAO's mkpsf function. Since the CIAO PSF library has PSFs for five discrete energies, we evaluated the PSF at energies which approximately correspond to where the count rate is a maximum. The energies chosen for PSRs J0023+0923, J1124-3653, J1810+1744, J2215+5135, and J2256-1024 were $0.75,1.25,1.5,0.8$, and $1 \mathrm{keV}$, respectively. Once energies were chosen, the mkpsf tool linearly interpolates a PSF from the five PSFs in the library. We then used these to compare the intensity of the source and PSF in two orthogonal directions. 
Table 4.1: Timing and X-ray Properties

\begin{tabular}{|c|c|c|c|c|c|c|c|c|c|c|}
\hline $\begin{array}{l}\text { Name } \\
\text { PSR }\end{array}$ & $\begin{array}{l}P_{\text {spin }} \\
(\mathrm{ms})\end{array}$ & $\begin{array}{c}\log _{10} \dot{E} \\
\left(\operatorname{erg~s}^{-1}\right)\end{array}$ & 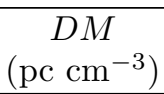 & $\begin{array}{c}\mathrm{nH} \\
10^{20} \mathrm{~cm}^{-2}\end{array}$ & $\begin{array}{c}D \\
(\mathrm{kpc})\end{array}$ & $\begin{array}{l}P_{\text {orb }} \\
\text { (hr) }\end{array}$ & $\begin{array}{l}M_{\min }^{c} \\
\left(\mathrm{M}_{\odot}\right)\end{array}$ & $\begin{array}{l}T_{\text {obs }} \\
(\mathrm{ks})\end{array}$ & $M J D_{o b s}$ & Cts \\
\hline J0023+0923 & 3.05 & 34.2 & 14.3 & 4.4 & 0.7 & 3.3 & 0.016 & 15 & 55893 & 43 \\
\hline J1124-3653 & 2.41 & 33.6 & 44.9 & 15.7 & 1.7 & 5.5 & 0.027 & 22 & 56118 & 138 \\
\hline $\mathrm{J} 1810+1744$ & 1.66 & 34.6 & 39.7 & 12.2 & 1.9 & 3.6 & 0.035 & 22 & 55740 & 55 \\
\hline $\mathrm{J} 2215+5135$ & 2.61 & 34.7 & 69.2 & 21.4 & 3.0 & 4.2 & 0.22 & 19 & 55697 & 133 \\
\hline J2256-1024 & 2.29 & 34.6 & 13.8 & 4.3 & 0.6 & 5.1 & 0.030 & 22 & 55788 & 141 \\
\hline$J 1023+0038$ & 1.68 & 34.6 & 14.3 & 18.0 & 1.3 & 4.8 & 0.2 & 83 & 55281 & 3270 \\
\hline$B 1957+20$ & 1.60 & 35.2 & 29.1 & $<1.0$ & 2.5 & 9.1 & 0.020 & 43 & 52081 & 370 \\
\hline
\end{tabular}

Note. - Timing and X-ray properties of the five Fermi-associated radio MSPs, including the pulsar spin period $\left(\mathrm{P}_{\text {spin }}\right)$, the logarithm of the spin-down energy loss rate $\left(\log _{10} \dot{\mathrm{E}}\right)$, dispersion measure (DM), neutral Hydrogen column density along the line of sight to the source (nH), distance to the pulsar (D), orbital period of the binary system $\left(\mathrm{P}_{\text {orb }}\right)$, minimum companion mass $\left(\mathrm{M}_{\min }^{c}\right)$, total observation duration $\left(\mathrm{T}_{o b s}\right)$, MJD of observation $\left(\mathrm{MJD}_{o b s}\right)$, and total background-subtracted counts (Cts). Due to the low number of background-subtracted counts, $\mathrm{nH}$ is estimated from DM (see text) and held fixed for each source. PSRs J1023+0038 and B1957+20 are shown for comparison. Timing properties are from 350-MHz observations with the GBT (see Bangale et al. 2013 and Hessels et al., in prep). Distances are estimated from the DM using the Cordes \& Lazio (2002) model for the Galactic electron density, except for PSR J1023+0038 which is from parallax measurements (Deller et al., 2012). 
Table 4.2: Spectral Fit Parameters

\begin{tabular}{|c|c|c|c|c|c|c|c|c|}
\hline $\begin{array}{l}\text { Name } \\
\text { PSR }\end{array}$ & $\begin{array}{c}k T \\
(\mathrm{eV})\end{array}$ & $\Gamma$ & $\begin{array}{c}F_{x} \\
\left(10^{-14} \mathrm{erg} \mathrm{s}^{-1} \mathrm{~cm}^{-2}\right)\end{array}$ & $\begin{array}{l}\log _{10} L_{x} \\
\left(\operatorname{erg~s}^{-1}\right)\end{array}$ & $\begin{array}{c}\epsilon \\
\left(10^{-5}\right)\end{array}$ & $\begin{array}{c}\text { Blackbody Flux } \\
\left(10^{-14} \mathrm{erg} \mathrm{s}^{-1} \mathrm{~cm}^{-2}\right)\end{array}$ & $\begin{array}{c}\text { Power-Law Flux } \\
\left(10^{-14} \mathrm{erg} \mathrm{s}^{-1} \mathrm{~cm}^{-2}\right)\end{array}$ & $\chi^{2} / \mathrm{DOF}$ \\
\hline \multicolumn{9}{|c|}{ Power-law Fit } \\
\hline J0023+0923 & $\ldots$ & $3.2_{-0.5}^{+0.6}$ & $3.0_{-0.9}^{+1.3}$ & 30.2 & 10 & $\ldots$ & $\ldots$ & $1.0 / 7$ \\
\hline $\mathrm{J} 1810+1744$ & $\ldots$ & $2.1_{-0.4}^{+0.4}$ & $2.5_{-0.7}^{+0.8}$ & 31.0 & 30 & $\ldots$ & $\ldots$ & $2.1 / 7$ \\
\hline $\mathrm{J} 2215+5135$ & $\ldots$ & $1.4_{-0.2}^{+0.2}$ & $9.7_{-2.0}^{+3.0}$ & 32.0 & 210 & $\ldots$ & $\ldots$ & $1.8 / 7$ \\
\hline $\mathrm{J} 2256-1024$ & $\ldots$ & $2.7_{-0.2}^{+0.2}$ & $5.3_{-0.6}^{+0.6}$ & 30.4 & 6 & $\ldots$ & $\ldots$ & $5.1 / 7$ \\
\hline J0023+0923 & $180_{-50}^{+60}$ & $\ldots$ & $1.4_{-0.9}^{+1.3}$ & 30.0 & 5 & $\ldots$ & $\ldots$ & $3.6 / 7$ \\
\hline J1124-3653 & $440_{-80}^{+100}$ & $\ldots$ & $3.1_{-2.3}^{+5.3}$ & 31.0 & 270 & $\ldots$ & $\ldots$ & $27.5 / 7$ \\
\hline $\mathrm{J} 1810+1744$ & $430_{-120}^{+130}$ & $\ldots$ & $1.2_{-0.9}^{+2.6}$ & 30.7 & 10 & $\ldots$ & $\ldots$ & $4.9 / 7$ \\
\hline $\mathrm{J} 2215+5135$ & $700_{-130}^{+150}$ & $\ldots$ & $5.3_{-3.7}^{+7.9}$ & 31.8 & 110 & $\ldots$ & $\ldots$ & $13.4 / 7$ \\
\hline $\mathrm{J} 2256-1024$ & $200_{-20}^{+20}$ & $\ldots$ & $3.2_{-1.6}^{+2.6}$ & 30.1 & 4 & $\ldots$ & $\ldots$ & $6.4 / 7$ \\
\hline \multicolumn{9}{|c|}{ Combined Fit } \\
\hline J0023+0923 & 150 & 1.5 & $1.9_{-0.6}^{+0.8}$ & 30.0 & 7 & $1.5_{-0.5}^{+0.4}$ & $1.0_{-0.6}^{+0.7}$ & $2.7 / 7$ \\
\hline $\mathrm{J} 1124-3653$ & 150 & $1.3_{-0.4}^{+0.5}$ & $5.4_{-2.5}^{+4.0}$ & 31.3 & 470 & $2.3_{-1.0}^{+1.0}$ & $5.4_{-2.7}^{+4.4}$ & $9.3 / 6$ \\
\hline $\mathrm{J} 2215+5135$ & 150 & $1.2_{-0.3}^{+0.4}$ & $8.1_{-3.3}^{+5.4}$ & 31.9 & 170 & $1.2_{-0.7}^{+1.0}$ & $9.2_{-3.5}^{+5.6}$ & $1.5 / 6$ \\
\hline $\mathrm{J} 2256-1024$ & 150 & $1.8_{-0.6}^{+0.3}$ & $4.6_{-1.6}^{+2.3}$ & 30.3 & 5 & $2.4_{-0.9}^{+1.0}$ & $3.2_{-1.6}^{+2.6}$ & $2.0 / 6$ \\
\hline
\end{tabular}

Note. - Spectral properties of the five Fermi-associated radio MSPs, including the temperature $(\mathrm{kT})$, power-law index $(\Gamma)$, the measured absorbed flux $\left(\mathrm{F}_{x}\right)$, the logarithm of the $0.3-8 \mathrm{keV}$ luminosity $\left(\log _{10} \mathrm{~L}_{\mathrm{x}}\right)$, the $0.3-8 \mathrm{keV}$ efficiency $(\epsilon)$, and the ratio of the $\chi^{2}$ value to the degrees of freedom (DOF) for each fit. The very low $\chi^{2}$ values obtained suggest the fits to be overdetermined. Also included for the combined fit are the contributions to the unabsorbed flux from each component. All fits were performed using Chandra's fitting package, Sherpa. All five sources were fitted with three separate models: a power-law model, a blackbody model, and a combined model with both power-law and blackbody components. The results of all three fits are shown. For the combined fits, values without errors were held constant, as was the temperature for each source $(150 \mathrm{eV})$. 
Lightcurves were then determined for each source using counts in the 0.3 to $8 \mathrm{keV}$ range, as Chandra has very little effective area outside of that range. The number of background-subtracted counts detected for each source ranged from 43 to 141 (Table 4.1). Each lightcurve was binned such that each bin represents one tenth of the observation, so that all bins have equal exposure. For these lightcurves, an orbital phase of 0.25 corresponds to the superior conjunction of the system. These lightcurves were then compared to uniform distributions using the $\chi^{2}$ test and Kolmogorov-Smirnov (K-S) test (Press et al., 1989) to determine their orbital variability. We have left these lightcurves unfolded to show the consistency of the shape from orbit-to-orbit.

Spectra were then analyzed using Chandra's spectral fitting platform, Sherpa. The data were binned with 5 bins between 0.3 and $2 \mathrm{keV}$ and 4 bins between 2 and 8 $\mathrm{keV}$. Bins in the 0.3 to $2 \mathrm{keV}$ energy range are of equal width $(0.34 \mathrm{keV})$, as are bins in the 2 to $8 \mathrm{keV}$ energy range $(1.5 \mathrm{keV})$. This binning scheme was used in order to differentiate thermal emission (which we expect below $2 \mathrm{keV}$ ) and non-thermal emission (which we expect above $2 \mathrm{keV}$ ), which requires multiple bins above $2 \mathrm{keV}$. The data were then fitted over energies between 0.3 and $8 \mathrm{keV}$. Due to the small number of background-subtracted counts, we fixed nH, the neutral Hydrogen column density along the line of sight to the source at a constant value set by the dispersion measure, assuming 10 free electrons per neutral Hydrogen atom as is motivated by He et al. (2013). The resulting column densities are listed in Table 4.1.

Comparing the values to the total Galactic nH as estimated using the $H E A S A R C$ nH tool (based on the maps of Kalberla et al. (2005); Dickey \& Lockman (1990)), we 
found these values to be consistent. We fit each source with three separate models: a power-law model, a blackbody model, and a combined model with both powerlaw and blackbody components. Due to the low number of background-subtracted counts for PSRs J0023+0923 and J1810+1744, temperature and power-law index (Г) were held constant at $150 \mathrm{eV}$ and 1.5 , respectively, for their combined fits. These values are consistent with X-ray blackbody temperatures typically seen for millisecond pulsars (Bogdanov, 2008; Bogdanov et al., 2007; Zhang \& Cheng, 2003) and power-law indices typically seen for non-thermal neutron star emission Bogdanov et al. (2005, 2011). ${ }^{3}$ Since PSRs J1124-3653, J2215+5135, and J2256-1024 all had higher count rates, we kept their temperatures fixed at $150 \mathrm{eV}$, but let $\Gamma$ vary. Also due to the low number of counts, all fits were done using cstat, which is Sherpa's equivalent to XSPEC's Cash statistic.

\subsection{Results}

\section{PSR J0023+0923}

The lightcurve appears uniform (within $1 \sigma$ errors), and, according to the K-S test, has a probability of 0.99 of being drawn from a uniform distribution. This is consistent with this pulsar showing no radio eclipse, even at the relatively low observing frequency of $350 \mathrm{MHz}$. A two-dimensional K-S test yields the probability of being a point source of 0.99 in the $\mathrm{x}$-direction and 0.31 in the $\mathrm{y}$-direction. Therefore, we conclude that there is no evidence for extended emission. There is no detected

\footnotetext{
${ }^{3}$ Also see http://astro.phys.wvu.edu/XrayMSPs for a list of millisecond pulsars observed in $\mathrm{X}$-Rays and their fit parameters.
} 

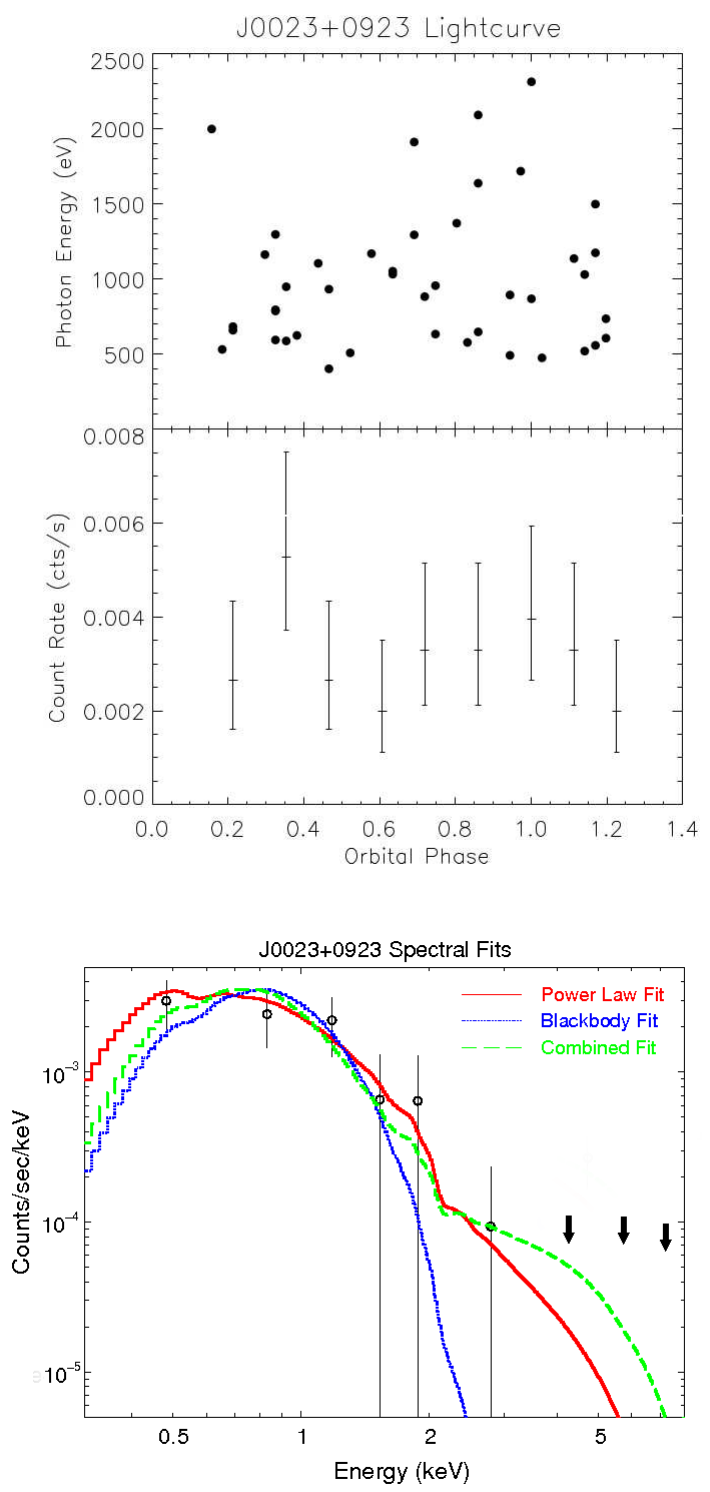

Figure 4.1: Count rate versus orbital phase and spectral fits for PSR J0023+0923. Where applicable, radio eclipse start and stop times are shown as dotted lines and upper limits on the count rates of empty bins are shown as arrows. All error bars correspond to $1 \sigma$ errors. Fit parameters are summarized in Table 4.2. 
emission above $2.5 \mathrm{keV}$, and so we effectively had only 6 bins (4 DOF) with which to fit, the very low $\chi^{2}$ values obtained suggest the fits to be overdetermined. While formally the power-law fit is slightly better than the blackbody fit, the lack of high energy counts and the steep power-law index $(\Gamma \sim 3)$ of the power-law fit, and the reasonable temperature obtained from the blackbody fit all suggest the emission is predominantly thermal.

\section{PSR J1124-3653}

The lightcurve shows marginal orbital variability as evidenced by the K-S test, which yields a probability of 0.10 of being drawn from a uniform distribution. Although the lowest count rate occurs at an orbital phase of 0 , there is a local minimum near an orbital phase of 0.25 (superior conjunction), which coincides with the radio eclipse phase (shown in Figure 4.2). Aside from these minima, the lightcurve is constant within the $1 \sigma$ error bars. A two-dimensional K-S test yields the probability of being a point source of 0.10 in the $\mathrm{x}$-direction and 0.65 in the $\mathrm{y}$-direction. Although the probability of the source being drawn from the same distribution as the PSF in the $\mathrm{x}$-direction appears low, we note that the source is actually narrower than the PSF in the $\mathrm{x}$-direction (consistent with Poisson variations) and therefore conclude that there is no evidence for extended emission for PSR J1124-3653. The spectrum is well fit by a simple power law while a blackbody fit is formally unacceptable and results in a very high temperature.

Since the count rate is higher than for PSR J0023+0923, we fix temperature 

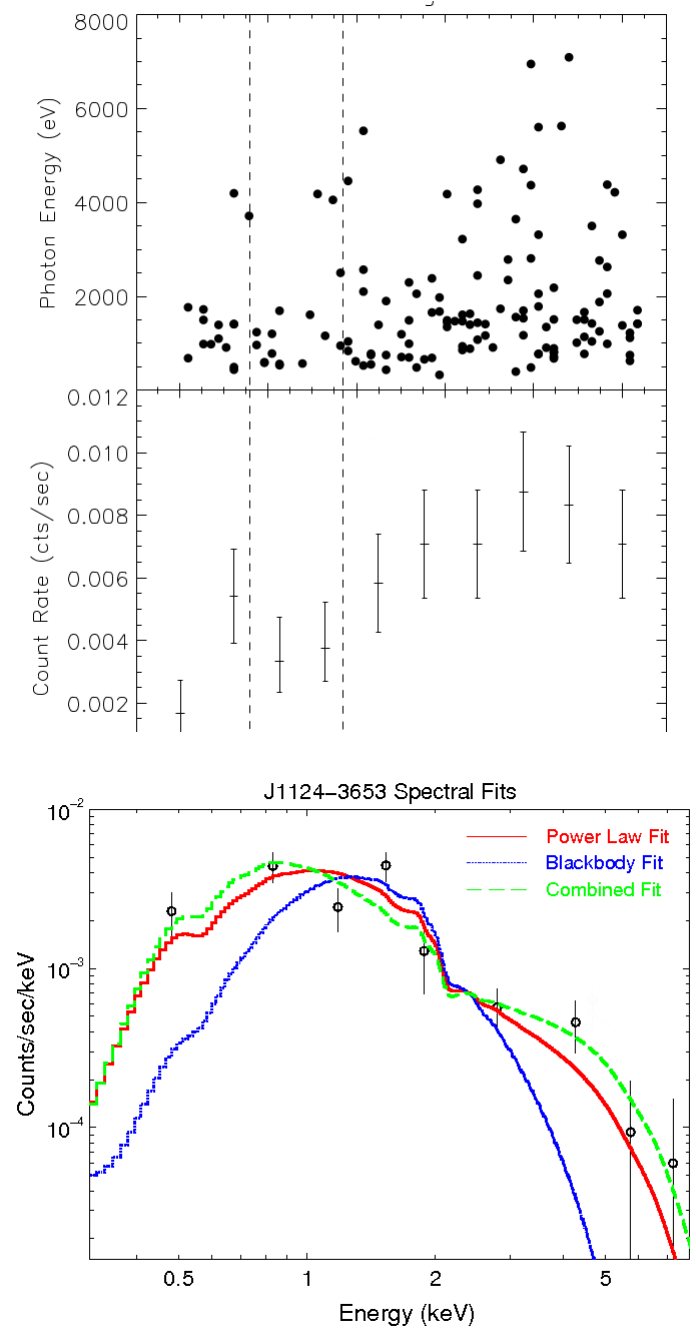

Figure 4.2: Same as Figure 4.1 for PSR J1124-3653. 
but let $\Gamma$ vary for the combined fit. Most of the flux from the combined fit is assigned to the power-law component.

\section{PSR J1810+1744}

The lightcurve does not look obviously uniform and the K-S test gives this lightcurve a probability of 0.43 of being drawn from a uniform distribution. The variation in the lightcurve is very broad, covering most of the orbit, making it unlikely that the orbital variation can be attributed to eclipsing of the intrabinary shock emission by the companion. The soft lightcurve $(0.3-2 \mathrm{keV})$ by itself does not show strong evidence for orbital variability. The two-dimensional K-S test yields the probability of being a point source of 0.23 in both directions. Therefore, we conclude that there is no clear evidence for extended emission. We again fix temperature and $\Gamma$ for the combined fit, with most of the flux from the combined fit coming from the power-law component.

\section{PSR J2215+5135}

The single redback in our sample has a lightcurve which is clearly not uniform and the K-S test confirms this by yielding a probability of 0.04 of being drawn from a uniform distribution. Both the hard and soft lightcurves include clear minima at the same orbital phase as the radio eclipse. A two-dimensional K-S test yields the probability of being a point source of 0.19 in the $\mathrm{x}$-direction and 0.27 in the y-direction. Therefore, we conclude that there is no strong evidence for extended 

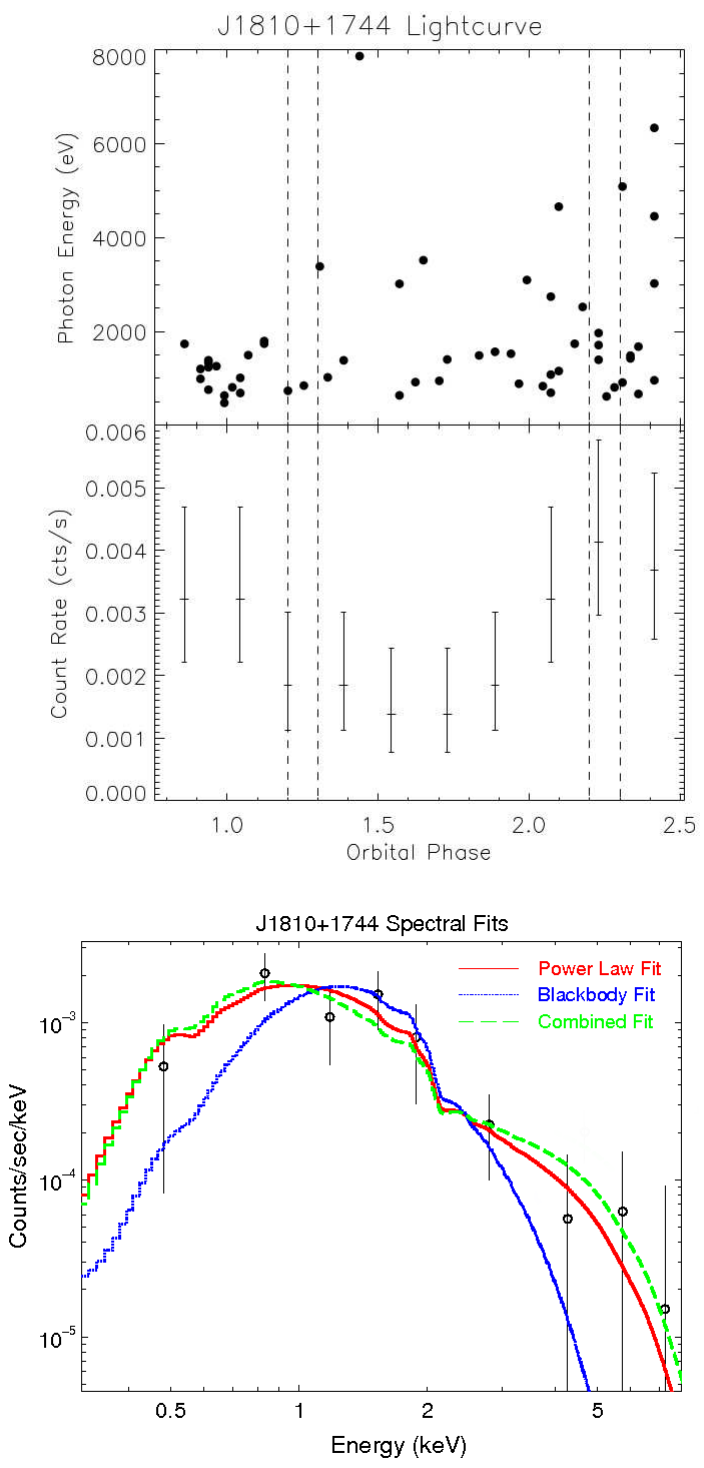

Figure 4.3: Same as Figure 4.1 for PSR J1810+1744. 

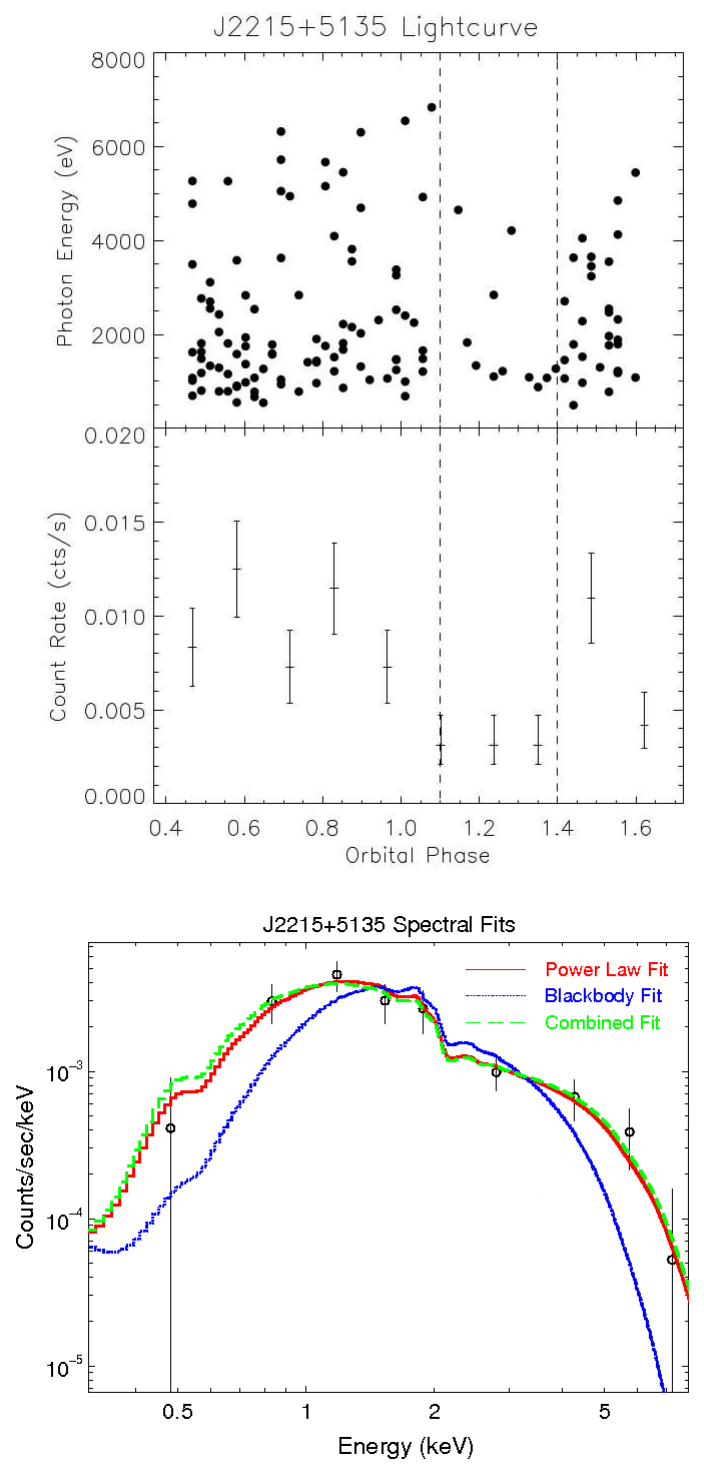

Figure 4.4: Same as Figure 4.1 for PSR J2215+5135. 
emission.

The spectrum is very hard, with a clear power-law tail. The blackbody fit resulted in a much higher $\chi^{2}$ value and an unacceptably high temperature. We fix temperature, but let $\Gamma$ vary for the combined fit. The flux from the combined fit is again dominated by the power-law component.

\section{PSR J2256-1024}

The lightcurve has clear minima near orbital phases of 0.25 and 1.25 and the K-S test gives this lightcurve a probability of $8.8 \times 10^{-3}$ of being drawn from a uniform distribution. Although the dip around 0.25 is pronounced, we only have a single coverage of the minimum. Although we do not see the same dip in the soft lightcurve, the hard lightcurve does seem to have dips at the same orbital phases that the general lightcurve has. The dips coincide with the measured radio eclipses. A two-dimensional K-S test yields the probability of being a point source of 0.96 in the $\mathrm{x}$-direction and 0.60 in the $\mathrm{y}$-direction. Therefore, we conclude that there is no evidence for extended emission.

Both the power-law and blackbody fits are acceptable, with a reasonable temperature and a somewhat steep spectral index. However, around $5 \%$ of the photons are above $4 \mathrm{keV}$, which, along with the orbital variability, suggests a significant power-law spectral component. We fix temperature, but let $\Gamma$ vary for the combined fit. The F-test prefers the combined fit over the power-law fit with a significance of 0.95 , and the flux from the combined fit is fairly evenly split between blackbody 

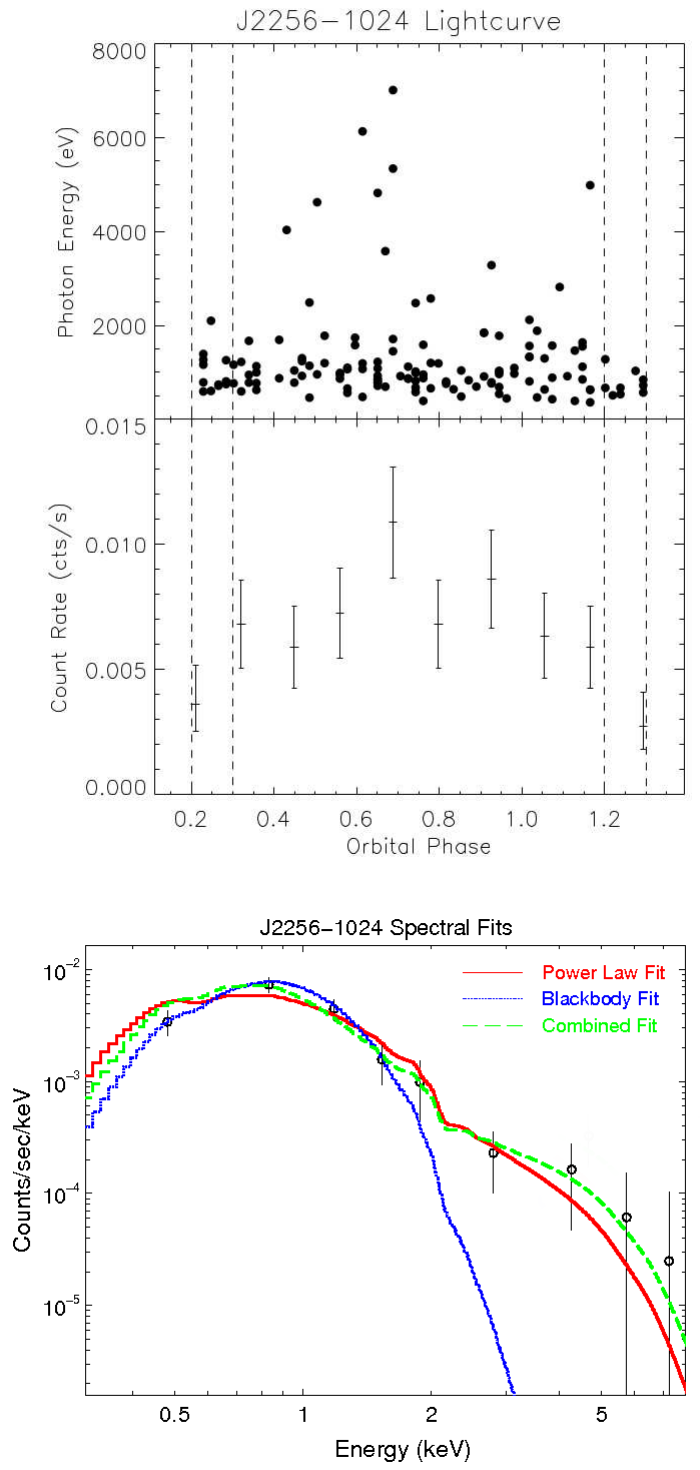

Figure 4.5: Same as Figure 4.1 for PSR J2256-1024. 
and power-law components.

\subsection{Discussion and Conclusions}

X-ray emission has been detected from roughly $50 \mathrm{MSPs}^{4}$. The emission can be described by either blackbody or power-law models and can originate from the neutron star surface (in the case of a blackbody model) or from the magnetosphere or an intrabinary shock (in the case of a power-law model).

We expect emission from the neutron star's surface and magnetosphere to be steady on timescales longer than the pulse period, and expect orbital modulation in the case of emission from an intrabinary shock. This modulation can be due to Doppler boosting of the flow within the shock, synchrotron beaming, or obscuration by the companion. In the first two cases, we would expect enhanced emission when the flow is coming towards us. Since there is only a weak outflow from the companion, we would expect a Mach cone pointed away from the pulsar with its head near the point on the companion star closest to the pulsar. For a nearly Roche-lobe filling companion, this would be near the L1 point. We might therefore expect a minimum near inferior conjunction (orbital phase 0.75), and, depending on inclination, a broad peak roughly centered around superior conjunction (orbital phase 0.25). However, the orbital motion would cause the Mach cone to be swept back, in which case a broad enhancement after superior conjunction may result. The companion could

\footnotetext{
${ }^{4}$ See http://astro.phys.wvu.edu/XrayMSPs for a full list of sources and for parameters used to calculate the luminosities in Figure 4.6.
} 


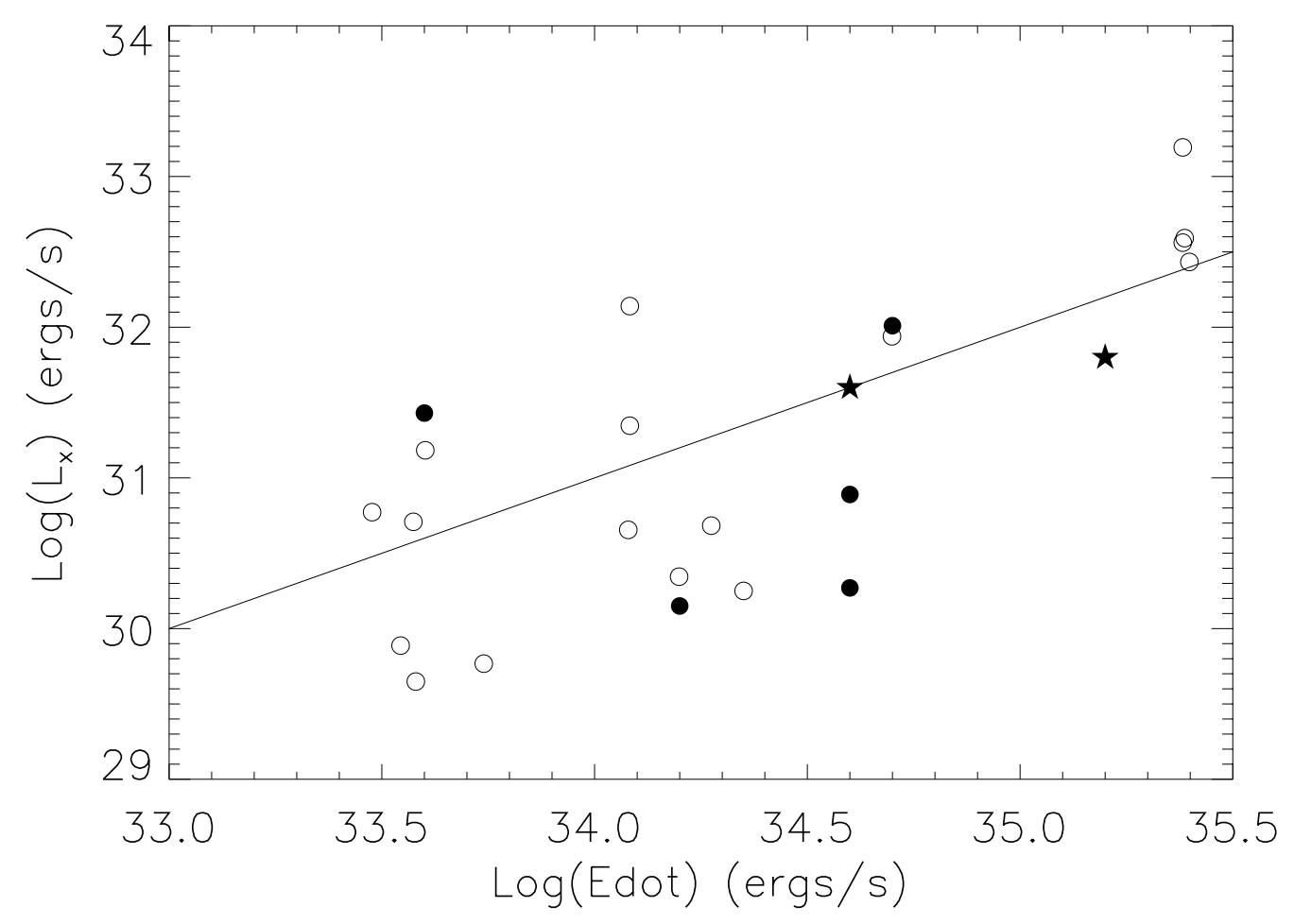

Figure 4.6: Luminosity versus spin-down energy loss rate $\dot{E}$ for 24 X-ray detected MSPs. PSRs J0023+0923, J1124-3653, J1810+1744, J2215+5135, and J2256-1024 are shown as filled circles. Open circles represent all other Galactic MSPs from which $\mathrm{X}$-ray emission has been detected. Spin-down energy loss rates were calculated using period derivatives that have been corrected for proper motion, and luminosities were calculated using distances derived from parallax measurements, where possible. PSRs J1023+0038 and B1957+20 are shown as stars for comparison. Luminosities of all sources were converted to equivalent luminosities in the $0.3-8.0 \mathrm{keV}$ range using WebPIMMS. The line represents $0.1 \%$ efficiency. 
also obscure part of the shock near superior conjunction, causing an X-ray dip. The duration and depth of the dip would depend on the ratio of the companion radius to the intrabinary separation, as well as the inclination angle of the system. With any of the above mechanisms, we would expect little if any change in the observed spectrum of the shock. Extended X-ray emission due to the pulsar wind shocking the interstellar medium has been detected from some MSP binary systems, and this would also be expected to be steady.

For emission arising from an intrabinary shock, the angle subtended on the pulsar's sky by the companion determines the fraction of the wind involved in the shock as well as affecting the X-ray light curve. If the companion is Roche-lobe filling, this fraction depends only on the masses of the binary components, which can be inferred from the timing modulo the inclination angle of the system. Modeling of the optical lightcurve of the companion can constrain both the inclination angle and the Roche-lobe filling factor of the companion. Breton et al. (2013) have made optical studies of all of our sources except for PSR J1124-3653, and compare them to PSR J1023+0038 and PSR B1957+20. All except PSR J0023+0923 and possibly PSR J2256-1024 seem to be nearly filling their Roche-lobes. The radius of the companion to PSR J0023+0923 may well be less than 2/3 its Roche-lobe radius, and the diameter subtends only $\sim 8^{\circ}$. PSR J2256-1024 subtends $\sim 11^{\circ}$, PSR J1810+1744 $\sim 15^{\circ}$, and PSR J2215+5135 $\sim 26^{\circ}$. All are viewed at moderate inclination angles $i \sim 45^{\circ}-70^{\circ}$. Although we do not have optical information on PSR J1124-3653, if it is nearly Roche-lobe filling as well, it would subtend $\sim 15^{\circ}$.

We detect X-ray emission from all five observed MSP binary systems (PSRs 
J0023+0923, J1124-3653, J1810+1744, J2215+5135, and J2256-1024). None of the pulsars show strong evidence for extended emission. In most cases, there is strong evidence for non-thermal emission, with power-law indices $\sim 1-2$ consistent with intrabinary shock emission, similar to what is seen in the modulated emission from PSR B1957+20 and PSR J1023+0038 (Bogdanov et al. (2005), Bogdanov et al. (2011)). While not well constrained given our low statistics, the ratio of non-thermal to thermal flux from our sample seems to roughly scale with the solid angle subtended by the companion. We also note that the X-ray luminosities for our sources are comparable to other pulsars with similar spin-down energy loss rates (see Figure 4.6 and Pavlov et al. 2007).

Two of the five pulsars show strong evidence for orbital modulation. PSR J2215+5135 shows an X-ray dip for roughly a quarter of the orbit around the radio eclipse. This is seen in both hard and soft lightcurves. Given the large angle the companion subtends on the pulsar sky, we should expect comparatively more intrabinary shock emission and a broader X-ray dip than the other sources, as well as even longer radio eclipses, even at high frequencies. Observations at $2 \mathrm{GHz}$ with the Green Bank Telescope show it to be eclipsed for roughly $1 / 3$ of the orbit . Similarly, we see a dip in the X-ray lightcurve around the radio eclipse for PSR J2256-1024. This dip is more pronounced in the hard lightcurve. We therefore conclude that the power-law spectral components for these two pulsars are primarily due to intrabinary shock emission. Another two of the five pulsars show marginal evidence for orbital variability, with broadly sinusoidal lightcurves. For PSR J1124-3653, the emission appears to peak around half an orbit after the radio eclipse, but comparing the 
beginning of the observation to the end also hints at orbit-to-orbit variability. This could be due to intrabinary shock emission, though a longer observation is necessary to further probe this. PSR J1810+1744 shows broad orbital variability around the orbit, with possible orbit-to-orbit variations. Given the often chaotic nature of wind shocks, this is only to be expected, as has been observed in both PSR B1957+20 and PSR J1023+0038.

The lightcurve of PSR J0023+0923 is nearly uniform, although due to the small number of counts, it is hard to make any concrete conclusion about variability. However, it also shows no evidence for radio eclipses and no evidence for emission above $2.5 \mathrm{keV}$. Given the companion's small angular extent and apparent under-filling of its Roche-lobe, meaning the surface material is much more strongly gravitationally bound than for the Roche-lobe filling systems, any contribution from shock emission is expected to be small.

We conclude that the emission from both PSRs J1124-3653 and J2256-1024 is likely due to a combination of thermal emission from the neutron star and powerlaw emission from an intrabinary shock. The orbital modulation and high ratio of power-law flux to blackbody flux seen in PSR J2215+5135's X-ray emission is consistent with being due primarily to an intrabinary shock. The temperatures and power-law indices derived are consistent with previous fits to neutron star spectra. For all three of these pulsars, a small magnetospheric contribution is also possible. Further X-ray observations with better timing resolution are necessary to determine this. Given the small number of counts for PSRs J0023+0923 and J1810+1744, it is difficult to make conclusions on the origin of the X-ray emission. However, given the 
emission from PSR J0023+0923 seems likely to be predominantly thermal, it is likely we are only seeing emission from the pulsar itself, with essentially no contribution from a shock.

The small number of photons detected from all of these sources prohibits a more detailed study or detailed geometrical modeling. However, in all cases the emission is dominated by an unresolved source, and likely comes from within the system with little or no contribution from an extended wind nebula. Therefore, future studies covering multiple orbits with any of the current imaging X-ray telescopes are highly desirable. 


\section{Chapter 5}

\section{X-Ray Observations of Redback Pulsars}

To be submitted to ApJ with co-authors Torres, R., Roberts, M. S. E., and McLaughlin, M. A.

\subsection{Introduction}

Of all the ways that pulsars can be characterized, perhaps the most useful, and certainly the most ubiquitous, is to characterize them by their spin period. This natually splits the pulsar population into two groups: "normal" or canonical pulsars, which have spin periods $\gtrsim 0.03$ seconds, and millisecond pulsars (MSPs), which have spin periods $\lesssim 0.03$ seconds (Lorimer \& Kramer, 2005). The endeavor to understand how these two groups of pulsars are phenomenologically connected has resulted in the "recycling" theory of MSP formation, whereby the pulsar accretes material from its companion and in the process gains angular momentum, which serves to "spin up" the pulsar (Alpar et al., 1982).

This formation scenario assumes the existence of a companion, however, of the 347 MSPs currently known, 122 (or 35\%) do not have companions (Manchester et al., 2005) ${ }^{1}$. This means that either these isolated MSPs were subjected to a different formation scenario (an assertion which does not have strong evidence behind

\footnotetext{
${ }^{1}$ http://www.atnf.csiro.au/research/pulsar/psrcat
} 
it), or they have somehow lost their compnions by some process. One possible explanation is that the millisecond pulsar lost its companion through stellar interactions. Although this may be a viable explanation for a number of globular cluster pulsars (Verbunt \& Freire, 2014), it does not reasonably explain the 39 Galactic isolated $\mathrm{MSPs}^{2}$.

The most promising explanation for these pulsars is that after the recycling stage, the wind of the resulting MSP interacts with the companion star, ablating it (Alpar et al., 1982; Ruderman et al., 1989). Evidence for the veracity of this scenario includes the existence of binary pulsars in tight orbits around low-mass companions $\left(\mathrm{M}_{c, \min } \ll 0.1 M_{\odot}\right)$ that display radio eclipses (indicative of excess intrabinary material). These pulsars, called "black widow" pulsars, are believed to be pulsars that are currently in the process of ablating their companions, their small companion masses suggesting they are relatively far along in the ablation process.

The first of these black widow pulsars, PSR B1957+20, showed the aforementioned phenomenology, along with significant pulse delays surrounding the radio eclipse, further evidence of an abundance of intrabinary material (Fruchter et al., 1990). These seminal radio observations led to follow-up observations in X-rays, both with XMM-Newton (Huang \& Becker, 2007) and Chandra (Stappers et al., 2003; Huang et al., 2012). These observations showed not only a synchrotron source coincident with PSR B1957+20's position, but further analysis showed that this source displayed peculiar spatial and temporal characteristics. First, the emission proved to be quasi-sinusoidally modulated at the orbital period of the binary sys-

\footnotetext{
${ }^{2}$ See http://astro.phys.wvu.edu/GalacticMSPs/
} 
tem. Further, the emission showed a narrow local minimum at the pulsar's superior conjunction. These characteristics are not well described by occultation of X-rays emitted by the pulsar itself, but they rather suggest a much broader emission region closer to the companion star. This emission was therefore interpreted as resulting from the pulsar wind shocking the surface of the nearly Roche-lobe filling companion star (van Kerkwijk et al., 2011).

Another important piece of the MSP evolutionary timeline was uncovered with the discovery of PSR J1023+0038, a system comprised of a MSP in orbit around a companion with a mass of $\mathrm{M}_{c, \min } \sim 0.2 M_{\odot}$ (Archibald et al., 2009, 2010). This relatively large companion mass is incompatible with the description of black widow pusars, though PSR J1023+0038 has many other characteristics that do fit the black widow description. For example, in addition to its radio emission showing complete eclipses coincident with the pulsar's superior conjunction, its X-ray emission also shows a decrease in flux coincident with superior conjunction. Additionally, its Xray spectrum is not well described by a purely thermal model, necessitating the addition of a power law component which dominates the X-ray flux. Further, the flux from the power law component is reduced both in general and with respect to the thermal component at superior conjunction (Bogdanov et al., 2011). As the inclination of the system is not particularly high $\left(i \sim 46^{\circ}\right.$, see Archibald et al., 2009), it follows that the bulk of the X-ray emission is not coming from the pulsar itself. Rather, the emission region must be much closer to the companion star.

It is highly unlikely that the similarities this system shares with black widow pulsars is a coincidence, although the orders-of-magnitude higher companion mass 
do indicate that PSR J1023+0038 is a distinct type of object. The most likely explanation is that PSR J1023+0038 (and other sources like it) are closely related to black widows, but have not progressed nearly as far in their evolution. An attractive possibility is that these sources, called "redbacks", are pulsars that have just recently finished (or are in the process of finishing) their "recycling" phase, during which they accrete material from their companion and spin-up. This possibility is exceptionally well-evidenced by the fact that PSR J1023+0038 was, previous to its discovery as a pulsar binary system, believed to contain an accretion disk (Bond et al., 2002; Szkody et al., 2003).

We present the results from X-ray observations taken with $X M M$-Newton for three of these "redback" systems: PSR J2215+5135, which has shown evidence for orbitally modulated X-ray emission (Gentile et al., 2014), and PSRs J1622-0315 and J1908+2105.

\subsection{Observations and Analysis}

We observed PSRs J1622-0315, J1908+2105, and J2215+5135 for 20, 20, and 54 ks respectively, which represent 1.4, 1.6, and 3.6 orbits for each respective redback system. The data were taken using the "Full Frame" mode for both the MOS and PN detectors, which have time resolutions of $2.6 \mathrm{~s}$ and $73.4 \mathrm{~ms}$ respectively in this mode. The data were then reduced using XMM-Newton's Science Analysis System $(\mathrm{SAS})^{3}$ in conjunction with the most recent Calibration files.

\footnotetext{
${ }^{3}$ See https://www.cosmos.esa.int/web/xmm-newton/what-is-sas for more details regarding the SAS.
} 
In order to filter the data to exclude times that were affected by a flaring particle background, we first created a region consisting of the entire chip containing our source, excluding any point sources and chip edges. We then analyzed the lightcurve for this region, and defined good time intervals to be those times in which the emission from this region did not exceed the typical background emission. This was done for each detector for each source.

Radio timing measurements put tight constraints on each of the sources' positions, and each source showed a point source coincident with its corresponding radio timing position, thus circular source regions were chosen to be centered on the radio timing position with radii of $20^{\prime \prime 4}$. Background regions were then chosen to be an annular region extending an additional $20^{\prime \prime}$ from the edge of the source region. In some cases, the source was close enough to the edge of the chip so that the background region included part of an adjacent chip. In these instances, any part of the background region that included another chip or a chip edge was excluded.

These source and background regions were then used in conjunction with the filtered datasets to make lightcurves and spectra. In order to generate the lightcurves, events between 0.2 and $10 \mathrm{keV}$ in the source and background regions were selected and barycentered, thereby generating raw source and background lightcurves. These lightcurves were then corrected for a number of instrumental effects with XMM-Newton's epiclccorr command, which also performed the background subtraction. The background-subtracted lightcurves from all three detectors

\footnotetext{
${ }^{4}$ This was chosen to reflect XMM-Newton's point spread function. For details, see https://heasarc.nasa.gov/docs/xmm/uhb/onaxisxraypsf.html.
} 

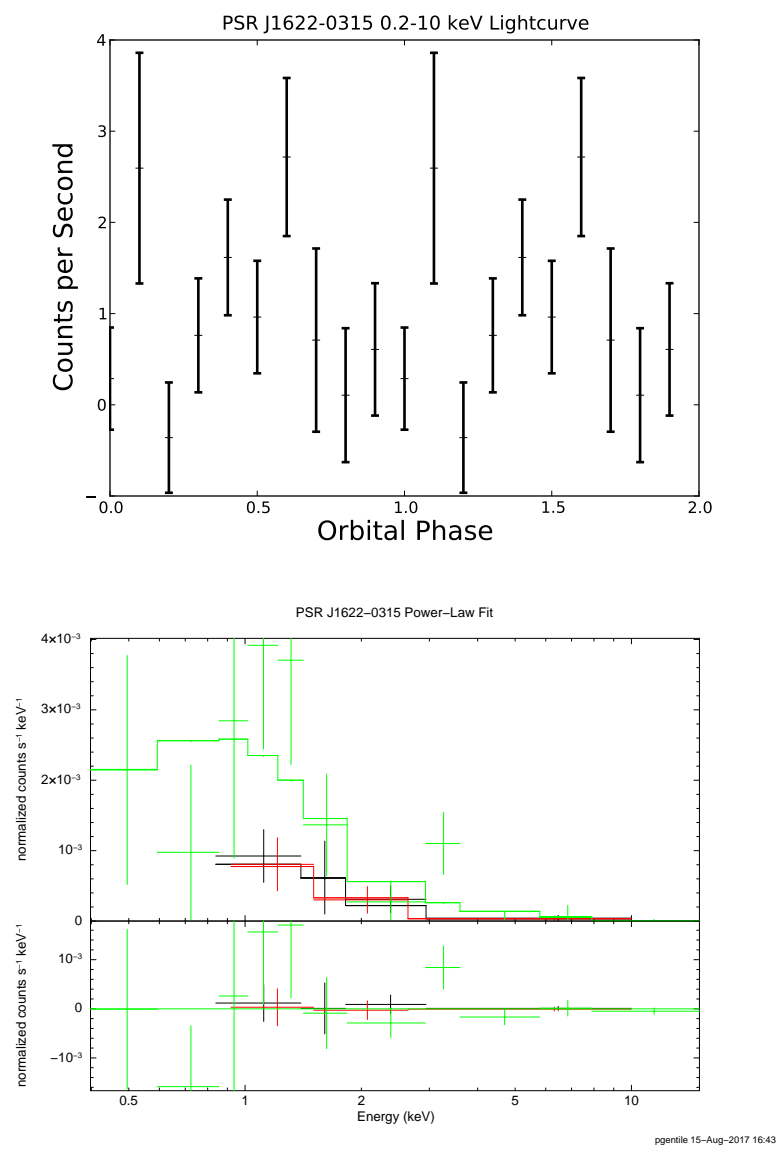

Figure 5.1: Count rate versus orbital phase and spectral fit for PSR J1622-0315. Black, red, and green spectral data points are taken from XMM-Newton's MOS1, MOS2, and PN detectors respectively, and the best fit model is overlaid in similarly colored lines. Error bars on both plots represent 1- $\sigma$ errors. Fit parameters are summarized in Table 5.2.

were then combined and folded with HEASARC's efold command. As in other analyses, an orbital phase of 0.25 corresponds to the superior conjunction of the system.

To generate spectra, we similarly used the source and background regions described above not only to select events from which to generate raw source and background spectra, but also to quantify areas for these regions that are corrected for bad pixels and chip gaps. These source and background spectra were then used 
to generate redistribution matrix files and ancillary response files.

These spectra could then be fit with xspec, however it was first necessary to determine a reasonable estimate of the neutral Hydrogen $(\mathrm{nH})$ column density along the line of sight to each source. Güver \& Özel (2009) find that the nH column density can be estimated given a measurement of the optical extinction $A_{V}$ along a given line of sight using the following expression:

$$
\mathrm{nH}=2.21 \times 10^{21} A_{V}
$$

where $\mathrm{nH}$ is in $\mathrm{cm}^{-2}$, and $A_{V}$ is in magnitudes. The optical extinction can be determined from the $B-V$ color excess, $E_{B-V}$, using the relation (Schultz \& Wiemer, 1975)

$$
A_{V}=3.14 E_{B-V}
$$

Using the Milky Way dust model found in Green et al. $(2015)^{5}$, we were able to determine $E_{B-V}$ values (and therefore initial $\mathrm{nH}$ values) for all of our sources. As the spectral fits were not able to tightly constrain the $\mathrm{nH}$ values, we held them fixed in all spectral fits.

We then fit each source with four spectral models: a power-law model, a blackbody model, a neutron star atmosphere (NSA) thermal emission model (described in Zavlin et al., 1996), and a combined blackbody/power-law model. A surface magnetic field strength of either $0,10^{12}$, or $10^{13} \mathrm{G}$ must be fixed to perform spec-

\footnotetext{
${ }^{5}$ This model can be queried for specific lines of sight using http://argonaut.skymaps.info/query
} 

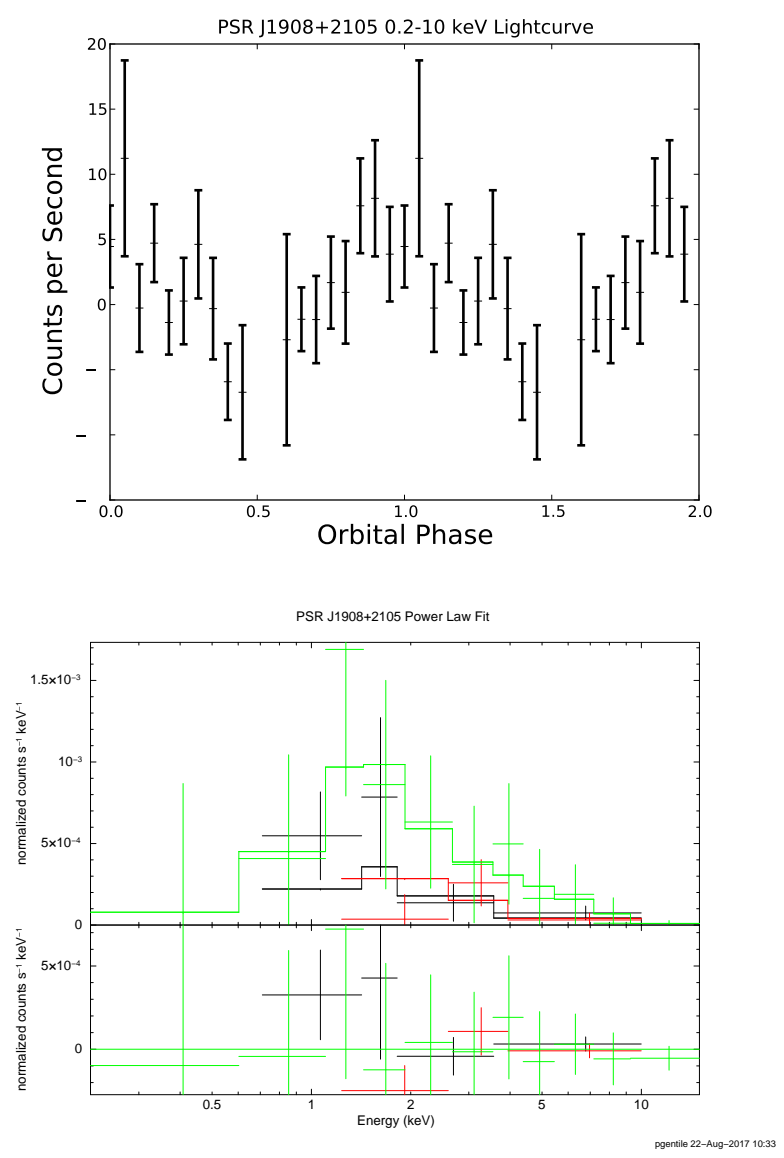

Figure 5.2: Same as Figure 5.1, but for PSR J1908+2105.

tral fits with the NSA model, and as MSPs typically have surface magnetic field strengths of $\sim 10^{8} \mathrm{G}$, we chose to perform the NSA spectral fits with a magnetic field strength of $0 \mathrm{G}$. Further, we fixed the neutron star mass and radius at $1.4 M_{\odot}$ and $10 \mathrm{~km}$ respectively. For the combined model, it was not always possible to fit for blackbody temperature or power-law index. We therefore fixed these values at 150 $\mathrm{eV}$ and 1.5 respectively where necessary (see Table 5.2 for details.) Additionally, since the unabsorbed fluxes from the fits performed with the combined model were dominated by the power-law component, it was not always possible to constrain the unabsorbed flux from the blackbody component. In these cases, we do not report a 
blackbody flux. In the cases where spectra could be fit with multiple models which each yielded physically reasonable best fit parameters, we determined the preferred model using the F-test, which is a model comparison test to determine which of two competing models best characterizes a data set (Press et al., 1989).

Table 5.1: Timing and X-ray Properties

\begin{tabular}{cccccccccc}
\hline $\begin{array}{c}\text { Name } \\
\text { PSR }\end{array}$ & $\begin{array}{c}P \\
(\mathrm{~ms})\end{array}$ & $\begin{array}{c}\log _{10} \dot{E} \\
\left(\mathrm{erg} \mathrm{s}^{-1}\right)\end{array}$ & $\begin{array}{c}\mathrm{DM} \\
\left(\mathrm{pc} \mathrm{cm}^{-3}\right)\end{array}$ & $\begin{array}{c}\mathrm{nH} \\
10^{20} \mathrm{~cm}^{-2}\end{array}$ & $\begin{array}{c}D \\
(\mathrm{kpc})\end{array}$ & $\begin{array}{c}P_{\text {orb }} \\
(\mathrm{hr})\end{array}$ & $\begin{array}{c}\mathrm{M}_{c, \min } \\
\left(\mathrm{M}_{\odot}\right)\end{array}$ & $\begin{array}{c}\mathrm{T}_{\text {obs }} \\
(\mathrm{ks})\end{array}$ & $\mathrm{MJD}_{\text {obs }}$ \\
\hline $\mathrm{J} 1622-0315$ & 3.85 & 33.9 & 21.4 & 15.0 & 1.1 & 3.9 & 0.10 & 20.9 & 57817 \\
$\mathrm{~J} 1908+2105$ & 2.56 & 34.5 & 61.9 & 51.0 & 3.2 & 3.5 & 0.06 & 20.9 & 58017 \\
$\mathrm{~J} 2215+5135$ & 2.61 & 34.7 & 69.2 & 21.4 & 3.0 & 4.2 & 0.22 & 53.8 & $57921-57922$ \\
\hline
\end{tabular}

Note. - Timing and X-ray properties of the three redbacks included in this analysis, including the pulsar spin period $(P)$, the logarithm of the spin-down energy loss rate $\left(\log _{10} \dot{E}\right)$, dispersion measure (DM), neutral Hydrogen column density along the line of sight to the source (nH), distance to the pulsar $(D)$, orbital period of the binary system $\left(P_{\text {orb }}\right)$, minimum companion mass $\left(\mathrm{M}_{\min }^{c}\right)$, total X-ray observation duration $\left(\mathrm{T}_{o b s}\right)$, and MJD of the observation $\left(\mathrm{MJD}_{o b s}\right)$. Due to the low background-subtracted count rates, $\mathrm{nH}$ is estimated from optical extinction (see Section 5.2) and held fixed for each source. Distances are estimated from the DM using the Cordes \& Lazio (2002) model for the Galactic electron density. 
Table 5.2: Spectral Fit Parameters

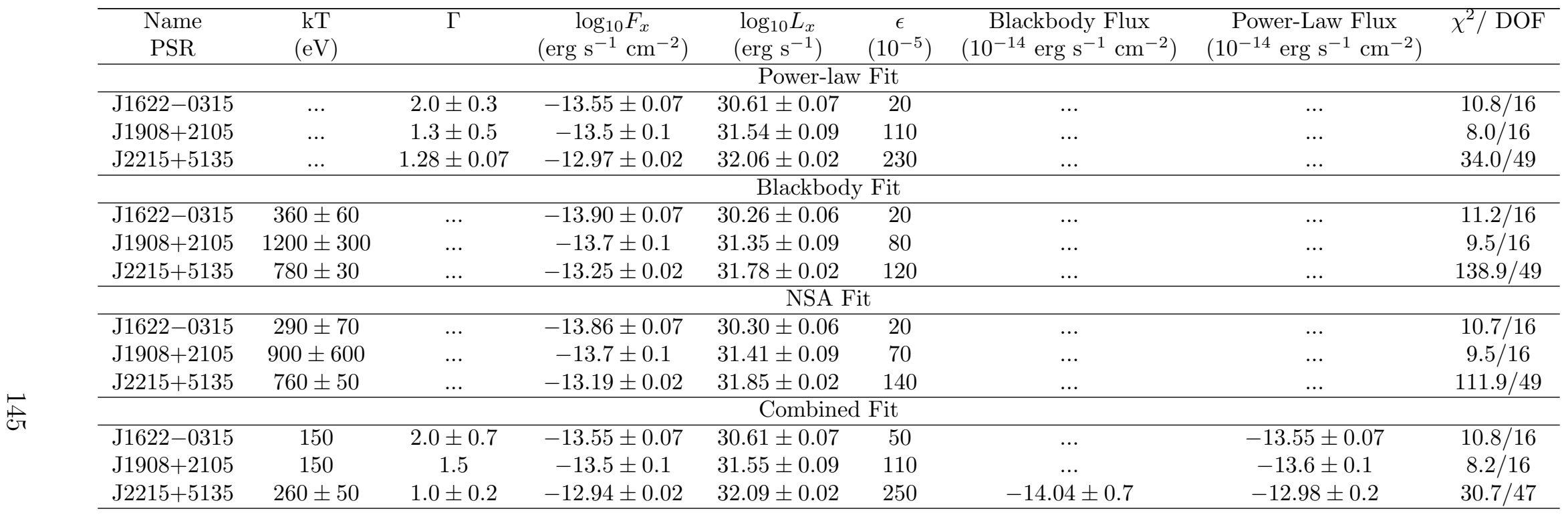

Note. - Spectral properties of the three redbacks included in this analysis, including the temperature $(\mathrm{kT})$, power-law index $(\Gamma)$, the unabsorbed flux $\left(\mathrm{F}_{x}\right)$, the logarithm of the $0.2-10 \mathrm{keV}$ luminosity $\left(\log _{10} \mathrm{~L}_{\mathrm{x}}\right)$, the $0.2-10 \mathrm{keV}$ efficiency $(\epsilon)$, and the ratio of the $\chi^{2}$ value to the degrees of freedom (DOF) for each fit. The very low $\chi^{2}$ values obtained suggest the fits to be overdetermined. Where possible, we also quote the contributions to the unabsorbed flux from each component of the combined blackbody/power-law fit. All fits were performed using XSPEC. All sources were fit with four separate models: a power-law model, a blackbody model, a neutron star atmosphere (NSA) model, and a combined model with both power-law and blackbody components. The results of all four fits are shown. For the combined fits, values without errors were held constant. 


\subsection{Results}

\subsubsection{PSR J1622-0315}

Although the lowest count rate in the lightcurve of PSR J1622-0315 does occur near superior conjunction, it does not show clear orbital variation overall. We note that the bin in the lightcurve with the lowest count rate has a negative value, which points to background subtraction issues, and therefore further observations with better data quality may more clearly establish the presence or absence of orbital variability.

PSR J1622-0315's spectrum shows hard X-ray emission that is not described well by thermal models with typical neutron star temperatures. We therefore reject the blackbody and NSA models. Because remaining models (the power-law model and the combined blackbody and power-law model) provide similar fits, and since the combined fit assumes a neutron star temperature of $150 \mathrm{eV}$, we prefer the powerlaw fit, as it makes fewer assumptions. This preference is further given credence by the fact that the combined fit indicates that almost all of the X-ray flux comes from the power-law component of the model.

\subsubsection{PSR J1908+2105}

The lightcurve of PSR J1908+2105 is incomplete, as excluding times affected by background flares meant that certain parts of the orbit were entirely removed from the analysis. From the remaining data, there does appear to be broad orbital variation, although we do not see a minimum near superior conjunction as 

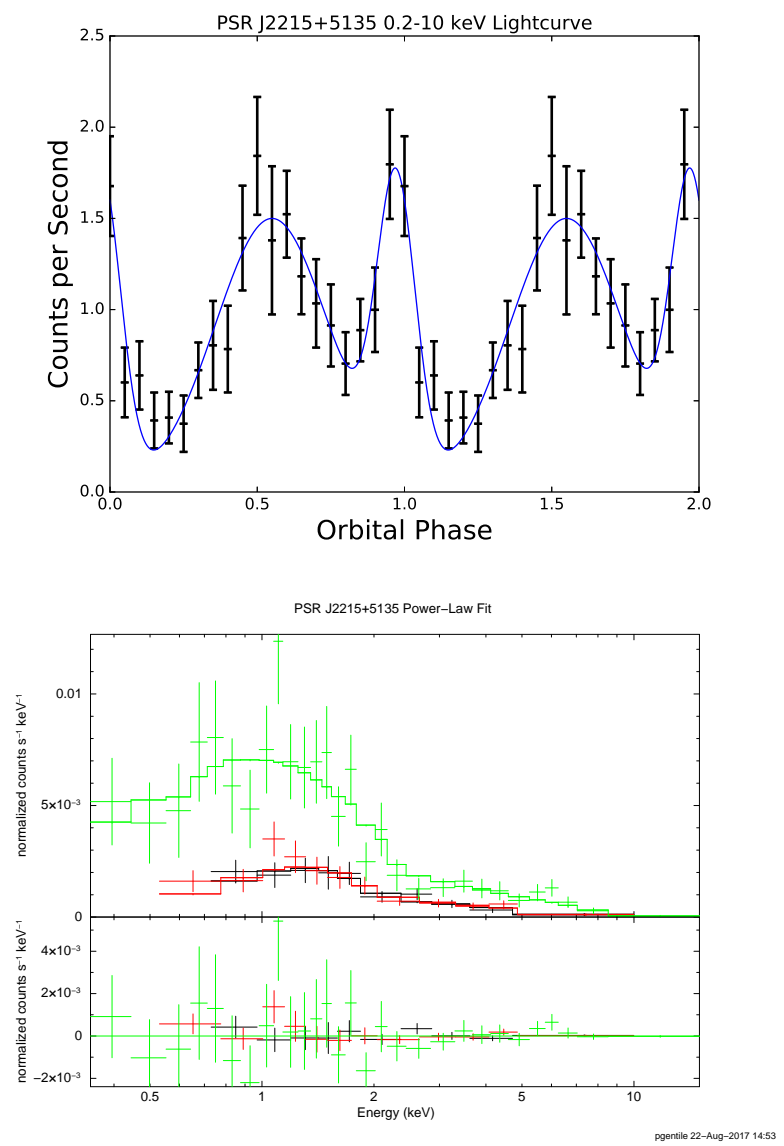

Figure 5.3: Same as Figure 5.1, but for PSR J2215+5135. The blue curve plotted on the lightcurve is the best fit model. See text for details.

may be expected from such a system. Further, we again note the existence of orbital phase bins with negative count rates, which, as with PSR J1622-0315, is indicative of background subtraction issues. Therefore, further observations would be needed to definitively characterize any orbital variability of PSR J1908+2105's X-ray lightcurve.

As with PSR J1622-0315, PSR J1908+2105's spectrum contains significant hard X-ray emission, and is consequently poorly described by a purely thermal model, as both the blackbody and NSA fits yield temperatures far higher than expected for neutron stars. We also note that even though we were not able to 
constrain temperature or power-law index in the combined fit, we were able to characterize the ratio of blackbody flux to power-law flux, the latter of which comprises nearly all of PSR J1908+2105's X-ray flux. We therefore prefer the power-law model for PSR J1908+2105's spectrum.

\subsubsection{PSR J2215+5135}

PSR J2215+5135's lightcurve is clearly non-uniform, and displays many characteristics consistent with X-ray observations of black widows and redbacks: a minimum near superior conjunction, and enhanced emission features away from superior conjunction. In the case of PSR J2215+5135, we see two clear peaks of emission, and a deep minimum in between. To further quantify these characteristics, we fit the lightcurve to a model consisting of two gaussians. From these fits, we see that the two components that make up the lightcurve are indeed well-described as gaussian. These components have comparable heights and are separated in phase by $\sim 0.42$ rotations. One stark difference between these components is their widths, with the wider of the components being $\sim 3$ times wider than the narrower component. It is also interesting to view this lightcurve model as the line of sight cuts through the orbital plane (see Figure 5.4). From this, it is easy to see the apparent asymmetry in the lightcurve.

As with the other sources we observed, PSR J2215+5135's spectrum is not fit well by a purely thermal model as the resultant fits return high $\chi^{2}$ values and unreasonably high temperatures. The combined fit returns a relatively high tem- 
perature, yet still yields a nonthermal flux that is more than an order of magnitude greater than the thermal flux. Though this fit appears to be reasonable, the F-test does not prefer it to the pure power-law fit.

Table 5.3: PSR J2215+5135 X-Ray Lightcurve Model Parameters

\begin{tabular}{cccc}
\hline Component & $\begin{array}{c}\text { Height } \\
(\text { Cts } / \mathrm{s})\end{array}$ & $\begin{array}{c}\text { Center } \\
\text { (Phase) }\end{array}$ & $\begin{array}{c}\text { FWHM } \\
\text { (Phase) }\end{array}$ \\
\hline 1 & $1.50 \pm 0.05$ & $0.550 \pm 0.008$ & $0.46 \pm 0.02$ \\
2 & $1.61 \pm 0.09$ & $0.972 \pm 0.004$ & $0.15 \pm 0.01$
\end{tabular}

Note. - Parameters resulting from fitting the X-Ray lightcurve of PSR J2215+5135 to a two Gaussian component model (See Figure 5.3).

\subsection{Discussion}

We have presented the X-ray detection (or re-detection) of three redback pulsars. Such detections in these kinds of systems are not unexpected. Indeed, pulsars possess temperatures high enough to produce blackbody emission in the X-rays, and the pulsar magnetosphere's outer gap (see Chapter 1) is expected to produce X-ray emission via synchrotron radiation. The former may be unmodulated or, in the presence of a heated polar cap, modulated, while the latter would similarly be modulated. These potential sources of modulated emission, however, both originate from regions that co-rotate with the pulsar, and therefore are expected to be modulated at the spin period of the $\operatorname{pulsar}^{6}$.

We have presented X-ray emission that is modulated on a much longer timescale:

\footnotetext{
${ }^{6}$ Further, the maximum time resolution available in the mode with which the data were taken (as described in Section 5.2) is much longer than our sources' spin periods, thus we would not be sensitive to these modulations in any case.
} 
at the period of the orbit of the binary system. This would indicate emission that is caused by an orbital interaction, and the characteristics of the orbit (an object with a powerful wind closely orbiting its companion) suggest the pulsar wind is interacting with the companion in an intrabinary shock.

The geometry of this shock is difficult to determine, although modeling the lightcurve of PSR J2215+5135 provides some insight. If the modulation of the X-ray emission was due to obscuration by the companion alone, we would expect an X-ray minimum near superior conjunction. While we do see such a minimum, we also note the existence of a second minimum near inferior conjunction.

We therefore conclude that the emission itself is inherently non-uniform. Characterizing that non-uniformity, though, would require a clearer description of the companion star (for example, optical measurements would be able to constrain how much of its Roche lobe it is filling and how efficient the pulsar wind/companion interaction is via companion star surface temperature fluctuation measurements), and deeper X-ray observations to more precisely characterize the X-ray lightcurves, especially for PSRs J1622-0315 and J1908+2105. 


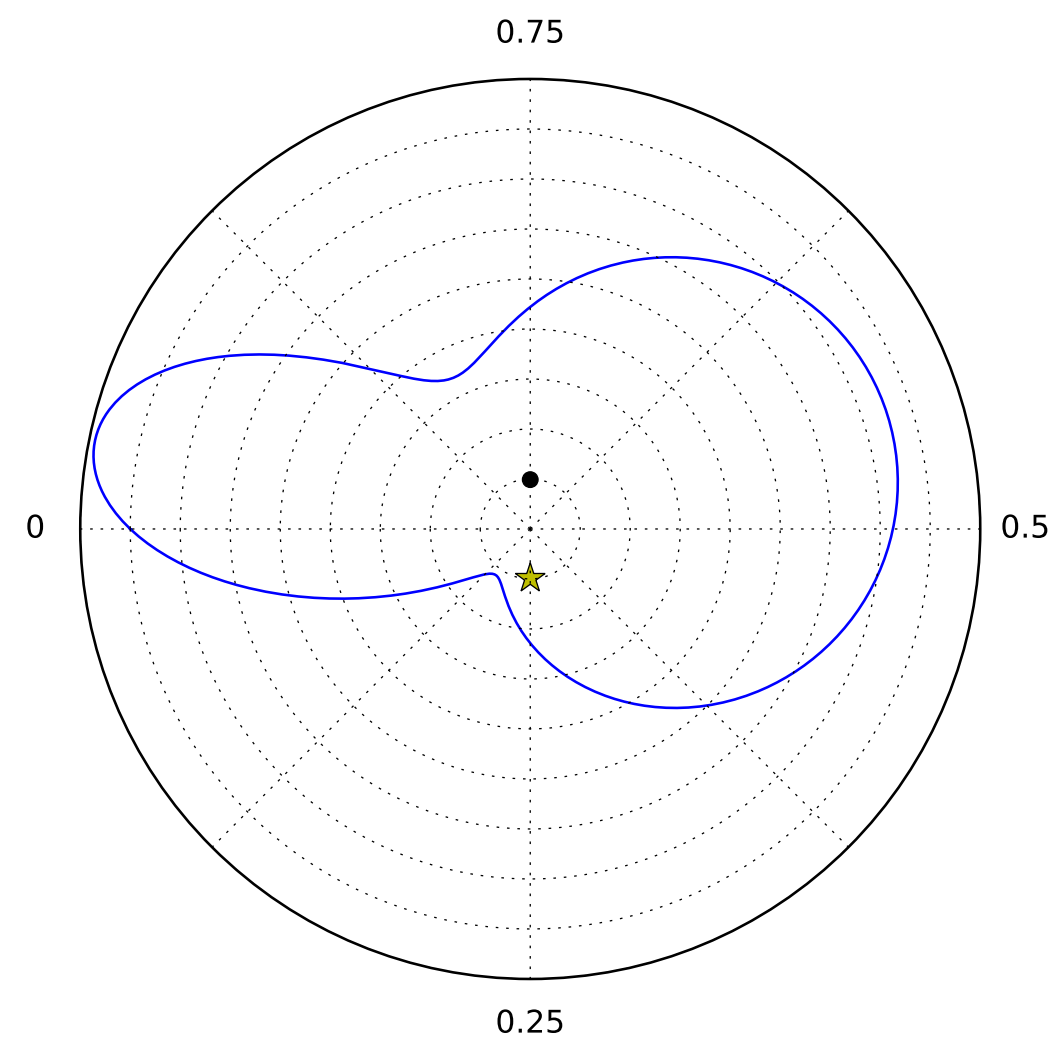

Figure 5.4: PSR J2215+5135's best fit model lightcurve plotted in polar coordinates. In this figure, the black dot represents the neutron star, the yellow star represents the companion star, the angle represents the orbital phase at which the observer would observe the system in a given configuration, and the blue curve represents the X-ray intensity the observer would detect according the model where the line being farther from the origin represents a stronger intensity. For example, superior conjunction occurs at an orbital phase of 0.25 when the companion star is between the neutron star and the observer, and we see the model predicts a low X-ray intensity, as reflected by the observed lightcurve shown in Figure 5.3. 


\section{Chapter 5}

\section{Conclusions}

\subsection{Pulsar Polarization and Receiver Modeling}

In Chapter 3, we described the implementation of a new method of polarimetric calibration, where short ( 30 minute) observations of "standard sources" could be used to generate a full receiver solution. To implement this method, we used two of the brightest and most well-studied pulsars (PSRs J1713+0747 and B1937+21) as "standard sources", which allowed us to calibrate the full NANOGrav dataset observed with the Arecibo telescope.

One result from this analysis was the polarization profiles themselves, a dataset which is the most sensitive to date. For many sources, this dataset included polarization profiles taken at multiple observing frequencies, providing insight as to the frequency evolution of the pulsar emission and therefore the mechanisms underlying it.

The sensitivity of this dataset also enabled the detection of faint profile features such as bridges of emission and profile components with intensities $\sim 100$ times smaller than the total intensity peak (called "microcomponents"). These detections can place tight constraints on the spin and emission geometries of the pulsars for which they have been detected. Further, the fact that these features were detected in the brightest sources raises the exciting possibility that they are not as rare 
as they may initially seem, and the advance of radio telescope sensitivities and pulsar observation techniques may bring with them the emergence of more previously undetectably faint profile features. The corollary to this is that we may eventually be able to detect MSPs using only their microcomponents, enabling the detection of all MSPs which have them. This therefore would make the development of radio telescopes the limiting factor to MSP detections, rather than characteristics inherent to the MSP, such as duty cycle.

We also were able to use the considerable bandwidth of Arecibo's L-wide and S-wide receivers to measure RMs via Faraday rotation. For the sources which were observed with both receivers, we were able to independently measure the RMs at each observing frequency and found such measurements to be broadly consistent. As the DMs of all these sources have been precisely determined, we were also able to use these measurements to determine the average Galactic magnetic field strength along the line of sight for each source. We found these values to be consistent with other, more detailed studies of the Galactic magnetic field.

Finally, since the calibration procedure employed in this work made it possible to generate a polarimetric response for Arecibo's L-wide and S-wide receivers at each epoch for which PSRs J1713+0747 and B1937+21 were observed, we were then able to determine that the polarimetric responses of these two receivers vary with time. This challenges the implicit assumption underlying conventional calibration strategies of a static polarimetric response and therefore indicates that such calibration strategies may not be adequate.

This analysis is far from complete, as it possesses a number of limitations both 
intrinsically and extrinsically. For example, the procedure assumes the profiles of the standard sources are perfectly calibrated. This is almost certainly not correct for our dataset, as the polarimetric responses that were used to initially calibrate the standard sources were made using data collected a number of days prior to the standard source data to be calibrated. Since the polarimetric response of the receiver changes with time, this will undoubtedly introduce some error into the process ${ }^{1}$.

Further, this work does not take into account the possibility that the parameters that govern the polarimetric response of a telescope change with respect to the parallactic angle on the sky. Since this has not been well-studied, its potential effects are unknown, however, if such a consideration is important, one might expect it to be especially so with a telescope such as Arecibo, where a different region of the dish is illuminated with respect to position on the sky.

Some of these limitations, such as the imperfect calibration of our standard sources, can be mitigated. Others, such as the possible variation of the parameters that govern the polarimetric response with parallactic angle, are shared by more conventional calibration strategies. As a result, using pulsars as standard sources may become the preferred method for polarimetric calibration for all astronomers observing sources at frequencies at which pulsars are bright.

This would have benefits for observers using the telescope, as it would allow a faster method for calibration, allowing observers who are unwilling or unable to spend observing time generating a polarimetric response using conventional meth-

\footnotetext{
${ }^{1}$ Though, as in Chapter 3, we stress the unlikeliness that these errors would be comparable to the errors that would be present if we did not use this procedure and instead assumed the polarimetric response of the receiver to be stable over time.
} 
ods to calibrate their data nonetheless. Additionally, with the adoption of using pulsars as standard sources would come the ability to characterize the behavior of the polarimetric response with time. Depending on this characterization, it may be possible to interpolate a new polarimetric response from ones that have been measured, enabling observers who could not observe a standard source ${ }^{2}$ to calibrate their data nevertheless.

It would also have benefits for the pulsar timing community, as it would mean that observers who may not have an interest in observing pulsars would now be helping to generate a potentially large amount of data on some of the brightest MSPs available. This would aid not only in the search for gravitational waves, but also in the investigation of the timing noise, DM variations, and flux variations.

\subsection{Spider Pulsars}

In Chapters 4 and 5, we presented X-ray detections of black widow and redback pulsars: fast-spinning MSPs thought to be ablating their companions, likely though the interaction of the pulsar wind with the companion. In addition to mass loss of the companion, this interaction is also able to produce intrabinary shock emission.

For a significant fraction of these sources, we detected hard, non-thermal Xrays. Though this could be explained through normal pulsar emission (that is, synchrotron emission emanating from the magnetospheric outer gap), the modulation of this emission with orbital phase proves this emission to be both non-thermal and non-magnetospheric. Thus, the likely explanation is the aforementioned intrabinary

\footnotetext{
${ }^{2}$ If, for example, the source had set.
} 
shock emission.

Interestingly, we first detected one source (PSR J2215+5135) with a relatively short observation with the Chandra X-Ray Observatory. These observations showed the most promising potential for deeper observations to provide more insight into the modulated X-ray emission. Those deeper observations were then performed with $X M M-N e w t o n$, where it was observed for over three full orbits of the binary system.

These deeper observations provided the desired increased sensitivity and with it, the anticipated orbital phenomenology. This phenomenology provided insight into the geometry of the modulated X-ray emission, which is inconsistent with a uniformly emitting region being occulted by the companion star.

X-ray observations of these exotic systems are exciting in their own right, but a motivation for them is to be able to characterize the mass loss of the system and in doing so, the process by which fast-spinning MSPs are formed. An essential component of this process is of course the companion star, therefore, this endeavor is aided by a deeper characterization of the companion star. While the future of our understanding of spider pulsars likely lies primarily with the discovery of more of them ${ }^{3}$, the systems presented here can provide more insight into the phenomenology of spider pulsar systems through characterization of their companions.

Additional radio observations of these systems could help us describe the intrabinary environment. Long-term timing campaigns could reveal changes in the companion's mass, although such a measurement would be difficult, as measuring companion mass is a difficult endeavor through normal pulsar timing, and the com-

\footnotetext{
${ }^{3}$ Which is excitingly non-hypothetical!
} 
plex nature of these systems makes them even more difficult to accurately time. A more straightforward analysis would be to measure the orbital dependence of the pulsar's dispersion measure, providing a measurement of the material in the binary system. Precise polarimetric calibration would also facilitate the measurement of RM variations with orbital phase, and thereby probe the magnetic field of the companion star.

An optical characterization of black widow and redback companions would be fruitful, as it would allow us to characterize the shock region in a different way: orbital variations of the optical properties of the companions would let us characterize the phenomenology of the companion star, and temperature differences between the day side (i.e., the side of the companion facing the pulsar) and the night side (i.e., the side of the companion facing away from the pulsar) would provide insight into the interaction between the pulsar wind and its companion, as this interaction would be responsible for such a difference.

In this thesis, I have described the implementation of a new calibration technique, the detection of pulse microcomponents, and the phenomenology of sider pulsar systems at X-ray energies. The next ten years are likely to see the implementation of this calibration scheme, or one like it, at many observatories performing radio pulsar observations. In that time, searches for pulsars are likely to uncover many more of these spider systems, the study of which will help characterize the mechanisms of their mass loss, including the overall companion mass loss rate. This will allow us to constrain the binary MSP to isolated MSP evolution timescale and can even help to constrain the upper limit on the pulsar recycling process. Further, 
as radio telescope technology improves, the existence of pulse microcomponents means pulsar searches will be able to detect MSPs even if their brightest emission beams do not cross our line of sight. Thus newly discovered MSPs will not necessarily be found farther away in the galaxy but may instead be discovered relatively nearby, creating the possibility of detecting nearby exotic systems like spider pulsar systems 
Appendix A

Polarization Profiles of the NANOGrav Arecibo Dataset 


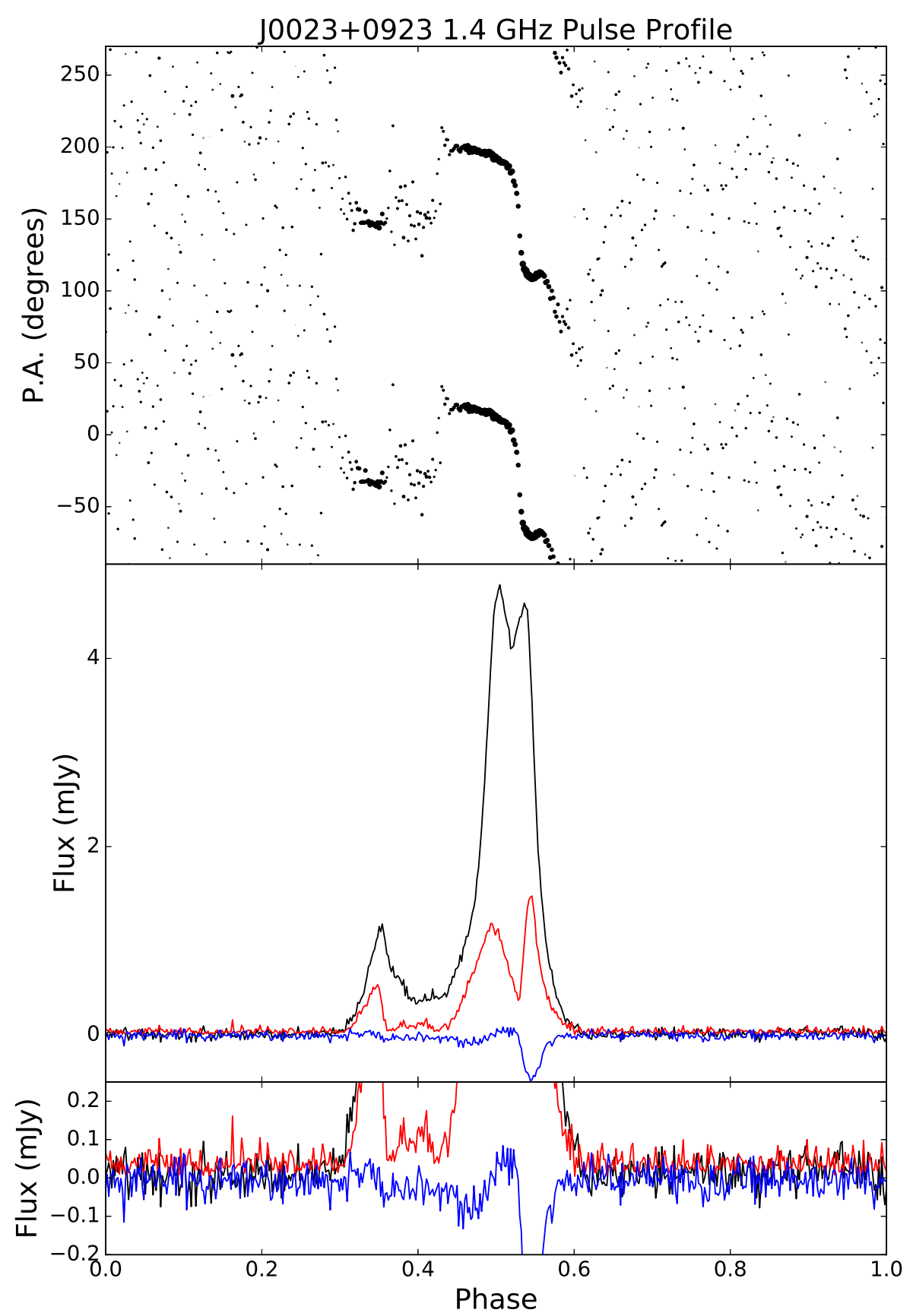

Figure A.1: Polarization profile of PSR J0023+0923 at 1.4 GHz. The top panel shows Position Angle (P.A.) in degrees, which is plotted twice for clarity. The middle panel shows the full polarization profile, that is, intensity versus pulsar spin phase. The profile is roughly aligned with the center of the on-pulse region. Total intensity is plotted in black, linear polarization in red and circular polarization in blue. The bottom panel is the same as the middle panel, except zoomed vertically to show any possible microcomponents in more detail. All panels are phase-aligned. 


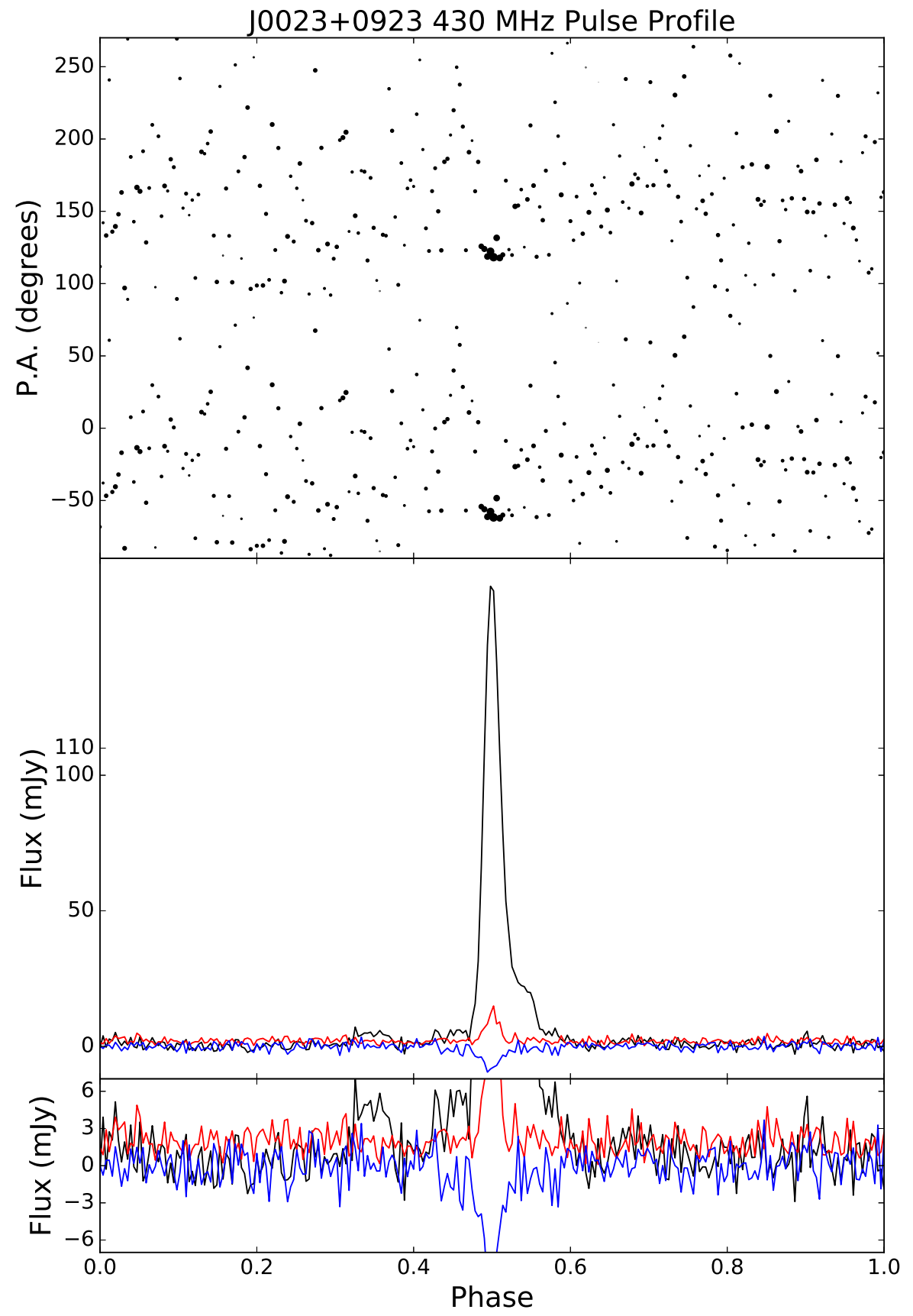

Figure A.2: Same as Figure A.1, for PSR J0023+0923 at 430 MHz. 


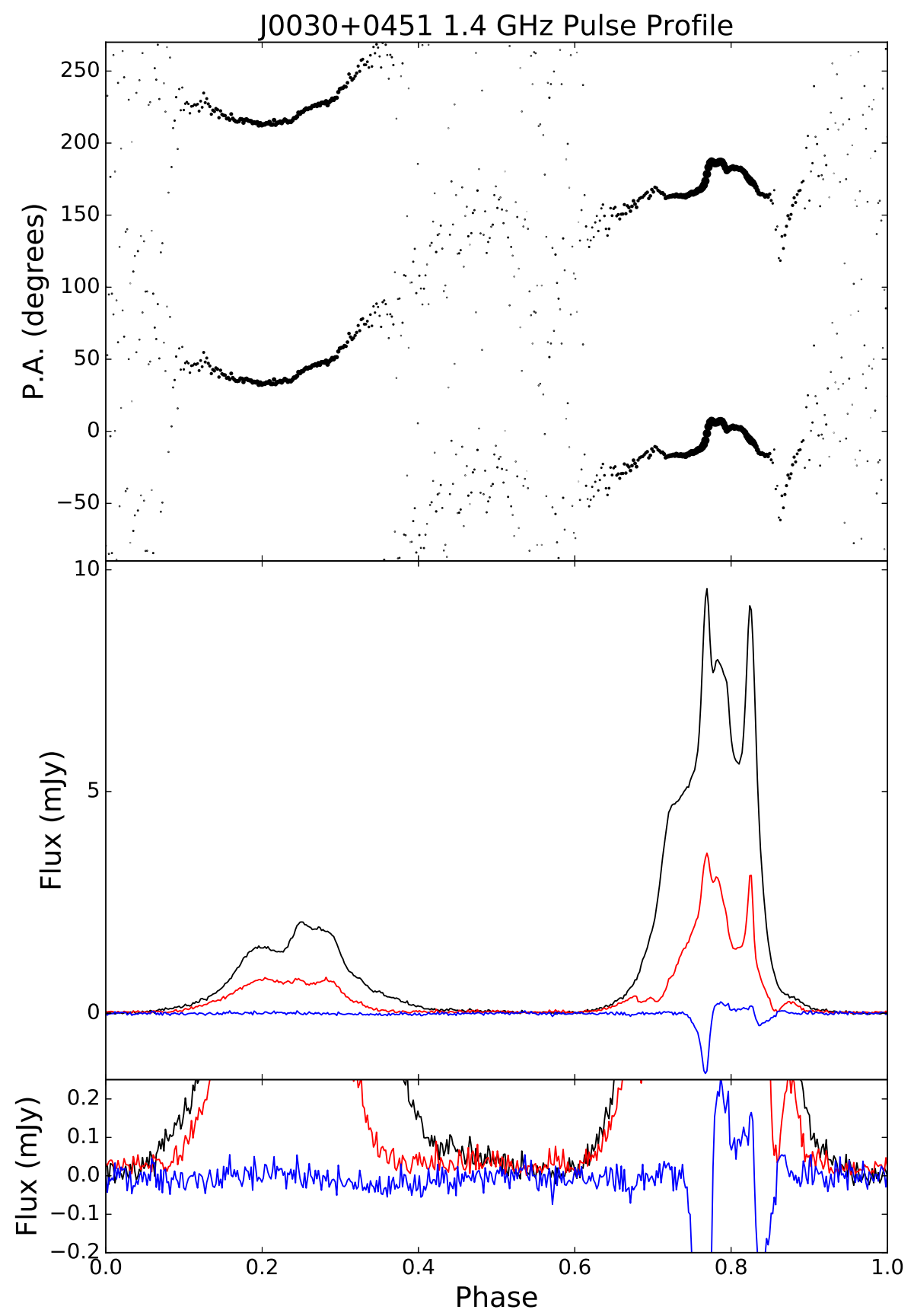

Figure A.3: Same as Figure A.1, for PSR J0030+0451 at 1.4 GHz. 


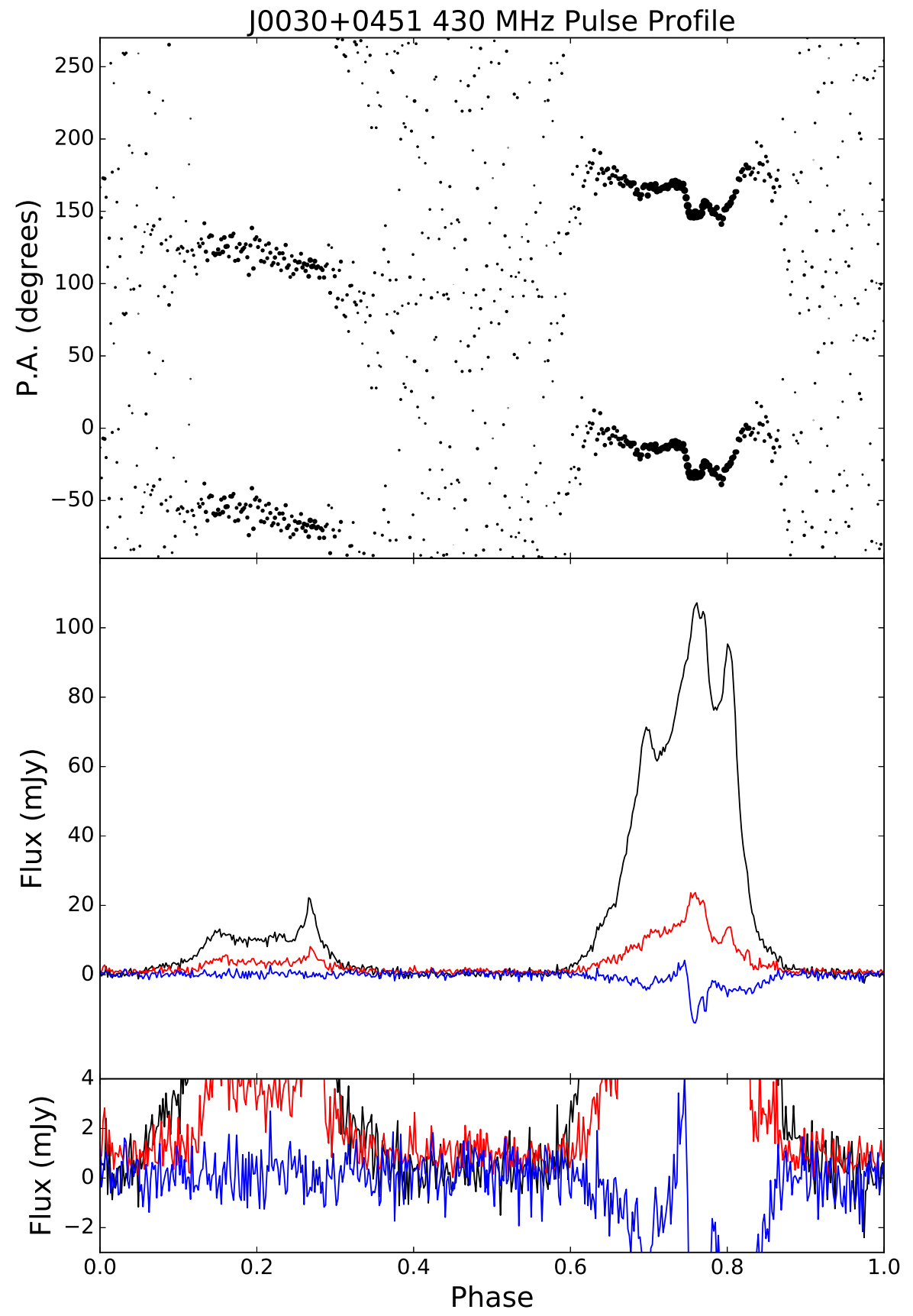

Figure A.4: Same as Figure A.1, for PSR J0030+0451 at $430 \mathrm{MHz}$. 


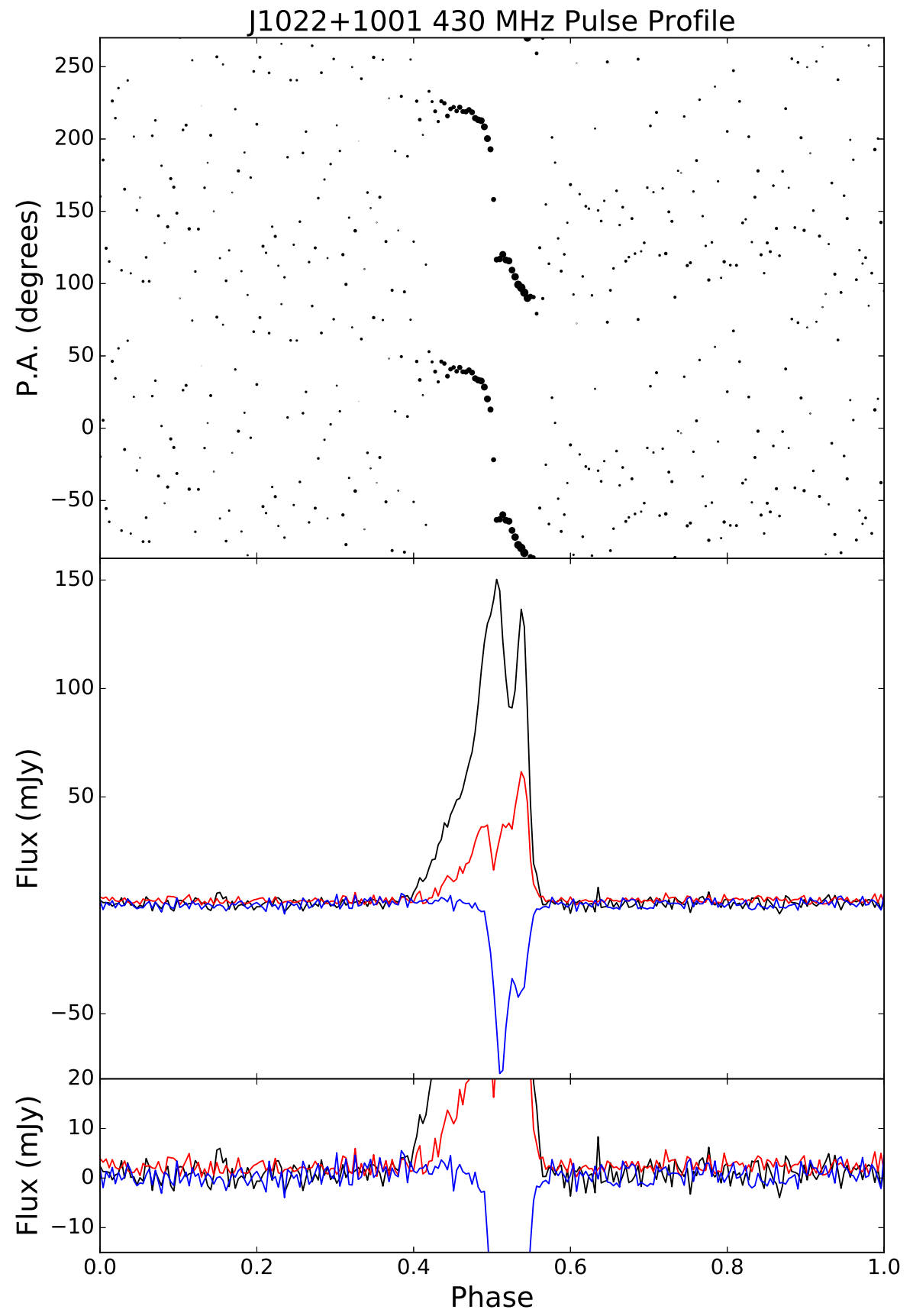

Figure A.5: Same as Figure A.1, for PSR J1022+1001 at $430 \mathrm{MHz}$. 


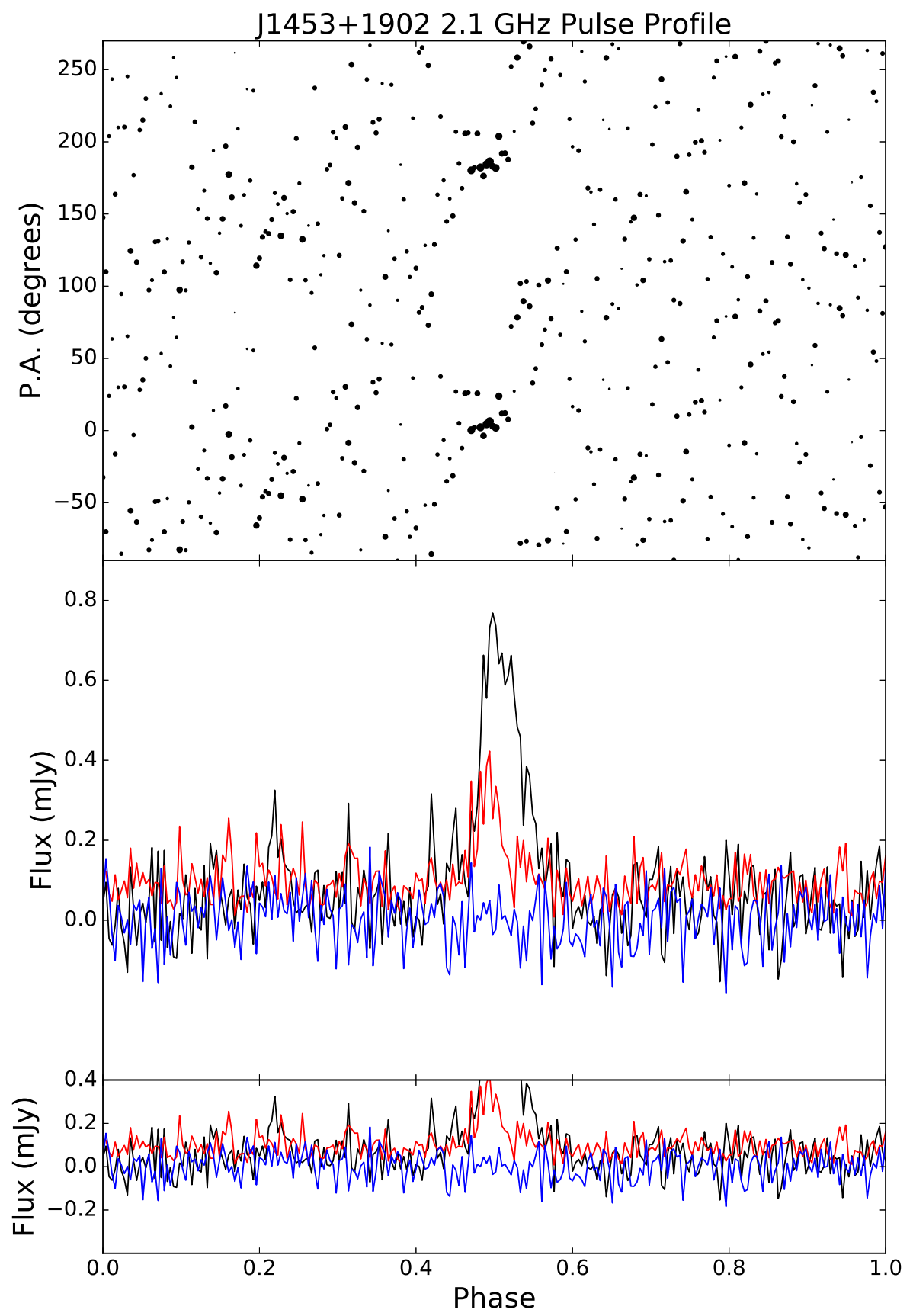

Figure A.6: Same as Figure A.1, for PSR J1453+1902 at 2.1 GHz. 


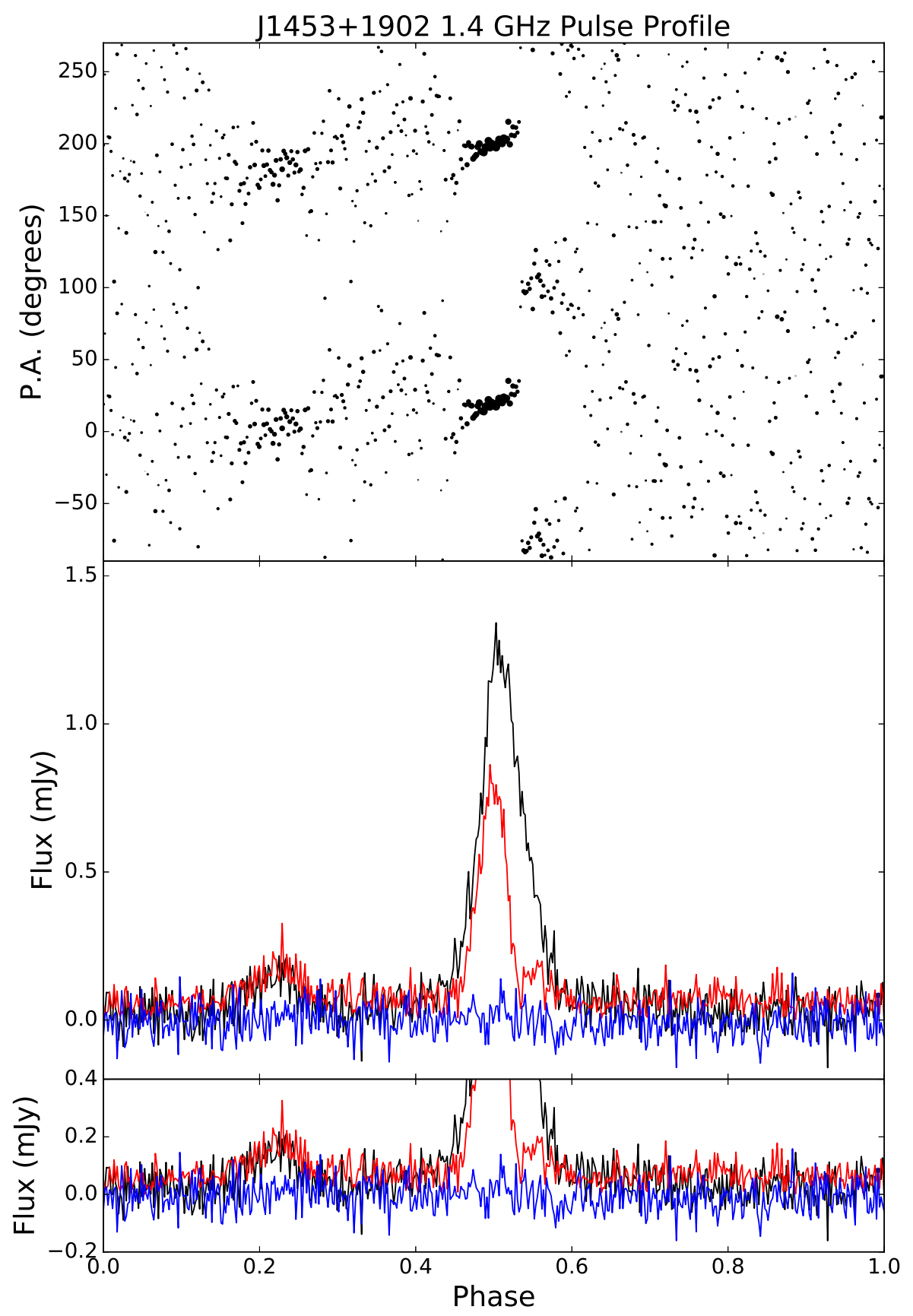

Figure A.7: Same as Figure A.1, for PSR J1453+1902 at 1.4 GHz. 


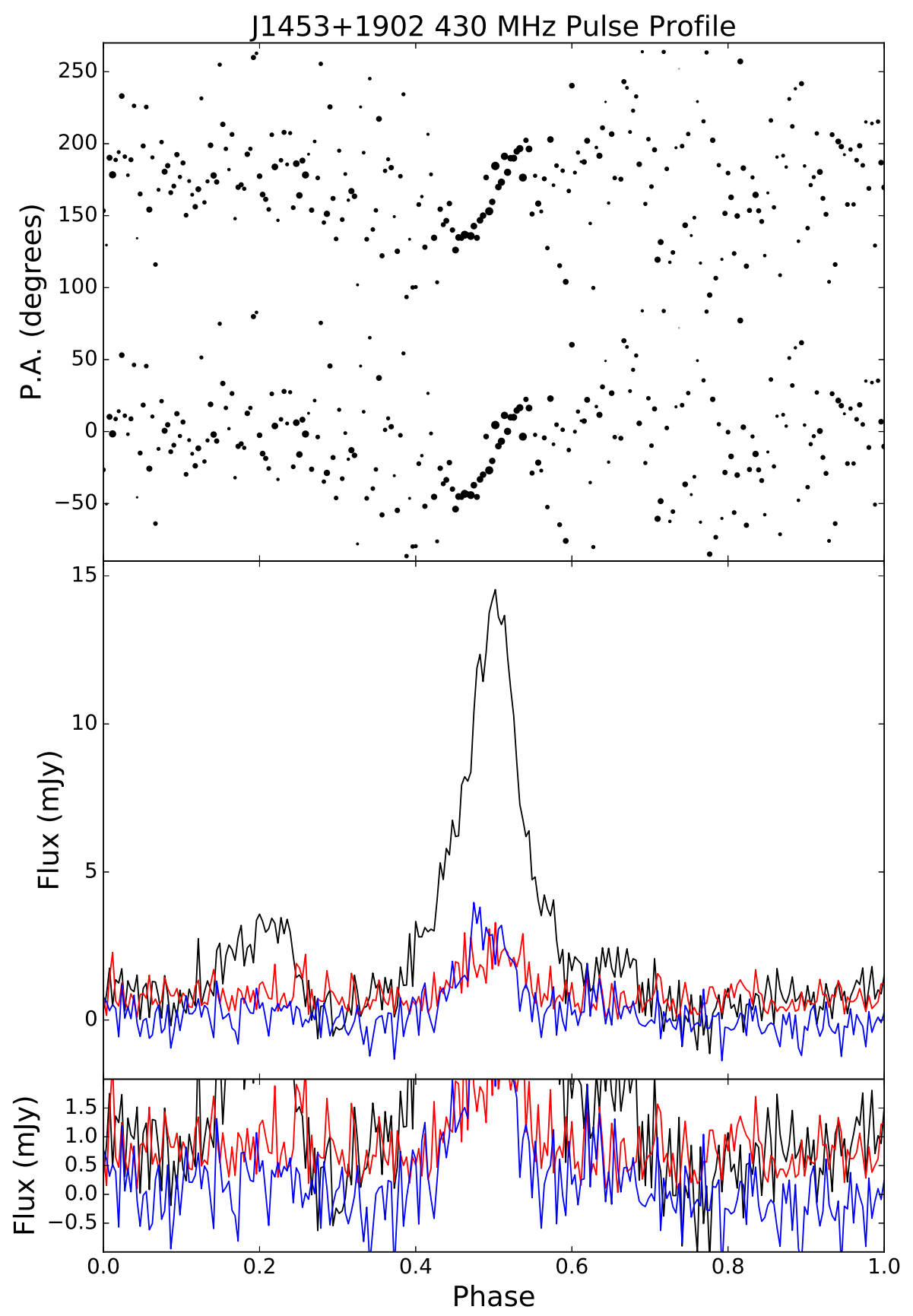

Figure A.8: Same as Figure A.1, for PSR J1453+1902 at $430 \mathrm{MHz}$. 


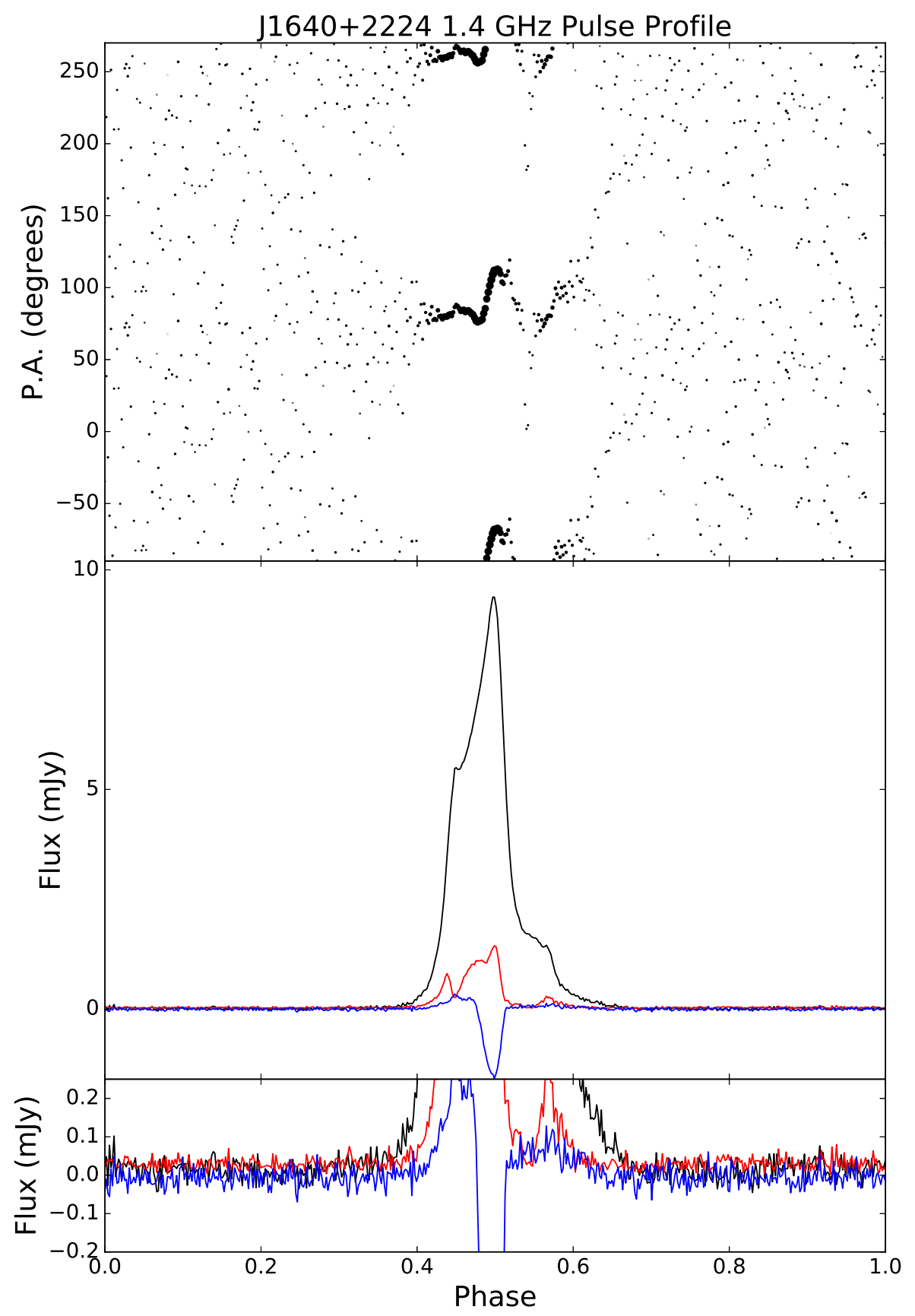

Figure A.9: Same as Figure A.1, for PSR J1640+2224 at 1.4 GHz. 


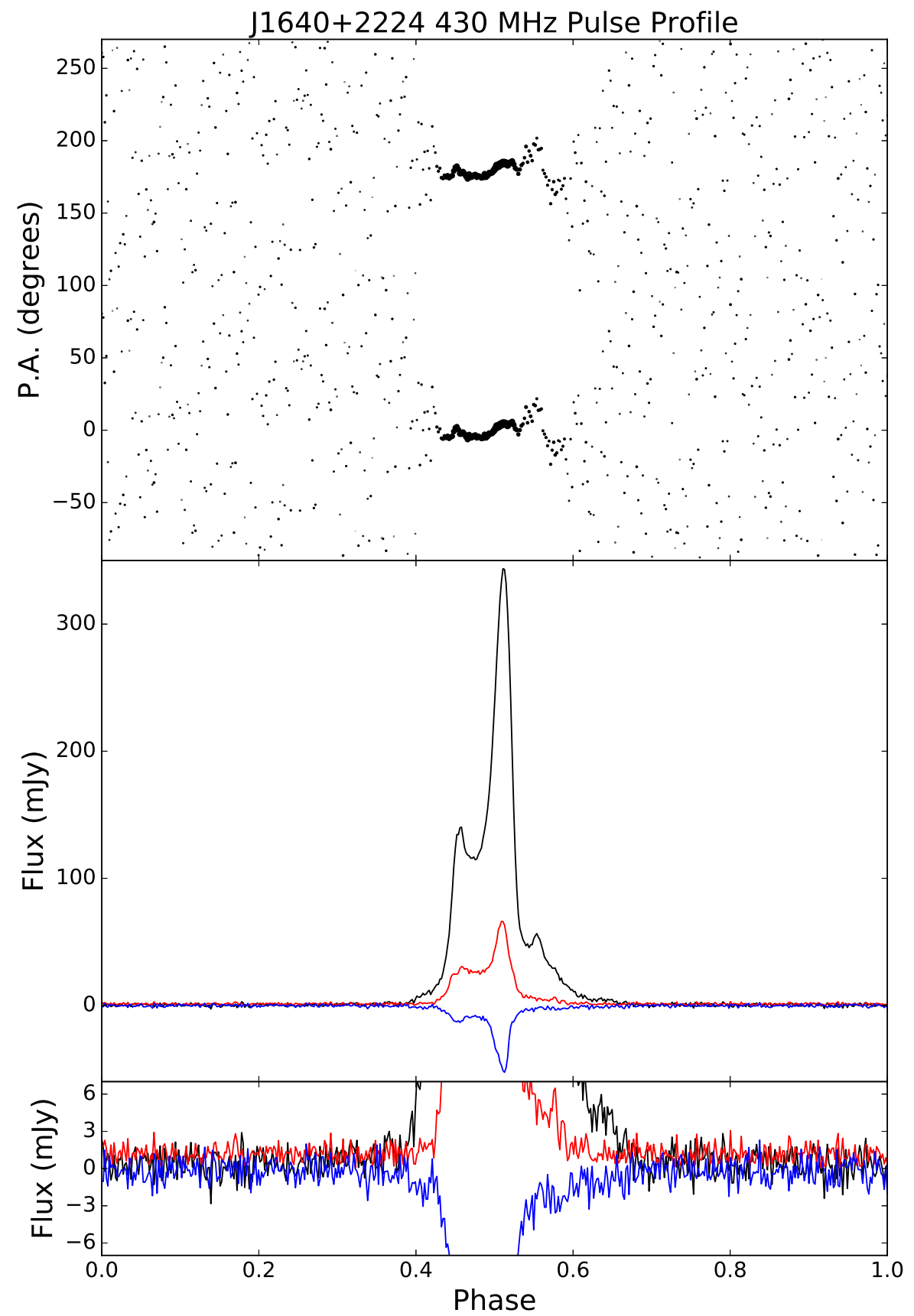

Figure A.10: Same as Figure A.1, for PSR J1640+2224 at 430 MHz. 


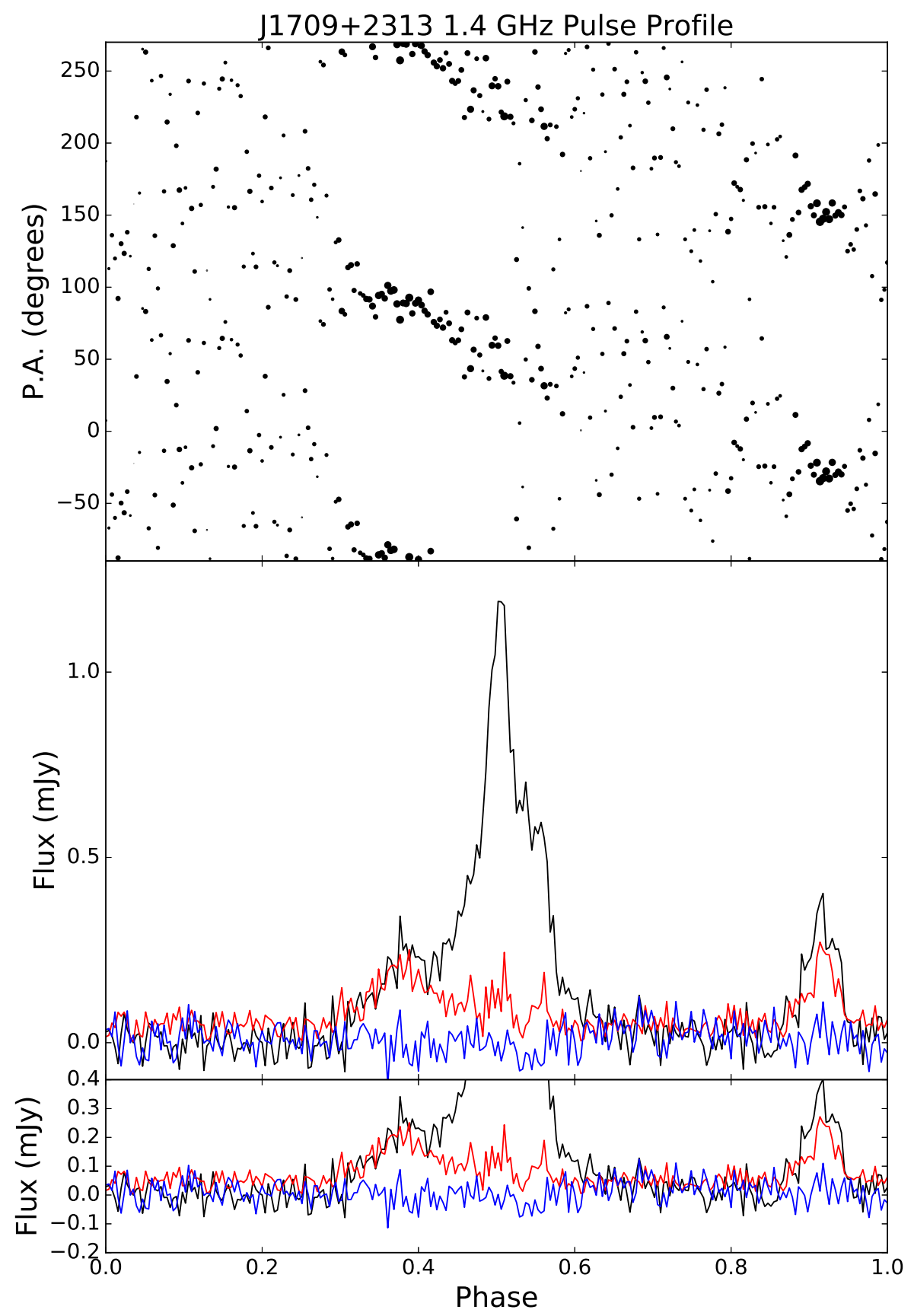

Figure A.11: Same as Figure A.1, for PSR J1709+2313 at 1.4 GHz. 


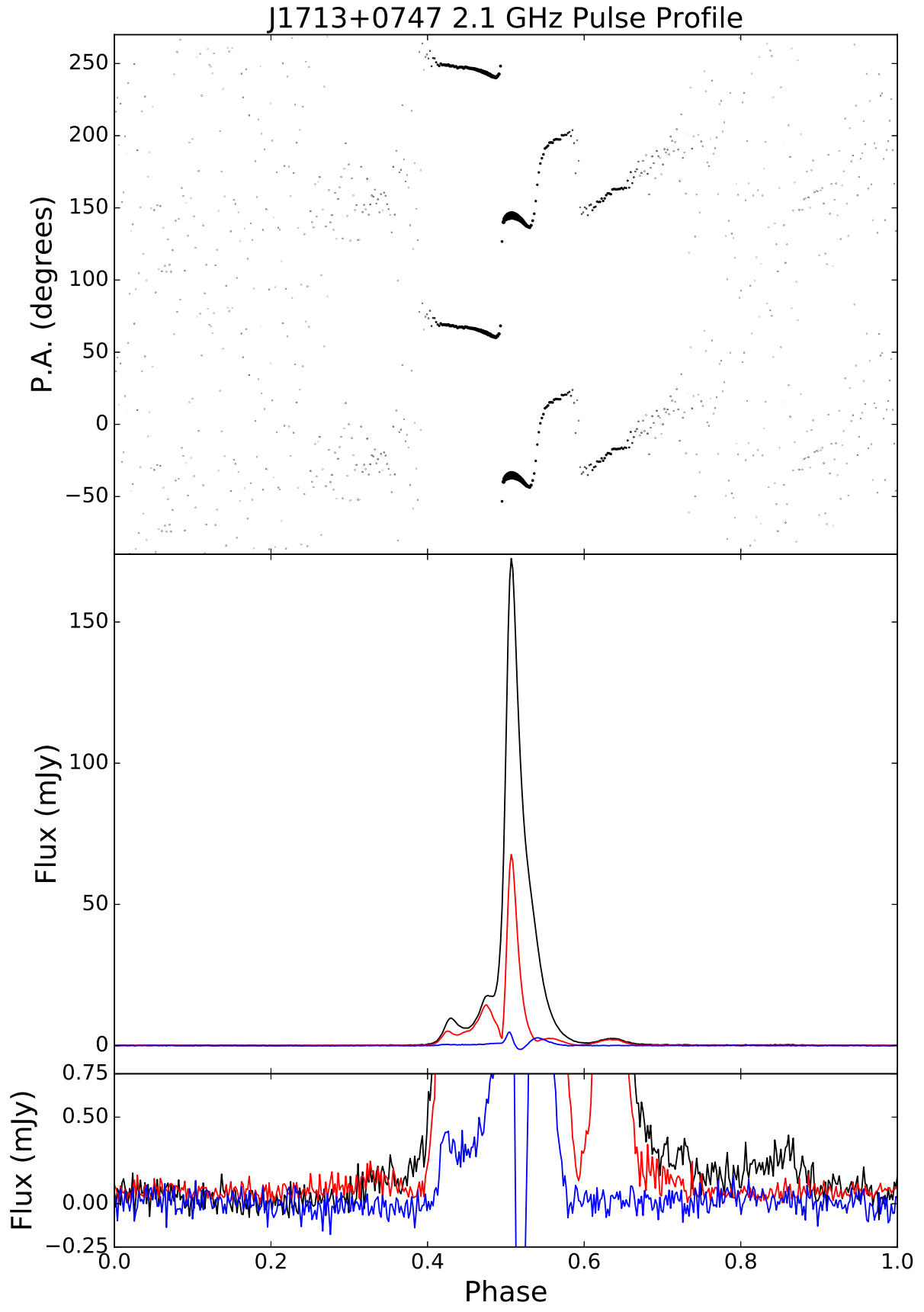

Figure A.12: Same as Figure A.1, for PSR J1713+0747 at 2.1 GHz. 


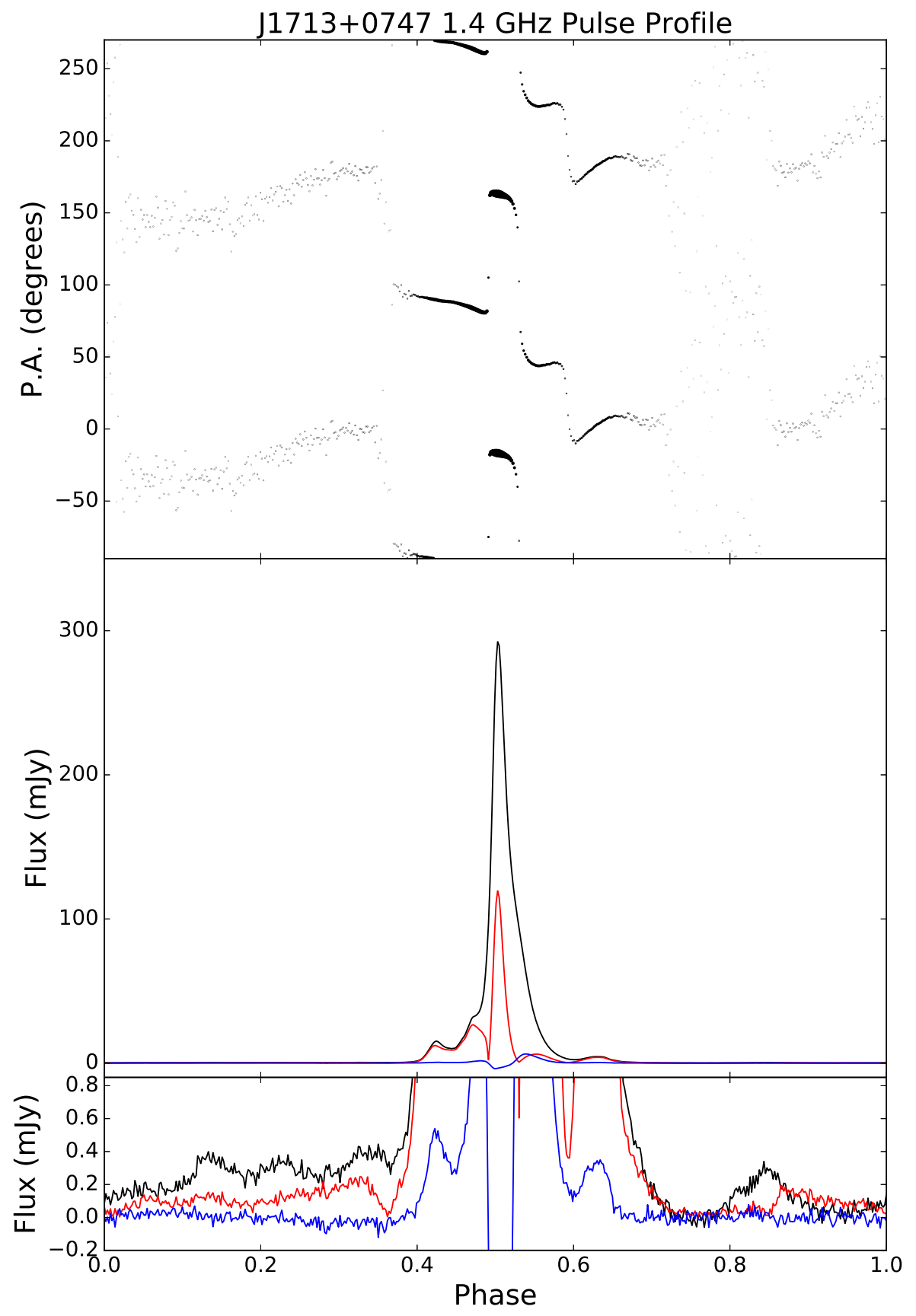

Figure A.13: Same as Figure A.1, for PSR J1713+0747 at 1.4 GHz. 


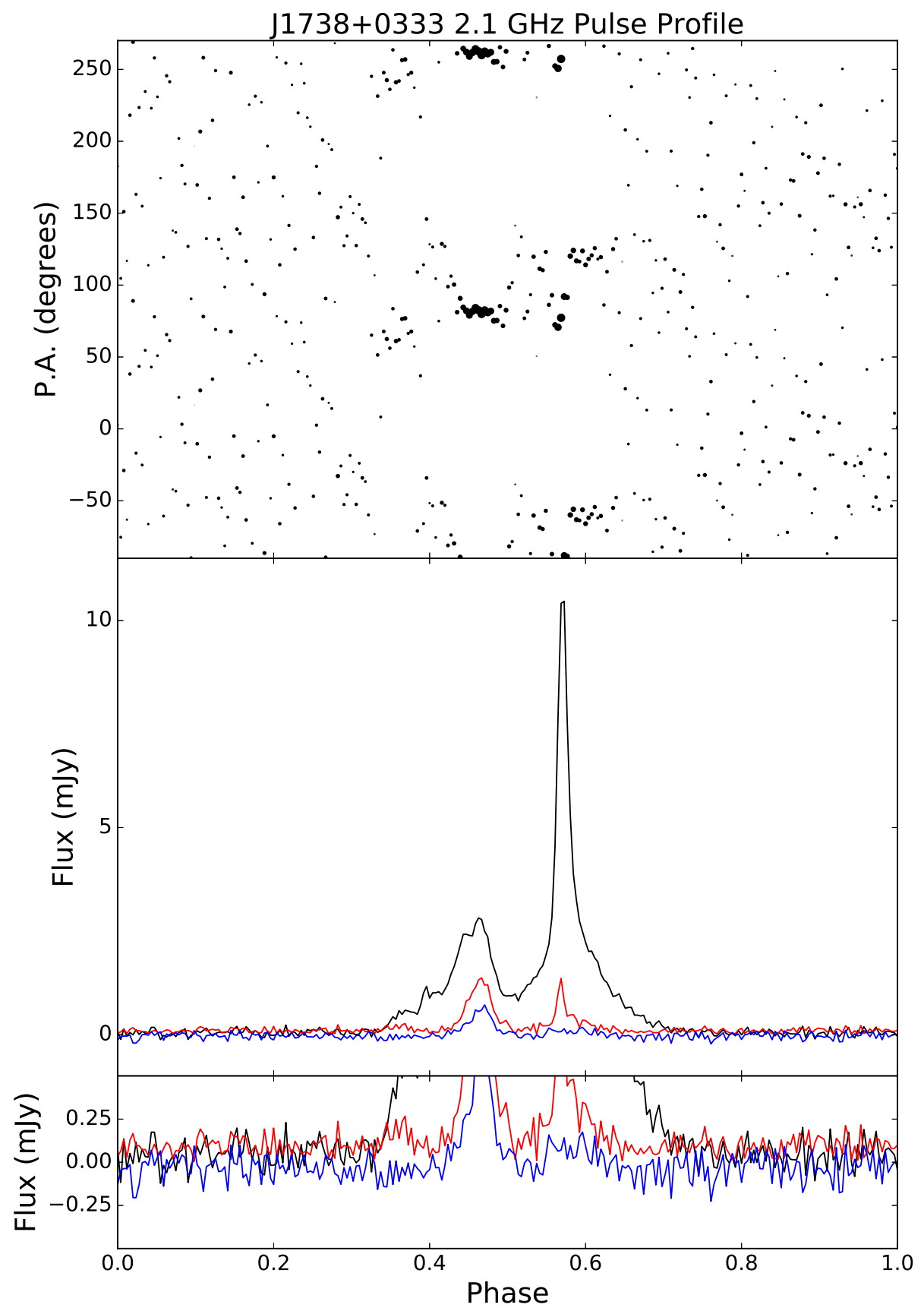

Figure A.14: Same as Figure A.1, for PSR J1738+0333 at 2.1 GHz. 


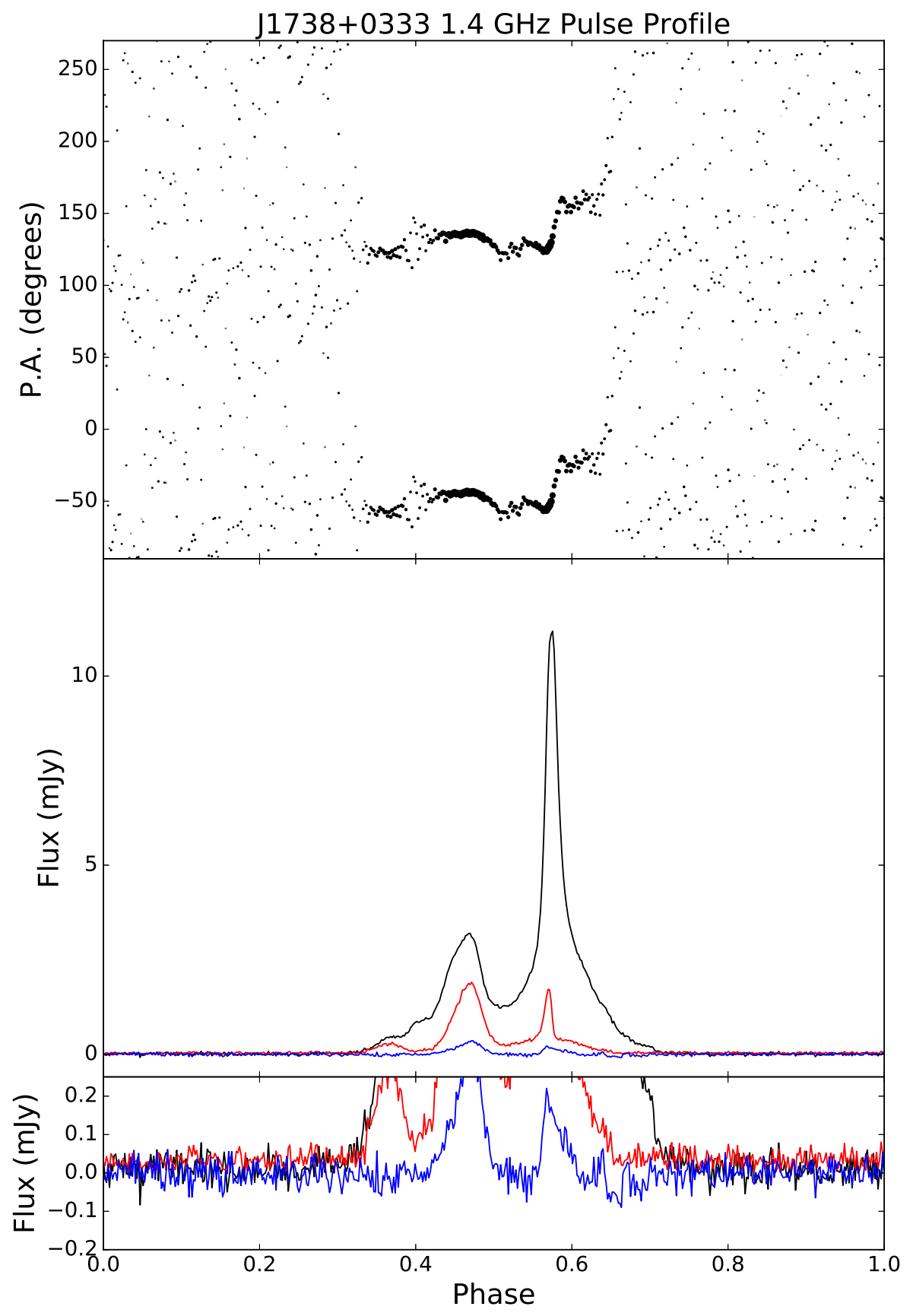

Figure A.15: Same as Figure A.1, for PSR J1738+0333 at 1.4 GHz. 


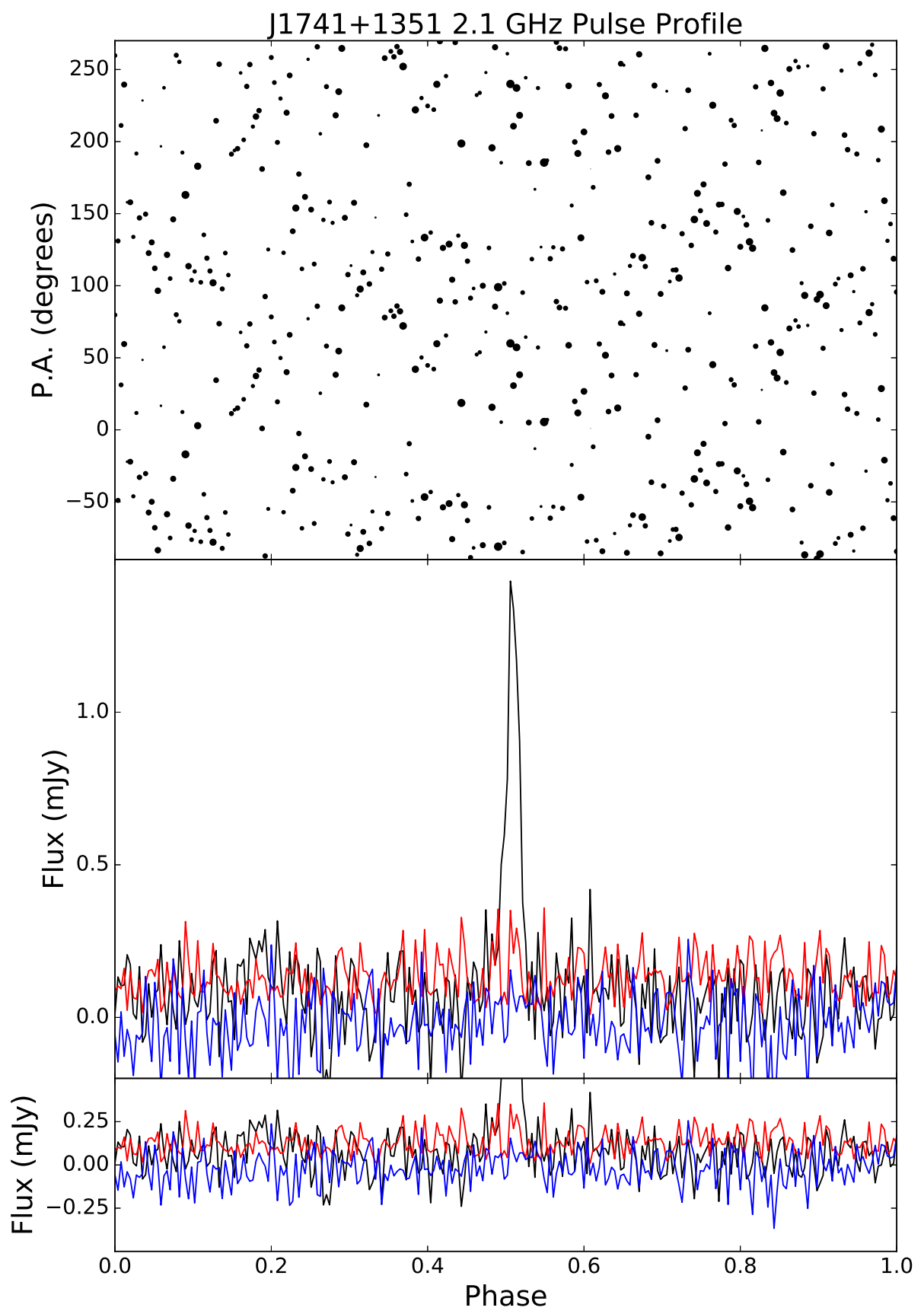

Figure A.16: Same as Figure A.1, for PSR J1741+1351 at $2.1 \mathrm{GHz}$. 


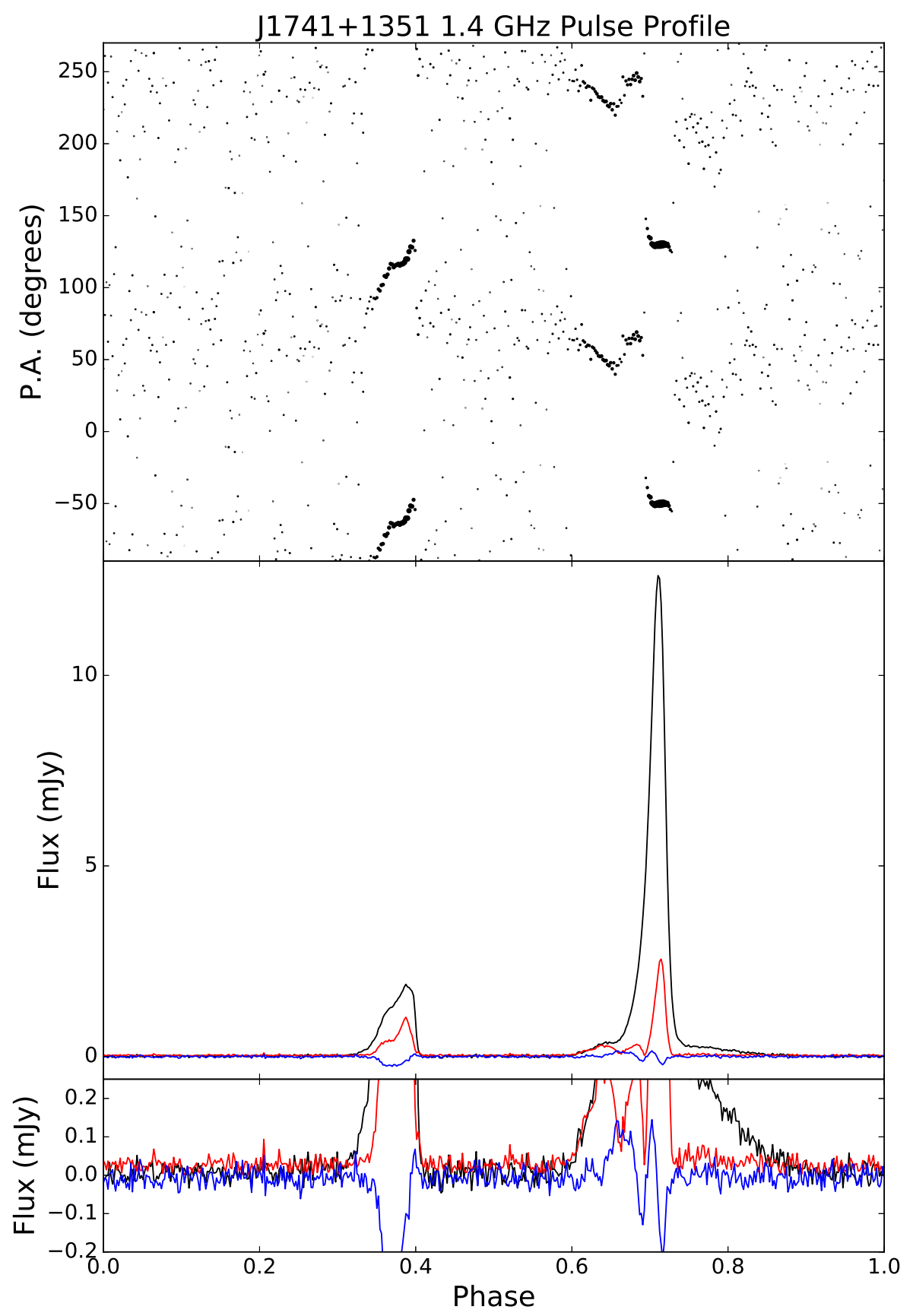

Figure A.17: Same as Figure A.1, for PSR J1741+1351 at 1.4 GHz. 


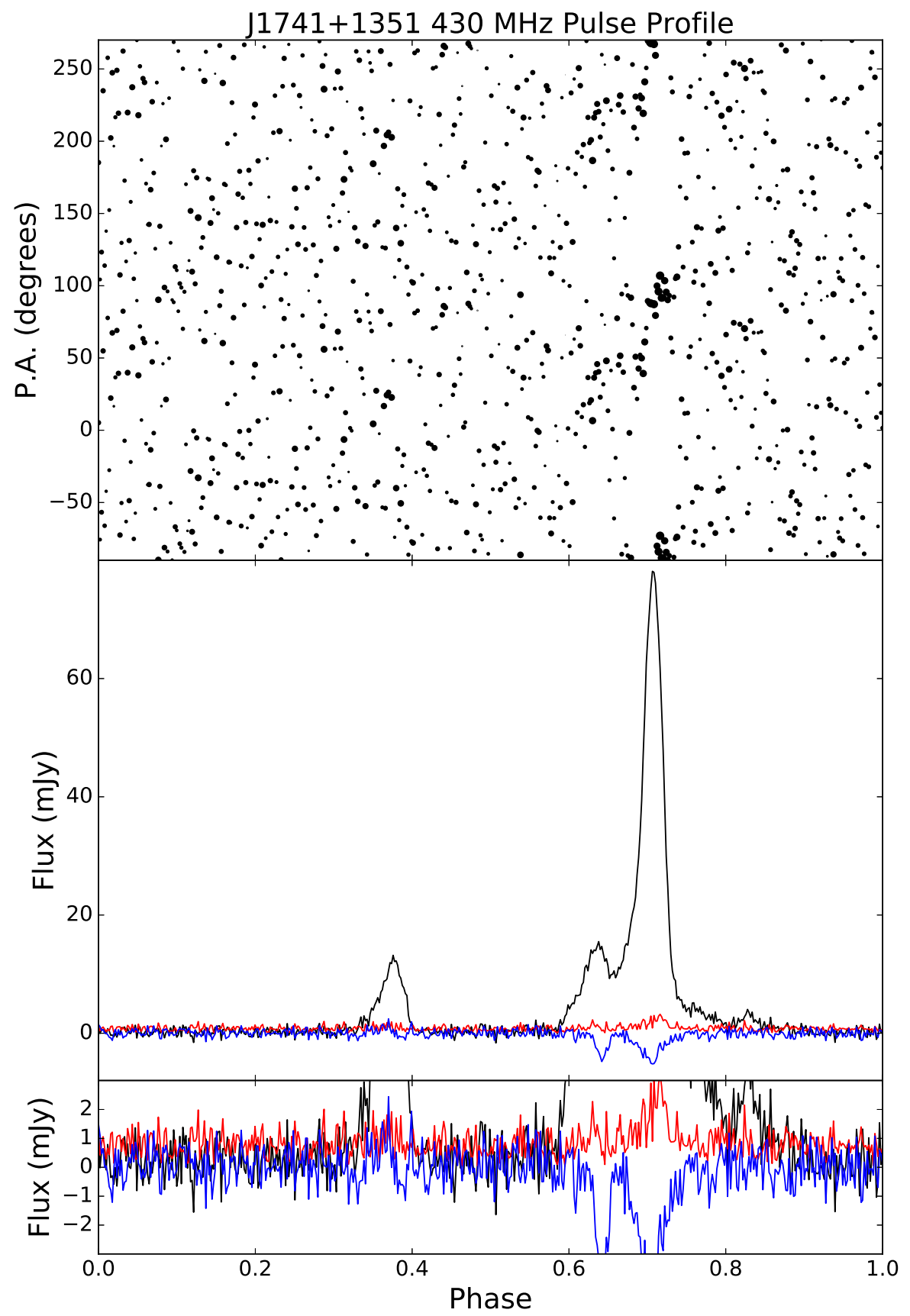

Figure A.18: Same as Figure A.1, for PSR J1741+1351 at 430 MHz. 


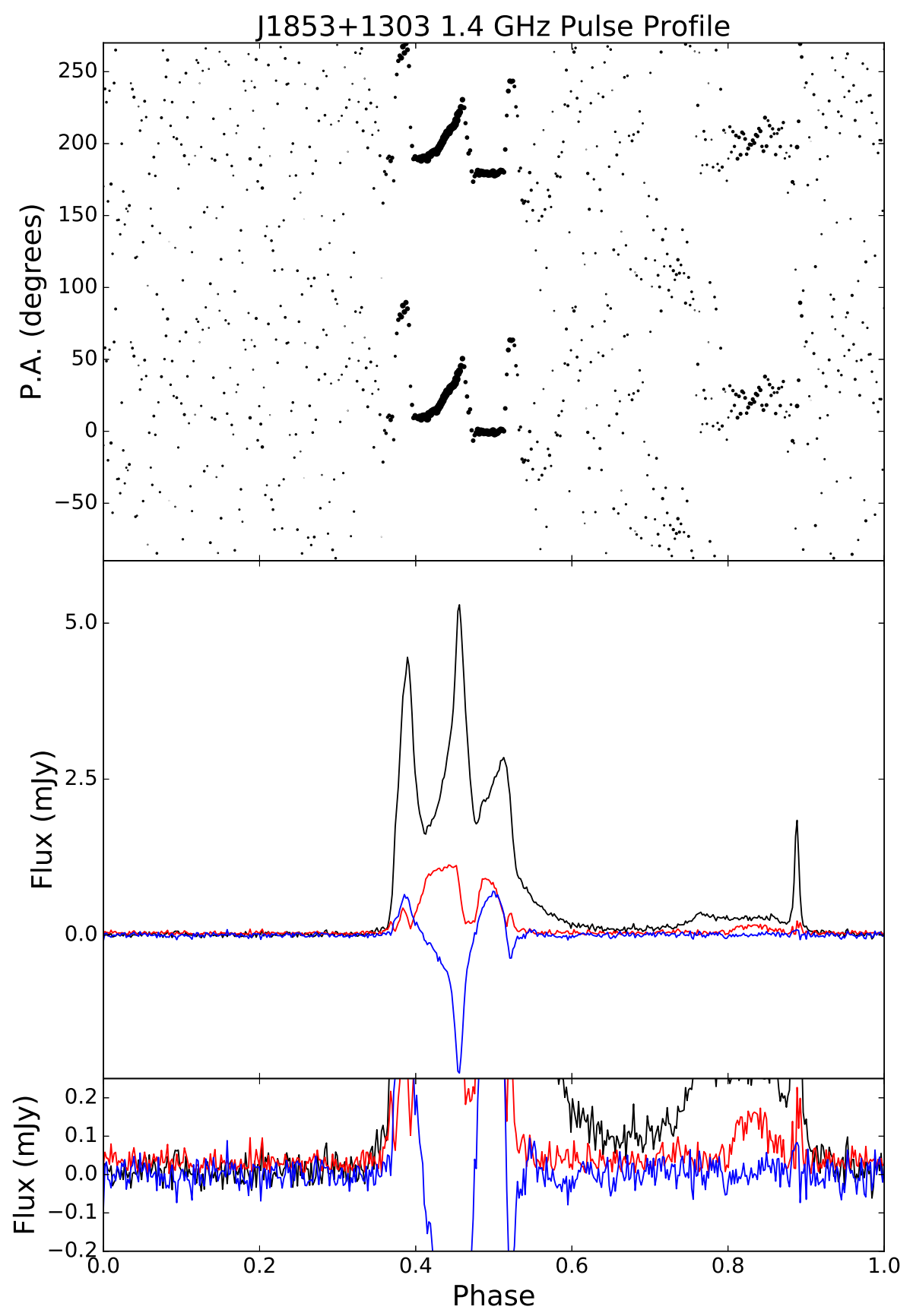

Figure A.19: Same as Figure A.1, for PSR J1853+1303 at 1.4 GHz. 


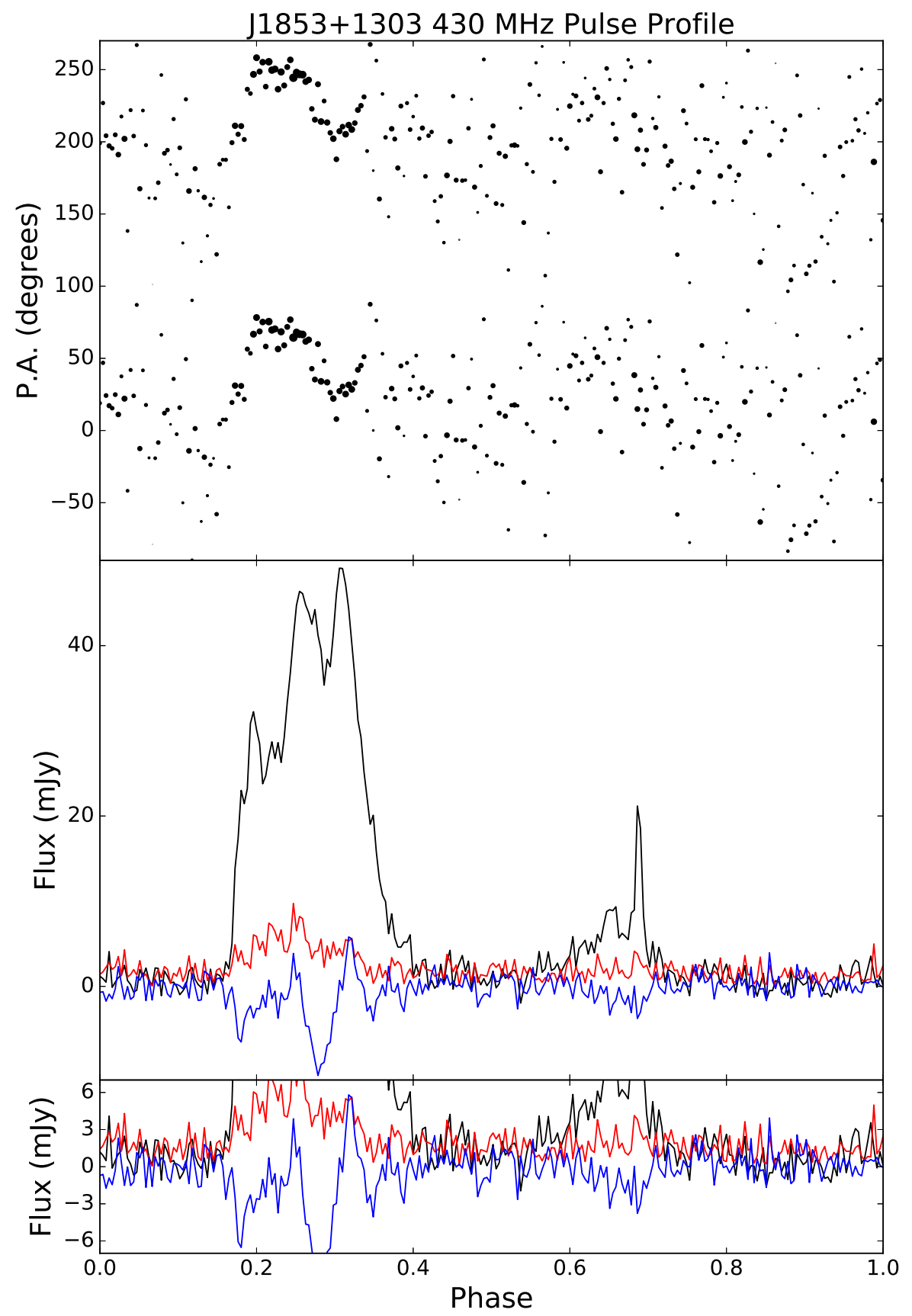

Figure A.20: Same as Figure A.1, for PSR J1853+1303 at 430 MHz. 


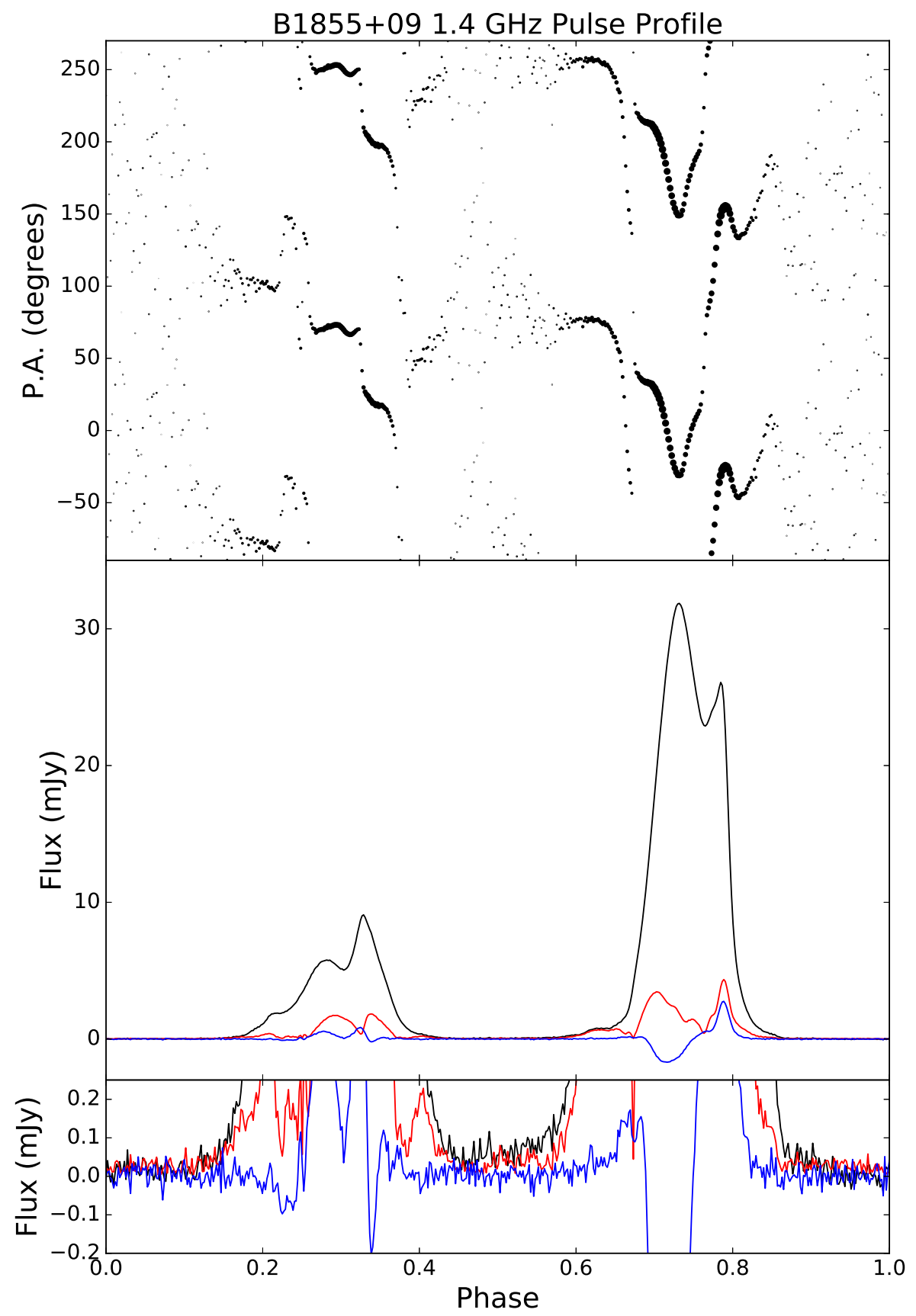

Figure A.21: Same as Figure A.1, for PSR B1855+09 at 1.4 GHz. 


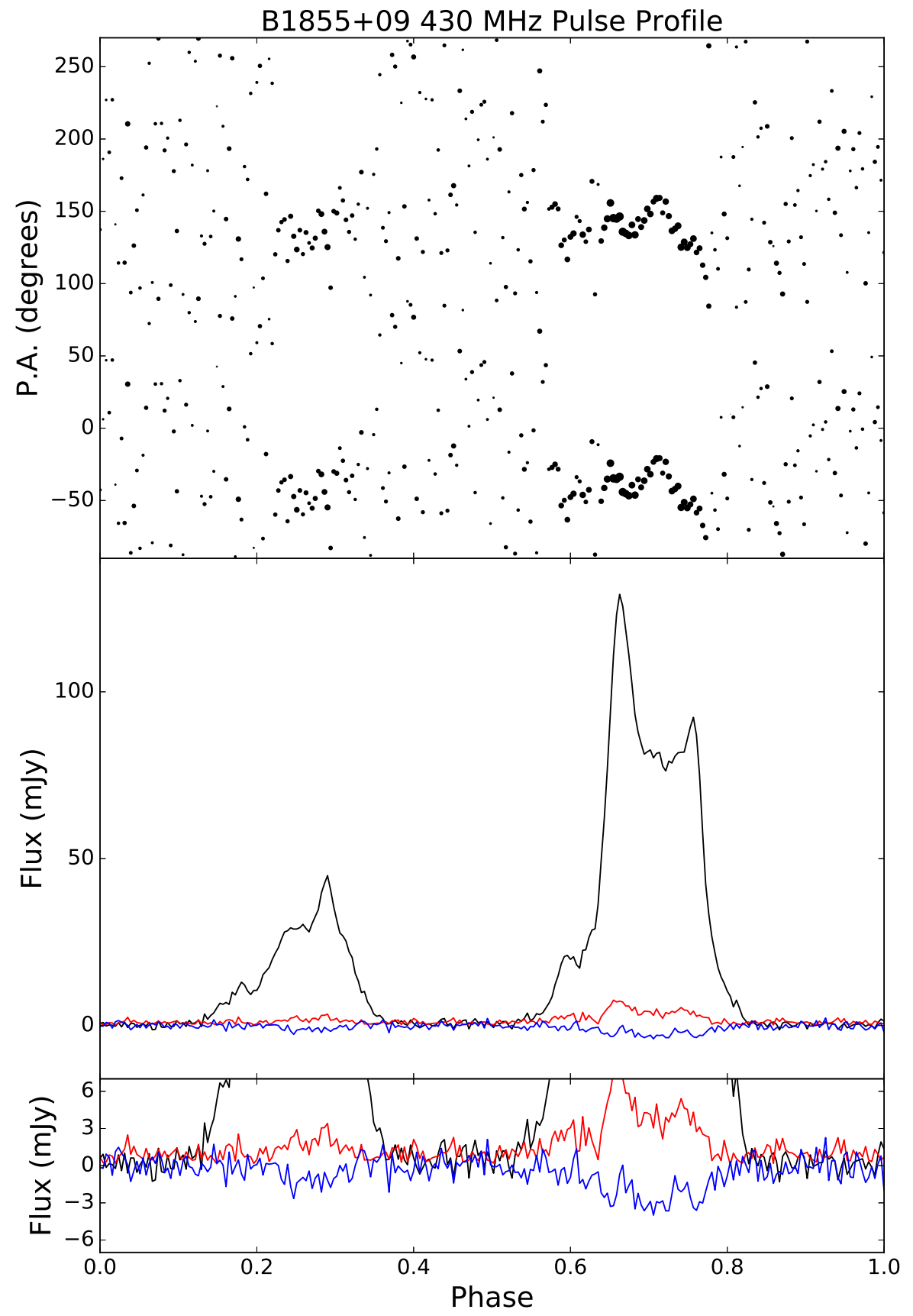

Figure A.22: Same as Figure A.1, for PSR B1855+09 at 430 MHz. 


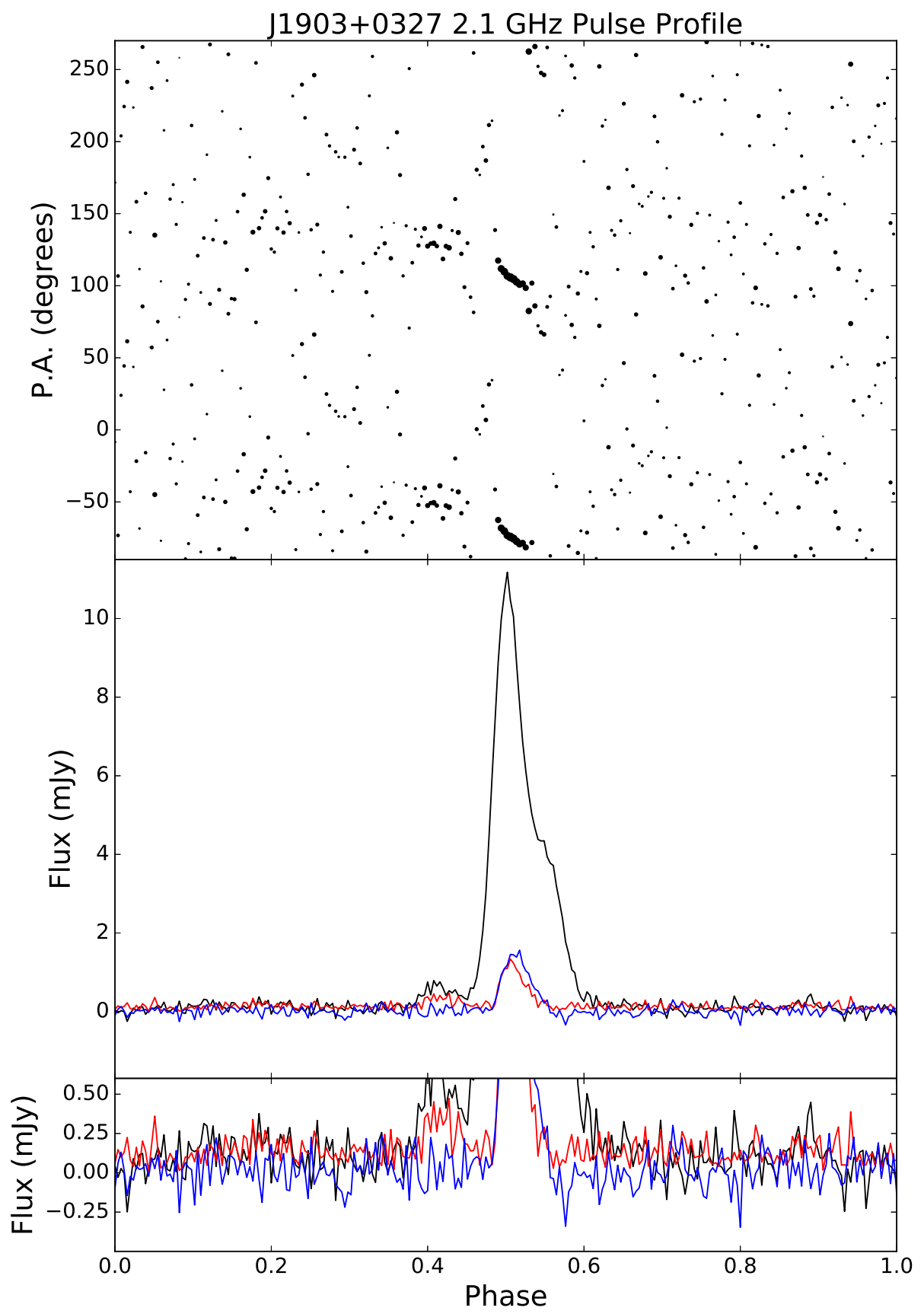

Figure A.23: Same as Figure A.1, for PSR J1903+0327 at 2.1 GHz. 


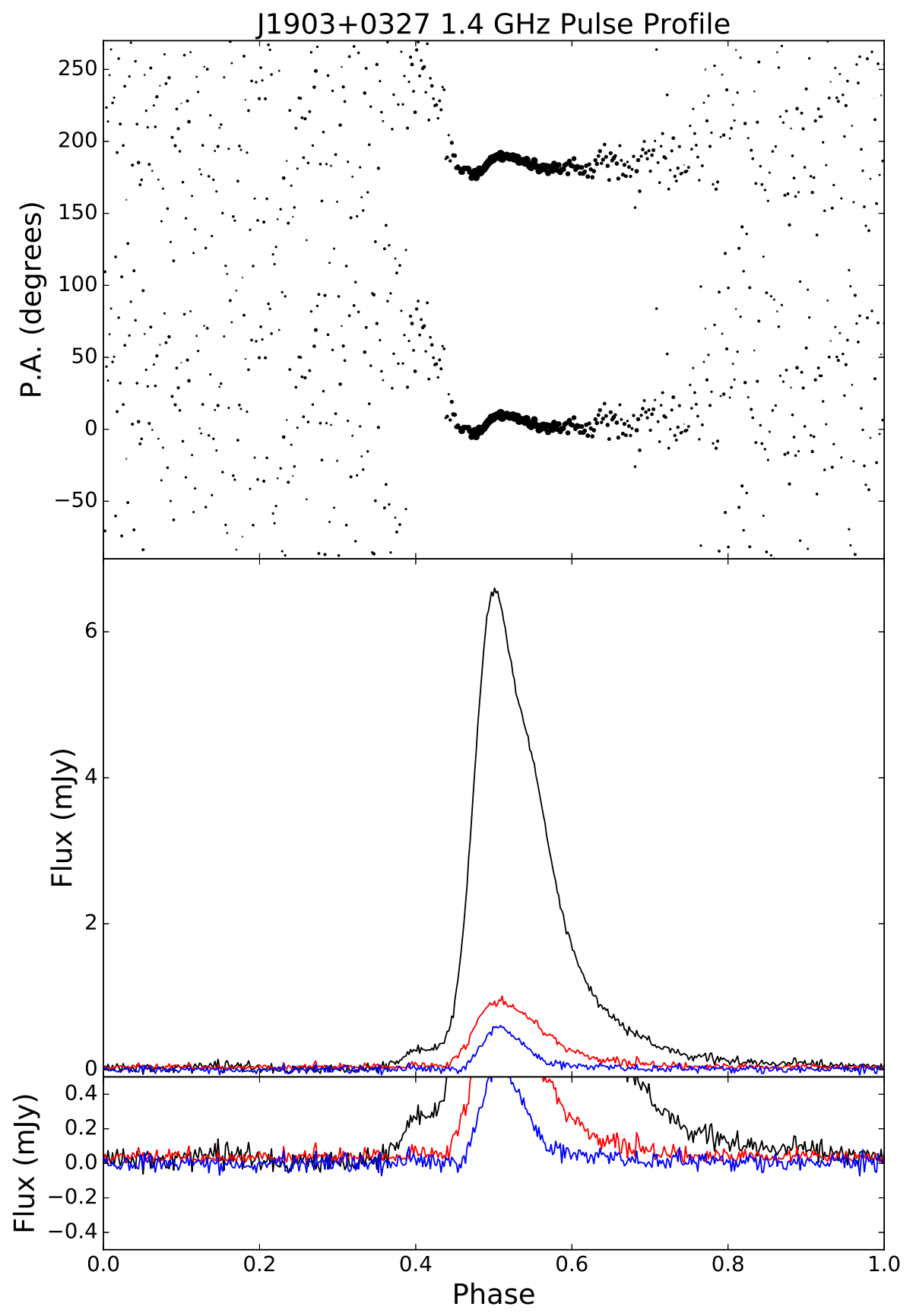

Figure A.24: Same as Figure A.1, for PSR J1903+0327 at 1.4 GHz. 


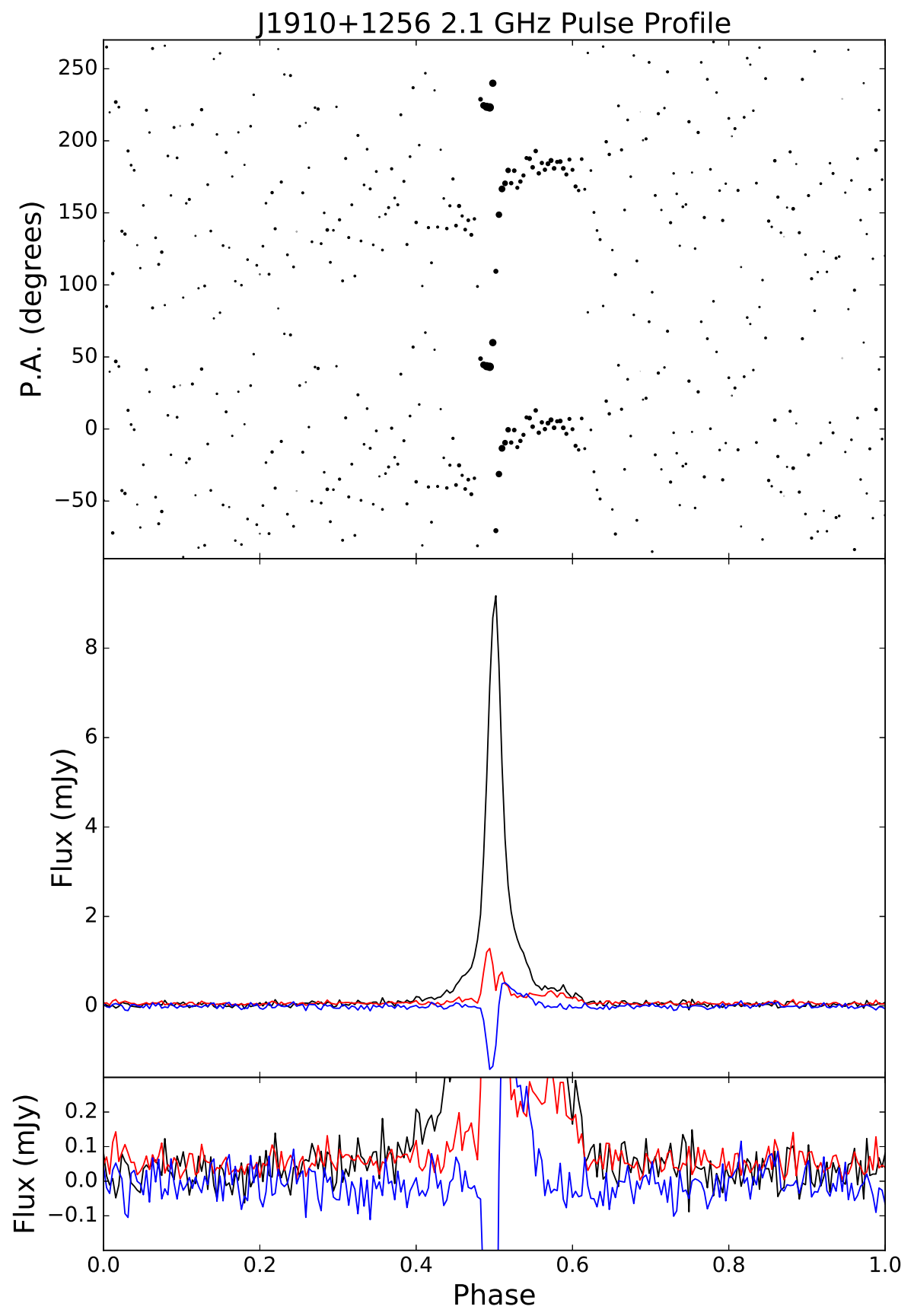

Figure A.25: Same as Figure A.1, for PSR J1910+1256 at 2.1 GHz. 


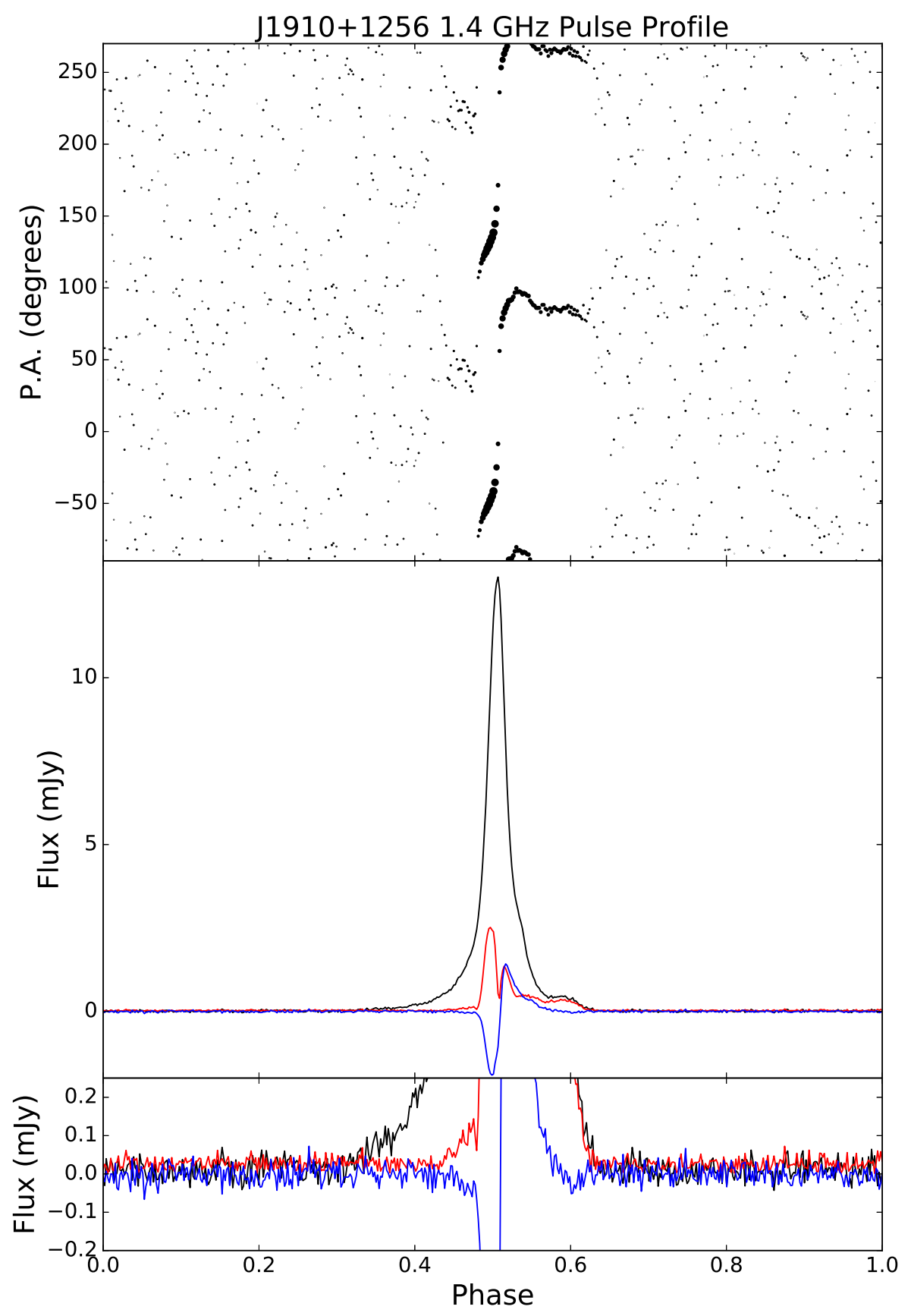

Figure A.26: Same as Figure A.1, for PSR J1910+1256 at 1.4 GHz. 


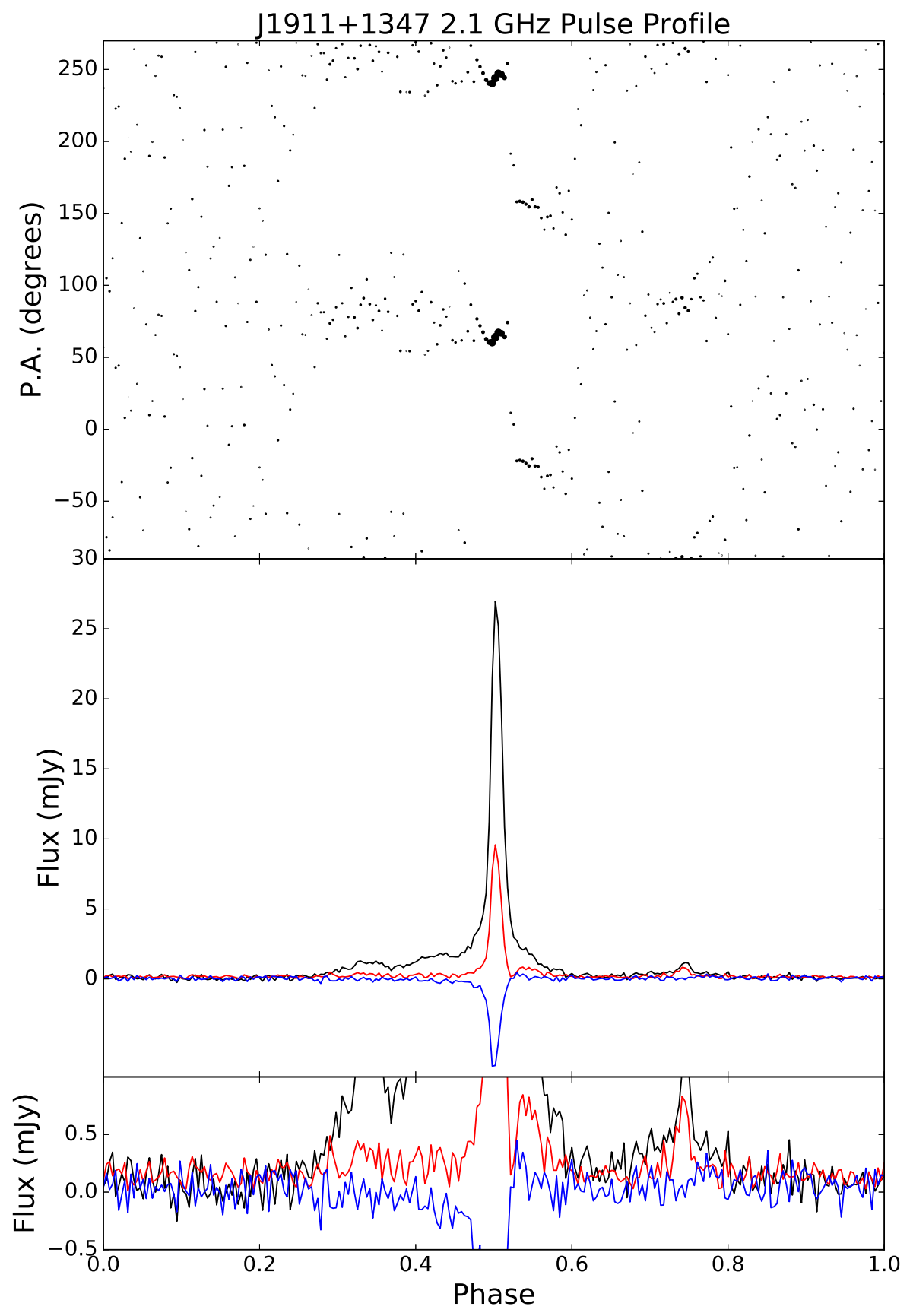

Figure A.27: Same as Figure A.1, for PSR J1911+1347 at 2.1 GHz. 


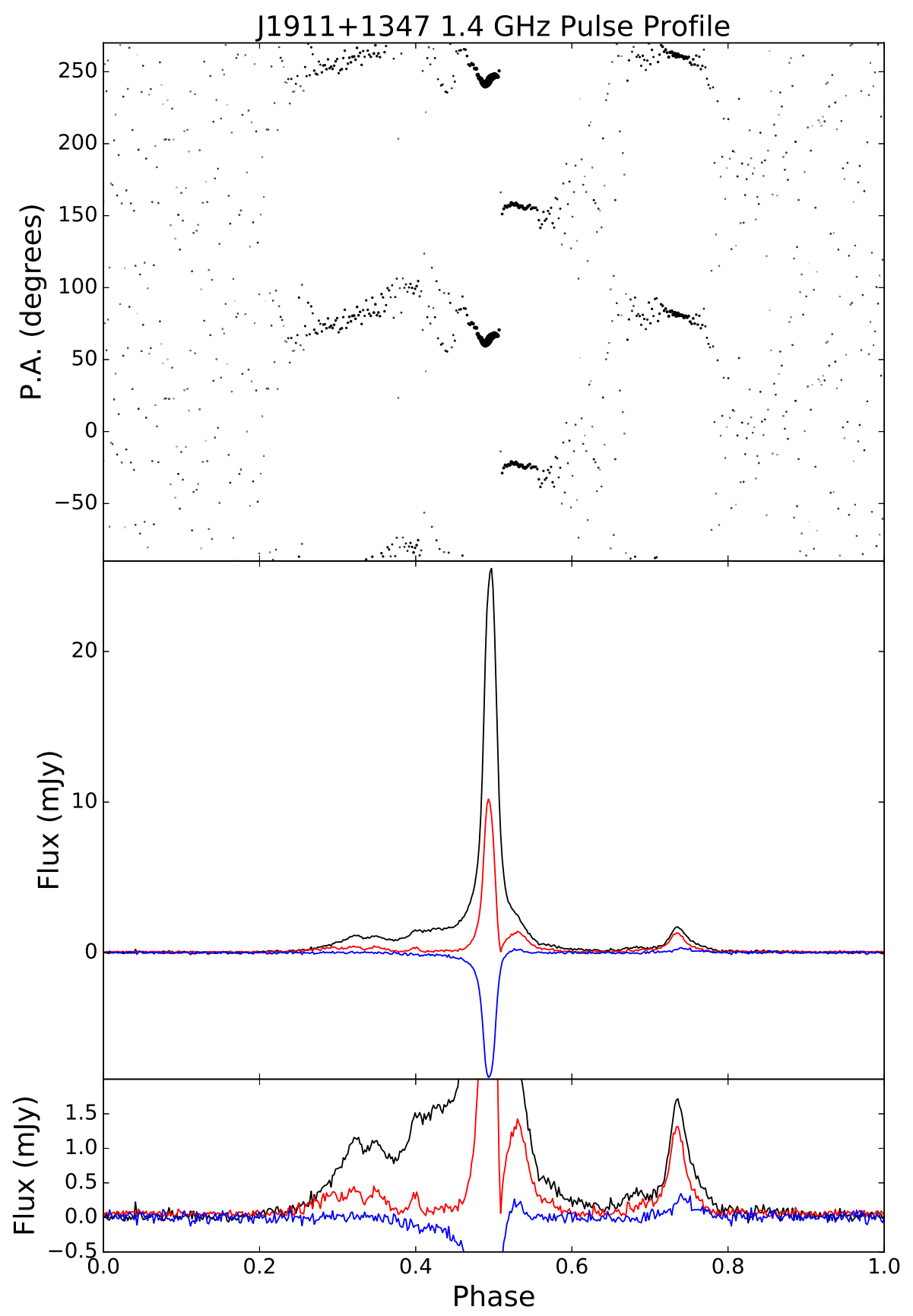

Figure A.28: Same as Figure A.1, for PSR J1911+1347 at 1.4 GHz. 


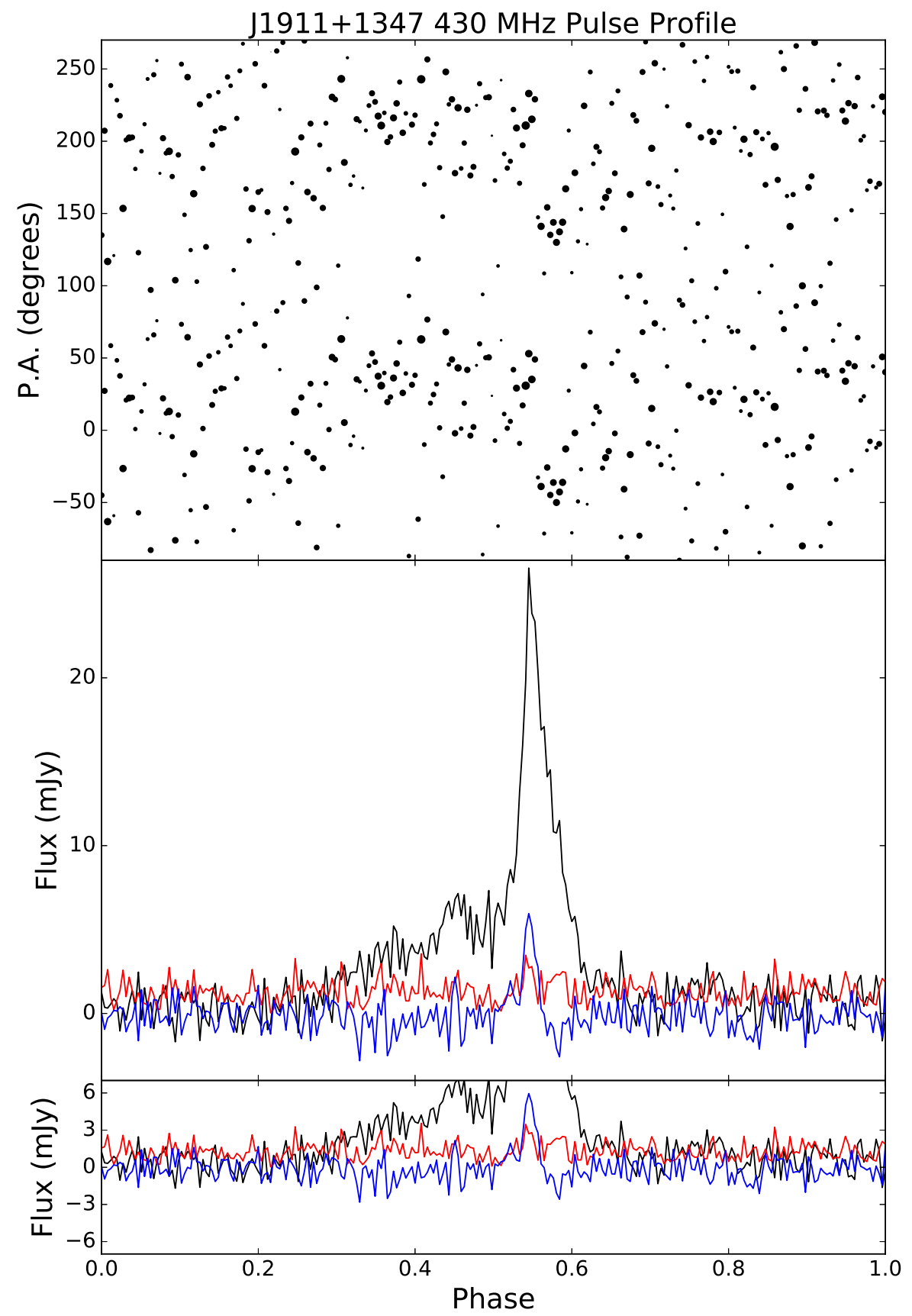

Figure A.29: Same as Figure A.1, for PSR J1911+1347 at 430 MHz. 


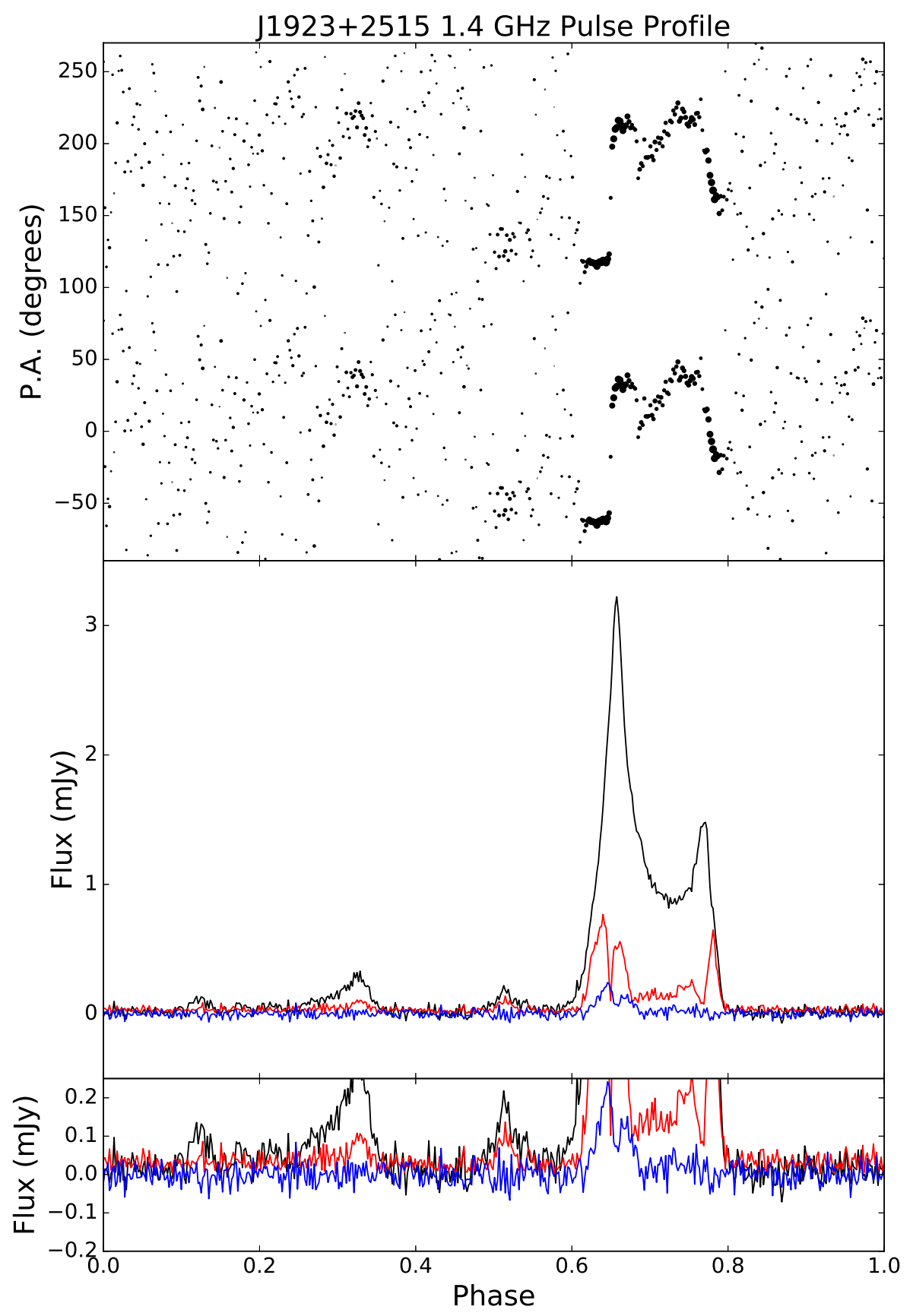

Figure A.30: Same as Figure A.1, for PSR J1923+2515 at 1.4 GHz. 


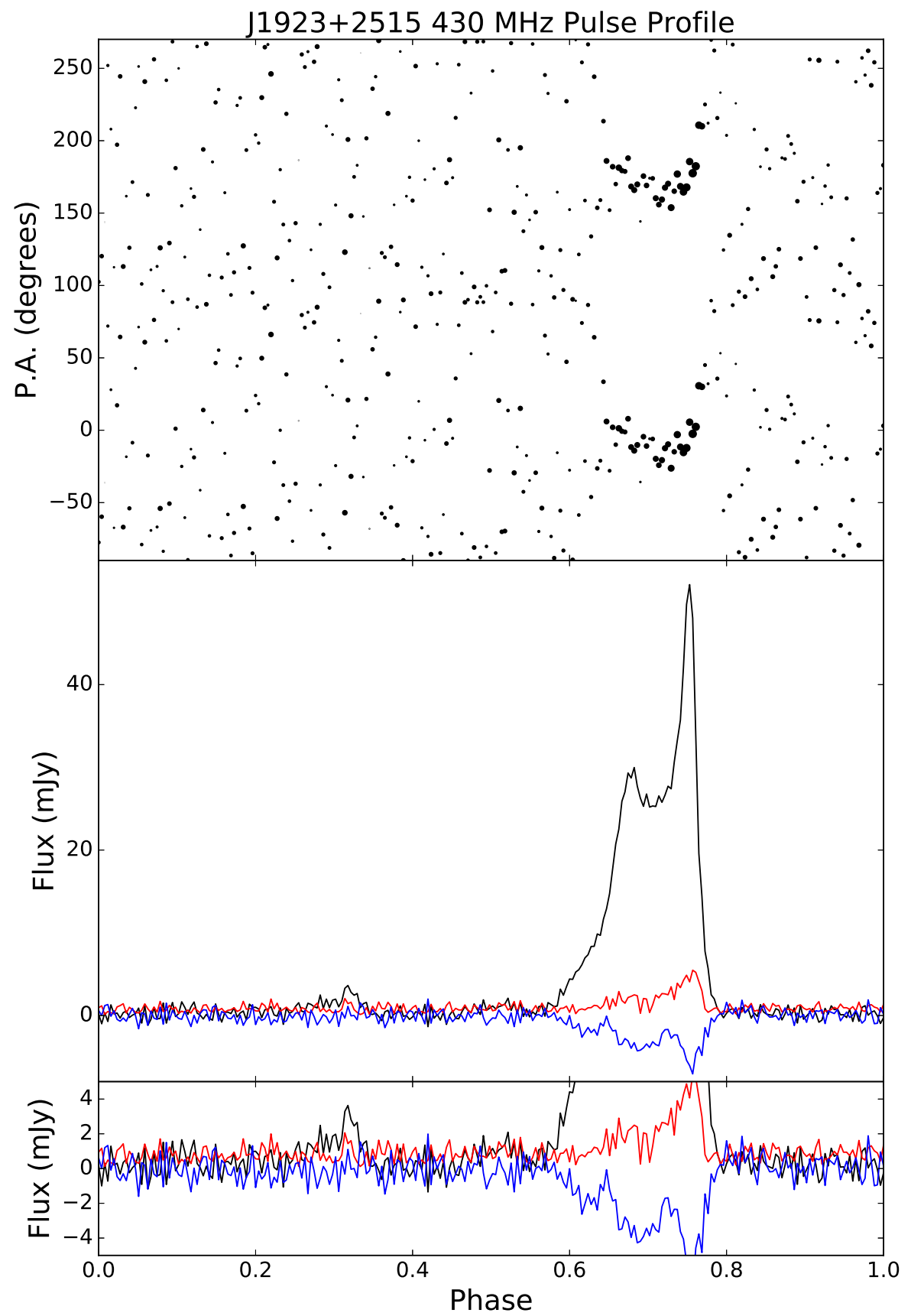

Figure A.31: Same as Figure A.1, for PSR J1923+2515 at 430 MHz. 


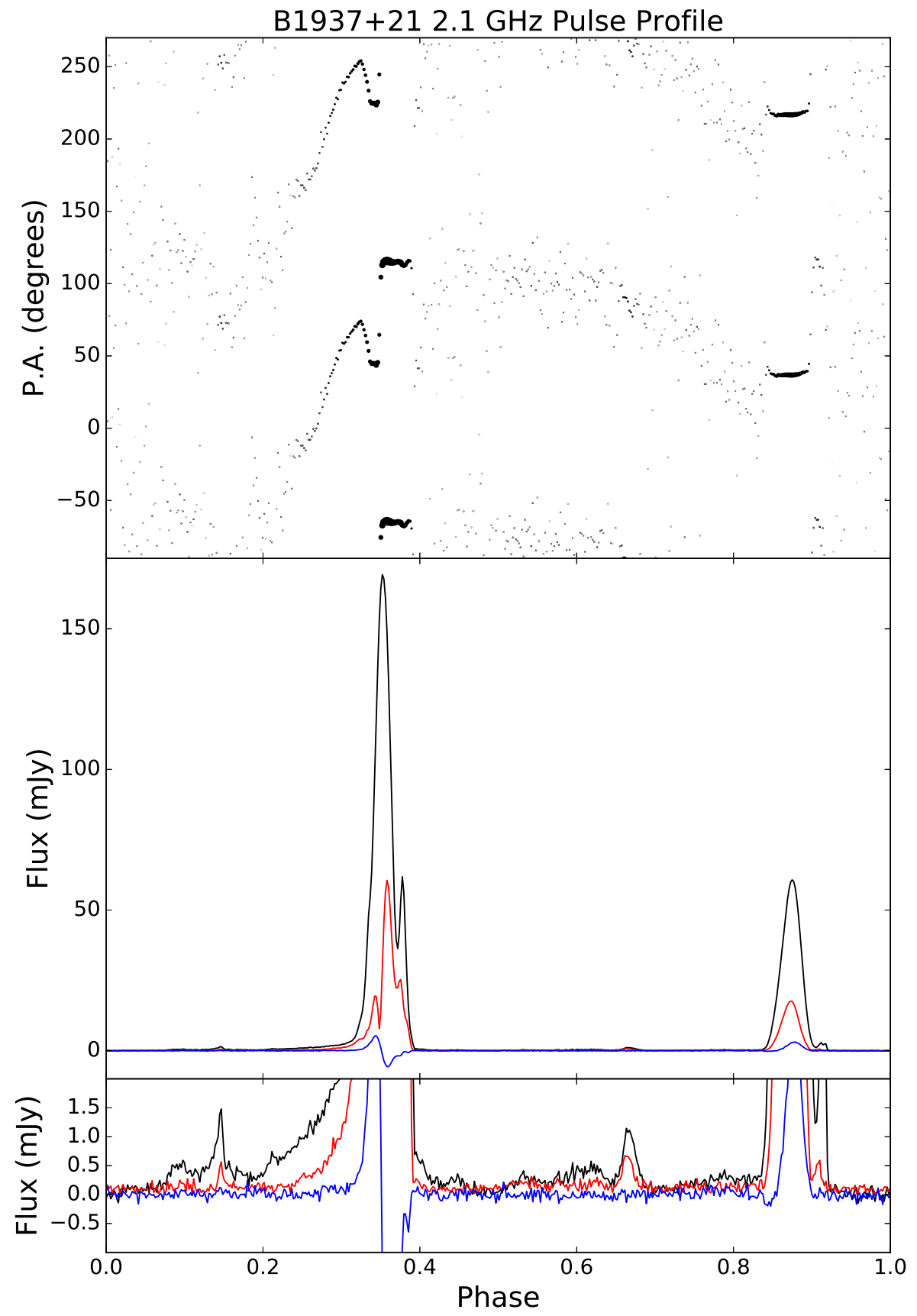

Figure A.32: Same as Figure A.1, for PSR B1937+21 at 2.1 GHz. 


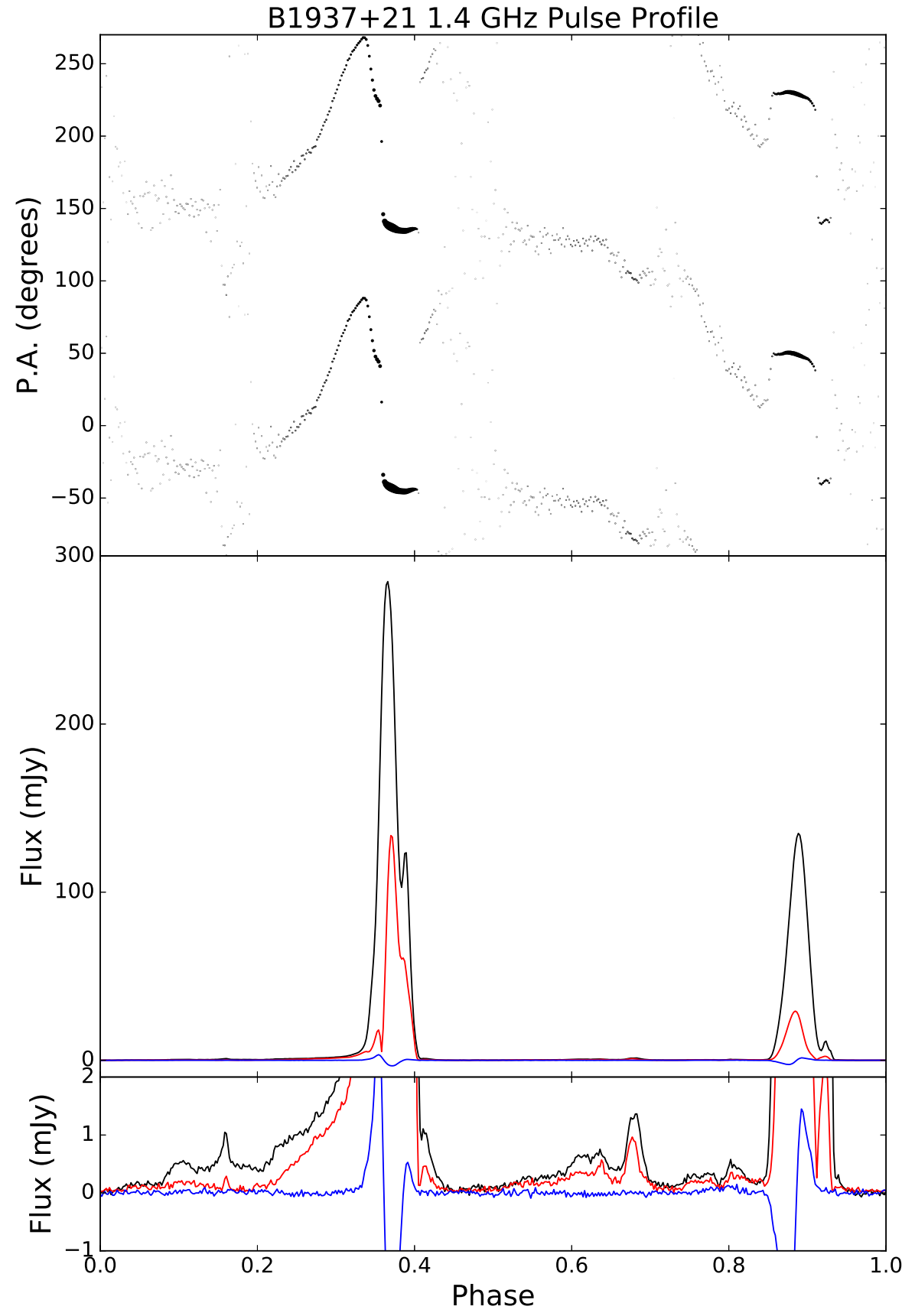

Figure A.33: Same as Figure A.1, for PSR B1937+21 at 1.4 GHz. 


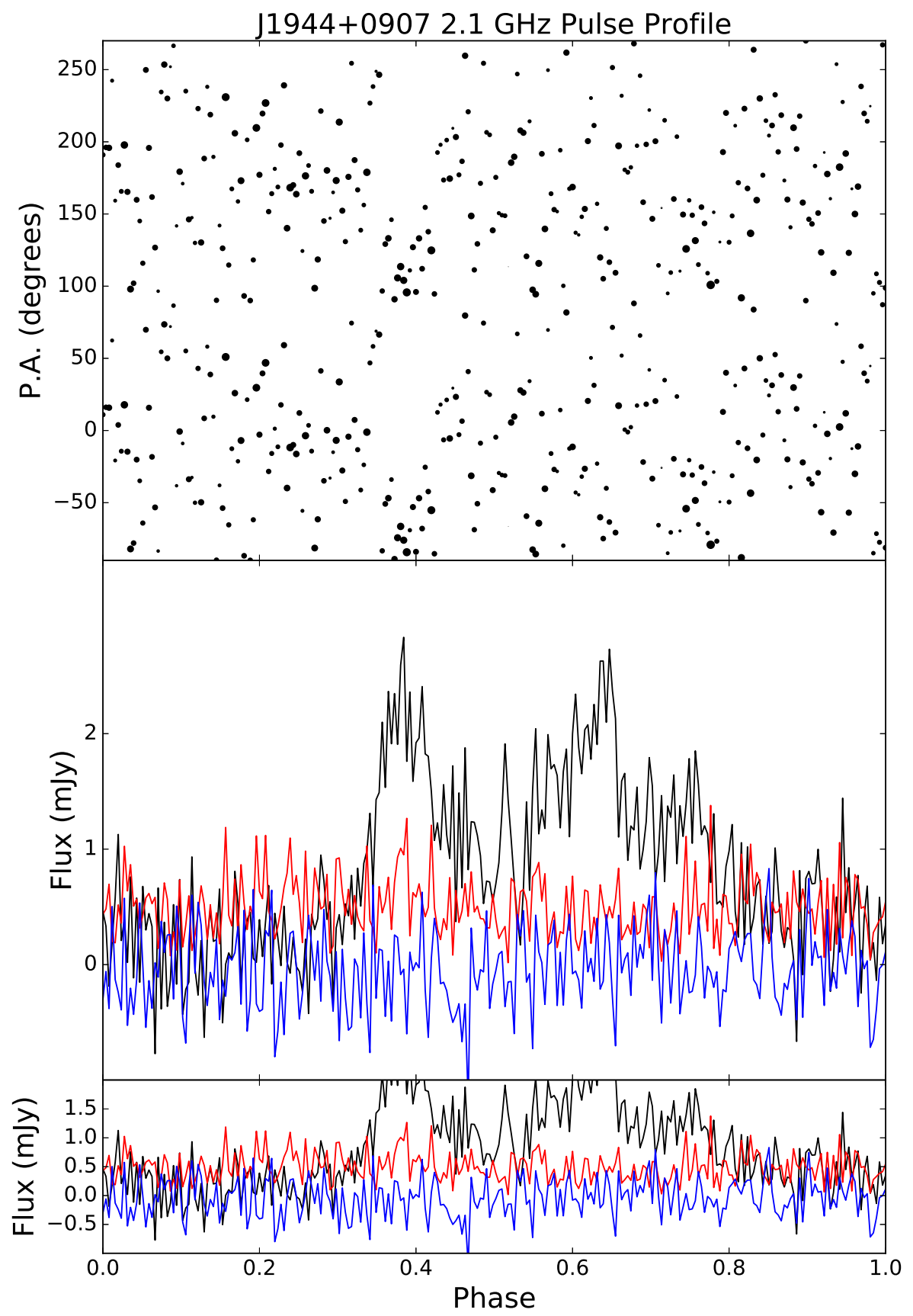

Figure A.34: Same as Figure A.1, for PSR J1944+0907 at 2.1 GHz. 


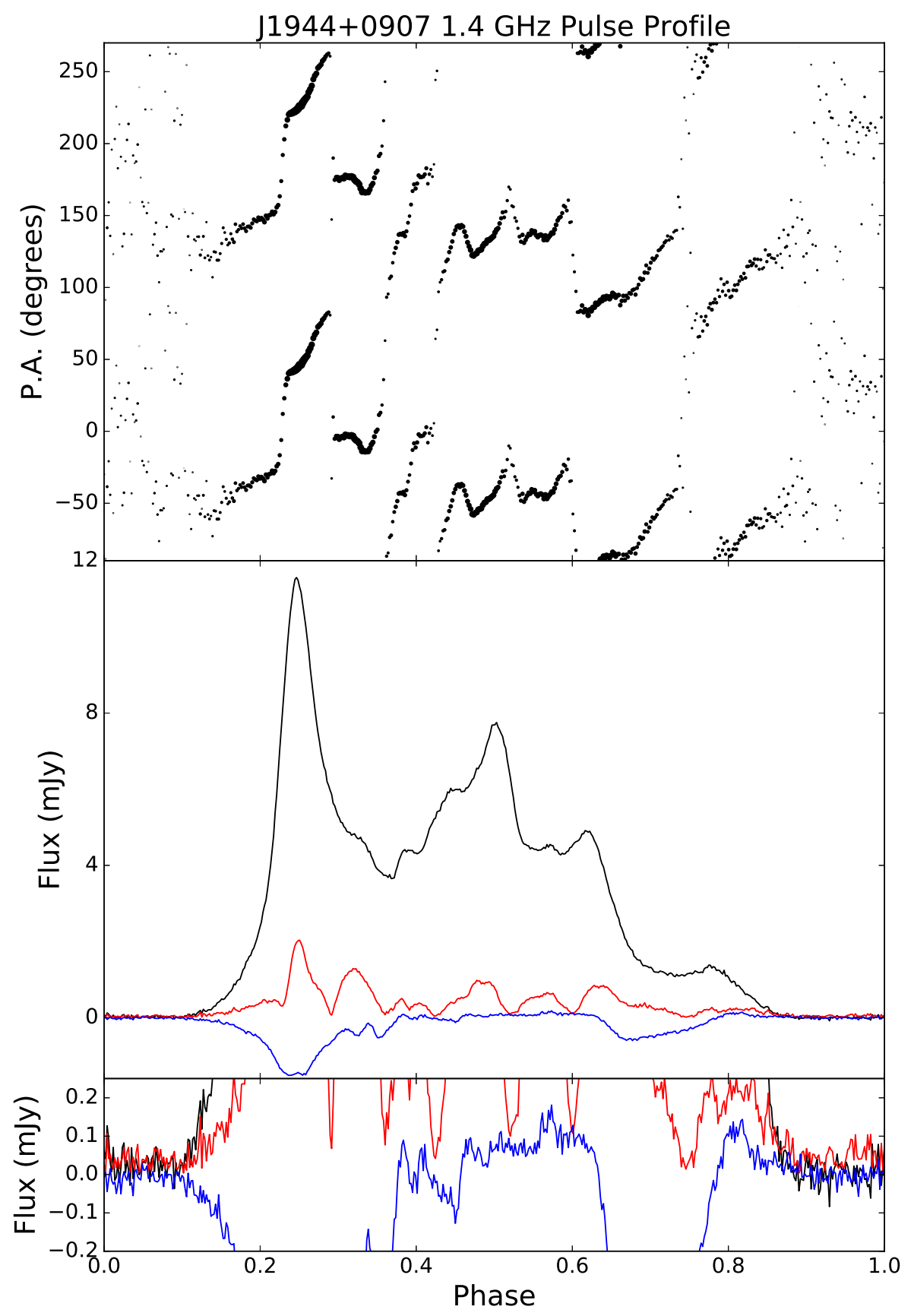

Figure A.35: Same as Figure A.1, for PSR J1944+0907 at 1.4 GHz. 


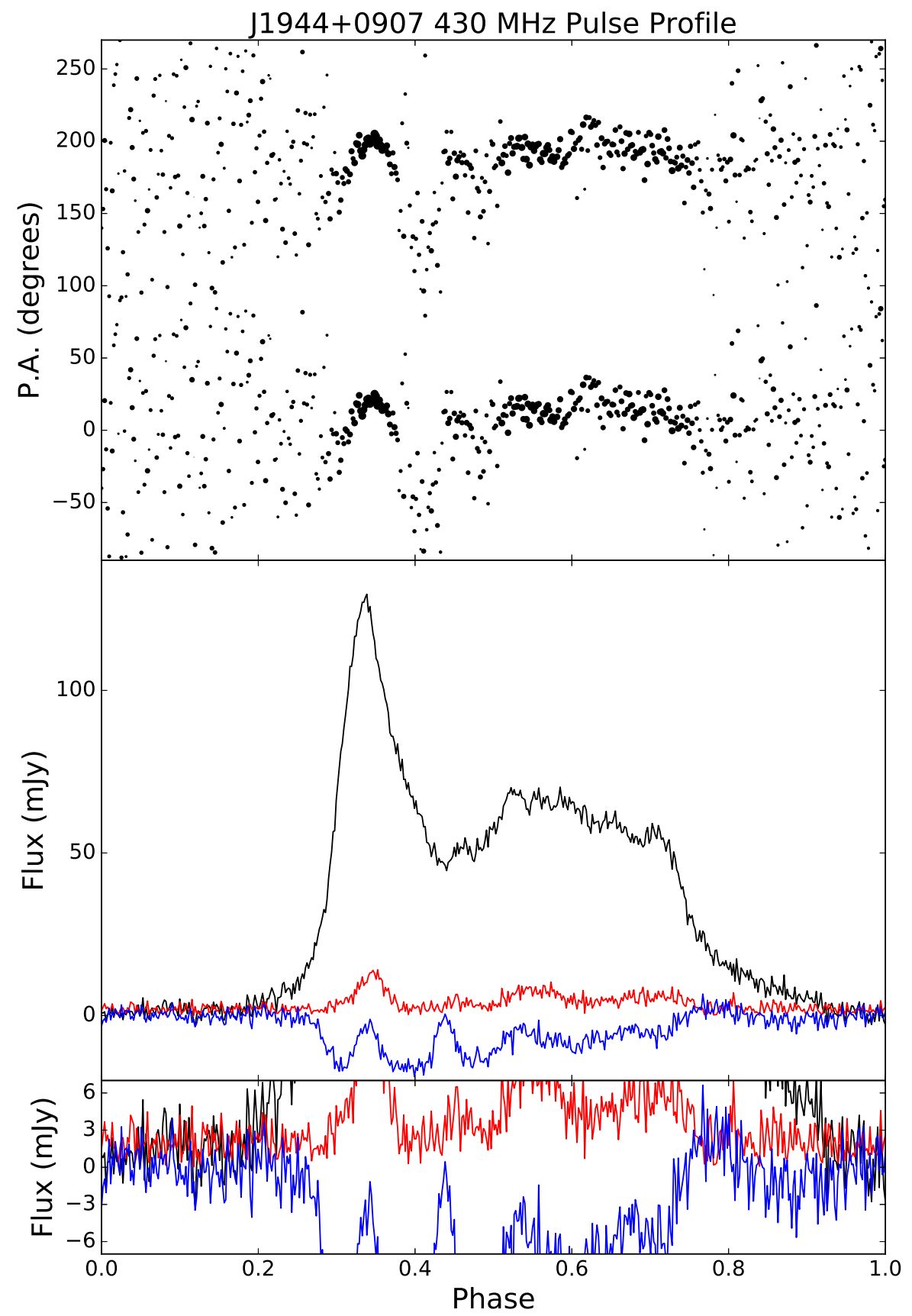

Figure A.36: Same as Figure A.1, for PSR J1944+0907 at 430 MHz. 


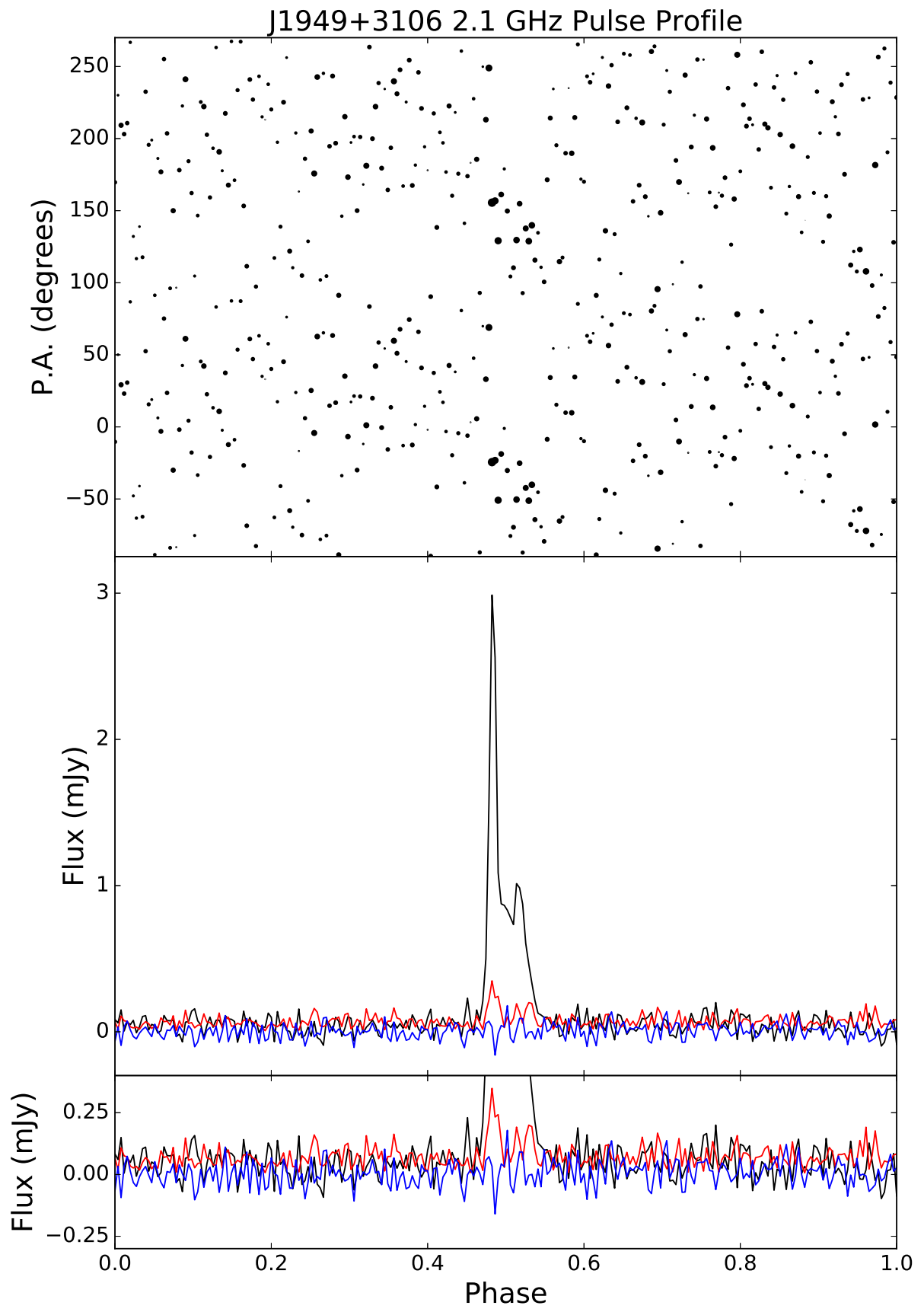

Figure A.37: Same as Figure A.1, for PSR J1949+3106 at 2.1 GHz. 


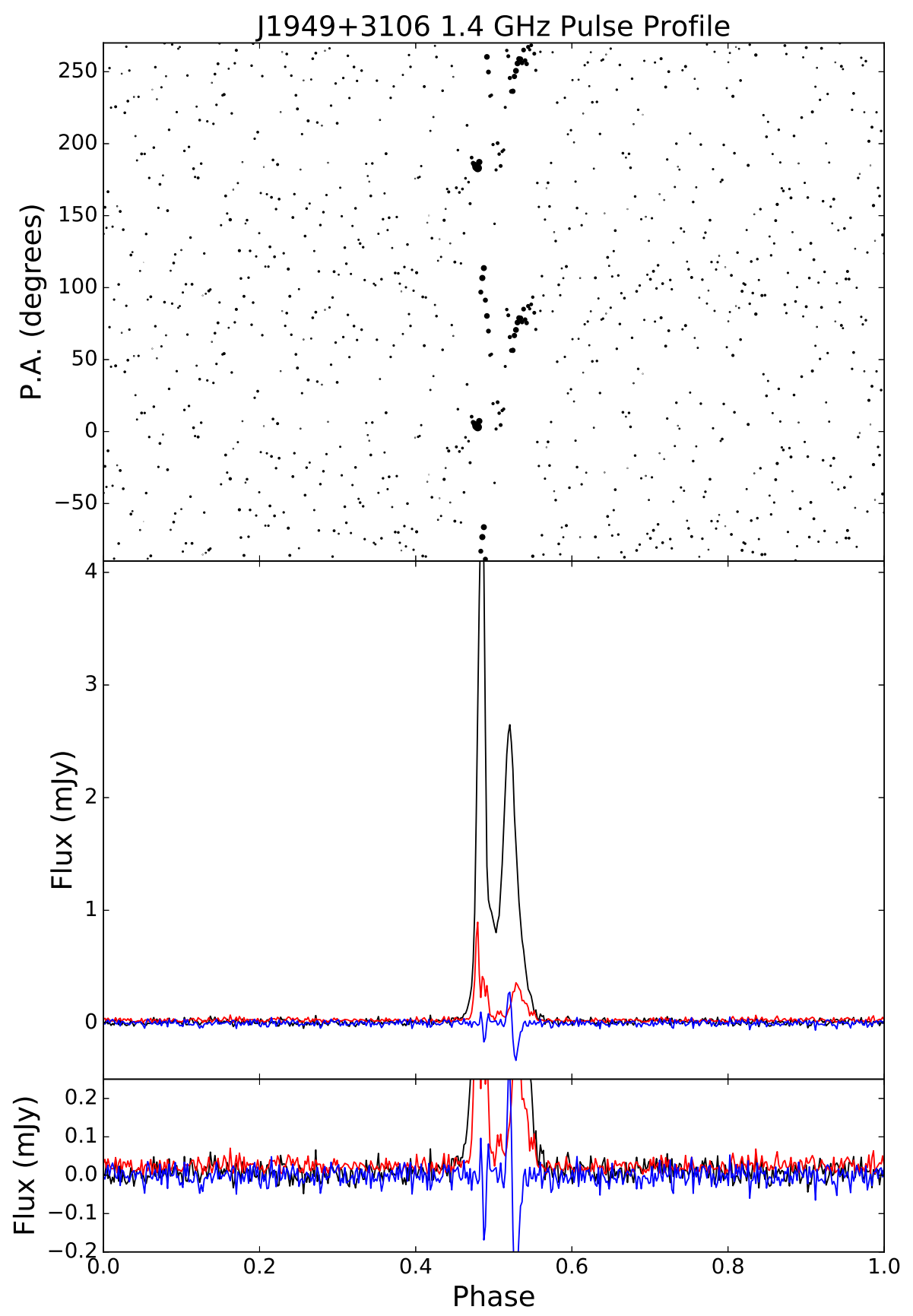

Figure A.38: Same as Figure A.1, for PSR J1949+3106 at 1.4 GHz. 


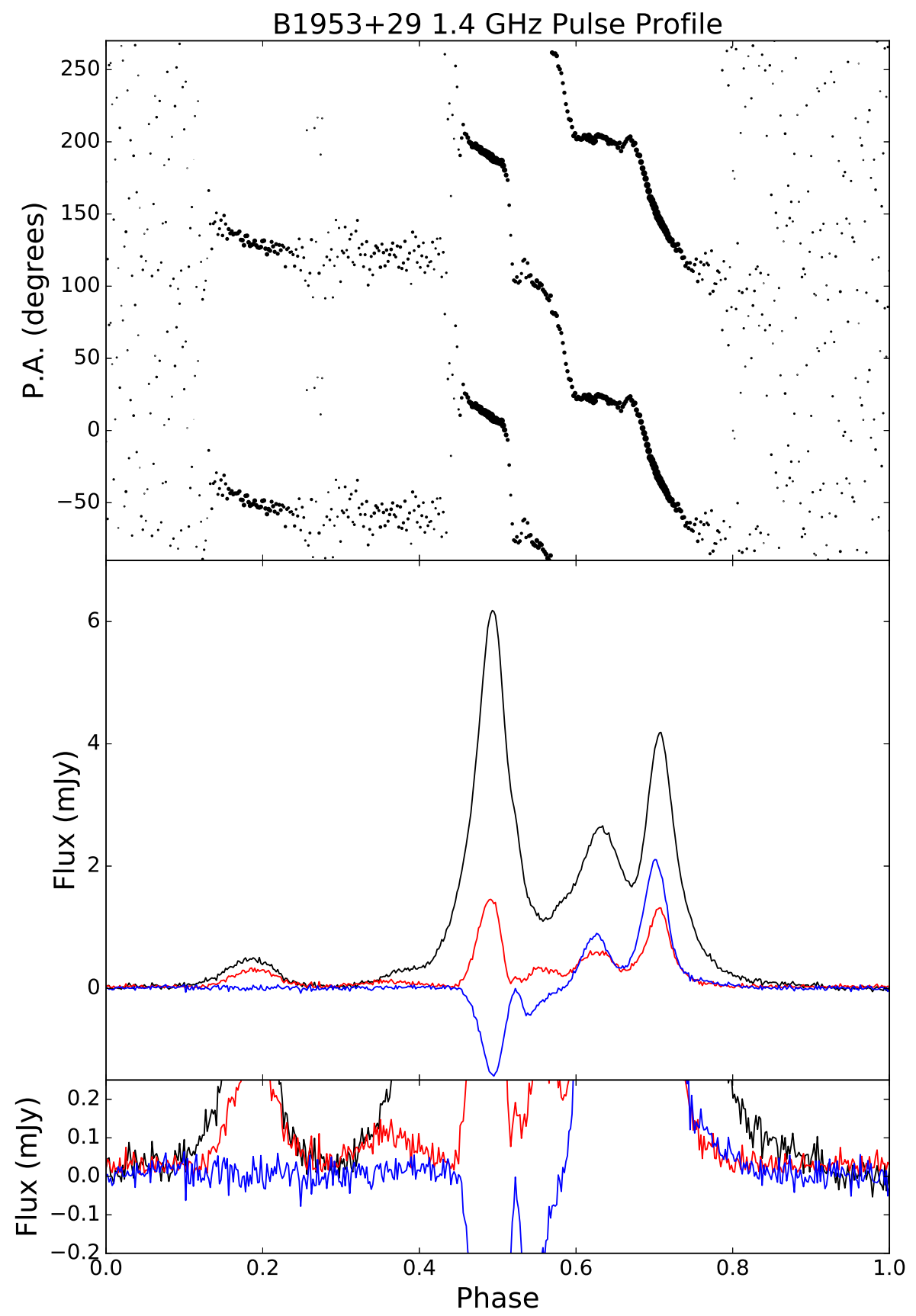

Figure A.39: Same as Figure A.1, for PSR B1953+29 at 1.4 GHz. 


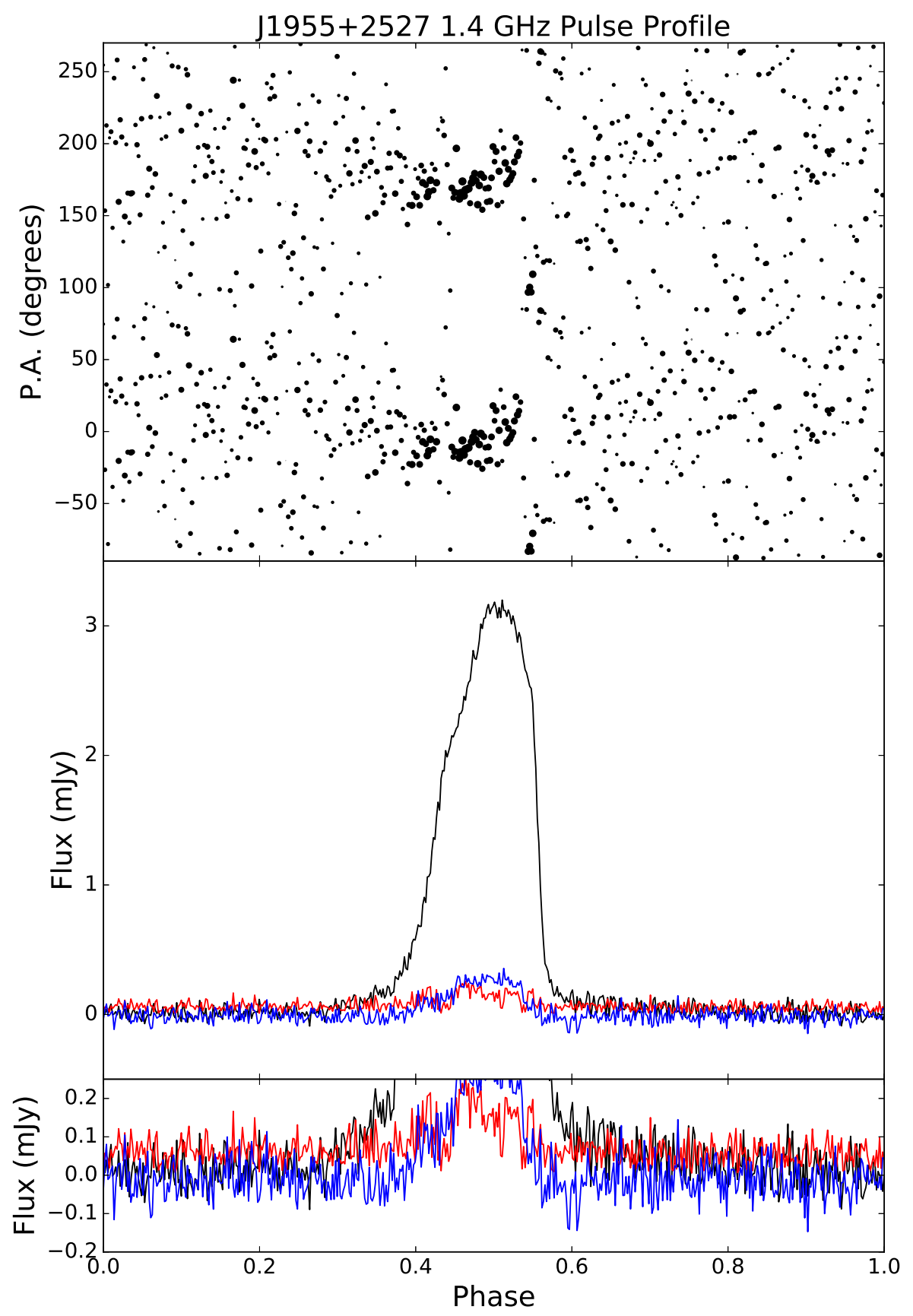

Figure A.40: Same as Figure A.1, for PSR J1955+2527 at 1.4 GHz. 


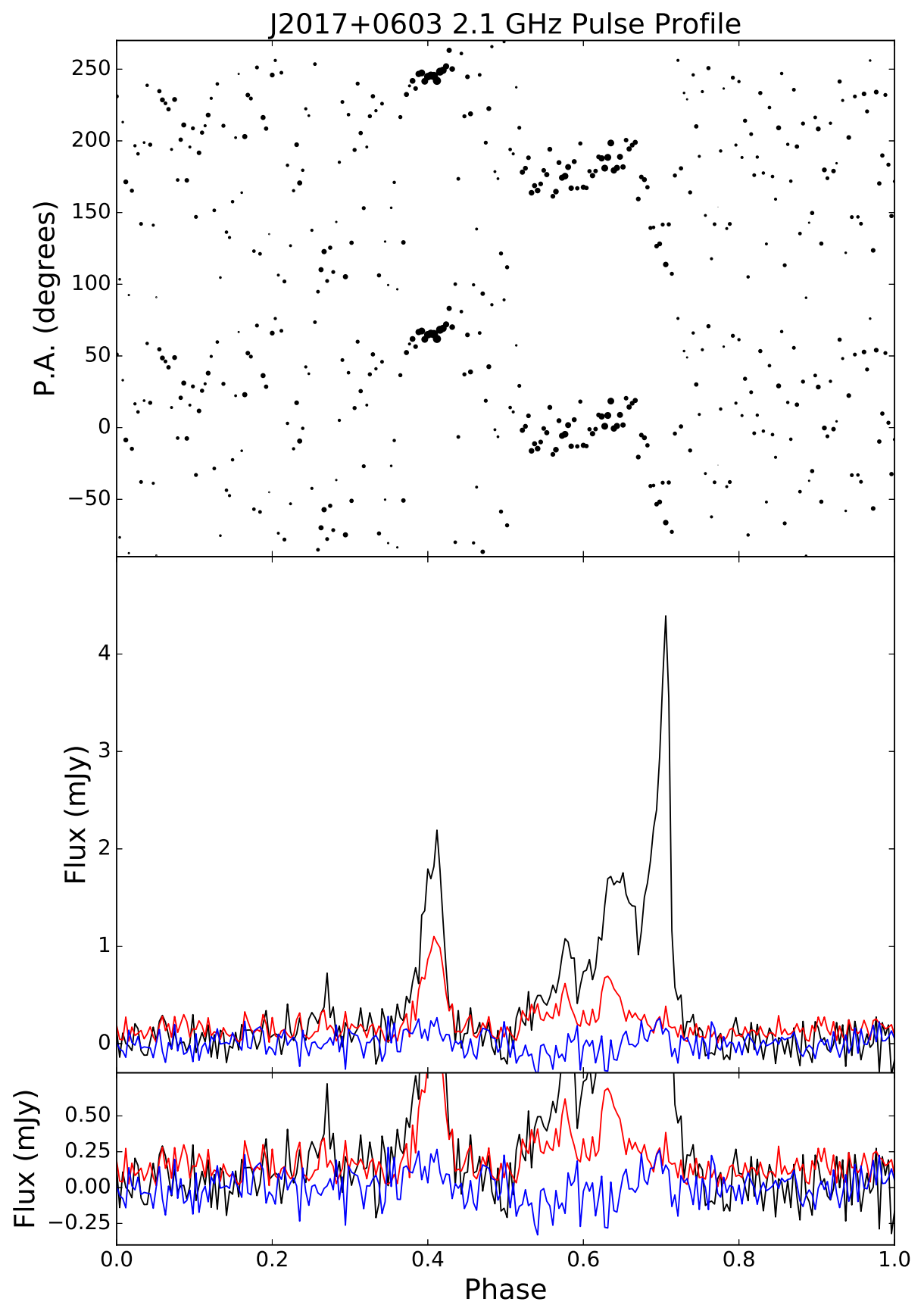

Figure A.41: Same as Figure A.1, for PSR J2017+0603 at 2.1 GHz. 


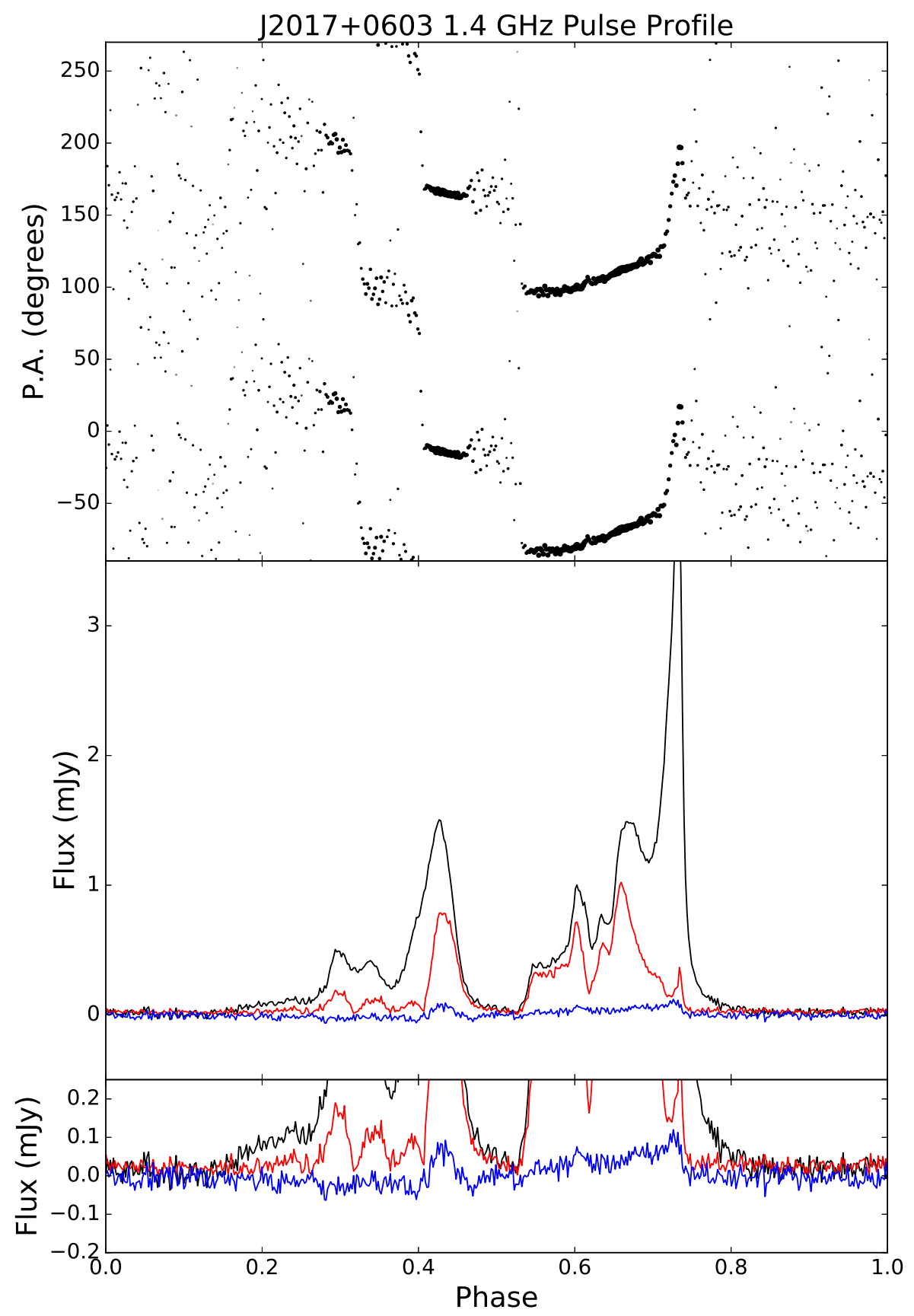

Figure A.42: Same as Figure A.1, for PSR J2017+0603 at 1.4 GHz. 


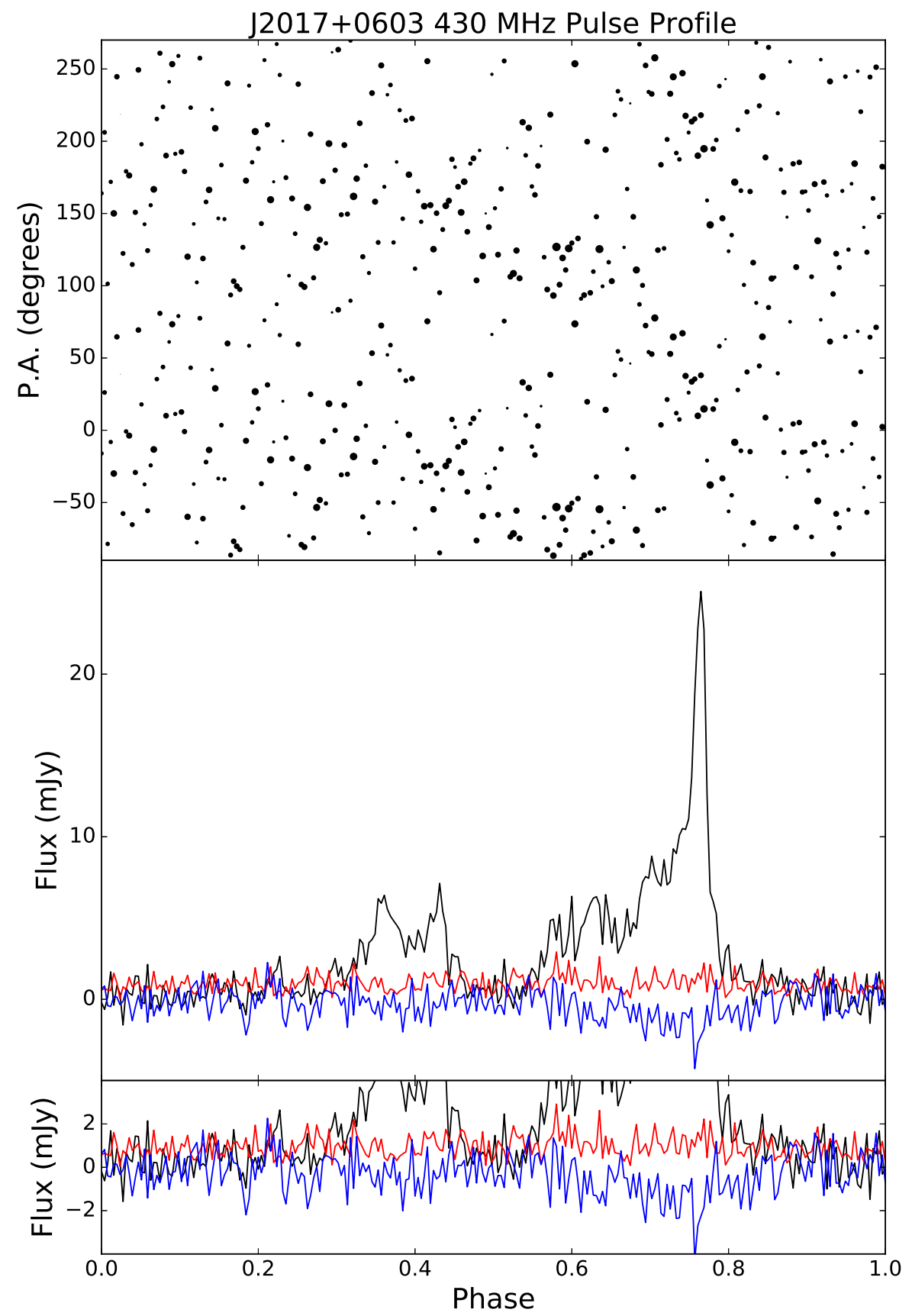

Figure A.43: Same as Figure A.1, for PSR J2017+0603 at 430 MHz. 


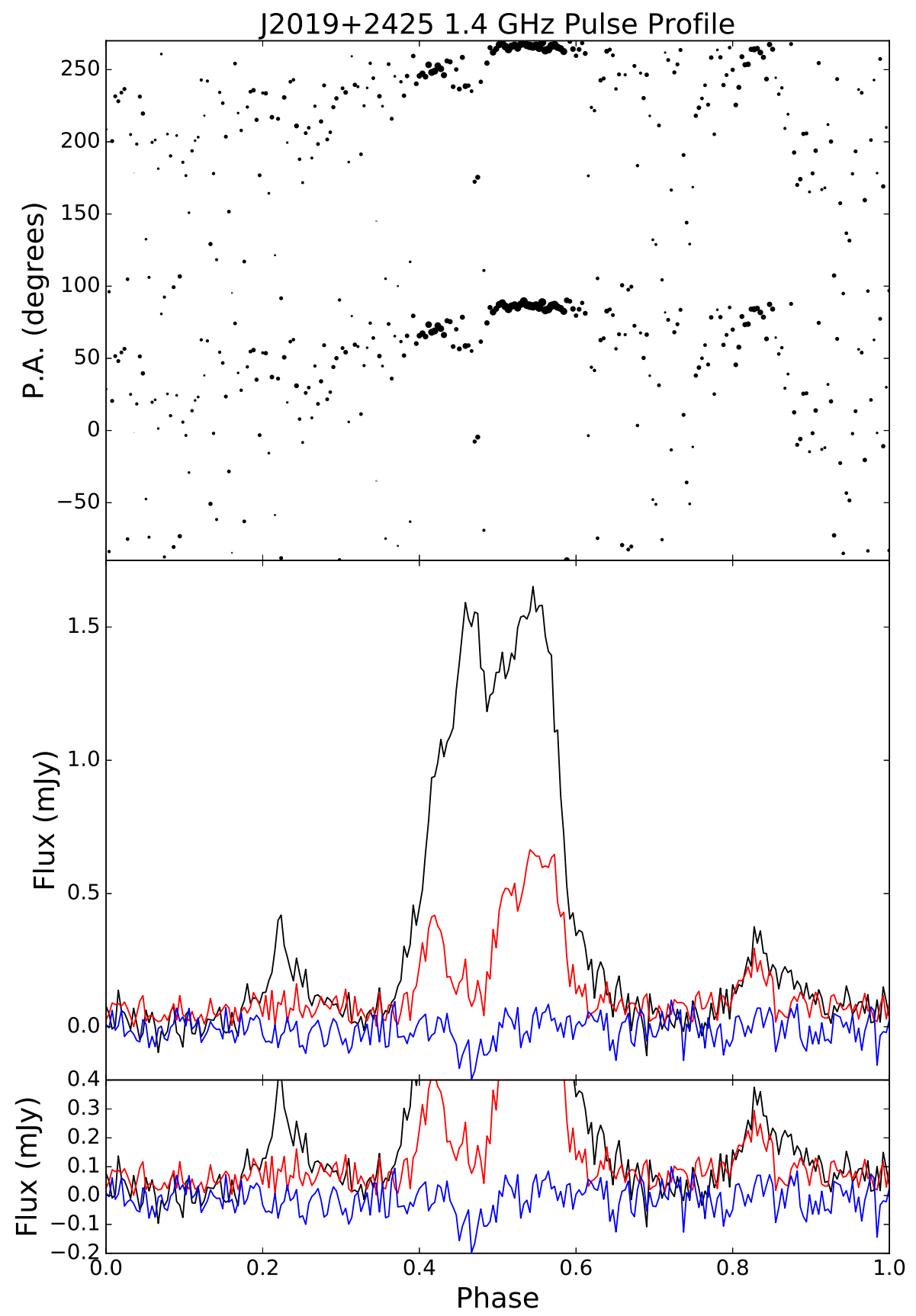

Figure A.44: Same as Figure A.1, for PSR J2019+2425 at 1.4 GHz. 


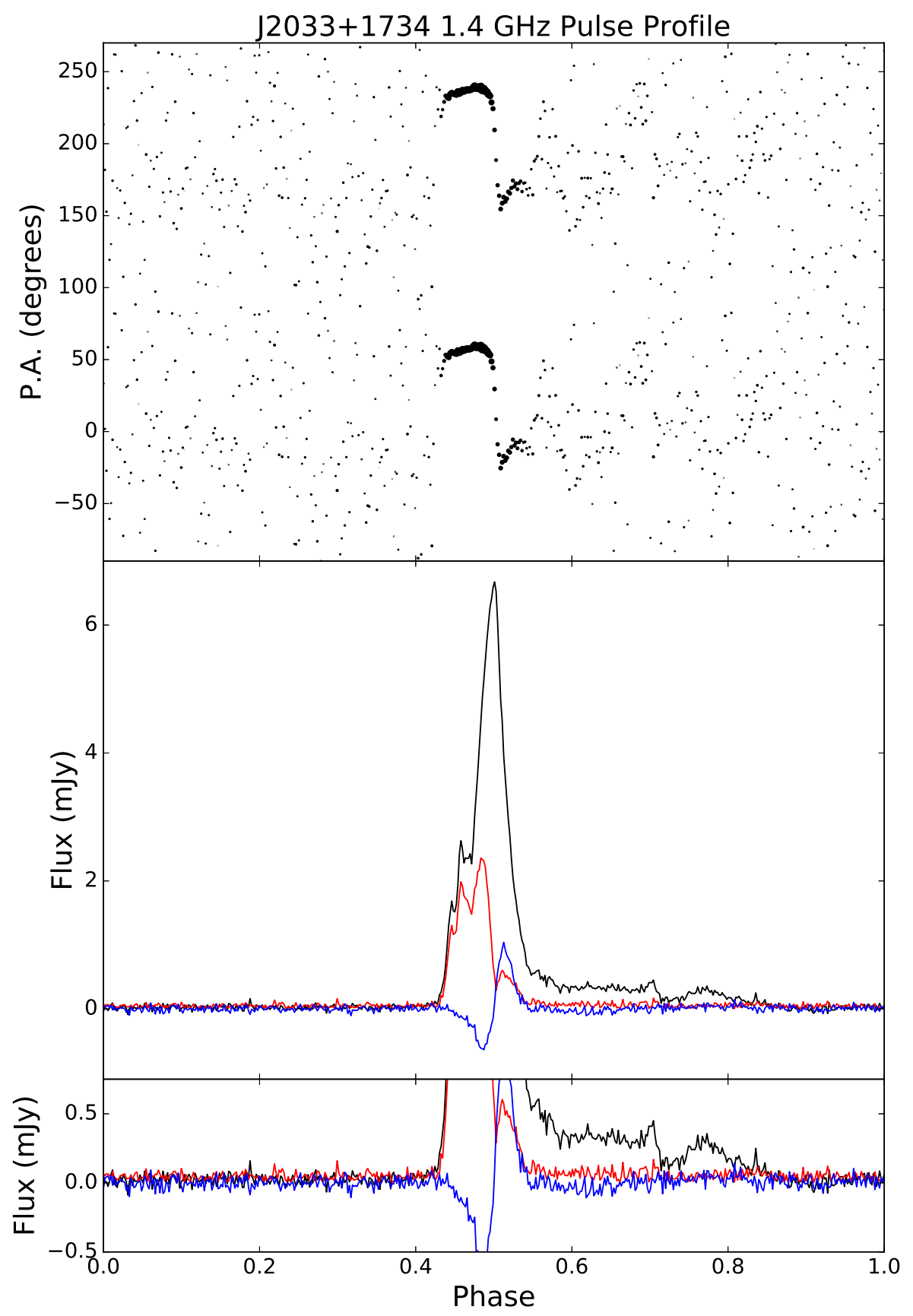

Figure A.45: Same as Figure A.1, for PSR J2033+1734 at 1.4 GHz. 


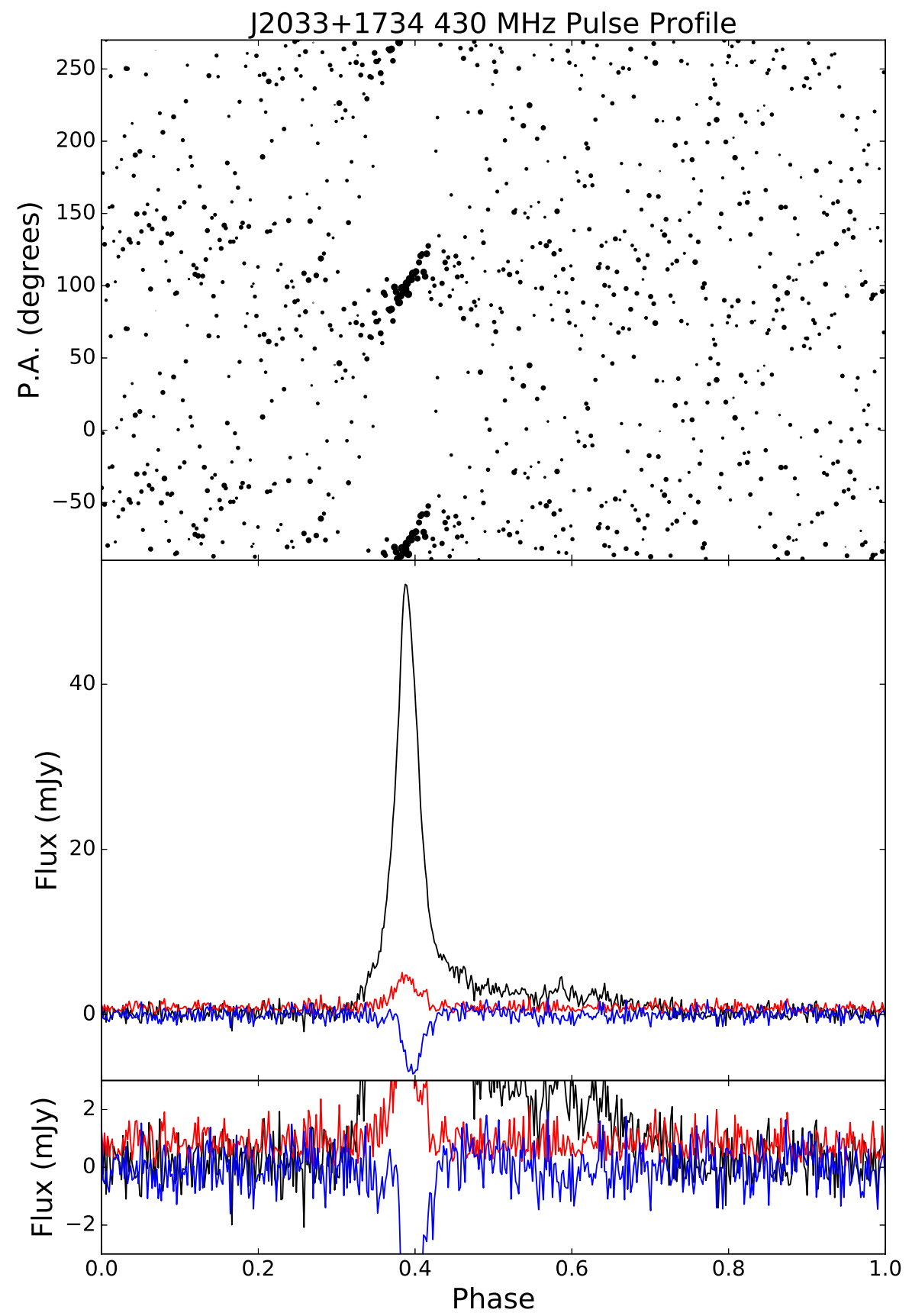

Figure A.46: Same as Figure A.1, for PSR J2033+1734 at 430 MHz. 


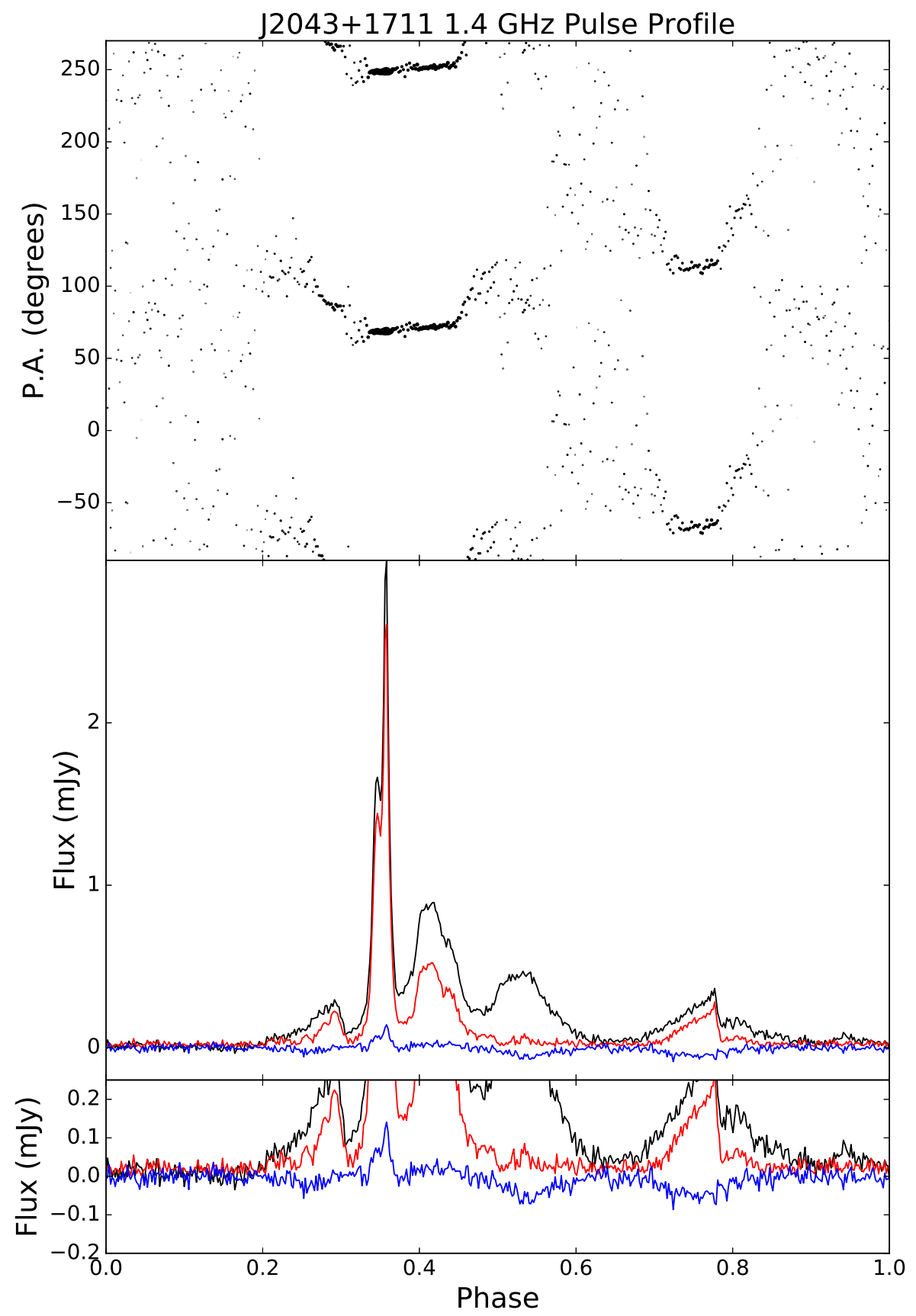

Figure A.47: Same as Figure A.1, for PSR J2043+1711 at 1.4 GHz. 


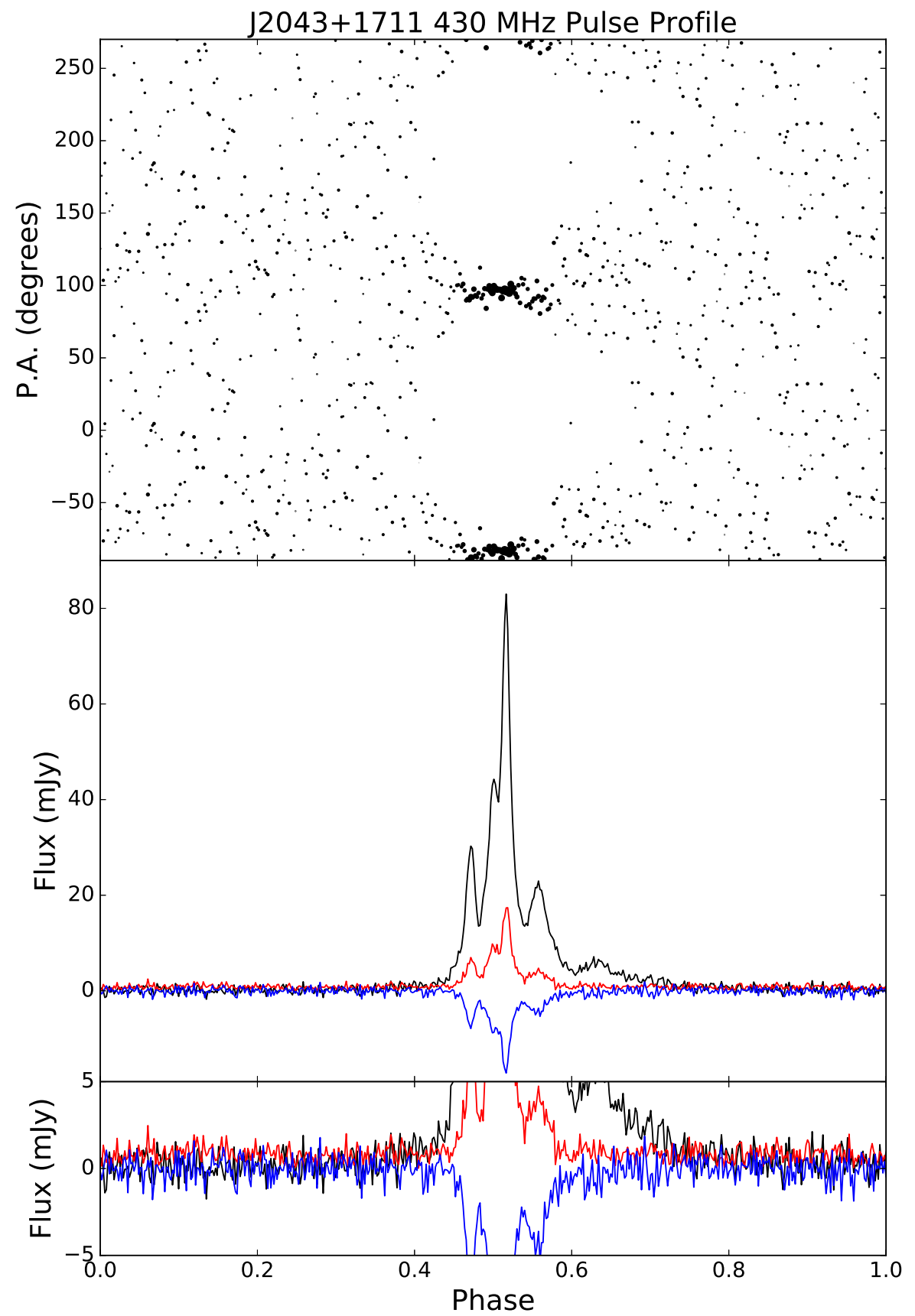

Figure A.48: Same as Figure A.1, for PSR J2043+1711 at $430 \mathrm{MHz}$. 


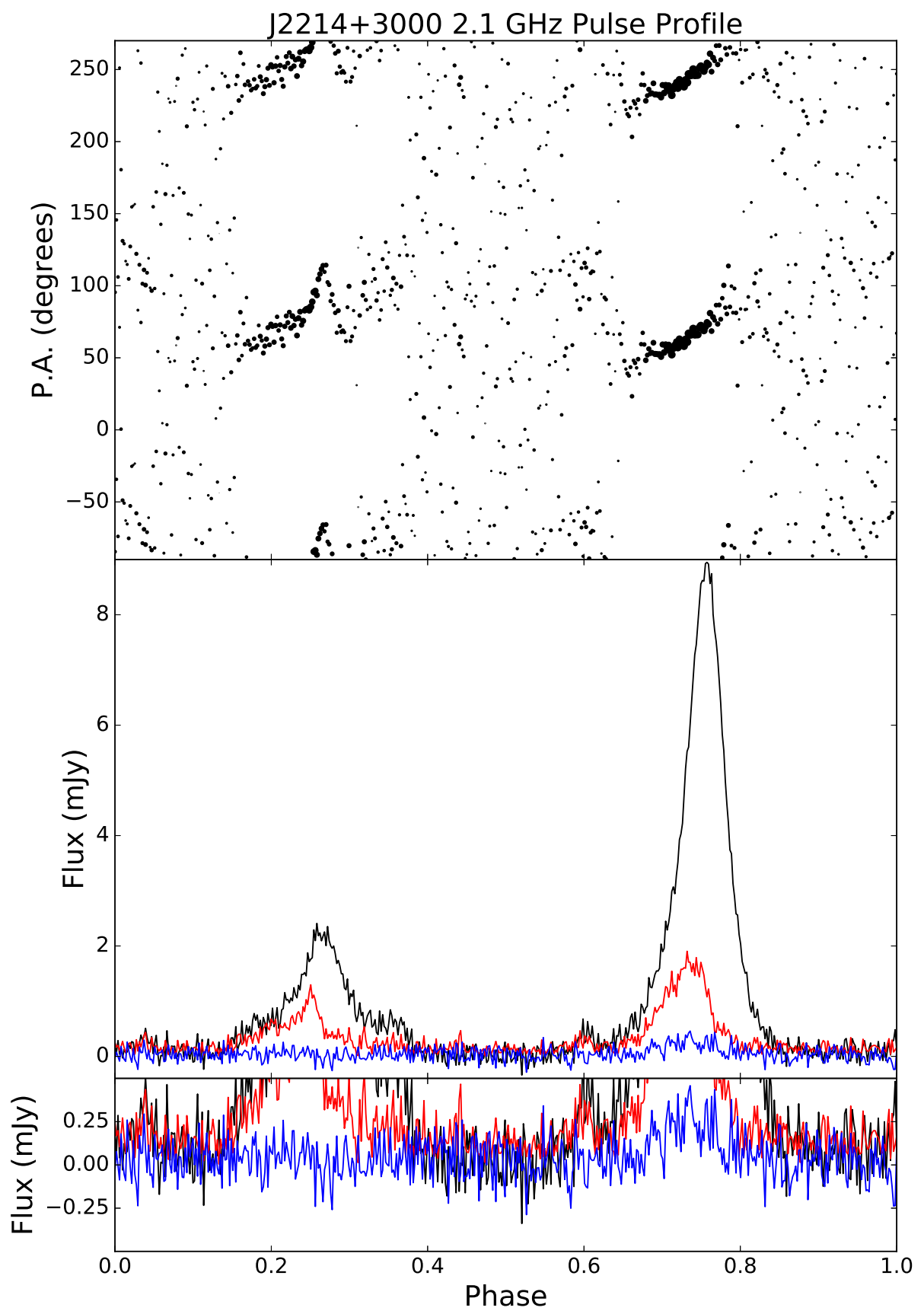

Figure A.49: Same as Figure A.1, for PSR J2214+3000 at 2.1 GHz. 


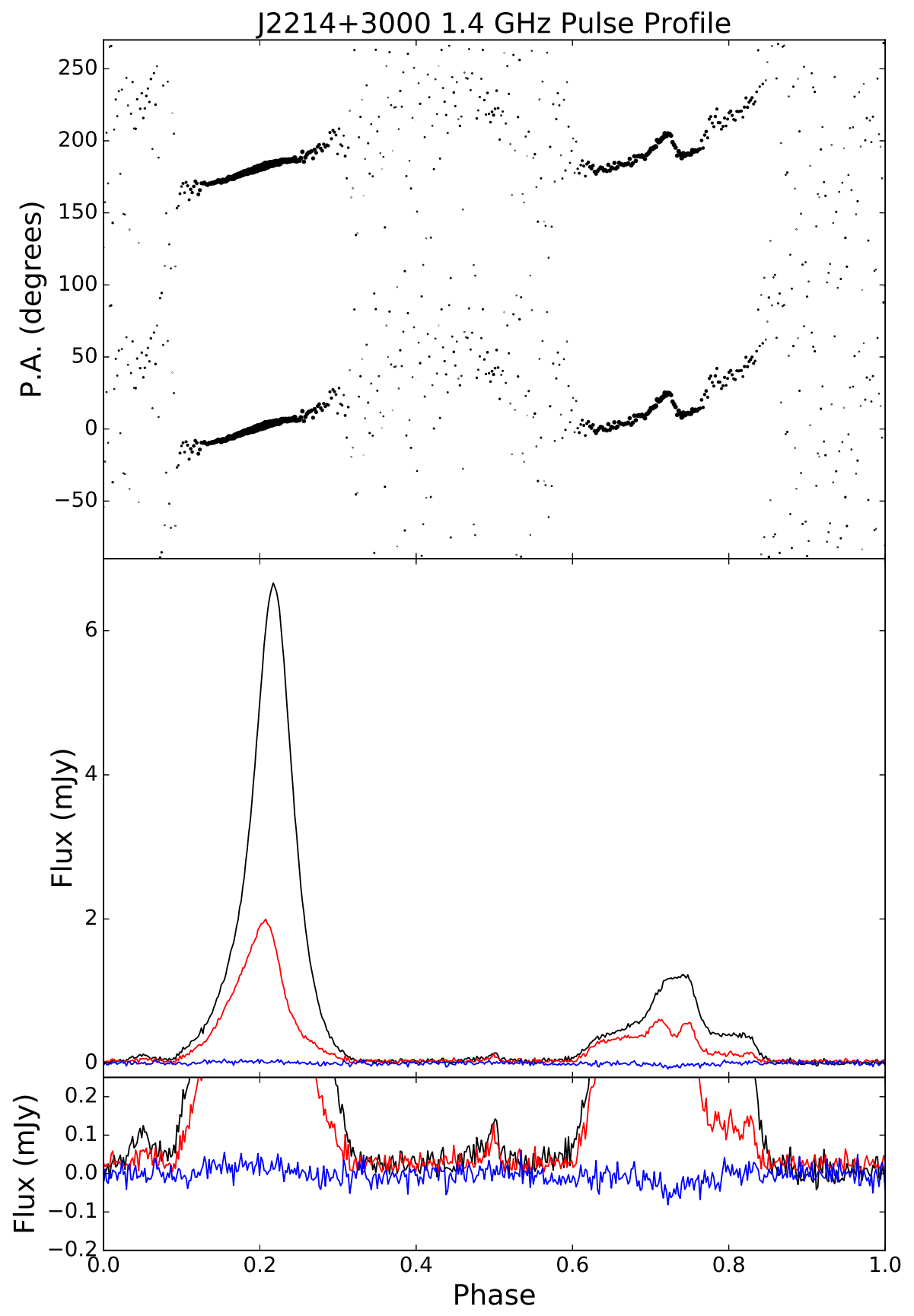

Figure A.50: Same as Figure A.1, for PSR J2214+3000 at 1.4 GHz. 


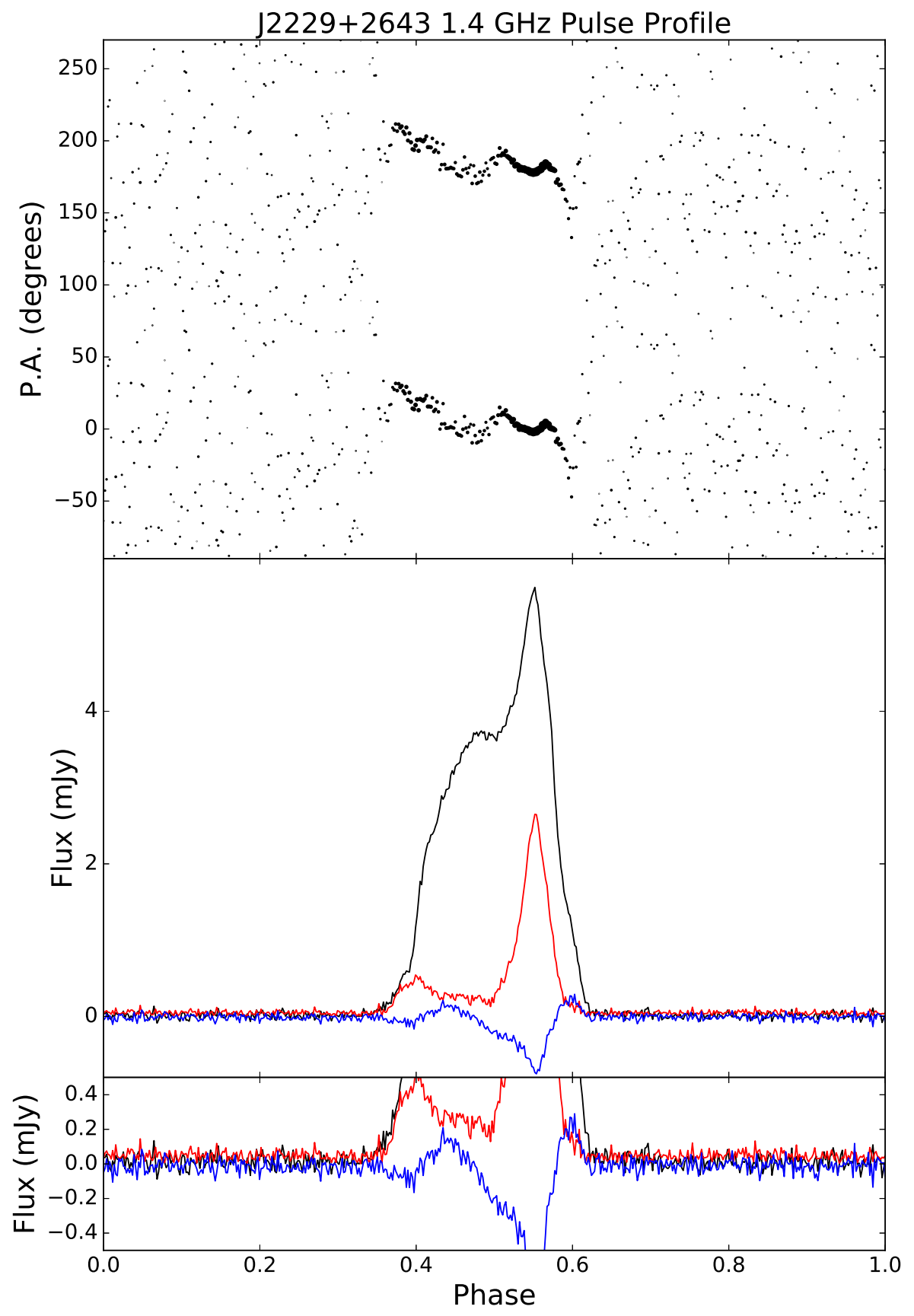

Figure A.51: Same as Figure A.1, for PSR J2229+2643 at 1.4 GHz. 


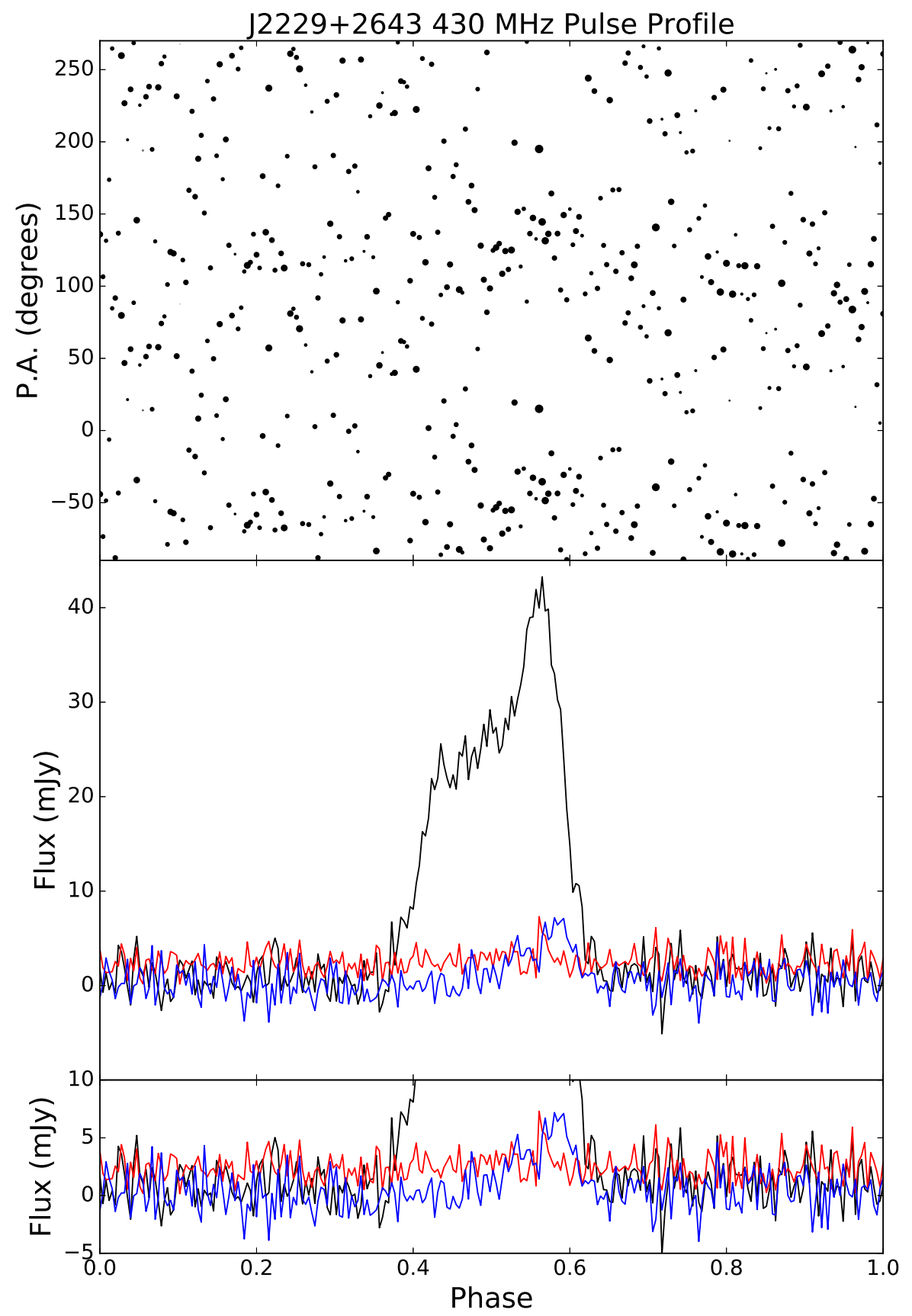

Figure A.52: Same as Figure A.1, for PSR J2229+2643 at 430 MHz. 


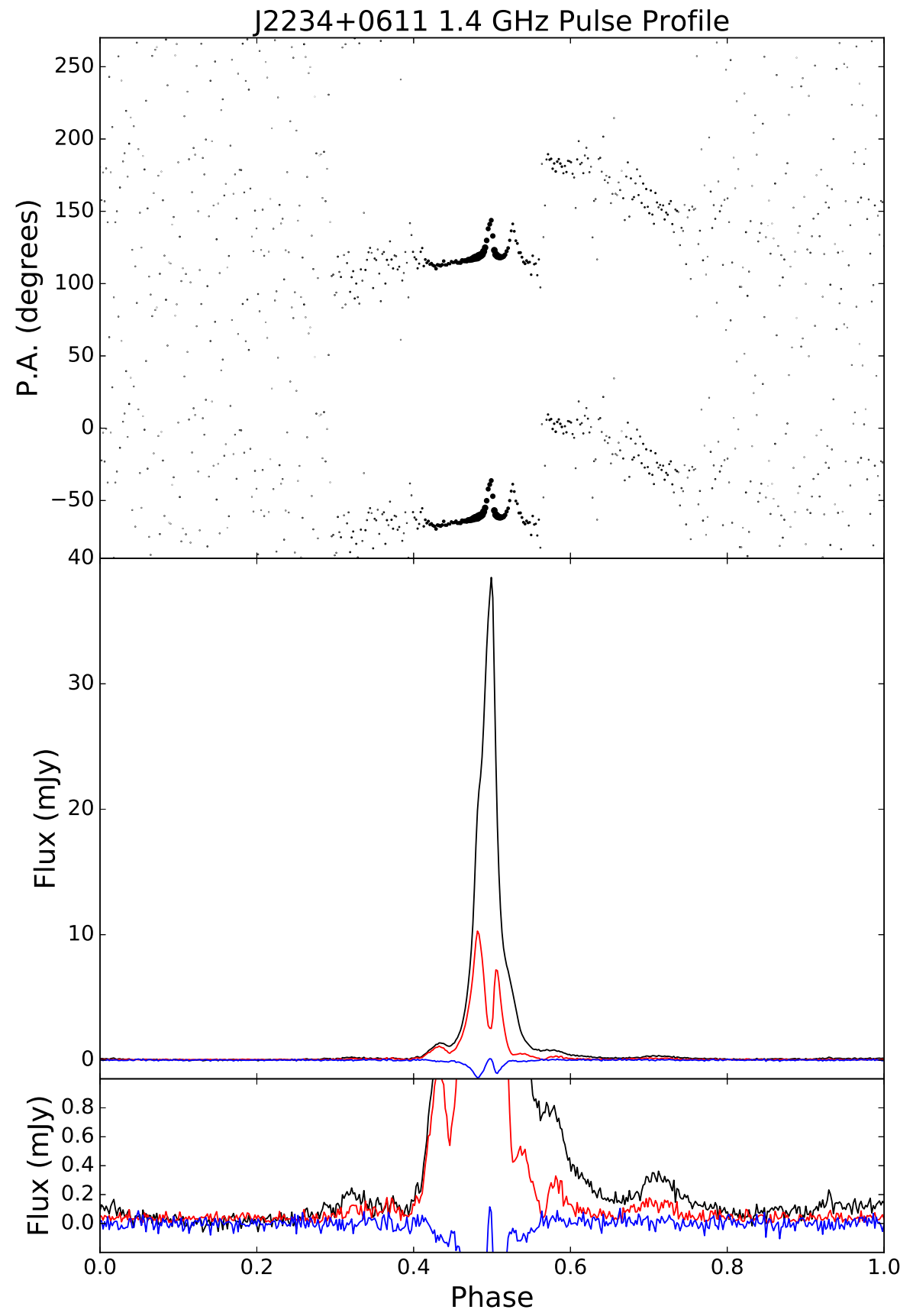

Figure A.53: Same as Figure A.1, for PSR J2234+0611 at 1.4 GHz. 


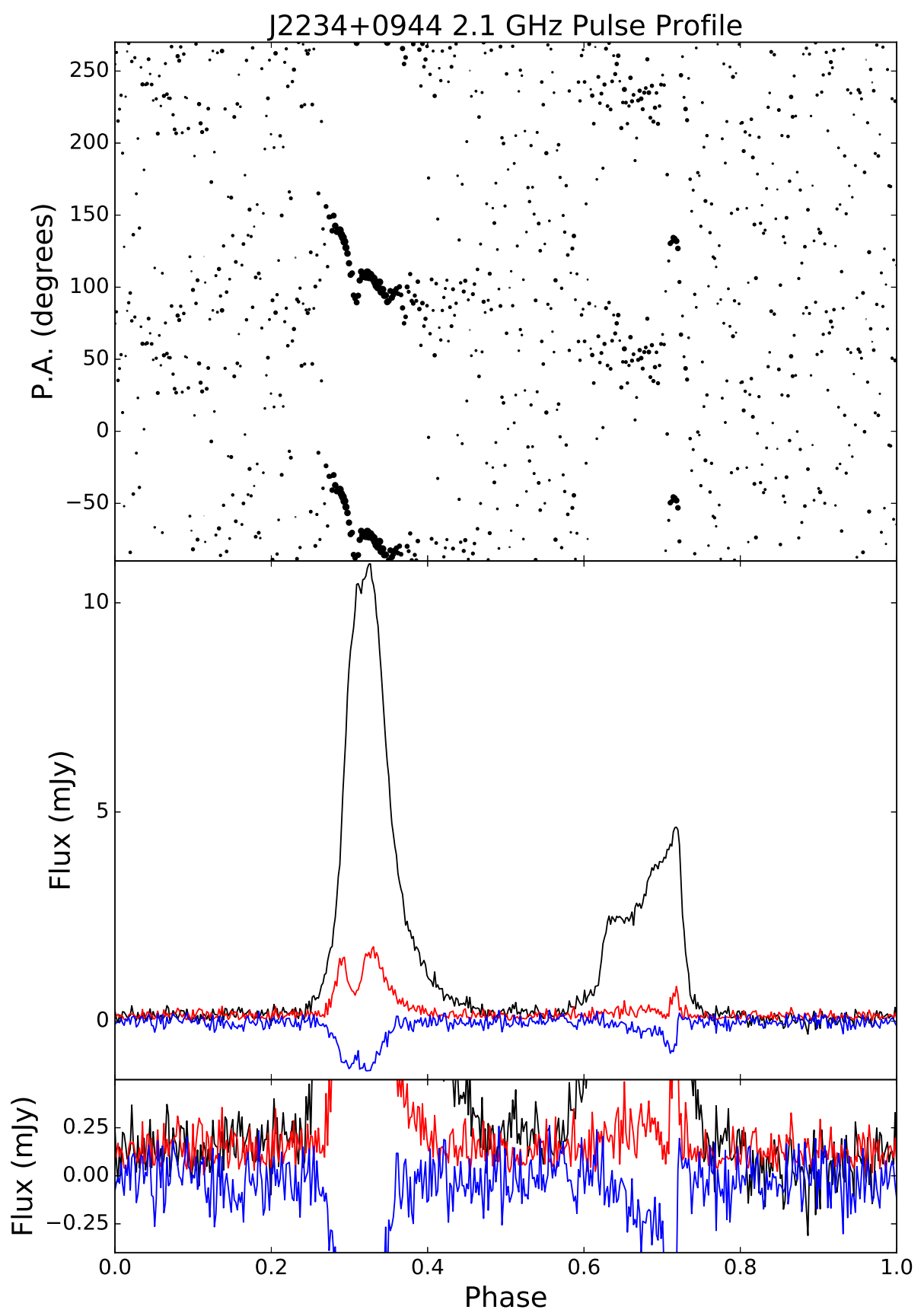

Figure A.54: Same as Figure A.1, for PSR J2234+0944 at 2.1 GHz. 


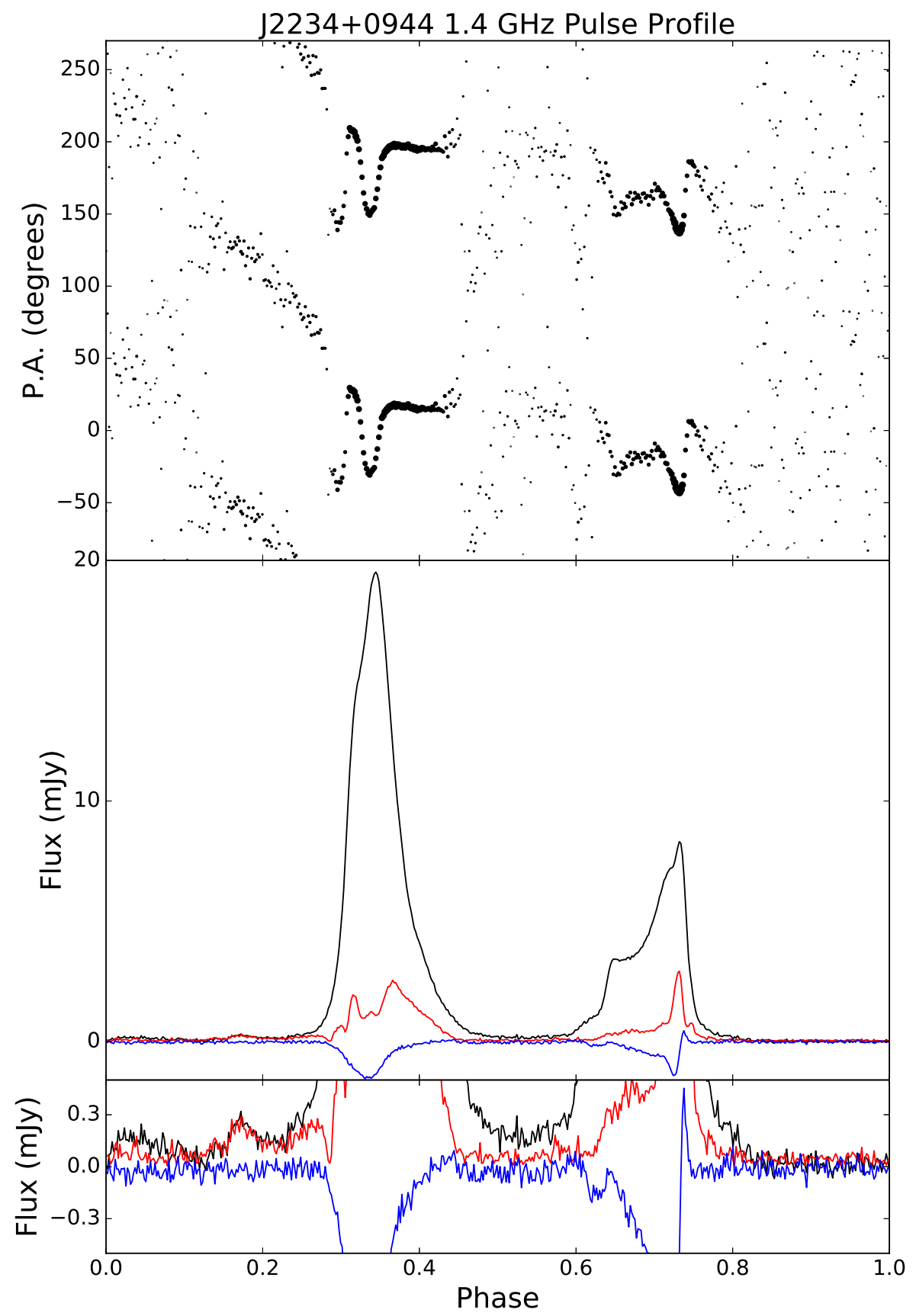

Figure A.55: Same as Figure A.1, for PSR J2234+0944 at 1.4 GHz. 


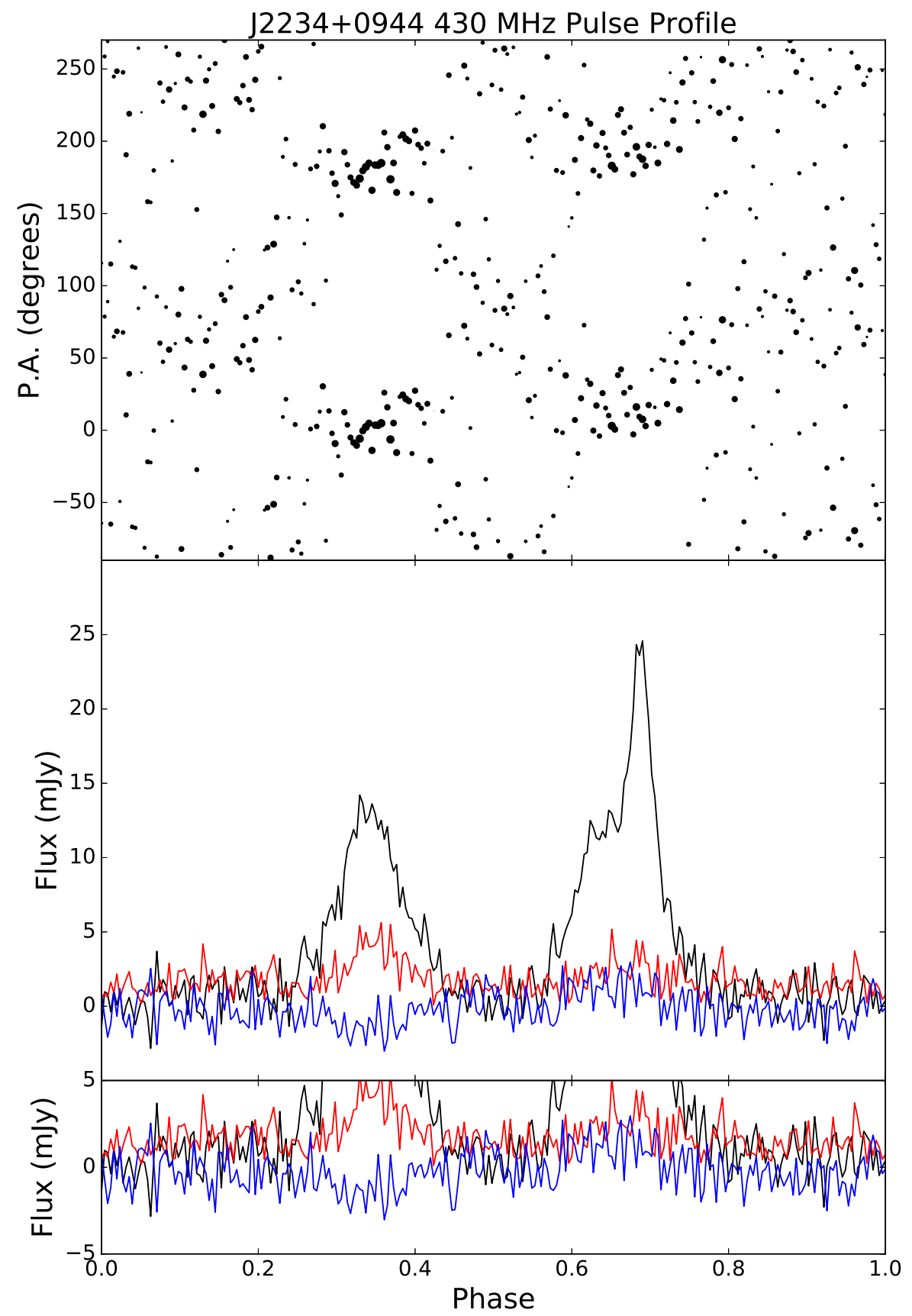

Figure A.56: Same as Figure A.1, for PSR J2234+0944 at 430 MHz. 


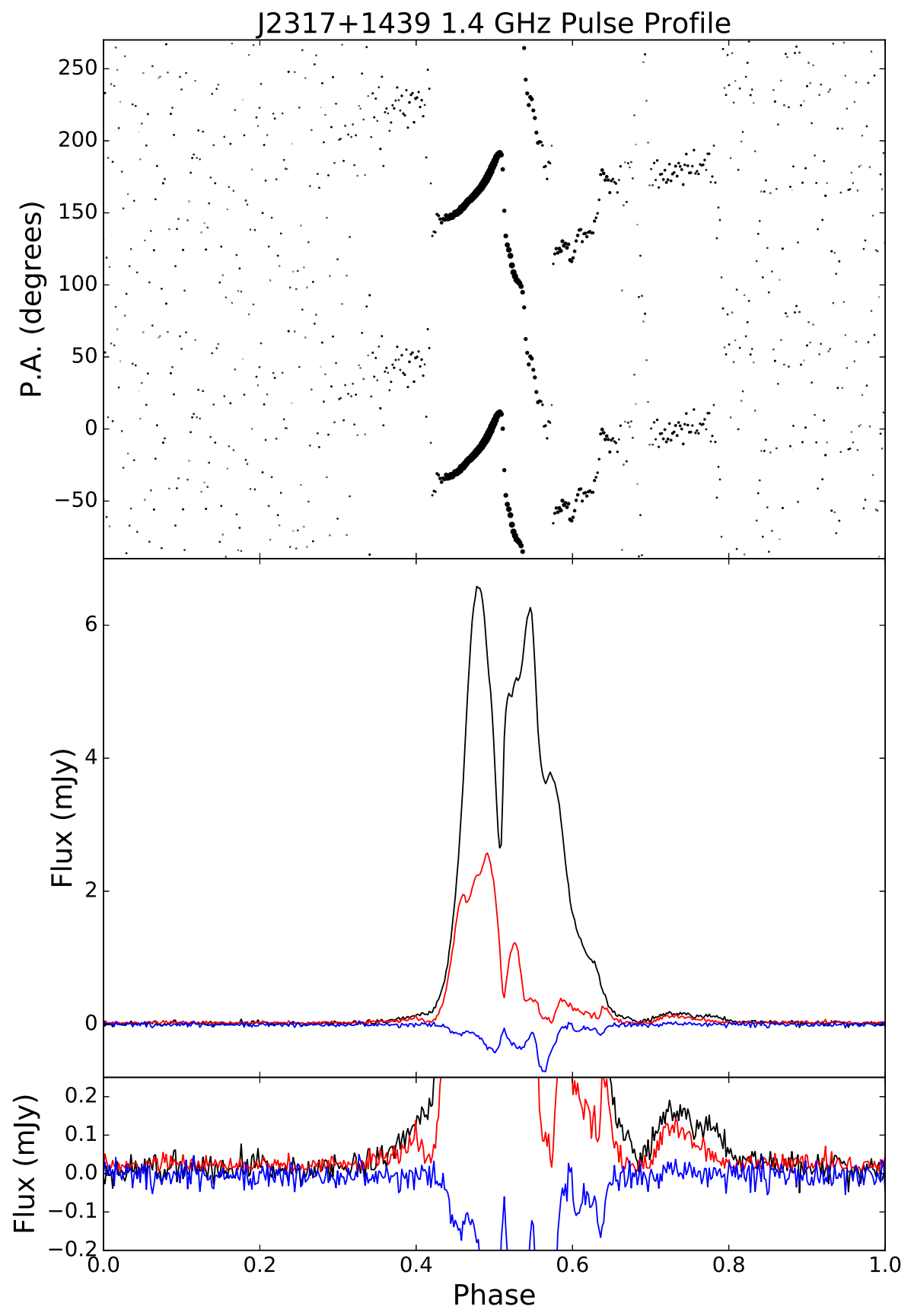

Figure A.57: Same as Figure A.1, for PSR J2317+1439 at 1.4 GHz. 


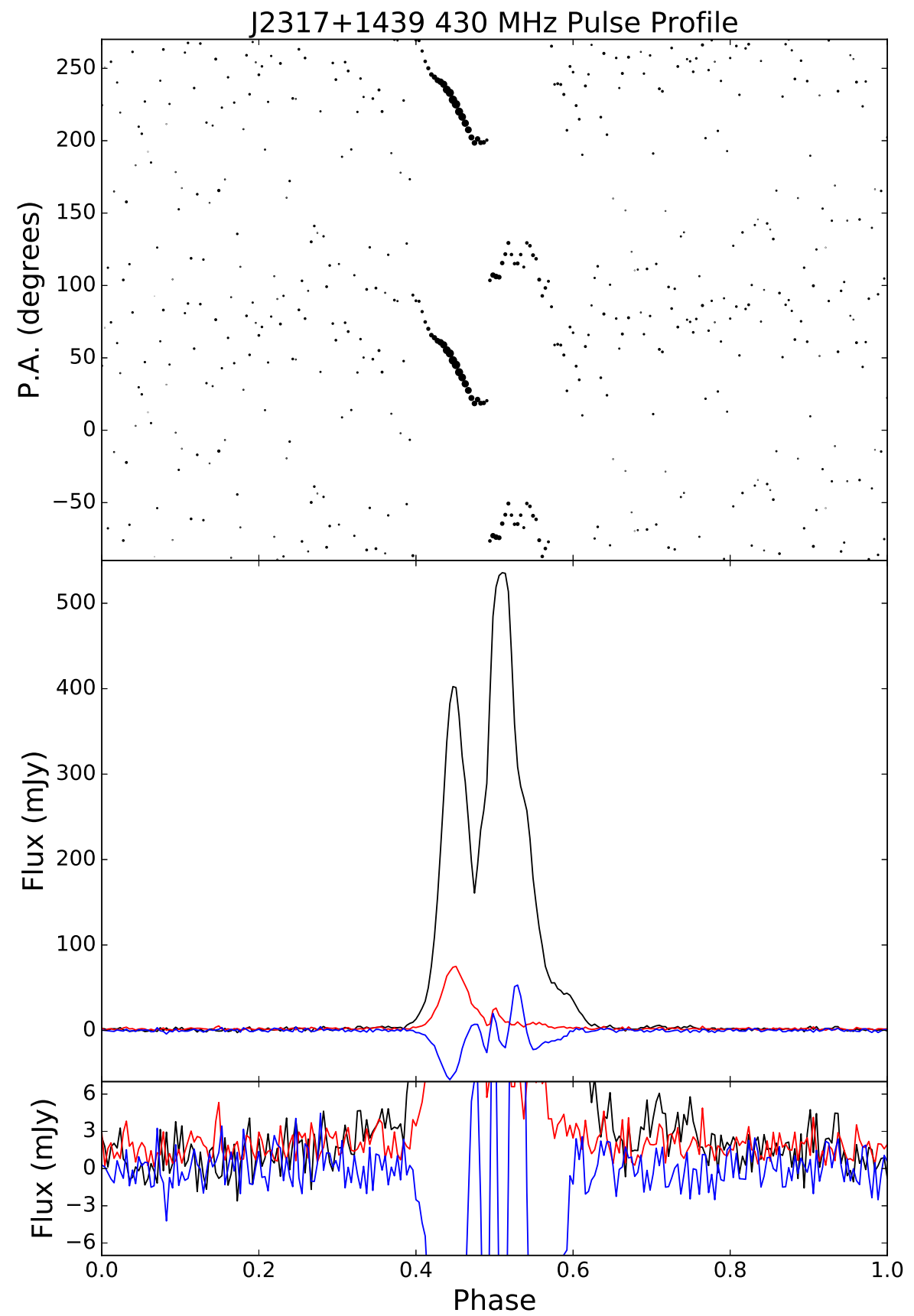

Figure A.58: Same as Figure A.1, for PSR J2317+1439 at $430 \mathrm{MHz}$. 


\section{Bibliography}

Abdo, A. A., Ackermann, M., Ajello, M., et al. 2010, A\&A, 524, A75

Alpar, M. A., Cheng, A. F., Ruderman, M. A., \& Shaham, J. 1982, Nature, 300, 728

Archibald, A. M., Kaspi, V. M., Bogdanov, S., et al. 2010, ApJ, 722, 88

Archibald, A. M., Stairs, I. H., Ransom, S. M., et al. 2009, Science, 324, 1411

Arons, J., \& Tavani, M. 1993, Astrophys. J., 403, 249

Arzoumanian, Z., Fruchter, A. S., \& Taylor, J. H. 1994, Astrophys. J., 426, L85

Backer, D. C., Rankin, J. M., \& Campbell, D. B. 1975, Astrophys. J., 197, 481

Baring, M. G., \& Harding, A. K. 1997, ApJ, 482, 372

Baring, M. G., \& Harding, A. K. 1998, Astrophys. J. Lett., 507, L55

Bhattacharya, D., Wijers, R. A. M. J., Hartman, J. W., \& Verbunt, F. 1992, Astron. Astrophys., 254, 198

Bisnovatyi-Kogan, G. S., \& Komberg, B. V. 1974, Sov. Astron., 18, 217

Bogdanov, S., Archibald, A. M., Hessels, J. W. T., et al. 2011, ApJ, 742, 97

Bogdanov, S., Grindlay, J. E., \& van den Berg, M. 2005, ApJ, 630, 1029

Bogdanov, S., Rybicki, G. B., \& Grindlay, J. E. 2007, ApJ, 670, 668

Bogdanov, S. B. 2008, PhD thesis, Harvard University 
Bond, H. E., White, R. L., Becker, R. H., \& O’Brien, M. S. 2002, PASP, 114, 1359

Boriakoff, V., Buccheri, R., \& Fauci, F. 1983, Nature, 304, 417

Boyles, J., Lynch, R. S., Ransom, S. M., et al. 2013, ApJ, 763, 80

Breton, R. P., van Kerkwijk, M. H., Roberts, M. S. E., et al. 2013, ApJ, 769, 108

Camilo, F., Nice, D. J., Shrauner, J. A., \& Taylor, J. H. 1996a, Astrophys. J., 469, 819

Camilo, F., Nice, D. J., \& Taylor, J. H. 1993, Astrophys. J. Lett., 412, L37

—. 1996b, Astrophys. J., 461, 812

Champion, D. J., Lorimer, D. R., McLaughlin, M. A., et al. 2005, Mon. Not. R. Astron. Soc., 363, 929

Chen, K., \& Ruderman, M. 1993, Astrophys. J., 402, 264

Comella, J. M., Craft, H. D., Lovelace, R. V. E., Sutton, J. M., \& Tyler, G. L. 1969, Nature, 221, 453

Cordes, J. M., \& Lazio, T. J. W. 2002, astro-ph/0207156

Cordes, J. M., Shannon, R. M., \& Stinebring, D. R. 2016, ApJ, 817, 16

Craig, H. A. 2014, ApJ, 790, 102

Dai, S., Hobbs, G., Manchester, R. N., et al. 2015, MNRAS, 449, 3223 
D’Amico, N., Possenti, A., Manchester, R. N., et al. 2001, Astrophys. J. Lett., 561, L89. http://adsabs.harvard.edu/cgi-bin/nph-bib_query?bibcode= 2001ApJ . . . 561L . .89D\&db_key=AST

Deller, A. T., Archibald, A. M., Brisken, W. F., et al. 2012, ApJL, 756, L25

Demorest, P. B., Ferdman, R. D., Gonzalez, M. E., et al. 2013, ApJ, 762, 94

Deneva, J. S., Freire, P. C. C., Cordes, J. M., et al. 2012, ApJ, 757, 89

Dickey, J. M., \& Lockman, F. J. 1990, Ann. Rev. Astron. Astrophys., 28, 215

Espinoza, C. M., Guillemot, L., Çelik, Ö., et al. 2013, MNRAS, 430, 571

Foster, R. S., Cadwell, B. J., Wolszczan, A., \& Anderson, S. B. 1995, Astrophys. J., 454,826

Freire, P. C. C., Wex, N., Esposito-Farèse, G., et al. 2012, MNRAS, 423, 3328

Fruchter, A. S., Gunn, J. E., Lauer, T. R., \& Dressler, A. 1988a, Nature, 334, 686

Fruchter, A. S., Stinebring, D. R., \& Taylor, J. H. 1988b, Nature, 333, 237

Fruchter, A. S., Berman, G., Bower, G., et al. 1990, Astrophys. J., 351, 642

Gentile, P. A., Roberts, M. S. E., McLaughlin, M. A., et al. 2014, ApJ, 783, 69

Goldreich, P., \& Julian, W. H. 1969, Astrophys. J., 157, 869

Gonzalez, M. E., Stairs, I. H., Ferdman, R. D., et al. 2011, ApJ, 743, 102

Green, G. M., Schlafly, E. F., Finkbeiner, D. P., et al. 2015, ApJ, 810, 25 
Grindlay, J. E., Camilo, F., Heinke, C. O., et al. 2002, Astrophys. J., 581, 470

Guillemot, L., Freire, P. C. C., Cognard, I., et al. 2012a, MNRAS, 422, 1294

Guillemot, L., Johnson, T. J., Venter, C., et al. 2012b, ApJ, 744, 33

Güver, T., \& Özel, F. 2009, MNRAS, 400, 2050

Han, J. L., Manchester, R. N., Berkhuijsen, E. M., \& Beck, R. 1997, Astron. Astrophys., 322,98

Han, J. L., Manchester, R. N., Lyne, A. G., Qiao, G. J., \& van Straten, W. 2006, ApJ, 642, 868

He, C., Ng, C.-Y., \& Kaspi, V. M. 2013, ApJ, 768, 64

Heiles, C. 2002, in Astronomical Society of the Pacific Conference Series, Vol. 278, Single-Dish Radio Astronomy: Techniques and Applications, ed. S. Stanimirovic, D. Altschuler, P. Goldsmith, \& C. Salter, 131-152

Hewish, A., Bell, S. J., Pilkington, J. D. H., Scott, P. F., \& Collins, R. A. 1968, Nature, 217, 709

Huang, H. H., \& Becker, W. 2007, A\&A, 463, L5

Huang, R. H. H., Kong, A. K. H., Takata, J., et al. 2012, ApJ, 760, 92

Jacoby, B. A. 2005, PhD thesis, California Institute of Technology, California, USA

Jacoby, B. A., Bailes, M., Ord, S. M., Knight, H. S., \& Hotan, A. W. 2007, ApJ, 656,408 
Johnston, S., \& Weisberg, J. M. 2006, MNRAS, 368, 1856

Jones, M. L., McLaughlin, M. A., Lam, M. T., et al. 2017, ApJ, 841, 125

Kalberla, P. M. W., Burton, W. B., Hartmann, D., et al. 2005, A\&A, 440, 775

Kennel, C. F., \& Coroniti, F. V. 1984, Astrophys. J., 283, 694

Komesaroff, M. M. 1970, Nature, 225, 612

Kramer, M., Xilouris, K. M., Lorimer, D. R., et al. 1998, Astrophys. J., 501, 270

Kuiper, L., Hermsen, W., \& Stappers, B. 2003, in Pulsars, AXPs and SGRs Observed with BeppoSAX and Other Observatories, ed. G. Cusumano, E. Massaro, \& T. Mineo, $31-36$

Kuiper, L., Hermsen, W., Verbunt, F., et al. 2000, A\&A, 359, 615

Lewandowski, W., Wolszczan, A., Feiler, G., Konacki, M., \& Sołtysiński, T. 2004, Astrophys. J., 600, 905

Lommen, A. N., Zepka, A., Backer, D. C., et al. 2000, Astrophys. J., 545, 1007

Lorimer, D. R., \& Kramer, M. 2005, Handbook of Pulsar Astronomy (Cambridge University Press)

Lorimer, D. R., McLaughlin, M. A., Champion, D. J., \& Stairs, I. H. 2007, MNRAS, 379,282

Lorimer, D. R., Faulkner, A. J., Lyne, A. G., et al. 2006, MNRAS, 372, 777 
Lynch, R. S., Boyles, J., Ransom, S. M., et al. 2013, ApJ, 763, 81

Lyne, A., \& Graham-Smith, F. 2012, Pulsar Astronomy

Lyne, A. G., \& Manchester, R. N. 1988, Mon. Not. R. Astron. Soc., 234, 477

Manchester, R. N., Hobbs, G. B., Teoh, A., \& Hobbs, M. 2005, Astron. J., 129, 1993

Manchester, R. N., Taylor, J. H., \& Huguenin, G. R. 1975, Astrophys. J., 196, 83

McKinnon, M. M., \& Stinebring, D. R. 2000, Astrophys. J., 529, 435

Mitra, D., \& Rankin, J. 2017, MNRAS, 468, 4601

Mucciarelli, A., Salaris, M., Lanzoni, B., et al. 2013, ApJL, 772, L27

Nice, D. J., Splaver, E. M., \& Stairs, I. H. 2001, Astrophys. J., 549, 516

Nice, D. J., Taylor, J. H., \& Fruchter, A. S. 1993, Astrophys. J. Lett., 402, L49

Ord, S. M., van Straten, W., Hotan, A. W., \& Bailes, M. 2004, MNRAS, 352, 804

Pavlov, G. G., Kargaltsev, O., Garmire, G. P., \& Wolszczan, A. 2007, ApJ, 664, 1072

Pennucci, T. T. 2015, PhD thesis, University of Virginia ¡EMAIL¿pennucci@email.virginia.eduj/EMAIL;

Press, W. H., Flannery, B. P., Teukolsky, S. A., \& Vetterling, W. T. 1989, Numerical recipes in Pascal. The art of scientific computing

Radhakrishnan, V., \& Cooke, D. J. 1969, ApJ letters, 3, 225 
Rankin, J. M. 1990, Astrophys. J., 352, 247

Ransom, S. M., Ray, P. S., Camilo, F., et al. 2011, ApJL, 727, L16

Ray, P. S., Thorsett, S. E., Jenet, F. A., et al. 1996, Astrophys. J., 470, 1103

Ray, P. S., Abdo, A. A., Parent, D., et al. 2012, ArXiv e-prints, arXiv:1205.3089

Reynolds, M. T., Callanan, P. J., Fruchter, A. S., et al. 2007, MNRAS, 379, 1117

Roberts, M. S. E. 2011, in American Institute of Physics Conference Series, Vol. 1357, American Institute of Physics Conference Series, ed. M. Burgay, N. D’Amico, P. Esposito, A. Pellizzoni, \& A. Possenti, 127-130

Roberts, M. S. E., Mclaughlin, M. A., Gentile, P., et al. 2014, Astronomische Nachrichten, 335, 313

Ruderman, M., Shaham, J., \& Tavani, M. 1989, Astrophys. J., 336, 507

Ruderman, M. A., \& Sutherland, P. G. 1975, Astrophys. J., 196, 51

Sabbi, E., Gratton, R., Ferraro, F. R., et al. 2003, ApJL, 589, L41

Schultz, G. V., \& Wiemer, W. 1975, A\&A, 43, 133

Segelstein, D. J., Rawley, L. A., Stinebring, D. R., Fruchter, A. S., \& Taylor, J. H. 1986, Nature, 322, 714

Shibazaki, N., Murakami, T., Shaham, J., \& Nomoto, K. 1989, Nature, 342, 656

Staelin, D. H., \& Reifenstein, III, E. C. 1968, Science, 162, 1481 
Stairs, I. H., Thorsett, S. E., \& Camilo, F. 1999, Astrophys. J. Suppl. Ser., 123, 627

Stairs, I. H., Faulkner, A. J., Lyne, A. G., et al. 2005, Astrophys. J., 632, 1060

Stappers, B. W., Gaensler, B. M., Kaspi, V. M., van der Klis, M., \& Lewin, W. H. G. 2003, Science, 299, 1372

Szkody, P., Fraser, O., Silvestri, N., et al. 2003, AJ, 126, 1499

The NANOGrav Collaboration, Arzoumanian, Z., Brazier, A., et al. 2015, ApJ, 813, 65

Thorsett, S. E., \& Stinebring, D. R. 1990, ApJ, 361, 644

Thorstensen, J. R., \& Armstrong, E. 2005, AJ, 130, 759

van Kerkwijk, M. H., Breton, R. P., \& Kulkarni, S. R. 2011, ApJ, 728, 95

van Straten, W. 2004, ApJS, 152, 129

-. 2013, ApJS, 204, 13

Verbunt, F., \& Freire, P. C. C. 2014, A\&A, 561, A11

Xu, J., \& Han, J.-L. 2014, Research in Astronomy and Astrophysics, 14, 942

Yan, W. M., Manchester, R. N., van Straten, W., et al. 2011, MNRAS, 414, 2087

Zavlin, V. E., Pavlov, G. G., \& Shibanov, Y. A. 1996, Astron. Astrophys., 315, 141

Zhang, L., \& Cheng, K. S. 2003, Astron. Astrophys., 398, 639 